\title{
Computational Modeling of Fracture in Encapsulation-Based Self-Healing Concrete Using Cohesive Elements
}

\section{DISSERTATION}

Zur Erlangung des akademischen Grades

Doktor-Ingenieur (Dr.-Ing.)

an der Fakultät Bauingenieurwesen

der Bauhaus Universität Weimar

vorgelegt von

Luthfi Muhammad Mauludin

aus

Bandung, Indonesia

(interner Doktorand)

Mentor:

Prof. Dr.-Ing. Timon Rabczuk

Weimar, September 2021 


\title{
Declaration of Authorship
}

\author{
Computational Modeling of Fracture \\ in Encapsulation-Based Self-Healing Concrete \\ Using Cohesive Elements
}

\begin{abstract}
DISSERTATION
for the awarding of the academic degree of Dr.-Ing in the Faculty of Civil Engineering at the Bauhaus-Universität Weimar
\end{abstract}

\author{
Submitted by \\ Luthfi Muhammad Mauludin \\ Born in: March 6, 1977 \\ Sukabumi, Indonesia
}

Assessors:

Prof. Timon Rabczuk

Prof. Roberto Brighenti

Prof. Stéphane Bordas

Date of the defence: September 23, 2021 
I would like to dedicate this dissertation to my loving family, Nunuk Masulah, Hilwa Taffanina, and Haiva Lavie Aydiena for their relentless supports and prays during our stay in Weimar, Germany. Special gratitude for my newly born son, Haitsam Geehaady Mauludin, who gives me invaluable strength and motivation to finish this dissertation. 


\section{Acknowledgements}

I would like to acknowledge RISTEK-DIKTI (Directorate General of Resources for Science, Technology and Higher Education. Ministry of Research, Technology and Higher Education of Indonesia) for their supports and guidance through Overseas Doctorate Degree Scholarship (BPPLN) under funding agreement No: 153.39/E4.4/2014.

My highest appreciation to my supervisor Prof. Dr. -Ing. Timon Rabczuk for his persistent advice and guidance during my dissertation. I also greatly respect for the invaluable cooperation and attention provided by Prof. Xiaoying Zhuang and Md Shahriar Quayum. Their priceless assistances and valuable helps have assisted me in completing this dissertation.

The last but not least, I am obliged to express my gratitude and respect to all my colleagues in ISM (Institute of Structural Mechanics) which are not possible to mention each of them, Digital Bauhaus Lab in Bauhaus-Universität Weimar, Germany (VEGAS Cluster), Rina Sari Tarigan and Erik. Their helps and supports are really appreciated and will be remembered forever. 
Luthfi Muhammad Mauludin

Teknik Sipil, Politeknik Negeri Bandung

(POLBAN), Terusan Gerlong Hilir

Ds. Ciwaruga, Kotak Pos 40012

Bandung, Indonesien

Ehrenwörtliche Erklärung zu meiner Dissertation mit dem Titel:

"Computational Modeling of Fracture in Encapsulation-Based Self-Healing Concrete Using Cohesive Elements"

Sehr geehrte Damen und Herren,

hiermit erkläre ich, dass ich die beigefügte Dissertation selbstständig verfasst und keine anderen als die angegebenen Hilfsmittel genutzt habe. Alle wörtlich oder inhaltlich übernommenen Stellen habe ich als solche gekennzeichnet.

Ich versichere außerdem, dass ich die beigefügte Dissertation nur in diesem und keinem anderen Promotionsverfahren eingereicht habe und, dass diesem Promotionsverfahren keine endgültig gescheiterten Promotionsverfahren vorausgegangen sind.

Bandung, 24. September, 2020

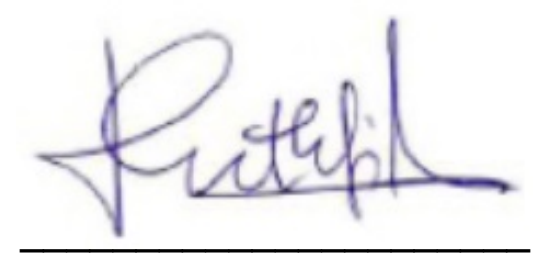




\begin{abstract}
Encapsulation-based self-healing concrete has received a lot of attention nowadays in civil engineering field. These capsules are embedded in the cementitious matrix during concrete mixing. When the cracks appear, the embedded capsules which are placed along the path of incoming crack are fractured and then release of healing agents in the vicinity of damage. The materials of capsules need to be designed in a way that they should be able to break with small deformation, so the internal fluid can be released to seal the crack. This study focuses on computational modeling of fracture in encapsulation-based selfhealing concrete. The numerical model of 2D and 3D with randomly packed aggregates and capsules have been developed to analyze fracture mechanism that plays a significant role in the fracture probability of capsules and consequently the self-healing process. The capsules are assumed to be made of Poly Methyl Methacrylate (PMMA) and the potential cracks are represented by pre-inserted cohesive elements with tension and shear softening laws along the element boundaries of the mortar matrix, aggregates, capsules, and at the interfaces between these phases. The effects of volume fraction, core-wall thickness ratio, and mismatch fracture properties of capsules on the load carrying capacity of self-healing concrete and fracture probability of the capsules are investigated. The output of this study will become valuable tool to assist not only the experimentalists but also the manufacturers in designing an appropriate capsule material for self-healing concrete.
\end{abstract}




\section{Kurzfassung}

Diese Arbeit befasst sich mit der rechnergestützten Modellierung von Brüchen in kapselungsbasiertem selbstheilendem Beton. In selbstheilendem Beton auf Kapselungsbasis sind Mikrokapseln mit Heilmitteln in den Beton eingebettet. Der ankommende Riss sollte erfolgreich auf die Kapsel treffen und die Kapselwand brechen. Die tatsächliche Anwendung von selbstheilendem Beton auf Kapselungsbasis hebt bestimmte ungewisse Schlüsselprobleme hervor, die angegangen werden müssen, wie z. B. die Heilungskapazität, das Design der Kapselmaterialien und die Heterogenität der Mikrostrukturen und ihre Auswirkungen auf den Bruch.

Die Ziele der Studie bestehen aus drei verschiedenen Ebenen. Zunächst soll ein 2D-Frakturmodell auf mesoskaliger Ebene entwickelt werden, das auf unterschiedlichen Dicken und Volumenanteilen von Kapseln basiert. Zweitens, um ein Bruchkriterium zu entwickeln, das auf verschiedenen Kombinationen von elastischen und Brucheigenschaften zwischen der Kapsel und ihrer umgebenden Mörtelmatrix basiert. Drittens, soll das 2D-Modell auf mesoskaliger Ebene in Kombination mit einer statistischen Analyse in ein 3D-Bruchmodell erweitert werden. In diesem komplexen 3D-Modell wird die tatsächliche Heterogenität von Mikrostrukturen innerhalb von Betonmaterial (Aggregate und Kapseln) berücksichtigt und in das numerische Modell aufgenommen.

Die in dieser Studie verwendete Methodik ist die rechnergestützte Modellierung des Bruchverhaltens in auf Kapselung basierendem selbstheilendem Beton unter Verwendung zusammenhängender Elemente. In dieser Studie wurden die verschiedenen Arten numerischer Techniken zur Modellierung von selbstheilendem Beton und ihre Eigen- 
schaften untersucht. Basierend auf dieser umfassenden Übersicht wird der Rahmen neuartiger Berechnungsmethoden identifiziert.

Im nächsten Schritt werden 2D-Bruchmodelle von heterogenem selbstheilendem Beton auf der Mesoskala unter Verwendung von Abaqusund Pyhton-Code entwickelt. Die vorhergesagten Risse werden durch vorab eingefügte kohäsive 4-Knoten- Schnittstellenelemente (CIE) in die anfänglichen Elementnetze dargestellt. Der ABAQUS-explizit mit kleinen Zeitinkrementen wird verwendet, um dieses stark nichtlineare Gleichungssystem zu lösen. Die Auswirkungen des Kapselvolumenanteils, der Caspule-Wandstärke und der Fehlanpassungseigenschaften auf das mechanische Verhalten von selbstheilendem Beton werden untersucht. Das Erweiterungsmodell von 2D nach 3D wird im letzten Schritt ausgeführt. Die Anzahl komplexer 3D-Bruchmodelle wird zufällig generiert und analysiert. Die Validierungen dieses numerischen Modells werden dann mit den jüngsten experimentellen Ergebnissen verglichen.

Die Simulationsergebnisse zeigten, dass die räumliche Verteilung von Aggregaten und Kapseln keinen signifikanten Einfluss auf die globalen mechanischen Eigenschaften von selbstheilendem Beton hat, insbesondere wenn der Volumenanteil der Kapseln im Bereich von 0 bis $2 \%$ liegt. Eine Erhöhung des Volumenanteils der Kapseln von 1.57\% auf $9.42 \%$ verringert die Festigkeit um $6.97 \%$ und erhöht die Dissipationsenergie um 13.66\%. In Bezug auf gebrochene Kapseln zeigte diese Studie, dass eine Erhöhung des Volumenanteils der Kapseln von $1.57 \%$ auf $9.42 \%$ zu einer Erhöhung des Prozentsatzes der gebrochenen Kapseln von $13.5 \%$ auf $92.3 \%$ (Verhältnis 15: 1), $9.8 \%$ auf $72.3 \%$ (Verhältnis 10: 1), $1.5 \%$ bis $16.9 \%$ (Verhältnis $5: 1$ ) und $0 \%$ bis $2.9 \%$ (Verhältnis 1: 1). Zusätzlich tritt die gebrochene Kapsel auf, wenn eine weichere Kapsel (z. B. Ec / Em = 1/7) verwendet wird, verglichen mit der Kapsel mit einer steiferen Kapsel (z. B. Ec / Em $=2$ ). Darüber hinaus erhöht die Erweiterung des Bruchmodells von 2D auf 3D die mittlere Endspannung und die Dissipationsenergie um $27.3 \%$ bzw. $38.6 \%$. 


\section{Contents}

Contents vi

List of Figures $\quad$ ix

Nomenclature xiii

1 Introduction 1

1.1 Background . . . . . . . . . . . . . . . . . . 1

1.2 State of the art .................... 2

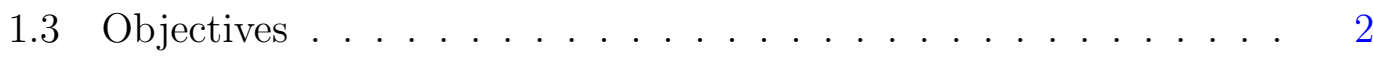

1.4 Methodology ... . . . . . . . . . . . . . 4

1.5 Outline of the dissertation .............. 5

2 Literature Reviews of Concrete Modeling $\quad 7$

2.1 Computational modeling of concrete . . . . . . . . . . . 7

2.1.1 Macroscopic models . . . . . . . . . . . . . . . . . 8

2.1.2 Micromechanical models . . . . . . . . . . . . . 11

2.2 Computational modeling of self-healing concrete . . . . . . . . . . 17

2.2.1 Autogenous healing model . . . . . . . . . . . . . . 18

2.2.2 Autonomous healing model . . . . . . . . . . . 25

3 Numerical Modeling of Encapsulation-Based Self-Healing Concrete

3.1 Microstructures generation at mesoscale level . . . . . . . . . . . . 36

3.1.1 Aggregates and capsules generation . . . . . . . . . . . . 36

3.1 .2 Packing algorithms . . . . . . . . . . . . . . . . . 38 
3.2 Cohesive zone model . . . . . . . . . . . . . . . . . . . 38

3.2.1 Cohesive elements . . . . . . . . . . . . . 38

3.2.2 Traction separation laws . . . . . . . . . . . . 40

3.2.3 Weak form and discretization . . . . . . . . . . . 42

4 2D Numerical Simulations Under Uniaxial Tension 45

4.1 Model parameter . . . . . . . . . . . . . . 45

4.2 Study of mesh size . . . . . . . . . . . . . . . . . . 46

4.3 Numerical results . . . . . . . . . . . . . . . . . . 52

4.3.1 Effect of capsules volume fraction . . . . . . . . . . . 52

4.3.2 Effect of capsule wall thickness . . . . . . . . . . 61

5 The Effect of Mismatch Properties on Fractured Capsule $\quad 67$

5.1 Model parameters . . . . . . . . . . . . . . . 68

5.2 Mesh discretization . . . . . . . . . . . . . . . . . . . . 69

5.3 Parametric studies . . . . . . . . . . . . . . 70

5.4 Numerical results . . . . . . . . . . . . . . . . . 71

5.4.1 Mismatch of elastic and fracture properties . . . . . . . 71

5.4.1.1 Fracture patterns . . . . . . . . . . . . 72

5.4.1.2 Fracture maps . . . . . . . . . . . . 73

5.4.2 Effect of capsule wall thickness . . . . . . . . . . . 77

5.4.2.1 Fracture patterns . . . . . . . . . . . 77

5.4.2.2 Fracture maps . . . . . . . . . . . . . . 79

6 3D Numerical Simulations Under Uniaxial Tension 81

6.1 Microstructures generation at mesoscale level . . . . . . . . . . . . 82

6.1.1 Aggregates and capsules generation . . . . . . . . . 82

6.1.2 Packing algorithms ................ 83

6.2 Cohesive zone model . . . . . . . . . . . . . . . . 85

6.3 Model parameters . . . . . . . . . . . . . . . . . . 87

6.4 Numerical results . . . . . . . . . . . . . . . . . . . . . . . 89

6.4.1 Mesh dependency study . . . . . . . . . . . . . 89

6.4.2 Tensile behavior . . . . . . . . . . . . . . . . . 93

6.4.3 Capsules volume fraction . . . . . . . . . . . . . 96 
6.4.4 Rule of mixtures $(\mathrm{ROM}) \ldots \ldots$. . . . . . . . . 101

6.5 Fracture morphology . . . . . . . . . . . . . . . 102

$\begin{array}{lll}7 & \text { Conclusions } & 107\end{array}$

$\begin{array}{lr}\text { Appendix } & 109\end{array}$

$\begin{array}{lr}\text { References } & 136\end{array}$ 


\section{List of Figures}

2.1 The schematic of particle crack model (right) for the crack (left) [Rabczuk and Belytschko, 2006]. . . . . . . . . . . 9

2.2 Basic concept of microplane model. (a) Idealization of contact planes. (b) Decomposition of strain tensor into microplane. Reproduced from Ožbolt et al. [2001]. . . . . . . . . . . . . . . . . . 11

2.3 The schematic of two-scale methodology for probabilistic-based model of unidirectional fibre-reinforced composite. Reproduced from Trias et al. [2006]. . . . . . . . . . . . . . . . . . .

2.4 Generated 2D random aggregate particles with $46.9 \%$ volume fraction. Grey $=$ mortar matrix, Black = aggregates, White $=$ interfacial zone. (Reproduced from Du et al. [2014]). . . . . . . . . . .

2.5 Schematic of crack propagation criterion based on heterogeneous fracture strength (a) Idealization model. (b) Typical used in the model. (Reproduced from Yang and Xu [2008]). . . . . . . . . . . 16

2.6 Schematic mechanism of further hydration (a) On crack surfaces (b) On cement matrix (UHC represents unhydrated cement particles) (reproduced from Huang and Ye [2012]) . . . . . . . . . . . . 19

2.7 Schematic of computational model ( $\mathrm{InP}=$ inner product, $\mathrm{UHC}$ $=$ unhydrated cement particles) (reproduced from Huang and Ye [2012]) . . . . . . . . . . . . . . . . . 20

2.8 Idealised micro-mechanical model of cementitious materials (reproduced from Davies and Jefferson [2017]) . . . . . . . . . . .

2.9 Microcracks healed in cementitious materials (reproduced from Davies and Jefferson [2017]) . . . . . . . . . . . . . . . 23 
2.10 The diagram of genetic algorithm-artificial neural network (GAANN) model (reproduced from Ramadan Suleiman and Nehdi [2017]) 25

$2.113 \mathrm{D}$ self-healing model with spherical capsules and penny-shaped cracks (reproduced from Lv and Chen [2013]) . . . . . . . . . 27

2.12 3D self-healing model with cylindrical capsules and penny-shaped cracks (reproduced from Lv and Chen [2013]) . . . . . . . . . 27

2.13 2D simplified cell-like crack model pattern (reproduced from Lv et al. [2014]) . . . . . . . . . . . . . . . . . . 28

2.14 3D simplified orthogonal planar crack model pattern (reproduced from Lv et al. [2014]) . . . . . . . . . . . . . . . . . . 28

2.15 Schematic of numerical model-Geometry, boundary conditions and uniaxial loads (reproduced from Gilabert et al. [2015]) . . . . . . . 30

2.16 Detail surfaces between capsule and matrix (reproduced from Gilabert et al. [2015]) . . . . . . . . . . . . . . . . 30

2.17 Diagram of planar crack hitting capsules model (reproduced from Huang and Ye [2016]) . . . . . . . . . . . . . . . .

2.18 Schematic distribution of capsules and the influence zone of planar crack (reproduced from Huang and Ye [2016]) . . . . . . . . . . 31

2.19 PFC3D model under compression loading (reproduced from Zhou et al. [2017]) . . . . . . . . . . . . . . . . . .

2.20 Discrete element model with number of bonds. (a) The initial number of bonds (Ni), (b) The number of bonds after damage $(\mathrm{Nd})$, and (c) The number of bonds after healing $(\mathrm{Nh})$ (reproduced from Zhou et al. [2017]) . . . . . . . . . . . . . . . . .

3.1 Autonomous self-healing using micro-capsules (reproduced from White et al. [2001]) . . . . . . . . . . . . . . . .

3.2 Mesoscale model of encapsulation-based self-healing concrete at consistent volume fraction of aggregates $40 \%$. . . . . . . .

3.3 Inserting cohesive elements in the initial mesh. (a) Initial mesh around a node. (b) Mesh with cohesive elements. (c) 2D 4-noded cohesive elements . . . . . . . . . . . . . . . . . . . . . . 40

3.4 bilinear traction-separation law, reproduced from [Simulia, 2013] . 41 
3.5 Generation of finite element meshes. (a) Initial mesh. (b) Cohesive elements around the sample. (c) Seven sets of cohesive interface elements . . . . . . . . . . . . . . . . . . . 4 44

4.1 Geometry, boundary and loading condition of numerical model . . 46

4.2 Different realization with the same mesh density $(\mathrm{Le}=1 \mathrm{~mm})$ at consistent volume fraction of aggregates $40 \%$ and capsules $1.57 \%$. 47

4.3 Final crack paths for different realization of mesostructures. . . . 49

4.4 Three different meshes with different density. (a) Mesh 1 (Le $=1$ $\mathrm{mm})$. (b) Mesh $2(\mathrm{Le}=0.75 \mathrm{~mm})$. (c) Mesh $3(\mathrm{Le}=0.5 \mathrm{~mm}) . . \quad 50$

4.5 Final crack paths for different mesh density. (a) Mesh 1 (Le $=1$ $\mathrm{mm})$. (b) Mesh $2(\mathrm{Le}=0.75 \mathrm{~mm})$. (c) Mesh $3(\mathrm{Le}=0.5 \mathrm{~mm}) . .51$

4.6 Mean stress-displacement curves for different meshes. . . . . . . . 52

4.7 Stress-displacement curves from 20 random samples with volume fraction of capsules $1.57 \% \ldots \ldots$. . . . . . . . . . 53

4.8 Statistical results of stress-displacement curves from 20 random samples .......................... 53

4.9 Comparison of mean stress-displacement curves in uniaxial tension. 54

4.10 Effects of capsules volume fraction to the peak stress. . . . . . . . 55

4.11 Statistical results of dissipation energy curves from 20 random samples. . . . . . . . . . . . . . . . 56

4.12 Mean energy-displacement curves with different volume fraction of capsules. . . . . . . . . . . . . . . 56

4.13 Effects of capsules volume fraction to the peak dissipation energy. 58

4.14 The relationship between mean stress, mean energy, and volume fraction of capsules. . . . . . . . . . . . . . . . . . 58

4.15 The generation of pre-peak microcracking for different volume fraction of capsules. (a) $\mathrm{Vf}=1.57 \%$ (b) $\mathrm{Vf}=3.14 \%$ (c) $\mathrm{Vf}=6.28 \%$ (d) $\mathrm{Vf}=9.42 \% \ldots \ldots \ldots \ldots \ldots \ldots \ldots$

4.16 Effects of volume fraction of capsules to the percentage of capsules breakage. . . . . . . . . . . . . . . . 60

4.17 Microcapsule samples with different core-wall thickness ratio. (a) Ratio 1:1. (b) Ratio 5:1. (c) Ratio 10:1. (d) Ratio 15:1. . . . . . . 
4.18 Mean stress-displacement curves from volume fraction of capsules $1.57 \%$ with different core-wall thickness ratio. . . . . . . . . . . 64

4.19 The mean of breakage capsules with corresponding core-wall thickness ratio and capsules volume fraction. . . . . . . . . . 65

5.1 Geometry of the model, loading, and boundary conditions . . . . 69

5.2 Mesh discretization of the model. (a) Initial mesh. (b) Inserted cohesive elements around the model. . . . . . . . . . . . . 70

5.3 Failure path of an incoming crack through capsule based on mismatch in elastic and fracture properties. . . . . . . . . . . 73

5.4 Effect of mismatch in elastic properties on fractured capsule. (a) $E_{c} / E_{m}=1$. (b) $E_{c} / E_{m}=1 / 7$. (c) $E_{c} / E_{m}=2 \ldots \ldots$. . . . 75

5.5 Fracture map: Effect of mismatch in fracture properties with different elastic ratios. . . . . . . . . . . . . . . 76

5.6 Capsule samples with different wall thickness. (a) R11. (b) R51. (c) R101. (d) R151. . . . . . . . . . . . . . . . . . . 77

5.7 Failure path of an incoming crack through capsule with different core-wall thickness ratios. . . . . . . . . . . . . . . . . . . 79

5.8 Fracture map: Effect of different capsule wall thickness ratios. . . 80

6.1 Numerically generated 2D mesomodels with different realizations for capsule-based self-healing concrete (volume fraction of capsules $=2 \%) \ldots \ldots \ldots \ldots \ldots \ldots \ldots \ldots \ldots \ldots \ldots \ldots \ldots \ldots \ldots \ldots$

6.2 Numerically generated 3D mesomodels with different realizations for capsule-based self-healing concrete (volume fraction of capsules $=2 \%) \ldots \ldots \ldots \ldots \ldots \ldots \ldots \ldots \ldots \ldots \ldots \ldots \ldots \ldots$

6.3 bilinear traction-separation law for cohesive elements, reproduced from Simulia [2013] . . . . . . . . . . . . . . . 86

6.4 Geometry dimensions, boundary, and loading conditions . . . . . 88

6.5 Different meshes densities used for mesh-dependence study . . . . 90

6.6 Stress-displacement curves from different meshes densities (volume fraction of capsules $=2 \%$ ) . . . . . . . . . . . . 91

6.7 Final crack surfaces from different meshes densities . . . . . . . . 92 
6.8 Stress-displacement curves from different realization microstructures with the same mesh densities (volume fraction of capsules $=$ $2 \%) \ldots \ldots \ldots \ldots \ldots \ldots \ldots$

6.9 Mean stress-displacement curves from statistical analysis of 50 random samples (volume fraction of capsules $=5 \%$ ) . . . . . . . . 94

6.10 Mean dissipation energy curves from statistical analysis of 50 random samples (volume fraction of capsules $=5 \%$ ) . . . . . . 95

6.11 Variation of standard deviation curves of peak stress from 2D and 3D samples (volume fraction of capsules $=5 \%$ ) . . . . . . . . 96

6.12 Numerical samples with different volume fraction of capsules (Vfagg

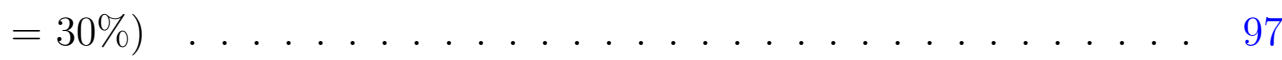

6.13 Effects of capsules volume fraction on the peak stress . . . . . . . 98

6.14 Statistical results of peak stress extracted from 50 random samples 99

6.15 Effects of capsules volume fraction on the fracture dissipation energy 100

6.16 Typical type of fracture evolutions from 3D samples (volume fraction of capsule $=10 \%) \ldots \ldots \ldots \ldots$

6.17 Cutting view of cracking surface evolutions from 3D specimen (volume fraction of capsules $=10 \%) \ldots \ldots$. . . . . . 104

6.18 3D failure model of capsule-based self-healing concrete under uniaxial tension . . . . . . . . . . . . . . . . 105

6.19 Breakage of capsules in tension (only capsules are shown without aggregates and mortar matrix) . . . . . . . . . . . 106 


\section{Chapter 1}

\section{Introduction}

\subsection{Background}

Concrete is the most commonly used materials in construction area due to its high strength and durability relative to its cost. Because of the limited tensile strength, concrete is quite sensitive to the crack formation which can endanger the durability of concrete structures as a whole. The appearance of cracks or microcracks could be occurred on any stages of service life of concrete structures because of excessive loadings, harsh environment exposure, shrinkage, etc. Cracks can lead to the deterioration of concrete structures as aggressive liquids and gasses are able to penetrate into the matrix and hence reduced the mechanical performance of concrete structures. Therefore, the inspection, maintenance and repair works are obviously needed. According to [Cailleux and Pollet, 2009; Van Tittelboom and De Belie, 2013], in Europe, the repair works costs have been estimated to contribute at least around $50 \%$ of the annual total construction budget aside from indirect costs due to the productivity loss. However, when the appearance of cracks or microcracks are not visible and accessible, the repair activities would become difficult and demanding very high costs. Therefore, making self-healing concrete with triggering crack healing mechanism without human intervention in maintenance and increase the service life of structures would be highly beneficial. 


\section{$1.2 \quad$ State of the art}

Over the past decade, a significant amount of effort has been made towards the development of new concrete materials where the damage can be detected and healed automatically by the material itself at micro or even nanoscale. Recent intense research interests on self-healing concrete were raised after [White et al., 2001] published their work on micro-capsules based epoxy-polymers in Nature in 2001. Apart from micro-capsules healing, other healing methods such as calcite bacterial healing or mineral fiber have also shown potentials in concrete. [Zemskov et al., 2011] for instance developed a statistical model to determine the probability of cracks hitting spherical capsules. [Lv et al., 2014] investigated the dosage required for the long capsule in cementitious matrix using combined geometrical probability theory with binomial probability distribution. The probability of cracks hitting capsules with particular volume fraction in $2 \mathrm{D}$ and $3 \mathrm{D}$ model was also developed by [Lv and Chen, 2013]. The possibility of using different capsules thickness and the interaction between capsule and the matrix was studied by [Gilabert et al., 2015]. Those aforementioned studies provided useful insight on the probability of cracks hitting the micro-capsules but they did not take into account for the physical cracking process such as the mechanical properties of the matrix material and micro-capsules. In the area of computational model, preliminary review shows that past studies of the self-healing concrete model are primarily focused on continuum models which concrete is considered as homogenized material [Barbero et al., 2005; Darabi et al., 2012]. It means that the actual heterogeneity of microstructures in concrete material have been neglected and excluded in the numerical model.

\subsection{Objectives}

Recently self-healing concrete has received a tremendous attention from researchers in civil engineering sciences due to their wide range of capabilities, especially in micro-capsule-based self-healing concrete. Micro-capsule contained healing agents are embedded in the concrete. The incoming crack should break the capsule shell to release the liquid and healing process in the damage area will be occurred. De- 
spite the recent breakthrough in self-healing techniques, the actual application of self-healing concrete structure highlights on particular uncertain key issues that need to be addressed:

1. The healing capacity, i.e. will the appearance of crack successfully hit the capsule? So the healing liquid can completely fill and repair the crack. Recently, experimental work proved that the interfacial bonding between the capsule wall and the mortar matrix is not perfect [10];

2. The volume fraction of capsules and its global response to the mechanical properties of concrete. It still remains unclear about the effects from volume fraction of capsules on load carrying capacity of the original concrete;

3. The core-wall thickness ratio of capsule and its global response on load carrying capacity of the original concrete and fracture probability of capsules;

4. The mismatch properties (elastic and strength properties) between the capsule and its surrounding mortar matrix and their effects on fractured capsule.

The novelty of the research is to develop fracture model for the design of the next generation encapsulation-based self-healing concrete materials. It should accommodate for the design of new healing capsules as well as their interaction with the neighboring particles inside the concrete. To reach this objective, the research focused at four different levels (specific objectives):

1. Comprehensive reviews of recent developments in computational modeling techniques in self-healing concrete;

2. Developing a 2D fracture model at mesoscale level based on different thickness and volume fraction of capsules;

3. Developing a fractured capsule maps based on different combinations of elastic and fracture properties between capsule and its surrounding mortar matrix; 
4. Extending the 2D model into 3D fracture model at mesoscale level combined with statistical analysis. In the context of this 3D model, the actual heterogeneity of microstructures inside concrete material (aggregates and capsules) is considered and included in the numerical model.

The output of this research will become an asset to the engineering and material sciences, industrial practitioners as well as the particular manufacturers company in developing an appropriate capsule material for encapsulation-based self-healing concrete. Furthermore, results from the present project are in line with the necessity of sustainable development of smart building and infrastructures materials.

\subsection{Methodology}

The primary research focus is computational modeling of fracture in encapsulationbased self-healing concrete. The methodology of the research is carried out as follows:

1. The random $2 \mathrm{D}$ models of heterogeneous self-healing concrete at mesoscale level are developed using a cohesive zone model in Abaqus and Python code. Some key activities in this stage are:

- Aggregates distribution. The Fuller curve is used to determine distribution of aggregates inside the mortar matrix. The sieve size of 2.36 $\mathrm{mm}$ is taken as the cut-off size of coarse aggregates.

- Capsules generation. The capsules are assumed to be made of Poly Methyl Methacrylate (PMMA). The model used $2.0 \mathrm{~mm}$ as diameter of the capsule and its thickness is considered as diameter $/ 27$.

- Cohesive elements insertion. The predicted cracks are represented by pre-inserted 4-node cohesive interface elements (CIE) into the initial element meshes (mortar matrix, aggregates, capsules, and their interfaces).

- Loading generation. The uni-axial tension loading is applied in this study. 
2. The extensive 2D simulations of fracture behavior from capsule using different combinations of elastic and fracture properties between capsule and its surrounding mortar matrix are carried out.

3. The fracture model is extended into 3D heterogeneous model. The numbers of complex 3D samples are randomly generated and analyzed. The results of these numerical models are then compared to the recent experimental works.

\subsection{Outline of the dissertation}

This dissertation consists of seven chapters including an introduction part. The introduction section gives comprehensive summary to the subject explained in this research. A brief overview has been presented in this chapter in relation to the computational modeling of fracture in encapsulation-based self-healing concrete. A discussion has been made on how importance numerical model of fracture in encapsulation-based self-healing concrete considering the heterogeneity of microstructures embedded in the mortar matrix is included in the model.

Chapter 2 provides study of the literature based on the bibliographical researches. The recent developments of self-healing concrete are reviewed. The prospective materials and its healing mechanism applied in concrete structures are given, and the numerical modeling approach in the field of self-healing concrete is discussed. Chapter 3 explains the numerical modeling strategy in encapsulation-based selfhealing concrete with respect to packing algorithms, aggregates and capsules distributions, cohesive zone model, and loading condition. Cohesive element and its traction-separation laws are also presented.

Chapter 4 deals with the 2D numerical simulations of encapsulation-based selfhealing concrete under uni-axial tension. Effects of capsules volume fraction and capsule-core wall thickness ratio on load carrying capacity and fracture pattern are investigated. The output results such as stress-displacement relationships, energy-displacement curves, and crack propagation are also presented and discussed.

Chapter 5 presents the effect of mismatch properties from capsule materials on 
fracture probability. The mismatch for both elastic and fracture properties in terms of properties ratio between capsule materials and its surrounding mortar matrix are investigated and plotted on the fracture maps.

Chapter 6 deals with the 3D numerical simulations of encapsulation-based selfhealing concrete under uni-axial tension. Effects of capsules volume fraction on load carrying capacity and fracture energy are studied. The heterogeneity of microstructures used in the model and its effect on fracture morphology are observed.

Chapter 7 provides the major conclusions of the whole simulations used in the study. The reliability and the limitation of the models are also discussed together with the suggestion for the future researches. 


\section{Chapter 2}

\section{Literature Reviews of Concrete Modeling}

\subsection{Computational modeling of concrete}

The complex behavior of quasi-brittle material, such as concrete, has been used in many engineering structures due to its high strength and durability. The mechanical behavior of concrete is determined by its heterogeneity due to the presence microcracks, voids, aggregates, etc.

Over the past two decades, both novel and efficient computational techniques for fracture have been developed by researchers including cohesive zone model [Hillerborg et al., 1976], the extended finite element [Moës and Belytschko, 2002], meshfree methods [Amiri et al., 2014], cracking particles methods [Rabczuk and Belytschko, 2004; Rabczuk et al., 2010d], efficient remeshing techniques [Areias et al., 2013, 2014], phase field methods [Areias et al., 2016b], screened Poisson models [Areias et al., 2016a], peridynamics [Silling and Lehoucq, 2010], dualhorizon peridynamics [Ren et al., 2016], the extended particle difference method [Yoon and Song, 2014a,b,c], and the multiscale modeling of material failure [Belytschko and Song, 2010; Budarapu et al., 2014a,b; Song and Yoon, 2014; Talebi et al., 2014, 2015] to name a few.

Modeling fracture process in concrete material is not an easy task. Even though there are a lot of researches have been made to explain this complex process, but 


\section{Literature Reviews of Concrete Modeling}

until now there is no exact model able to simulate all nature's aspects of concrete fracture and describe it in detail. In general, Computational modeling of concrete fracture is divided into two categories, such as macroscopic and micromechanical models. The macroscopic models are based on phenomenological approach which are derived from theory plasticity and fracture mechanics, whereas micromechanical models are constructed by determining interaction between microstructures inside concrete material and its behavior in macroscopic scale [Djoković et al., 2013a,b].

\subsubsection{Macroscopic models}

There are at least two different techniques available in macroscopic models, such as discrete and continuum approaches. In the discrete method, the displacement field discontinuities obtained from fracture process are directly introduced into the numerical system. It is based on fracture mechanics theory and more sufficient to handle localization of the damage [Jendele et al., 2001]. In the prior approach, usually the crack is modeled to be within an element as a 'fictitious' crack and smeared crack models [Hillerborg et al., 1976; Jirásek and Zimmermann, 1998]. As an alternative to the smeared crack model, the discrete approach is introduced with a discontinuity. The application of discrete crack into the model can be carried out with element separation method along the boundaries $[\mathrm{Xu}$ and Needleman, 1996] or propagate arbitrary within an element without remeshing [Belytschko et al., 1994b; Samaniego Alvarado, 2003; Wells and Sluys, 2001]. Rabczuk et al. [2005] developed a fictitious/smeared crack model for fracture in reinforced concrete structure. They combined fixed with rotating crack to simulate cracking process in the concrete. The combined fixed-rotating crack model will guarantee the deformation of material in arbitrary directions.

The applicability of particle methods with Lagrange multipliers in modeling of static fracture in reinforced concrete structures was introduced by Rabczuk and Belytschko [Rabczuk and Belytschko, 2006]. They used a cohesive crack particle method to simulate fracture process in the concrete which was proposed in dynamic case [Rabczuk and Belytschko, 2004]. The geometry of cracks is determined by a set of restricted discrete cracks which lie on the particular particles as 


\section{Literature Reviews of Concrete Modeling}

shown in Fig. 2.1. With this technique, the direction of crack path is always pass through particles since geometry of the crack is not provided. In the continuum-

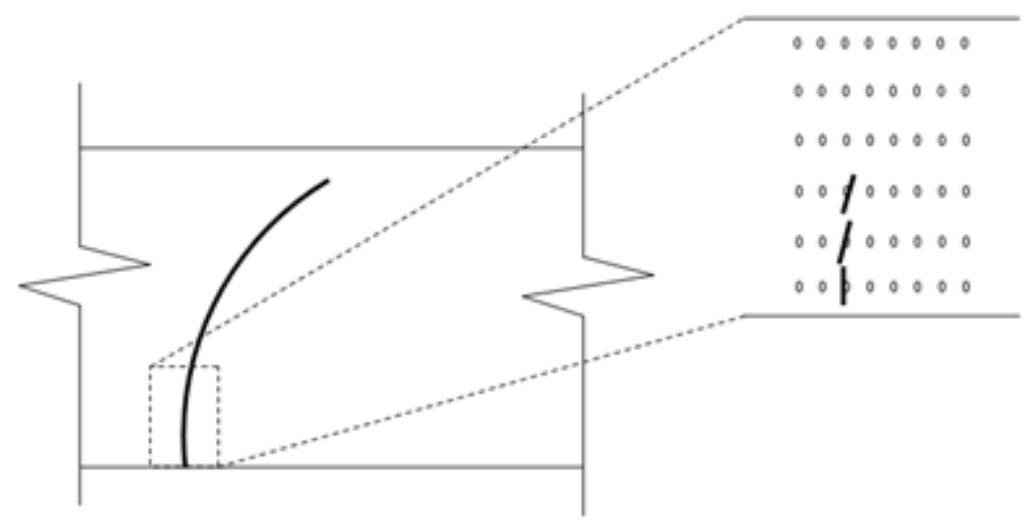

Figure 2.1: The schematic of particle crack model (right) for the crack (left) [Rabczuk and Belytschko, 2006].

based technique, the stress-strain relationship is defined at the macroscopic scale. Theoretically speaking, it is possible to define constitutive relationship between stress and strain at this scale to determine macroscopic behavior of the material. Fracture mechanism in this approach must be considered as dissipative process in the material level which treat cracks as micro-cracks and diffused into the whole representative elementary volume of the material.

There are so many commonly used nonlinear constitutive model for concrete found in the literature, such as plasticity, damage mechanics, and combination between them. Stress-based plasticity approach are usually applied to characterize behavior of concrete under triaxial stress, since the yield surface of concrete at particular hardening region corresponds to the concrete strength [Červenka and Papanikolaou, 2008; Folino and Etse, 2012; Grassl et al., 2002; Menetrey and Willam, 1995; Papanikolaou and Kappos, 2007]. To represent gradual reduction of the unloading stiffness which are detected in the experiments, strain-based isotropic damage mechanics is introduced [Carol et al., 2001; Ortiz, 1985; Tao and Phillips, 2005; Voyiadjis and Kattan, 2009]. Combination of plasticity and isotropic damage to explain special phenomenon that is existed in the experiment, such as irreversible deformation, has been commonly used by some researchers 


\section{Literature Reviews of Concrete Modeling}

[Grassl, 2008; Grassl and Jirásek, 2006; Hofstetter et al., 2013; Jason et al., 2006; Nguyen and Houlsby, 2008; Nguyen and Korsunsky, 2008; Sánchez et al., 2012; Voyiadjis et al., 2008]. This model can be used to simulate both of tensile and compressive behavior of concrete and not limited to the low confined compression stress. Oliver et al. [2004] introduced continuum strong discontinuity approach (CSDA) into the fracture model of concrete. To avoid instabilities caused by multiple propagating cracks interaction, they also introduced a viscous perturbation on the crack surface.

Microplane technique developed by Bažant and coworkers [Bažant and Gambarova, 1984; Bazant and Oh, 1983; Bažant et al., 1996] provides an alternative approach to simulate inelastic modeling of concrete behavior. The relationship between stress and strain tensor in the material is determined by various planes of orientations which are indicated as damage planes in micro scale and plays a role as contact surface between aggregate particles in concrete material as illustrated in Fig. 2.2.

Ožbolt et al. [2001] introduced relaxed kinematic constraint into microplane model. Each plane in microplane consists of normal strain $\left(\varepsilon_{N}\right)$ and shear strain $\left(\varepsilon_{T}\right)$ components. The normal component is split into volumetric and deviatoric parts $\left(\varepsilon_{V}, \varepsilon_{D}\right)$ whereas shear strain into perpendicular components $\left(\varepsilon_{M}, \varepsilon_{K}\right)$ as given by

$$
\begin{gathered}
\vec{\varepsilon}_{N}=\left(\varepsilon_{D}+\varepsilon_{V}\right) \\
\vec{\varepsilon}_{T}=\varepsilon_{M} \vec{m}+\varepsilon_{K} \vec{k}
\end{gathered}
$$

They introduced discontinuity function, $\psi$ into eq.2.1 and 2.2, except for volumetric part, as kinematic constraint to model discrete tensile cracking. This discontinuity function value $(0 \leq \psi \leq 1)$ is defined by volumetric stress-strain relationship to indicate the discontinuity in each individual microplane and combined with macroscopic strain tensor, $\varepsilon_{i j}$ to determine the effective microplane strains as

$$
\varepsilon_{V}=\frac{\varepsilon_{k k}}{3}, \quad \varepsilon_{D}=\left(n_{i} n_{j} \varepsilon_{i j}-\varepsilon_{V}\right) \psi \quad \text { (normal components) }
$$




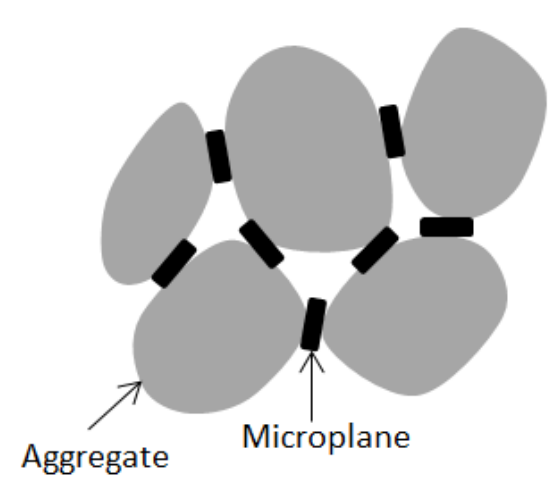

(a)

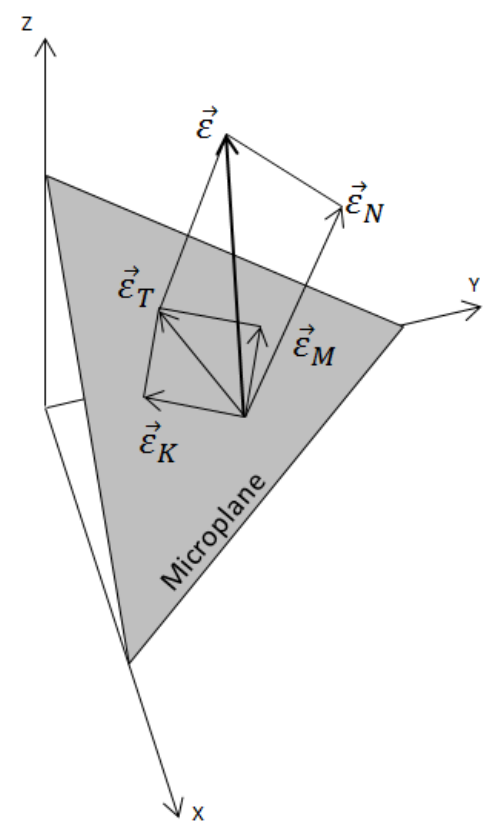

(b)

Figure 2.2: Basic concept of microplane model. (a) Idealization of contact planes. (b) Decomposition of strain tensor into microplane. Reproduced from Ožbolt et al. [2001].

and

$$
\varepsilon_{M}=m_{i} n_{j} \varepsilon_{i j} \psi, \quad \varepsilon_{K}=k_{i} n_{j} \varepsilon_{i j} \psi . \quad \text { (shear components) }
$$

\subsubsection{Micromechanical models}

In contrast with the aforementioned models, the micromechanical approach treats the heterogeneity of concrete microstructures as different phases to provide accurate characteristic of fracture behavior. At the mesoscale, concrete is represented by at least three phases materials, such as coarse aggregate, mortar matrix, and the interface between these phases. In order to characterize the heterogeneity in concrete materials, there are two basic approaches: the direct approach and the indirect approach [Su et al., 2010; Yang et al., 2009] which will be discussed in the following sections.

The main idea of the direct approach is all microstructures in a concrete material 


\section{Literature Reviews of Concrete Modeling}

such as mortar matrix, aggregate, and the interfaces between them are precisely modeled by finite elements. The material properties of each microstructures can be assigned directly afterward into the particular elements. Some researchers had been proposed direct approach to explicitly modeled the mortar matrix, coarse aggregates with random size and shape, and the interfaces between them in 2D model under tension and compression loading [López et al., 2008a,b; Teng et al., 2004; Zhu et al., 2015]. The probability of cracking inside matrix has been investigated by Trias et al. [2006]. They developed two scale methodology of cracking matrix formation perpendicular to the cross section of fibres in unidirectional fibre-reinforced composite materials. The statistical representative volume element (SRVE) combined with two scale methodology is applied to capture transformation from microscale to macroscale. At microscale, the position of fibres are considered to be random, whereas the elastic and failure properties are assumed to be constant. The material is considered to be homogeneous at the macroscale, only the failure properties is assumed to be random. The schematic of this methodology can be seen in Fig. 2.3

The numerical modeling of concrete failure behavior at mesoscale level was conducted by Du et al. [2014]. The concrete material was considered as three phases consist of mortar matrix, aggregate, and the interfacial zone between them. They used circular shape as aggregate particles and thin layers role as interfacial transition zone between mortar matrix and aggregates. They determined the distribution size of the aggregates to follow the Fuller's curve and then placed randomly into the mortar matrix one by one using the well known "take-and-place" technique [Grassl and Jirásek, 2010; Zemskov et al., 2014; Zhou and Hao, 2008] without overlapping between particles occurred. They generated 2D mesoscale concrete with volume fraction of aggregate is $46.9 \%$. The dimension of specimen is $150 \mathrm{mmx} 150 \mathrm{~mm}$ which includes six pieces of medium aggregate with diameter $30 \mathrm{~mm}$ and 56 pieces of the small one with diameter $12 \mathrm{~mm}$. The schematic of generated random specimen can be seen in Fig. 2.4

Monte carlo simulation of concrete fracture at mesoscale level was investigated by Wang et al. [2015a,b, 2016]. Combination analysis between numerical and statistical approach of heterogeneous concrete at mesoscale was conducted by Wang and Jivkov [2015]. They developed both of mesoscale heterogeneous con- 


\section{Literature Reviews of Concrete Modeling}

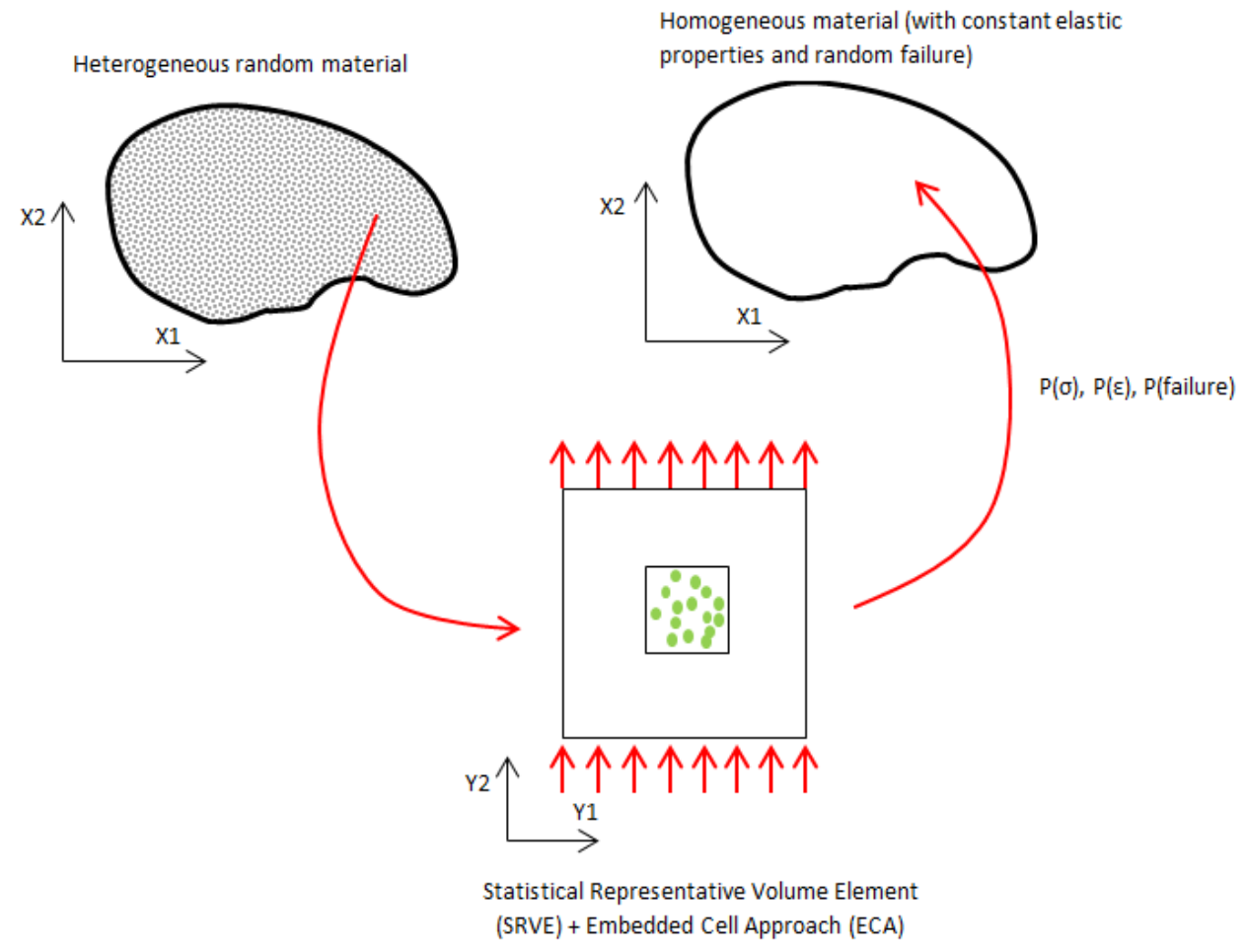

Figure 2.3: The schematic of two-scale methodology for probabilistic-based model of unidirectional fibre-reinforced composite. Reproduced from Trias et al. [2006].

crete with random elliptical aggregates and circular voids (2D) and ellipsoidal aggregates and spherical voids (3D). The statistical evaluation are employed to the all outputs started with standard deviation $s$ from $n$ samples as given by

$$
s^{2}=\frac{1}{n-1} \sum_{i=1}^{n}\left(x_{i}-\bar{x}\right)^{2}
$$

where $\bar{x}=(1 / n) \sum_{i=1}^{n} x_{i}$ is the average result from a series of samples and $x_{i}$ is the result of sample $i$. Later on, the coefficient of variation is used to differentiate the output from different number of samples as following:

$$
\varepsilon=\frac{s}{\bar{x}}
$$

The equation 2.6 defines variation of measured output relative to its mean value. In the cohesive zone model, for establishing displacement continuity across the 


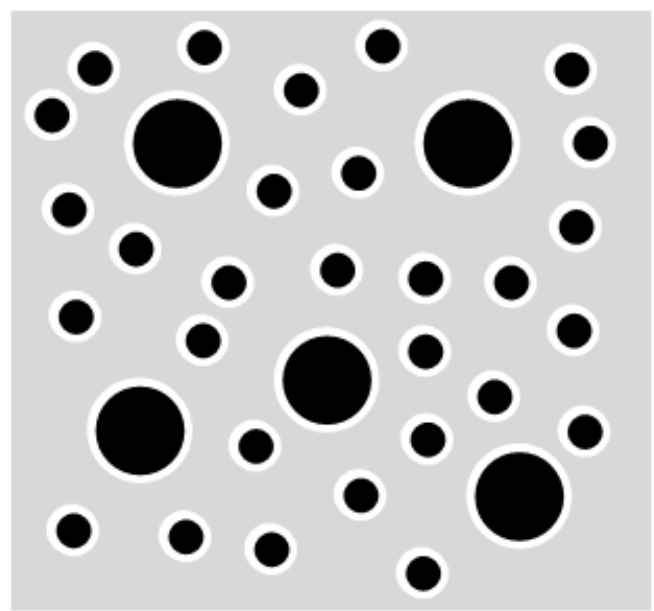

Figure 2.4: Generated 2D random aggregate particles with $46.9 \%$ volume fraction. Grey $=$ mortar matrix, Black $=$ aggregates, White $=$ interfacial zone. (Reproduced from Du et al. [2014]).

interface, the initial stiffness of tension is critical to choose before the tensile strength is achieved. They used the following criterion to predict initial stiffness:

$$
k_{n 0}=k_{s 0}=k_{t 0}=\frac{c(1-\nu)}{b(1+\nu)(1-2 \nu)} E
$$

where $E$ and $\nu$ are Young's modulus and Poisson's ratio, respectively, $b$ is the characteristic length, and $c$ is dimensionless value ranging between 10-100.

In contrary to the direct approach, the indirect approach considered the different phases of concrete microstructures in implicit way. Variation of spatial random field with particular correlation is often used to assign specific material properties in the domain of interest such as tensile strength and fracture energy. Many new techniques have been introduced by some researchers to generate some random fields of material properties [Graham-Brady and Xu, 2008; Koutsourelakis and Deodatis, 2006; Xu and Graham-Brady, 2005], but most of those approaches have not been applied into fracture modeling. Application of Weibull random fields into material properties to study propagation of the cracks in concrete beams were investigated by Bruggi et al. [2008]; Most [2005]; Yang and Xu [2008]. Heterogeneous cohesive (HC) crack model to predict macroscopic behavior based on Weibull random fields of fracture properties were developed by Yang and 


\section{Literature Reviews of Concrete Modeling}

$\mathrm{Xu}$ [2008]. They used new stress-based criterion to define direction in which a crack propagates. Since the fracture strength is spatially random generated, it forced the propagation of crack to the direction where the fracture strength is low because of weak interfacial zone or voids as can be seen in Fig. 2.5. The current crack tip and the incremental length $\Delta a$ forms an angle $\alpha_{0}$ with the global axis $\mathrm{X}$. The angle between the current and next crack tip should be less than $90^{\circ}$. The upcoming crack will be placed at radius $\Delta a$ from half circle to the centered of current tip as illustrated in Fig. 2.5(a). Since the heterogeneity of fracture properties is main focus, they introduced a tendency indicator as direction function from crack propagation $\alpha$

$$
C\left(\Delta_{a}, \alpha, \omega\right)=\frac{S(\alpha, \omega)}{f_{t}(X, \omega)}=\frac{S(\alpha, \omega)}{f_{t}\left(\Delta_{a}, \alpha, \omega\right)}
$$

where $S(\alpha)$ is the normal stress exist on $\alpha$ plane for the current crack tip and only positive value which will be considered. The equation 2.8 defines tendency $\alpha$ direction at which the crack will propagate. The crack will tend to propagate into the point with lowest $f_{t}$. In other words, with this model, the crack will propagate into the direction which has the highest tendency indicator defined by

$$
\frac{\partial C(\alpha, \omega)}{\partial \alpha}=0 \quad \text { and } \quad \frac{\partial^{2} C(\alpha, \omega)}{\partial \alpha^{2}} \leq 0
$$

with

$$
C(\alpha, \omega)>0 \quad \text { and } \quad \alpha_{0}-90^{\circ} \leq \alpha \leq \alpha_{0}+90^{\circ} .
$$

The equation 2.9 can be solved by computing $C$ in every $1^{\circ}$ which leads to the maximum $C$ as the direction of crack propagation. The next tip of the crack is always existed in the neighboring edges of current tip as described by blue dashed straight lines where the usual mesh is exist before remeshing (see Fig. 2.5(b)). Grassl et al. [2012] developed mesoscale concrete fracture modeling with focused on the size and boundary conditions. They considered only large aggregates embedded in meso-structure and separated by interfacial zone. The discrete lattice approach is applied to simulate the mechanical response from those three phases. The spatial distribution of dissipated energy randomly applied in the mesoscale analysis to predict fracture process zone of concrete materials. 


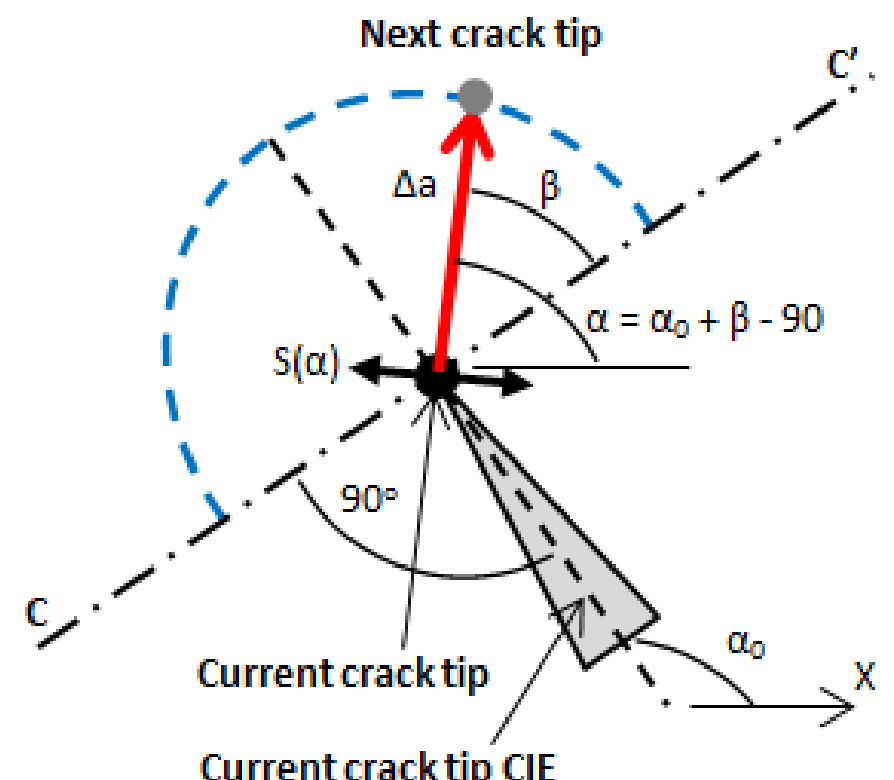

(a)

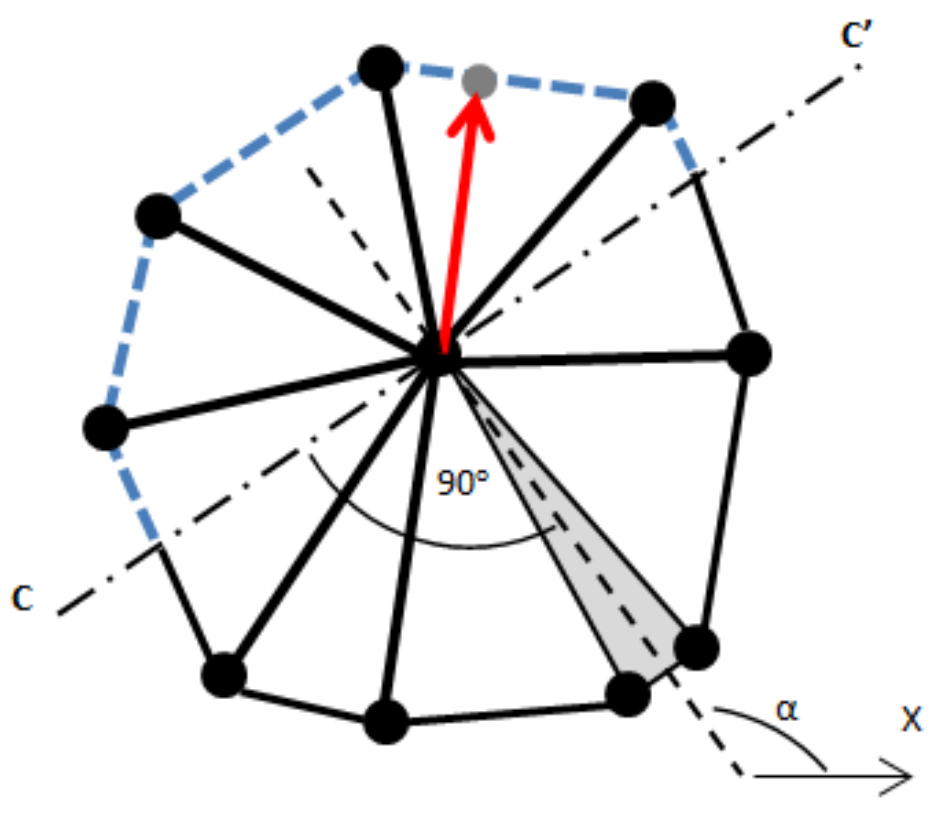

(b)

Figure 2.5: Schematic of crack propagation criterion based on heterogeneous fracture strength (a) Idealization model. (b) Typical used in the model. (Reproduced from Yang and $\mathrm{Xu}[2008])$. 


\subsection{Computational modeling of self-healing con- crete}

The appearance of the microcracks can lead to the severe damage and causing to the strength degradation on any stages of concretes service life. However, when the appearance of these cracks are not accessible and visible, repair activities would not become easy and require very high costs. In this case, applying selfhealing concrete with particular healing mechanism would be very beneficial.

The development of self-healing concrete has attracted a lot of attention due to its inherent ability of automatic crack detection and crack repairing with the goal of significantly reducing the costs for infrastructure maintenance. Different strategies of self-healing mechanism in cementitious materials were reviewed by Wu et al. [2012]. They covered different possible mechanism of self-healing based on substantial experimental and practical works. The comparison between different healing techniques and healing agents are also discussed. The capability of self-healing in Engineered Cementitious Composite (ECC) is also introduced as summary of their review. Van Tittelboom and De Belie [2013] conducted comprehensive review of self-healing approaches in cementitious materials. They discussed the advantages and disadvantages from different types of healing agents and encapsulation techniques. The different mechanism that can trigger to activate self-healing was also evaluated. The review of self-healing concrete based on the three key taxonomies, i.e. natural self-healing, chemical self-healing and biological self-healing were presented by Talaiekhozan et al. [2014]. They reviewed the unexpected cracking of concrete with focusing on biological processes. Lv and Chen [2014] discussed self-healing technologies both of autogenous and autonomous types. They reviewed the improvement of durability and performance of self-healing structures based on experimental and practical investigation. Brief overview of analytical and simulation model of self-healing in cementitious materials were also presented. The effectiveness of self-healing with respect to the application in actual structures was evaluated by Ahn et al. [2017]. They reviewed all researches on ultrasonic-based nondestructive methods and case studies relating to the self-healing concrete. Both the applicability and limitation of different nondestructive methods in assessing self-healing performance were also 


\section{Literature Reviews of Concrete Modeling}

highlighted.

In general, existing healing methods in cementitious materials can be classified into two categories: firstly intrinsic healing where an autogeneous healing is accomplished by the cement composition itself, and secondly extrinsic (autonomous) healing that requires additives to react with cement. The microcapsules based self-healing method, which is claimed among the most promising techniques, belongs to the latter.

\subsubsection{Autogenous healing model}

The autogenous healing is one of the classic type of self-healing in concrete. The healing mechanism attributed to the microcracks is due to re-hydration of unhydrated cement particles in concrete matrix [Hearn, 1998]. Some researchers have investigated and concluded that the main contributions of self-healing in cement-based materials were further hydration of unhydrated cement particles and the nucleation of calcite [Ahn and Kishi, 2010; Edvardsen, 1999; He et al., 2011; Schlangen et al., 2006]. In terms of numerical model, recently, some of researchers have developed a novel computational model to investigate the behavior of autogenous healing in self-healing concrete. Lv and Chen [2012] investigated the efficiency of self-healing with hydration reaction of unhydrated cement nuclei. They used splitting crack and dome-like crack modes to model the cracking in the unhydrated nuclei. The self-healing efficiency was presented in term of geometrical probability. They showed that the degree of self-healing efficiency is characterized based on the crack modes. Moreover, Huang and Ye [2012] proposed model of self-healing by providing extra water on unhydrated cement for further hydration. They assumed there exist amount of water stored in capsules and can be passed by cracks and unhydrated cement particles as well. These unhydrated particles were exposed on both of crack surfaces and embedded inside cement paste as shown in Fig. ??. HYMOSTRUC3D was used to simulate the distribution of unhydrated cement particles. The dimension of a micro-crack with the size of $40 \mathrm{~mm}$ (length) x $40 \mathrm{~mm}$ (depth) x $10 \mu \mathrm{m}$ (width) was assumed to pass through a water capsule due to hydration formed in the cement paste. The ion diffusion model based on Ficks second law was used to calculate the concen- 

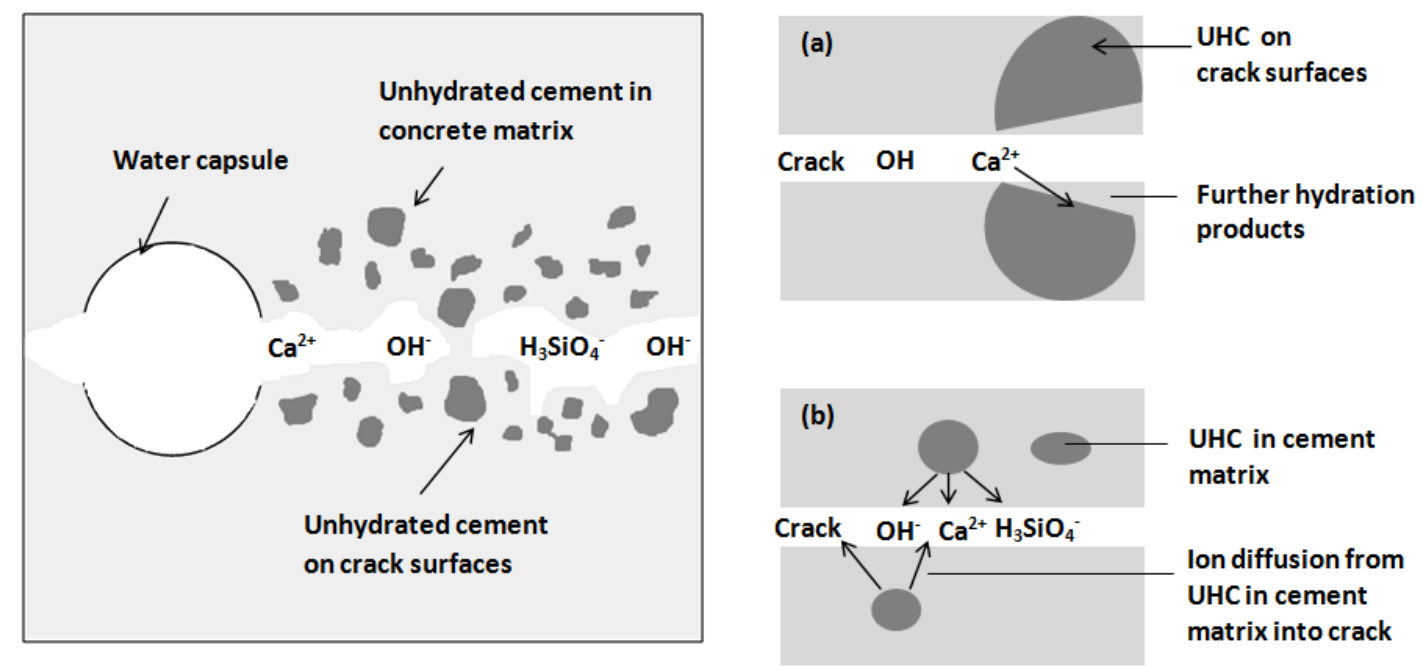

Figure 2.6: Schematic mechanism of further hydration (a) On crack surfaces (b) On cement matrix (UHC represents unhydrated cement particles) (reproduced from Huang and Ye [2012])

tration of ions in each micro pixel. A water-filled section with the size of $100 \mu \mathrm{m}$ x $100 \mu \mathrm{m}$ was modelled to simulate further hydration processes using thermodynamics model. The schematic of simulation model is illustrated in Fig. 2.7. The result of their simulations concluded that the amount of unhydrated cement and extra water on further hydration process can be optimized quantitatively based on self-healing efficiency. Numerical modeling of self-healing capacity in cementbased materials was conducted by Di Luzio et al. [2014]; Ferrara et al. [2016] using micro-plane model M4 and the solidification-micro-prestress theory. This model based on hygro-thermal-chemical problem which is able to capture all the major effects of concrete behavior such as creep, shrinkage, cracking, etc. They incorporated self-healing effects into some internal variables in the model which characterize the process, diffusivity and the repairing effects. The good agreement between experimental and numerical results were shown for all simulated experimental cases. They also showed that the mix composition and duration of exposure are significantly influence to the recovery of load carrying capacity. Hilloulin et al. [2014] investigated self-healing phenomenon on Ultra High Performance Concrete (UHPC). The autogenous healing process by further hydration 


\section{Literature Reviews of Concrete Modeling}

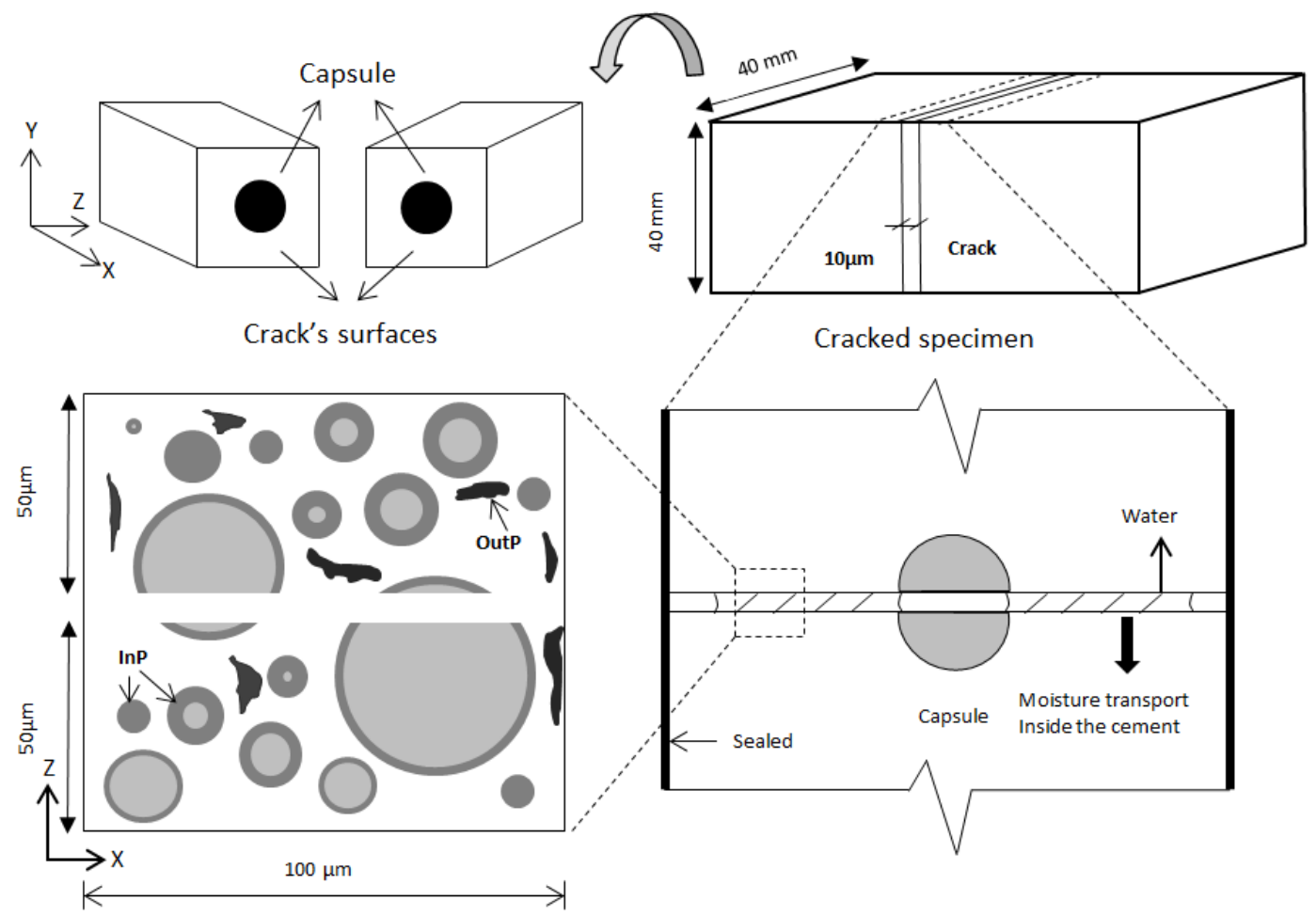

Figure 2.7: Schematic of computational model $(\mathrm{InP}=$ inner product, $\mathrm{UHC}=$ unhydrated cement particles) (reproduced from Huang and Ye [2012])

in the material was simulated using hydro-chemo-mechanical model which has been implemented in the finite element code Cast3M [Hilloulin et al., 2016]. This model calculated the self-healing potential by compare the mechanical properties between virgin and healed model. The simulation results revealed that the predicted corresponding load to the end of the first slope are overestimated. This phenomenon is due to the discontinuity of mechanical properties around twophases modes. The assesment of cracking and healing in changing modal parameters of concrete beams has been evaluated by Savija and Schlangen [2015]. They used natural frequencies changes to identify the damage and therefore the indication of healing could also be taken from the recovery of natural frequencies. Dynamic behavior of concrete beams was modelled using spectral element method (SEM) combined with Delft lattice model to investigate the effects of cracking and healing on modal properties of concrete beams. The 2D simulation showed 


\section{Literature Reviews of Concrete Modeling}

that the appearing microcracks prior to the peak load caused a small decrease in the first natural frequency. The natural frequency of healing events are recovered (except for the first one), but it still lower than the first one.

The capability of cohesive zone model to simulate self-healing materials was investigated by Abu Al-Rub and Alsheghri [2015]. They developed cohesive zone damage-healing model (CZDHM) based on thermodynamics law. The healing mechanism is thermally stimulated through molecular interdiffusion. Damage and healing was described by the damage/healed fraction of the cohesive bonds and its value ranging from 0 to 1 . The internal degradation variable $D$ and $h$ are dimensionless such that $D=0$ means no damage occurred and $D=1$ indicates complete damage, whereas $h=0$ means no healing occurred and $h=1$ indicates complete healing of the damage. They demonstrated the healing model of mode I fracture using single 2D cohesive element COH2D4 with size $1 \mathrm{~mm} \times 1 \mathrm{~mm}$. The results showed that increasing temperature and resting time will eventually increase the healing variable of materials.

Aliko-Benítez et al. [2015] developed numerically an uncoupled healing model for concrete based materials with incorporating physicochemical approach. They proposed healing mechanism which activated through precipitation of calcium carbonate inside the cracks. The mechanical variables was extended simultaneously both of damage and healing process. Their preliminary models revealed that using different model parameters have significantly influence not only on the results but also the qualitative trend derived from the experimental results. Hazelwood et al. [2015] presented numerical model to compare long-term behavior of a new self-healing concrete system (LatConX) with the standard reinforced concrete. They composed the model from a set of sub-models which combined transient thermo-mechanical behavior. The self-healing was incorporated to the mortar/concrete constitutive model in the form either due to the healing additive or autogenous healing which could determine the degree of healing. They assumed healing process was instantaneously occurred in term of healing time and its degree instead of following the healing function. The couples model of a layered beam and thermo-mechanical transient has been validated with experimental data. The results showed that LatConX is succeed to reduce $65 \%$ of shrinkage stress and half of the damage is healed. 


\section{Literature Reviews of Concrete Modeling}

The effects of healing due to ongoing hydration on mechanical recovery were investigated by Hilloulin et al. [2016] both of experimental and numerical. They conducted experimental works on cracked specimens at early stage to investigate some various parameters such as age at cracking, initial crack width and healing time. The autogenous healing mathematical model of an early-age cement-based materials was proposed by Chitez and Jefferson [2016]. They applied a coupled thermo-hygro-chemical (THC) concept which incorporated the reactive water transport equation to estimate the healing motion. The influence of the crack width and quantity of unhydrated cement on material behavior was also studied. Caggiano et al. [2017] presented a coupled damage-plasticity zero-thickness interface theory to simulate concrete cracking and behavior with self-healing consideration. Flow theory of plasticity and continuum damage theory was formulated for the interface model. Fracture energy-based method was employed to control post-peak response for both of failure mode I and II. The constitutive law of interface was extended to take into account of self-healing mechanism on strength, stiffness and post-peak behavior for all fracture modes.

A new method with two phases micro-mechanical model to simulate self-healing mechanism in cementitious materials was developed by Davies and Jefferson [2017]. They used Mori-Tanaka homogenisation along with an Eshelby solution to calculate stress concentration neighboring on inclusions. circular cracks were employed to mimic the microcracks, while solidification formulation was used to incorporate self-healing into the model. The idealised of components in cementitious material along with microcracks can be seen in Fig. 2.8 Healing process in the model was inserted by restoring stiffness of the damaged component along with solidification strain. The healing of microcracks in cementitious material is illustrated in Fig. 2.9 and the current local stress is given by:

$$
S_{L h}=(1-\omega) D_{L}: \varepsilon_{L h}+h \omega_{t h} D_{L h}:\left(\varepsilon_{L h}-\varepsilon_{s}\right)
$$

Where $S_{L h}$ and $\varepsilon_{L h}$ are the equivalent local stress and strain tensor after healing, respectively. $D_{L h}$ is the local stiffness of the healed material and $\omega_{t h}$ is the microcracking parameter at the time of healing. The proportion of healing is defined by parameter $h$ ranging from 0 to 1 and the solidification strain $\left(\varepsilon_{s}\right)$ was also 


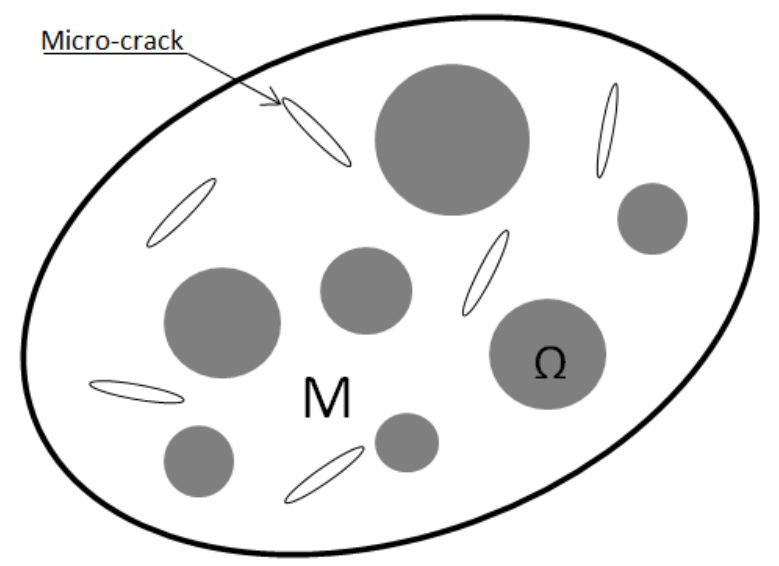

Figure 2.8: Idealised micro-mechanical model of cementitious materials (reproduced from Davies and Jefferson [2017])

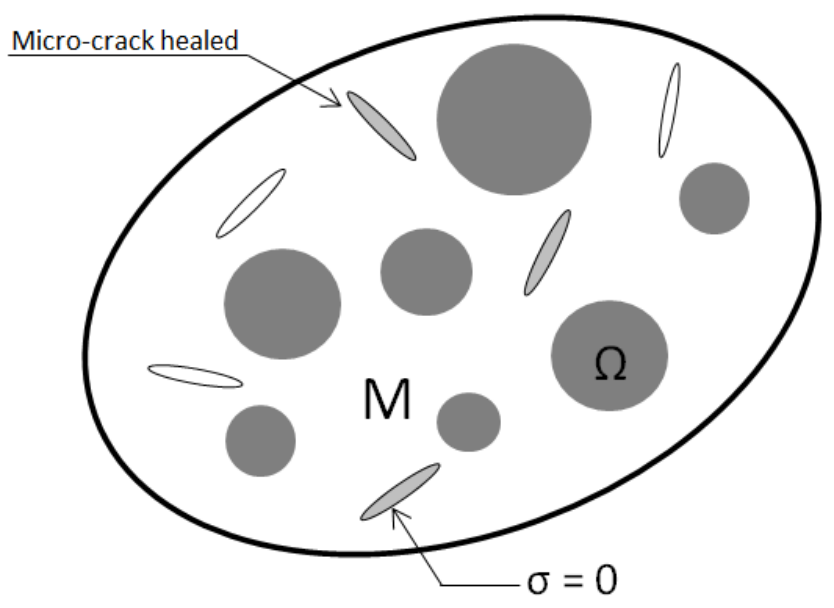

Figure 2.9: Microcracks healed in cementitious materials (reproduced from Davies and Jefferson [2017]) 


\section{Literature Reviews of Concrete Modeling}

incorporated to ensure stress free condition in solidification process of self-healing materials. $D_{L}$ is defined as a $3 \times 3$ matrix contained the non-zero components of the local stiffness tensor and $C_{L}$ is the associated local elastic compliance tensor as follows;

$$
D_{L}=C_{L}^{-1}=E_{M}\left[\begin{array}{ccc}
1 & 0 & 0 \\
0 & \frac{2-\nu_{M}}{4} & 0 \\
0 & 0 & \frac{2-\nu_{M}}{4}
\end{array}\right]
$$

where $D_{L h}=B \cdot D_{L}$ and $B$ is the ratio of material parameter between the stiffness of the healed and original material.

Ranaivomanana and Benkemoun [2017] studied the complex interaction between advection-diffusion mechanism to describe the healing process induced by carbonation in concrete structures. Partial differential equations in the form of transport-reaction for both crack and matrix were used. These equations were discretized by Embedded Finite Element Method (E-FEM) with applying a weak discontinuity in the concentration of calcium ions $\left(\mathrm{Ca}^{2+}\right)$ where the crack is exist. In addition, the calcite layer width, which resulted from healing process, was analytically solved. Softening-healing law based on time-dependent technique was introduced by Zhang and Zhuang [2018] to study self-healing phenomenon in concrete. A mixed-mode traction-separation law was used to consider the relationship between crack opening and traction of two surfaces of crack. They considered crack opening of mode I and II for 2D simulation. Back analysis from self-healing experimental works (three point bending test) was applied to obtain some healing paramaters of healing agents. In order to evaluate the reliability of the model, strong discontinuity approach (SDA) based on standard Statically Optimal Symmetric (SOS) was adopted in their study.

Ramadan Suleiman and Nehdi [2017] developed a hybrid approach of genetic algorithm-artificial neural network (GA-ANN) to predict the autogenous selfhealing phenomenon in concrete. They implemented genetic algorithm in the network as an optimizing tool to assist in reaching global optimum and at the same time local optima could be avoided. Artificial neural network (ANN) was implemented to capture the interdependent parameters in multi difficulties since self-healing concrete is high complex processes. The architecture of network consists of 11 input neurons representing the main parameters influencing the self- 


\section{Literature Reviews of Concrete Modeling}

healing of concrete for example the cement content, water-to-cement ratio (w/c), supplementary materials in matrix, healing materials, crystalline additives, along with 14 neurons as one hidden layer. The model output with a single neuron representing the crack width as an efficiency of self-healing process. The schematic of genetic algorithm-artificial neural network (GA-ANN) is illustrated in Fig. 2.10. The proposed GA-ANN model was validated based on the built database from various experimental studies. The accurate prediction between the proposed algorithm and experimental results is achieved since the results of both of values are close.

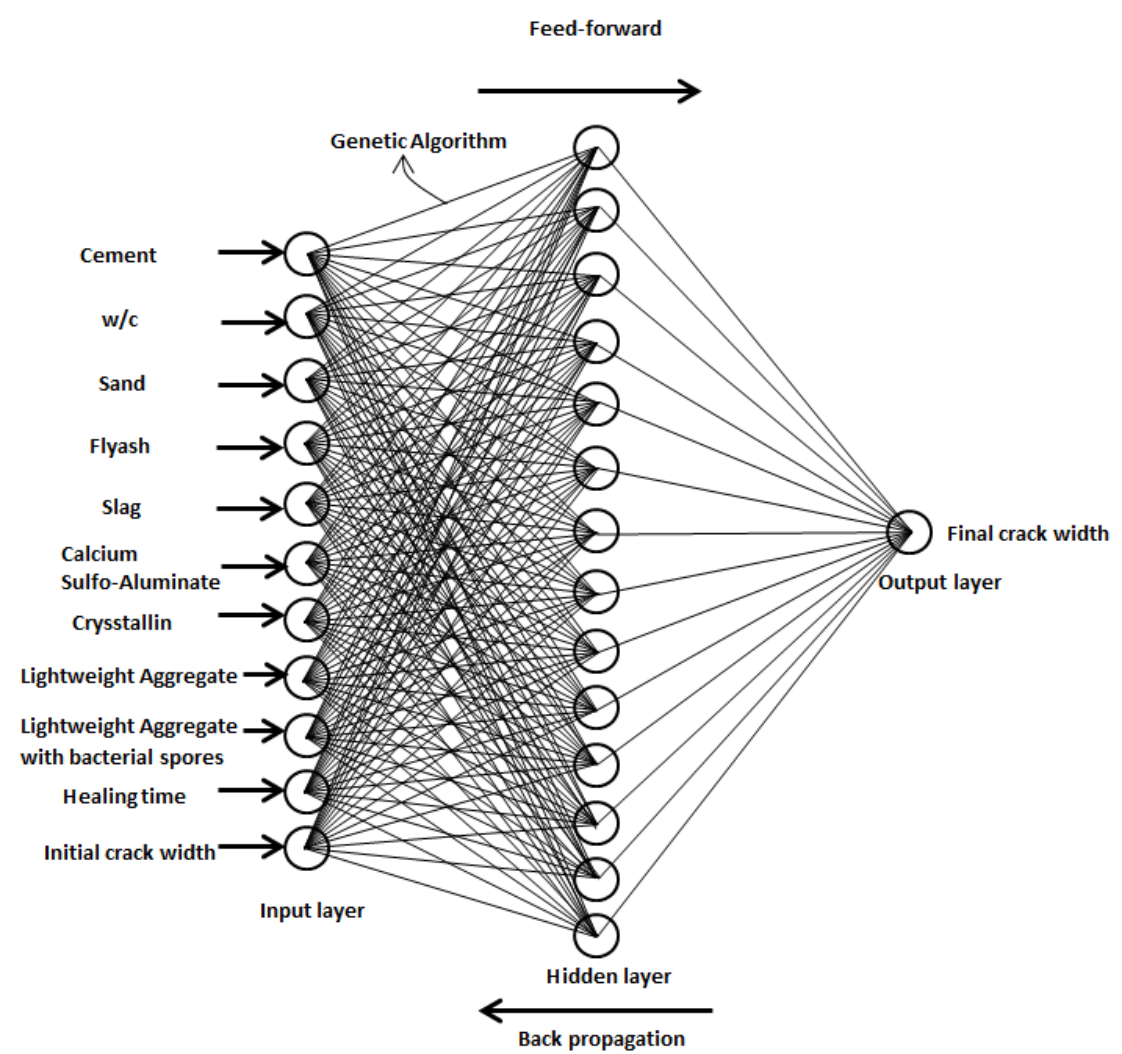

Figure 2.10: The diagram of genetic algorithm-artificial neural network (GAANN) model (reproduced from Ramadan Suleiman and Nehdi [2017]) 


\section{Literature Reviews of Concrete Modeling}

\subsubsection{Autonomous healing model}

The autonomous healing in concrete-based material can be achieved with releasing of encapsulated healing agents caused by some trigger mechanism. The trigger mechanism to obtain healing action which have been found in the literature are ingress of liquids or gasses, exertion of heat or by crack formation [Van Tittelboom and De Belie, 2013]. This section focuses on modeling of self-healing concrete by embedding capsules or microcapsules in the mortar matrix. Zemskov et al. [2014] developed a mathematical model for bacterial self-healing of cracks in concrete structures. They embedded spherical clay capsules along with nutrients for bacteria in the concrete structures. The type of healing agent is calcium lactate which is stored inside the clay capsules. The appearance of crack initiates the breakage of capsule and releasing its content, combining with the entering water could activate the bacteria and converts calcium lactate into calcium carbonate (limestone). Releasing healing agents from the capsule and the calcium carbonate conversion were modelled with a moving boundary problem. A level set method was used to track the moving boundaries, while the diffusion equations was solved with Galerkin finite element method. The proposed mathematical model considered combination of some important parameters, such as crack width and capsule size. The results showed that the crack healed completely. The concentration of calcium lactate dissolved around $60 \%$ before healing is finished. The mathematical model for bacterial self-healing of crack can be easily extended to 3D model since symmetry considerations.

The probability model of penny-shaped cracks hitting the capsules in 2D and 3D model were presented by Lv and Chen [2013]. They developed analytical models on determining capsules dosage embedded in self-healing materials with some targeting healing level. The risk-based probabilistic approach was employed to predict the amount of capsules required to fulfill the particular degree of healing as given by:

$$
P=1-\exp \left[-\sigma\left(\pi R^{2}+2 R l\right)\right]
$$

or expressed in the term of fraction area of capsules $A_{A}$

$$
P=1-\exp \left[-A_{A}\left(1+\frac{2 l}{\pi R}\right)\right]
$$




\section{Literature Reviews of Concrete Modeling}

Where $A_{A}=\pi R^{2} \sigma$. It means that if the expected healing probability $P$ is determined (in their case was intersecting probability), the required dosage of capsules can be calculated as follows:

$$
A_{A}=\frac{\pi R}{\pi R+2 l} \ln \frac{1}{1-P}
$$

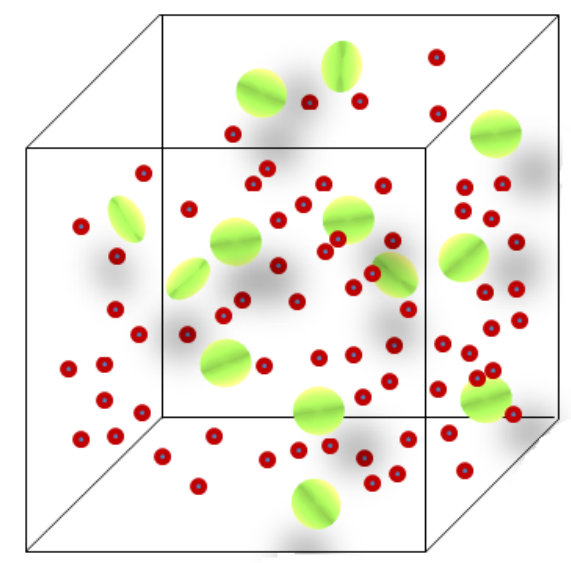

Figure 2.11: 3D self-healing model with spherical capsules and penny-shaped cracks (reproduced from Lv and Chen [2013])

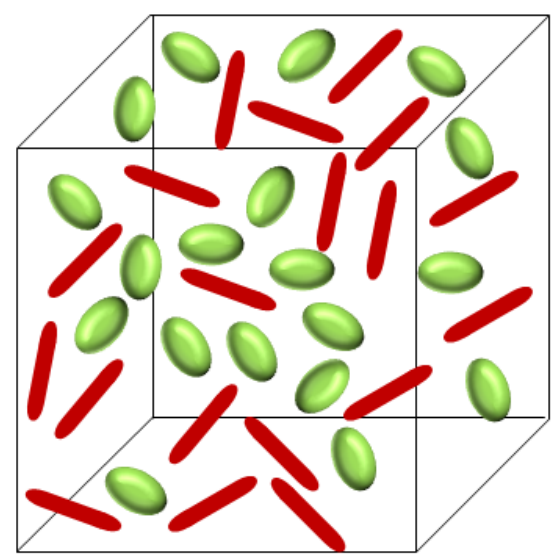

Figure 2.12: 3D self-healing model with cylindrical capsules and penny-shaped cracks (reproduced from Lv and Chen [2013]) 


\section{Literature Reviews of Concrete Modeling}

They also investigated the hitting probability on both of spherical and cylindrical capsules with particular aspect ratio as illustrated in Fig. 2.11 and Fig. 2.12. The proposed probability model was then compared to the analytical solutions based on different dosage of capsules and ratio of crack length to capsule radius. The results showed the in line agreement between simulation and analytical results. The stable value of simulated results are achieved when increasing the number of executions.

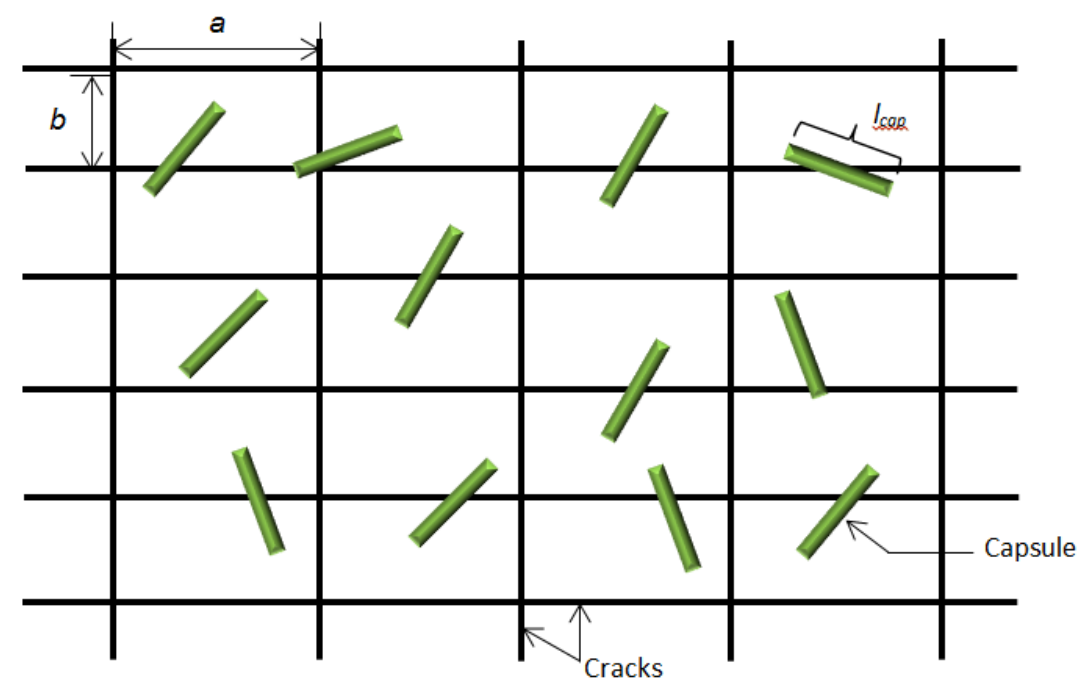

Figure 2.13: 2D simplified cell-like crack model pattern (reproduced from Lv et al. [2014])

In addition, Lv et al. [2014] developed simplification of complex crack patterns due to various mechanism in cementitious materials into linear crack and planar crack patterns in 2D and 3D model, respectively. They assumed the average spacing between adjoining cracks is less than the length of the embedded capsules. With this assumption, the analytical solutions of predicted dosage of capsules required in each type of crack pattern models can be determined using geometrical probability. Some of the crack pattern models in $2 \mathrm{D}$ and $3 \mathrm{D}$ can be seen in Fig. 2.13 and 2.14. The reliability of simulation results both of probability and dosage models closely match to the analytical solutions. The intersecting probability of crack hitting the capsules more stable as the number of capsules increased. Moreover, the probability of healing ratio reaches a stable value, 1 , 


\section{Literature Reviews of Concrete Modeling}

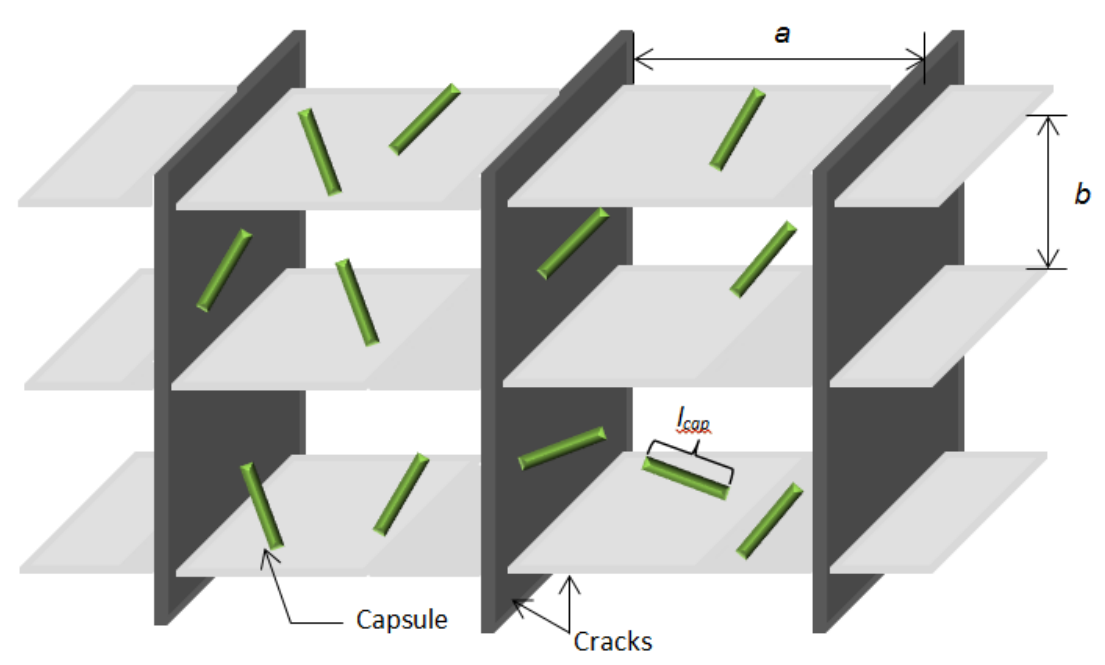

Figure 2.14: 3D simplified orthogonal planar crack model pattern (reproduced from Lv et al. [2014])

increases as the size of sampling increases.

In order to study the optimum microcapsule parameters which would have best effects on self-healing, Zhu et al. [2015] proposed 3D analytical self-healing model based on probability point of view. They considered some parameters of spherical microcapsules such as radius of microcapsules, volume fraction of microcapsules, targeted healing efficiency, broken ratio, etc. To verify the proposed analytical healing model, they conducted monte carlo test to examine the influence of some key paramaters on healing probability and efficiency. The role of interface and stress concentration around embedded cylindrical capsules in a matrix was investigated by Gilabert et al. [2015]. They modeled a matrix as an elastic, homogeneous and isotropic which subjected to the uniform and uni-axial stress. The model with square plate of length $2 L$ consists a hole with radius $R c$ and shell thickness $t c$ had been proposed. the quarter specimen with the half-length of square $L=50 \mathrm{~mm}, R c=1.5 \mathrm{~mm}$ and $t c=0.175 \mathrm{~mm}$ had been performed because of symmetry as shown in Fig. 2.15 and 2.16. The optimum combination properties, the effects of variation of elastic properties between matrix and capsule, different thickness of capsules and bonding strength on generation of stress concentration around capsule had been studied. The results showed that using a soft capsule $(E c / E m<1 / 5)$ restore the mechanical response governed by 


\section{Literature Reviews of Concrete Modeling}

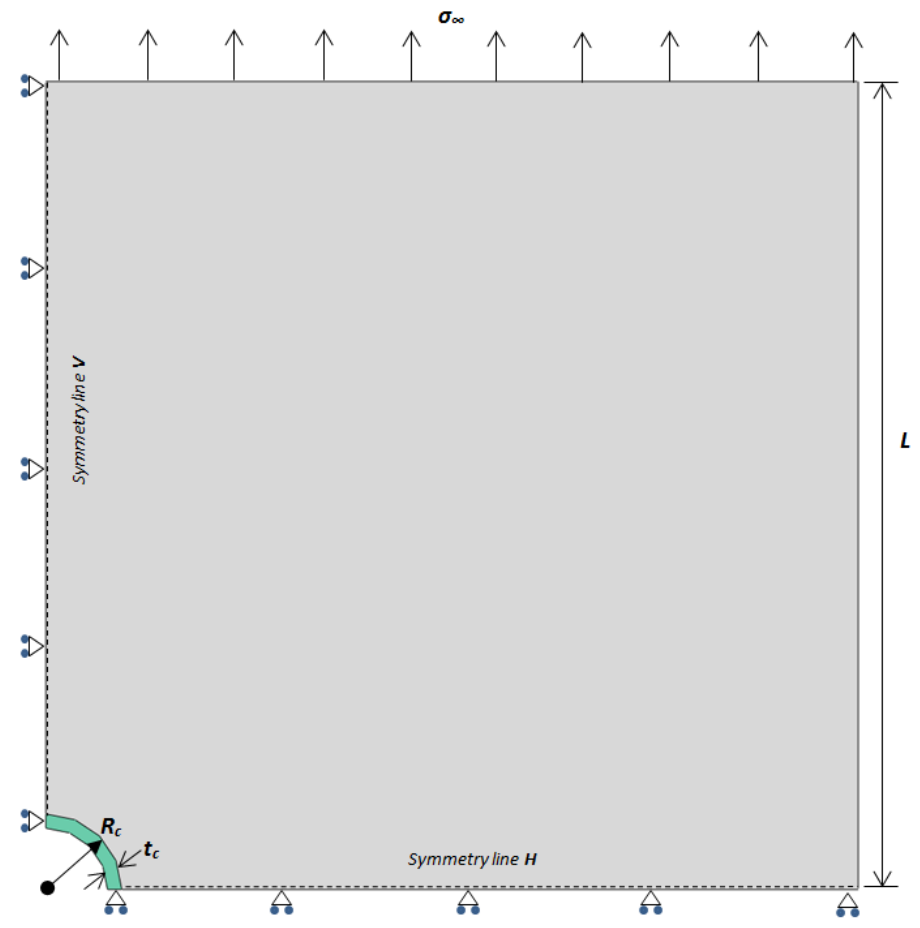

Figure 2.15: Schematic of numerical model-Geometry, boundary conditions and uniaxial loads (reproduced from Gilabert et al. [2015])

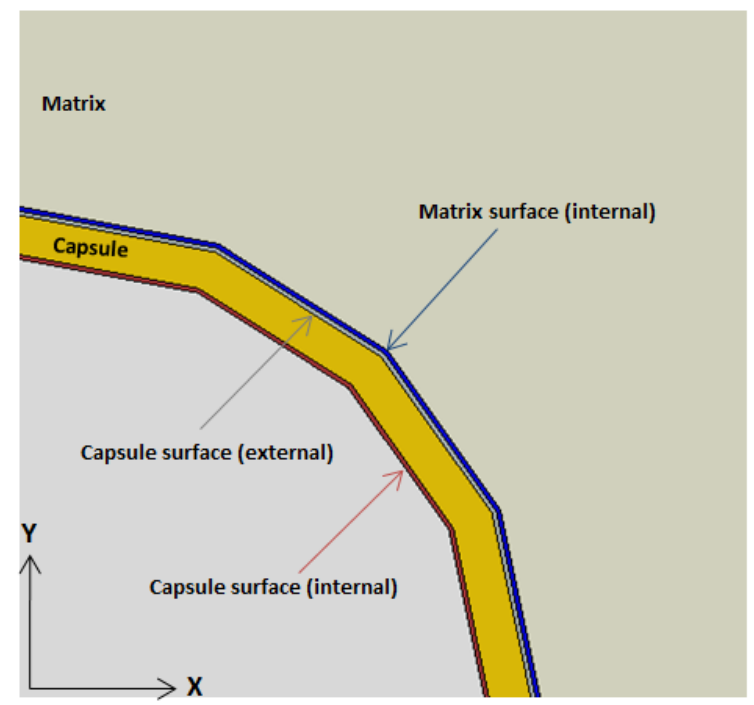

Figure 2.16: Detail surfaces between capsule and matrix (reproduced from Gilabert et al. [2015]) 


\section{Literature Reviews of Concrete Modeling}

Kirschs equations for a plate with a hole. Moreover, in order to avoid debonding phenomenon, the stress required depends linearly on the logarithm of geometric ratio $(t c / R c<0.6)$. Huang and Ye [2016] investigated the effects amount of waters supplied with capsules to the healing efficiency due to further hydration of unhydrated cement. The efficiency of the amount supplied water in capsules determined numerically as function capsule dosages and sizes. The probability of a crack hitting capsules was simulated with a beam model with the size of $40 \mathrm{~mm} \mathrm{x}$ $40 \mathrm{~mm}$ x $160 \mathrm{~mm}$. As shown in Fig. 2.17. They assumed all random distribution capsules inside the beam were spherical with identical radius, no overlapping, and could not cross the edge of beam. The cement paste in the model was simplified

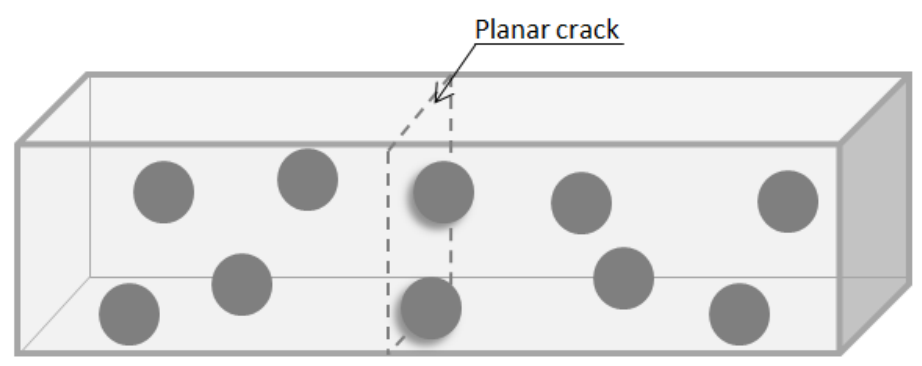

Figure 2.17: Diagram of planar crack hitting capsules model (reproduced from Huang and Ye [2016])

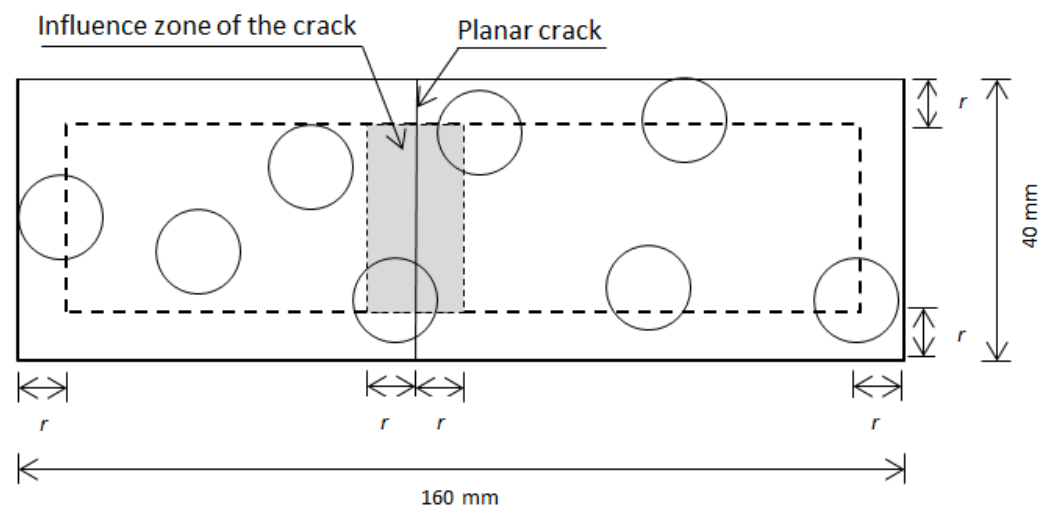

Figure 2.18: Schematic distribution of capsules and the influence zone of planar crack (reproduced from Huang and Ye [2016])

as homogeneous material and the crack was simplified as planar crack. In their 


\section{Literature Reviews of Concrete Modeling}

model, the probability of crack hitting the capsules is defined as the probability of capsules center across the influence zone of the crack as illustrated in Fig. 2.18. To obtain this, statistical analysis of 1000 random samples were conducted. The numerical results found that the increasing amount of released water in line with the dosage of capsules at different slops. The models with dosage capsules $3 \%$, $5 \%$, and $7 \%$ have optimum size of capsule is $6.5 \mathrm{~mm}$ from self-healing efficiency point of view. The mechanical properties of cementitious materials (elastic modulus and tensile strength) decrease as the volume fraction of capsules increases. The breaking mechanism of microcapsules in concrete due to microcracking was studied by Zhou et al. [2016]. One circular microcapsule as void and one microcrack were considered in the model. They simulated the fracture behavior of concrete specimens using two-dimensional particle flow code (PFC2D). Moreover, Zhou et al. [2017] proposed 3D damage-healing of encapsulation-based self-healing system using discrete element method (DEM) under compression load. They investigated the effects of some key parameters on encapsulation-based self-healing materials, such as the strength and stiffness of solidified healing agent, the initial damage and the partial effect of healing. The specimen was generated using PFC3D with the radius $5.08 \mathrm{~cm}$ and height $20.32 \mathrm{~cm}$, and all spheres particles are connected by the parallel bond model as illustrated in Fig. 2.19. They proposed scalar healing variable $h$ as a negative damage to take into account in the existing damage model. In the discrete element method, it is assumed that the number of bonds rises after healing right after damage occurred as shown in Fig. 2.20. Based on discrete element method, the scalar healing parameter $h$ can be simplify determined by

$$
h=\frac{N_{h}-N_{d}}{N_{i}-N_{d}}, 0 \leq h \leq 1
$$

Where $N_{h}$ is the number of bonds after healing, $N_{d}$ is the number of bonds after damage, and $N_{i}$ is the number of initial bonds. if $N_{h}=N_{d}$, it means that healing is not occured or $h=0$. Identically, if $N_{h}=N_{i}$, it means that all damaged bonds are healed or $h=1$. To obtain self-healing effect, $N_{h}$ should be higher than $N_{d}$. The DEM under compression load model was calibrated by available experimental results. The numerical results showed that the fluctuation at post-peak 


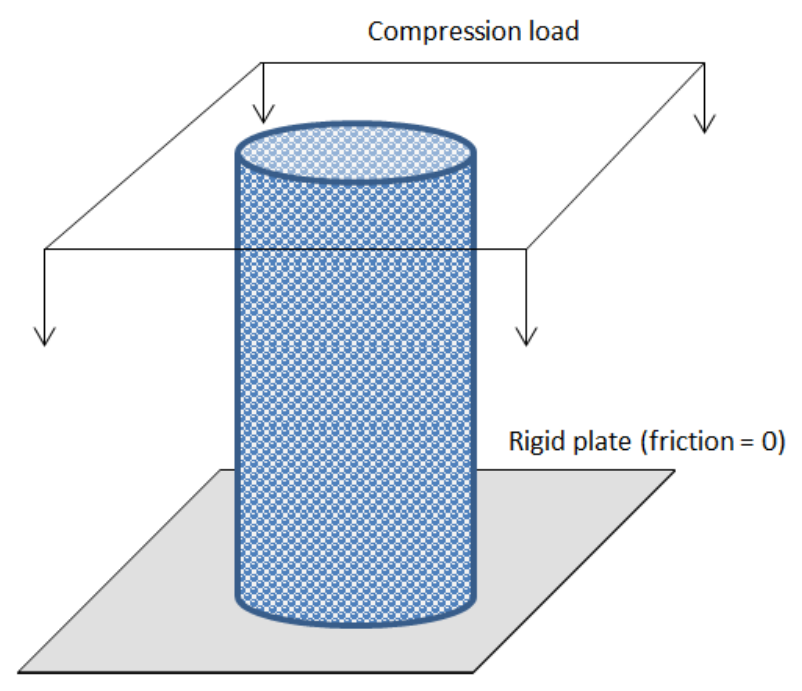

Figure 2.19: PFC3D model under compression loading (reproduced from Zhou et al. [2017])

(a)

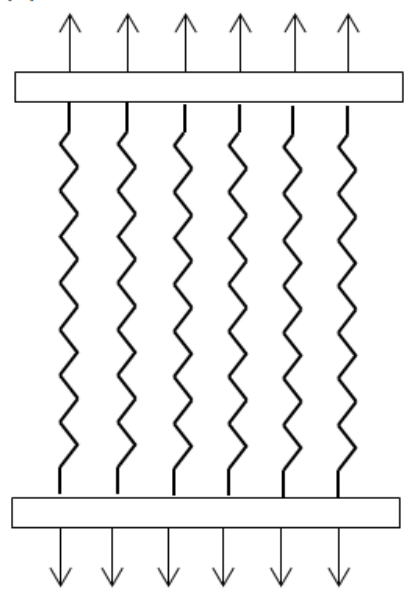

(b)

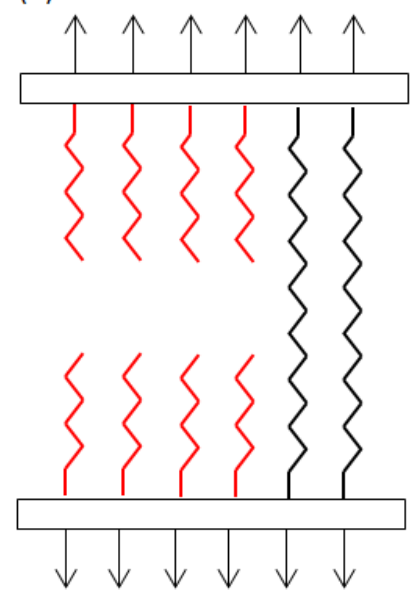

(c)

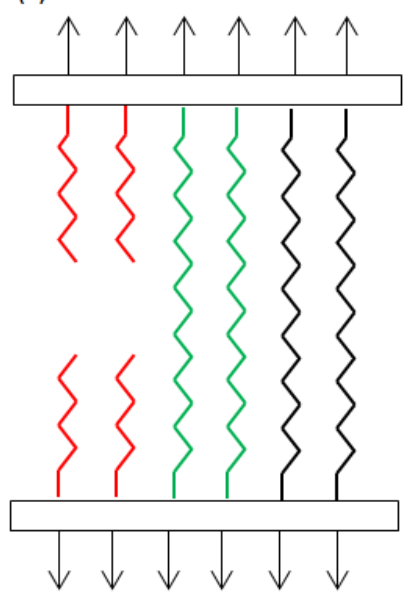

Figure 2.20: Discrete element model with number of bonds. (a) The initial number of bonds (Ni), (b) The number of bonds after damage (Nd), and (c) The number of bonds after healing (Nh) (reproduced from Zhou et al. [2017]) 


\section{Literature Reviews of Concrete Modeling}

regime are observed due to self-healing effects. The compressive strength of the model increases in line with stiffness of solidified healing agent at post-healing. The strength of specimens after healing was influenced by initial damage and the strength of healing agent.

Recently Li et al. [2017] investigated the crack propagation process in a matrix and debonding phenomenon of capsules in encapsulation-based self-healing materials. They used the extended finite element method (XFEM) to model crack propagation combined with the cohesive zone model (CZM) to model interface between capsule and matrix. The 2D model consists of an infinite matrix with capsule as a hole and embedded crack. They investigated the effect of geometry, elastic and fracture properties as parametric studies of the system. The numerical results found that the effect of capsule wall on fracture behavior is not significant for the small ratio of capsule thickness and its outer radius, while the crack propagation rate is strongly dependent on the ratio of elastic modulus $(E c / E m)$. Apart from the aforementioned review, the development of computer simulation and built-in software to study the self-healing process is still limited. This is due to the complexity of self-healing process in the system both of autogenous and autonomous methods. 


\section{Chapter 3}

\section{Numerical Modeling of Encapsulation-Based Self-Healing Concrete}

Recent intense researches in self-healing concrete area have been drawn to an autonomous type of self-healing method using micro-capsules for more accurate healing location and better healing capabilities. The healing agents are placed inside discrete micro-capsules embedded in the substrate material. Approaching crack break the capsule shell to release the healing agent and hence the healing occurs in the vicinity of the damaged part, see Fig. 3.1.
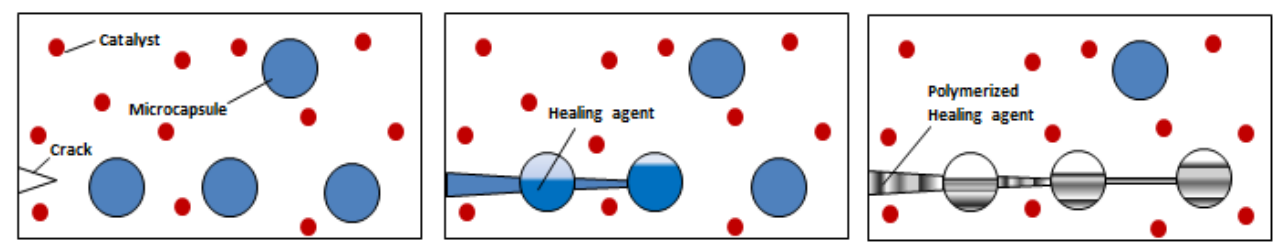

Figure 3.1: Autonomous self-healing using micro-capsules (reproduced from White et al. [2001])

In order to achieve the efficiency of the self-healing mechanism, the understanding of the behavior of different constituents and their interactions plays an important role of this area. In this study, 2D numerical models with randomly packed aggregates and capsules are developed to analyze fracture mechanism that play a 


\section{Numerical Modeling of Encapsulation-Based Self-Healing Concrete}

significant role in the fracture probability of capsules and consequently the selfhealing process. The capsules embedded in self-healing concrete should be able not only to break when the crack appears, but also to resist during mixing process. According to recent experimental works, polymeric capsules made of Poly Methyl Methacrylate-PMMA are among the most efficient healing agents. They are not only brittle enough to break when they are subjected to relatively small deformations but also strong enough to resist during the mixing process compared to glass capsule, poly lactic acid (PLA), and polystyrene (PS) [Hilloulin et al., 2015]. Hence, this study focuses on these polymeric capsules (Poly Methyl Methacrylate-PMMA) which are uniformly distributed in the mortar matrix. The healing agent (methyl methacrylate) is modelled as 'solid'. Hence, the flow process and the associated polymerization of the healing agent into the crack surface and the associated healing efficiency in vicinity of the damage is not addressed here but will be investigated on the future studies. The voids and interfacial cohesion around the constituents affect the healing process. The strong adhesive between capsule and matrix will forced the capsule to break [Alexeev et al., 2007]. In order to model the complicated fracture processes in this multi-phases specimens, cohesive elements were inserted between the mortar-, aggregates-, capsule cores- and capsule walls-elements and at their phase interfaces.

\subsection{Microstructures generation at mesoscale level}

\subsubsection{Aggregates and capsules generation}

Concrete is comprised of cement (10-15\%), coarse and fine aggregates (60-75\%) and water (15-20\%). For normal strength concrete, coarse aggregates usually represent about $30-50 \%$ of the concrete volume. In order to describe the distribution of the aggregates, the Fuller curve is often used, categorizing the aggregates into a certain number of segments based on the sieve analysis. The aggregate size distributions found in Hirsch [Hirsch, 1962] and summarized in Table 3.1 are employed in this study. The sieve size of $2.36 \mathrm{~mm}$ is taken as the cut-off size of coarse aggregates. Concrete is treated as a four-phases materials consisting of aggregates, mortar, capsule cores and capsule shells. According to X-ray tomo- 


\section{Numerical Modeling of Encapsulation-Based Self-Healing Concrete}

Table 3.1: Aggregate size distribution[Hirsch, 1962]

\begin{tabular}{llll}
\hline $\begin{array}{l}\text { Sieve } \\
(\mathrm{mm})\end{array}$ & size & $\begin{array}{l}\text { Total percent- } \\
\text { age retained } \\
(\%)\end{array}$ & $\begin{array}{l}\text { Total percent- } \\
\text { age passing } \\
(\%)\end{array}$ \\
\hline 12.70 & 0 & 100 & \\
9.50 & 39 & 61 \\
4.75 & 90 & 10 \\
2.36 & 98.6 & 1.4 \\
\hline
\end{tabular}

graphic images, the coarse aggregates are often of circular and elliptical shape [Daudeville, 2014]. It was also found that the influence of other shapes barely influence the macroscopic behavior of concrete [Du et al., 2014]. Therefore, for the sake of simplicity, circular capsules and aggregates are generated and used in our studies. The size and thickness of capsules can vary according to the agitation rate of experiments. Recent experiments showed that the range of diameter size of capsules is between $1.1 \mathrm{~mm}-7.2 \mathrm{~mm}$ [Hilloulin et al., 2015] and $1.7 \mathrm{~mm}-2.3$ $\mathrm{mm}$ [Gruyaert et al., 2016]. In this study, the preliminary model will use $2.0 \mathrm{~mm}$ as diameter of the capsules and the capsule wall thickness is considered as diameter/27 (from real capsule thickness/diameter ratio [Van Tittelboom et al., 2011]). In addition, we test the influence of the volume fraction of capsules ranging from $1.57 \%$ to $9.42 \%$ as summarized in Table 3.2 .

Table 3.2: Volume fraction of capsules used in the model

\begin{tabular}{lllll}
\hline Model & $\begin{array}{l}\text { Diameter } \\
(\mathrm{mm})\end{array}$ & $\begin{array}{l}\text { Thickness of } \\
\text { capsule shell } \\
(\mathrm{mm})\end{array}$ & $\begin{array}{l}\text { Number } \\
\text { capsules }\end{array}$ & $\begin{array}{l}\text { of } \\
\text { Microcapsules } \\
\text { volume frac- } \\
\text { tion (\%) }\end{array}$ \\
\hline Cap_0 & - & - & 0 & 0 \\
Cap_50 & 2.0 & 0.07 & 50 & 1.57 \\
Cap_100 & 2.0 & 0.07 & 100 & 3.14 \\
Cap_200 & 2.0 & 0.07 & 200 & 6.28 \\
Cap_300 & 2.0 & 0.07 & 300 & 9.42 \\
\hline
\end{tabular}




\section{Numerical Modeling of Encapsulation-Based Self-Healing Concrete}

\subsubsection{Packing algorithms}

The commercial finite element package ABAQUS is employed in all simulations. In order to randomly disperse the aggregates and capsules in the matrix, a packing algorithm to generate and distribute all microstructures into the mortar matrix has been developed [Quayum et al., 2015]. The basic idea of the algorithm is to create randomly distributed aggregates and capsules in the concrete repeatedly until the target area is filled with aggregates and capsules. The generation starts with randomly processing the different aggregates within the size range and the grading segments, and then randomly packing them into the prescribed region. With the same algorithm, the specific diameter and number of capsules are generated. There are some specific constraints to be satisfied simultaneously:

1. The whole inclusion (aggregates and capsules) must be within the concrete.

2. Overlapping between inclusions are not allowed and

3. there is no intersection between two inclusions.

A series of concrete samples with dimensions of $100 \mathrm{~mm} \times 100 \mathrm{~mm}$ in 2D are generated. Different volume fraction of capsules, aggregate volume fraction of $40 \%$ and minimum space between the edge of an aggregate and the boundary of the concrete specimen equal $0.5 \mathrm{~mm}$ are adopted here according to Table 1 . In order to ensure statistical convergence, for each volume fraction of capsules, some random samples with different realizations are also generated. Fig. 3.2 shows the $2 \mathrm{D}$ concrete specimen with different volume fraction and constant aggregate volume fraction of $40 \%$.

\subsection{Cohesive zone model}

\subsubsection{Cohesive elements}

The samples are meshed with T3 elements assuming plane stress conditions. Different simulations have been carried out with different mesh sizes ranging from $0.5 \mathrm{~mm}$ to $1.0 \mathrm{~mm}$ in order to ensure that the results are independent of the discretization. 4-node cohesive interface elements (CIE) are inserted into the 
3. Numerical Modeling of Encapsulation-Based Self-Healing Concrete

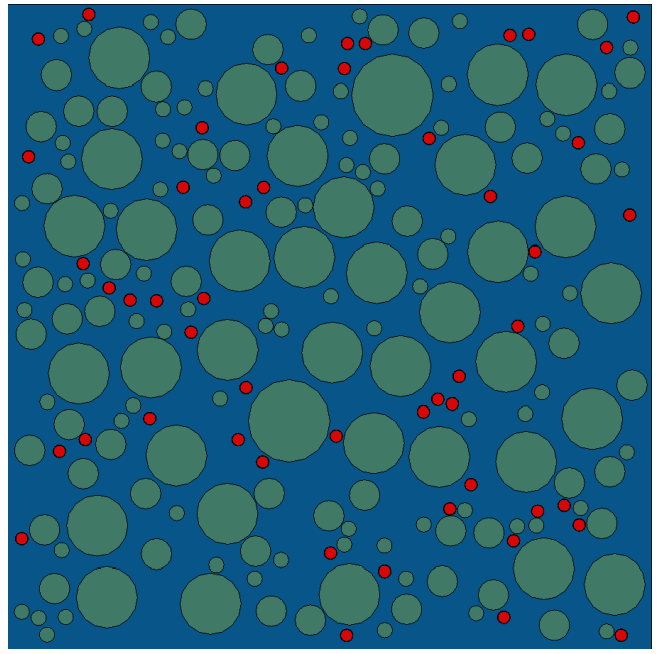

(a) Cap_50 (Vf=1.57\%)

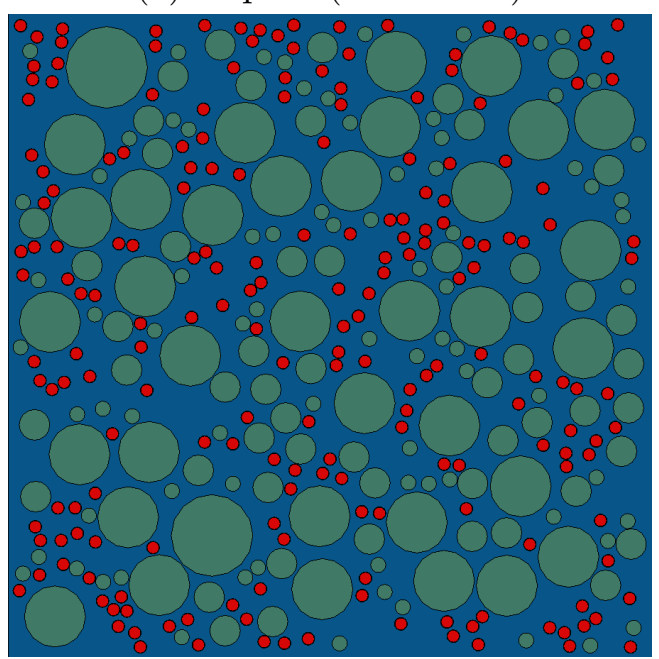

(c) Cap_200 (Vf=6.28\%)

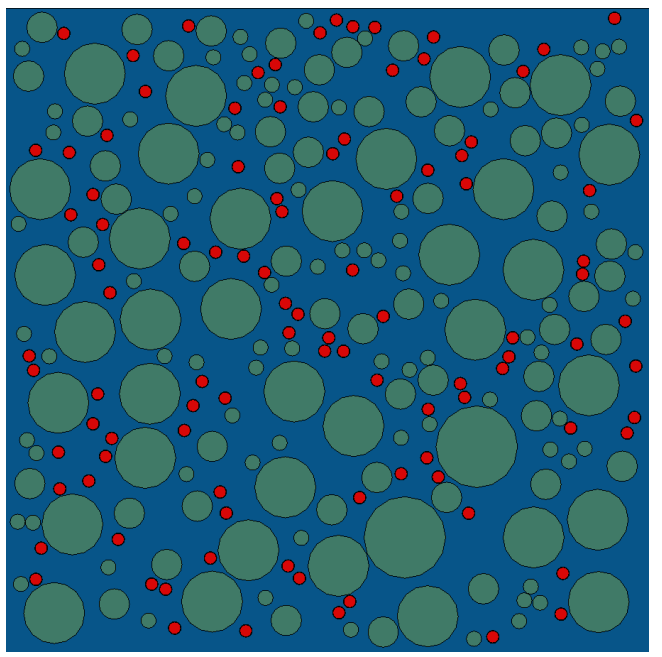

(b) Cap_100 (Vf=3.14\%)

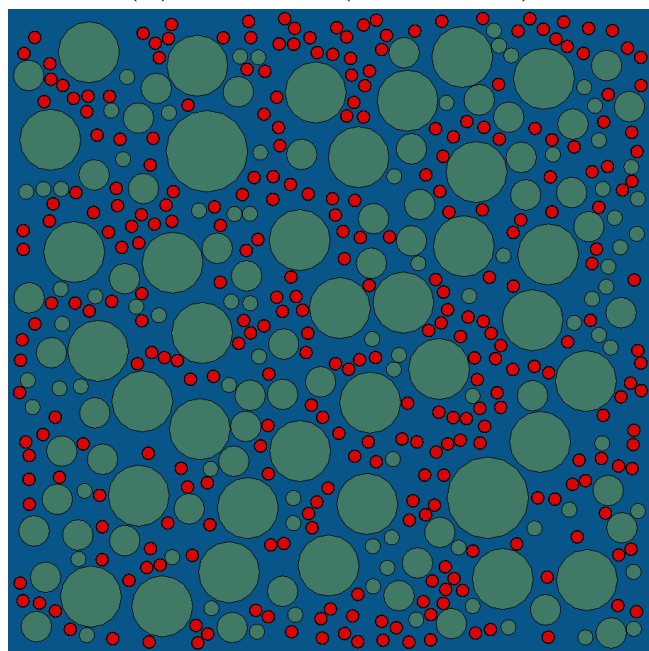

(d) Cap_300 (Vf=9.42\%)

Figure 3.2: Mesoscale model of encapsulation-based self-healing concrete at consistent volume fraction of aggregates $40 \%$ 


\section{Numerical Modeling of Encapsulation-Based Self-Healing Concrete}

generated initial mesh, i.e. inside the aggregates (CIE-AA), inside the mortar (CIE-MM), inside the capsule cores (CIE-CC), inside the capsule shells (CIESS), between the aggregate and the mortar (CIE-AM), between the capsule cores and the shells (CIE-CS) and between the capsule shells and the mortar (CIE$\mathrm{SM})$. The cohesive elements insertion process is conducted by a Python script in combination with an ABAQUS batch processing. With this method, the new generated mesh will have duplicate nodes at the interfaces automatically and then the 4-node cohesive element (COH2D4) is inserted into the discretization accordingly as illustrated in Fig. 3.3.

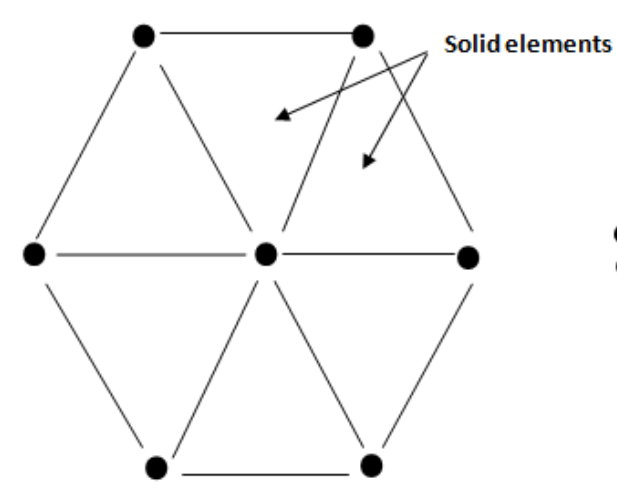

(a)

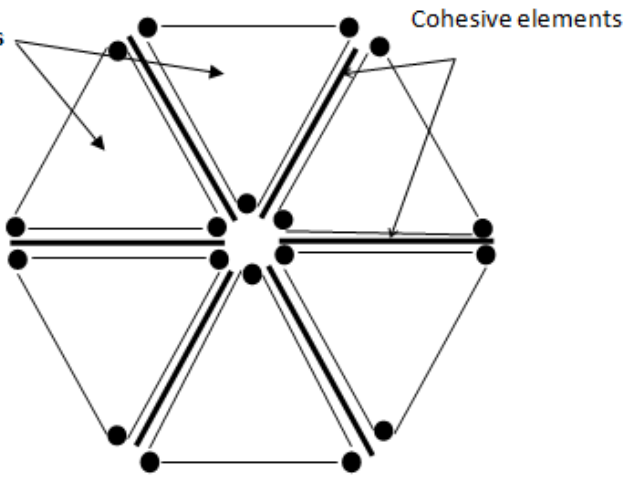

(b)

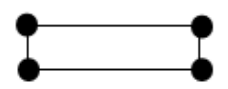

In- plane thickness $=0$

(c)

Figure 3.3: Inserting cohesive elements in the initial mesh. (a) Initial mesh around a node. (b) Mesh with cohesive elements. (c) 2D 4-noded cohesive elements

\subsubsection{Traction separation laws}

The cohesive elements, which are pre-inserted into the initial meshes, are based on the cohesive crack model available in ABAQUS, and its constitutive behavior can be described by tension/shear softening laws illustrated to Fig. 3.4. The cohesive zone model in ABAQUS requires two input parameters, i.e. the fracture energy

$$
G=\int_{0}^{\delta_{f}} t(\delta) d \delta=\frac{1}{2} t_{o} \delta_{f}
$$




\section{Numerical Modeling of Encapsulation-Based Self-Healing Concrete}

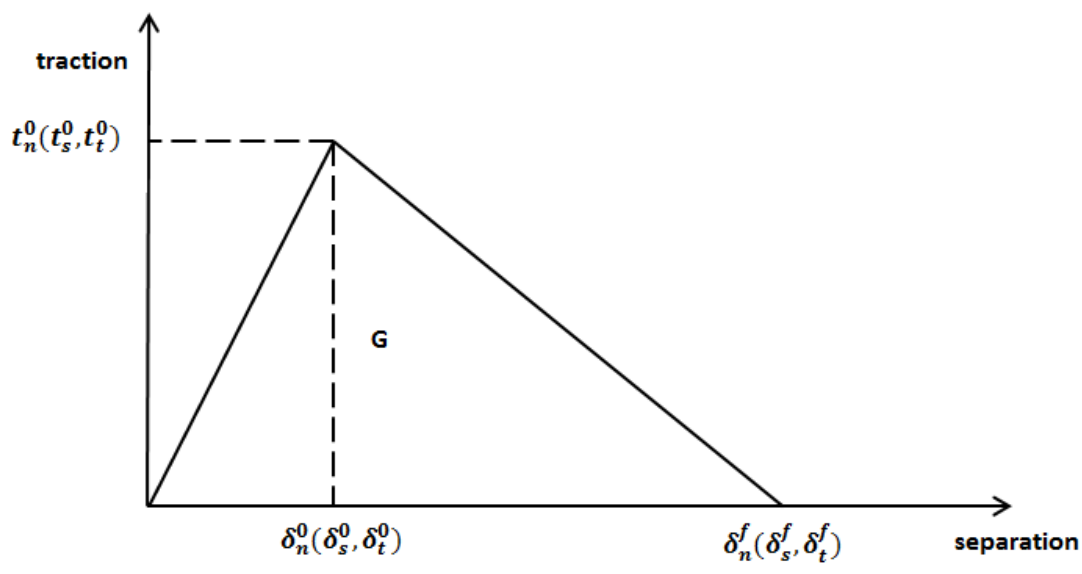

Figure 3.4: bilinear traction-separation law, reproduced from [Simulia, 2013]

and the cohesive strength $t_{o} ; \delta_{f}$ is the separation displacement. The effective relative displacements $\delta_{m}$ can be determined after considering the effects of displacements in both normal $\left(\delta_{n}\right)$ and tangential directions $\left(\delta_{s}\right)$ :

$$
\delta_{m}=\sqrt{\left\langle\delta_{n}\right\rangle^{2}+\delta_{s}^{2}}
$$

where \langle\rangle is the Macaulay bracket and

$$
\left\langle\delta_{n}\right\rangle=\left\{\begin{array}{lll}
\delta_{n}, & \delta_{n} \geq 0 & (\text { tension }) \\
0, & \delta_{n}<0 & (\text { compression })
\end{array}\right.
$$

The damage evolution in this study is determined by a scalar index D given by

$$
D=\frac{\delta_{m f}\left(\delta_{m, \max }-\delta_{m 0}\right)}{\delta_{m, \max }\left(\delta_{m f}-\delta_{m 0}\right)}
$$

where $\delta_{m f}$ is the effective displacements at failure, $\delta_{m, \max }$ is the maximum relative displacement obtained during the loading, and $\delta_{m, 0}$ is the effective displacement at damage initiation. It is obvious that the damage will evolve monotonically from 0 to 1 during loading as indicated in Eq. 3.4. The damage in all cohesive elements is assumed to initiate when a quadratic function including the ratio of 


\section{Numerical Modeling of Encapsulation-Based Self-Healing Concrete}

nominal stress reaches a value one.

$$
\left\{\frac{\left\langle t_{n}\right\rangle}{t_{n 0}}\right\}^{2}+\left\{\frac{t_{s}}{t_{s 0}}\right\}^{2}=1
$$

where $t_{n}$ and $t_{s}$ are the traction in normal and tangential direction, respectively. A typical discretization around the constituents of the self-healing concrete after the insertion of cohesive elements is illustrated in Fig. 3.5.

\subsubsection{Weak form and discretization}

The weak form of the equilibrium equation can be formulated as follows:

$$
\int_{V}(\nabla \mathbf{w})^{c}: \mathbf{S} d V=\int_{V} \nabla \mathbf{w} \cdot \mathbf{q} d V+\int_{A} \mathbf{w} \cdot \mathbf{t} d A+\int_{A}[[\mathbf{w}]] \cdot \mathbf{t}_{c} d A
$$

with gradient operator $\nabla$, stress tensor $\mathbf{S}$, body force vector $\mathbf{q}$, boundary traction $\mathbf{t}, \mathbf{t}_{c}$ cohesive tractions and an arbitrary continuous weighing function $\mathbf{w}$; [[.]] denotes the jump operator. The last term on the RHS refers to the cohesive energy. In contrast to partition-of-unity enriched approaches as proposed in Rabczuk and Samaniego [2008]; Rabczuk et al. [2007a,b, 2008a,b, 2009, 2010a,b,c], the jump term is computed by pairwise nodes in cohesive elements. Therefore, a simple node splitting techniques is employed after a certain fracture criterion is reached. Substituting the finite element discretization of the trial and test functions

$$
\begin{aligned}
\mathbf{u}^{\mathbf{h}}(\mathbf{X}) & =\mathbf{N}(\mathbf{X}) \mathbf{d} \\
\mathbf{w}^{\mathbf{h}}(\mathbf{X}) & =\mathbf{N}(\mathbf{X}) \mathbf{w}
\end{aligned}
$$




\section{Numerical Modeling of Encapsulation-Based Self-Healing Concrete}

into the weak form leads finally after linearization the following well-known system of equations:

$$
\begin{aligned}
\mathbf{K D} & =\mathbf{F}_{\text {ext }}-\mathbf{F}_{\text {int }} \\
\mathbf{K} & =\int_{V} \mathbf{B}^{T} \mathbf{C}^{t a n} \mathbf{B} d V \\
\mathbf{F}_{\text {int }} & =\int_{V} \mathbf{B}^{T} \mathbf{S} d V \\
\mathbf{F}_{\text {ext }} & =\int_{A} \mathbf{N}^{T} \mathbf{q} d A+\int_{A} \mathbf{N}^{T} \mathbf{t} d A+\int_{A} \mathbf{N}^{T} \mathbf{t}_{c} d A
\end{aligned}
$$

where $\mathbf{N}$ is the vector contain the finite element shape functions and the vectors $\mathbf{d}$ and $\mathbf{w}$ contain the nodal parameters of the displacement field and virtual displacement field which are identical to the physical values in FEM in contrast to other approaches such as meshless methods [Belytschko et al., 1994a; Nguyen et al., 2008] or isogeometric analysis [Ghasemi et al., 2017; Ghorashi et al., 2015; Hughes et al., 2005; Nguyen et al., 2015; Nguyen-Thanh et al., 2011a,b, 2015]. The matrix $\mathbf{B}$ contains the spatial derivatives of the shape functions. The nonlinear system of equations is solved with Newton method. 


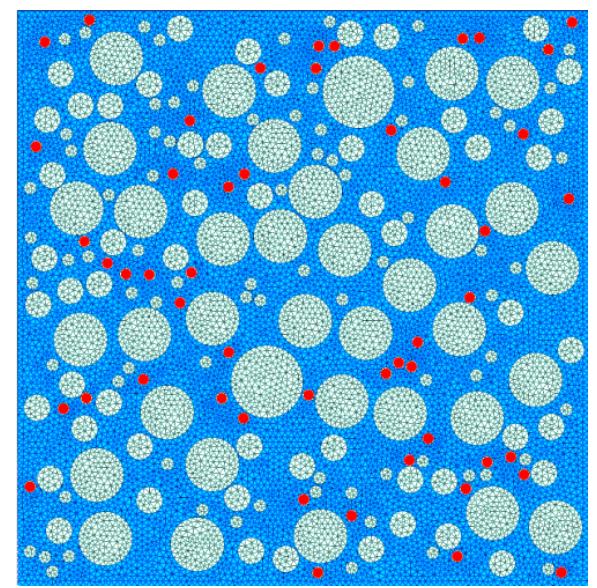

(a)

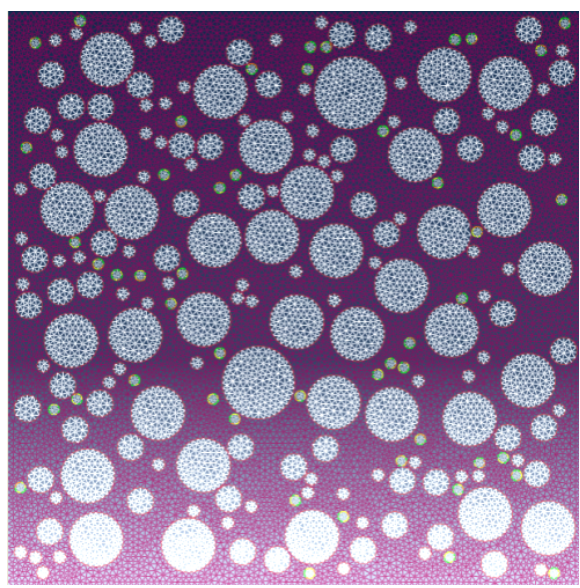

(b)

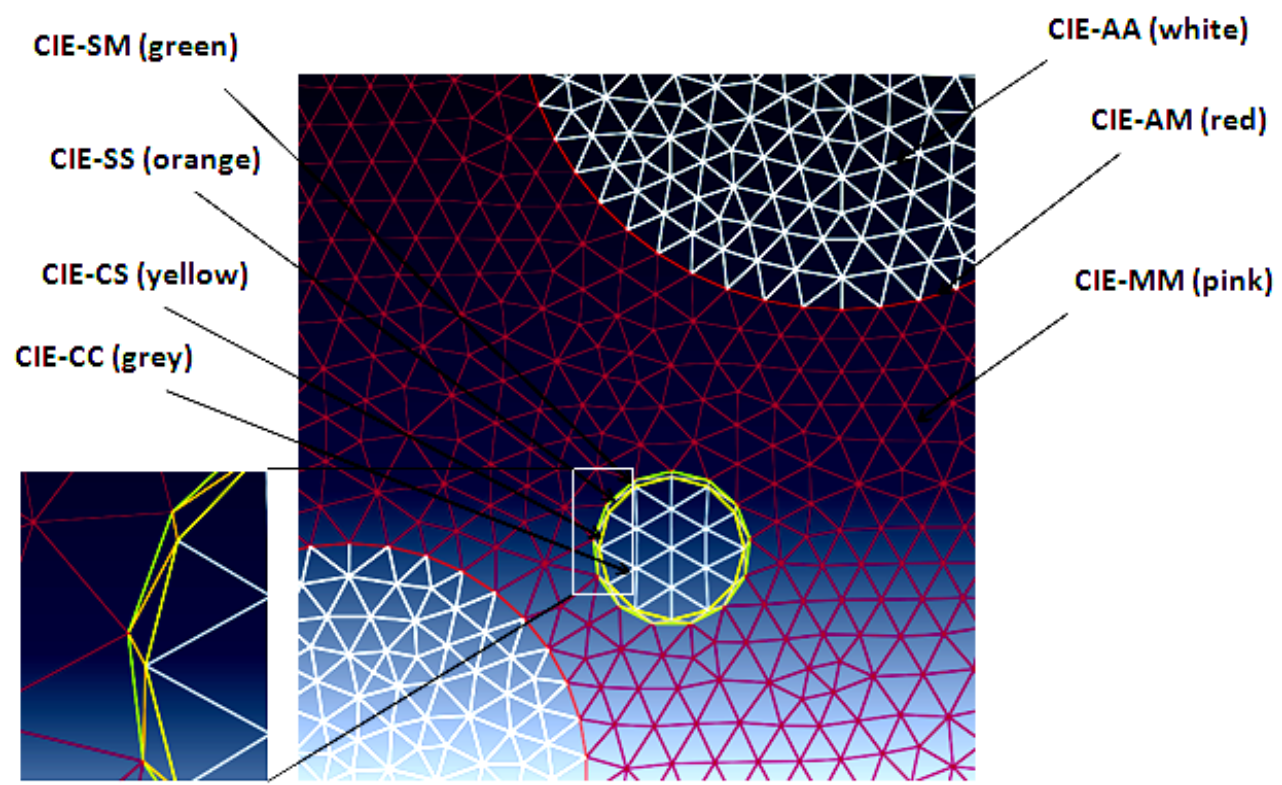

(c)

Figure 3.5: Generation of finite element meshes. (a) Initial mesh. (b) Cohesive elements around the sample. (c) Seven sets of cohesive interface elements are automatically generated, namely, inside the aggregates (CIE-AA), inside the mortar matrix (CIE-MM), inside the capsule shells (CIE-SS), inside the capsule cores (CIE-CC), the aggregate and the mortar matrix interfaces (CIE-AM), the capsule cores and the shells interfaces (CIE-CS), and the capsule shells and the mortar interfaces (CIE-SM) 


\section{Chapter 4}

\section{D Numerical Simulations Under Uniaxial Tension}

\subsection{Model parameter}

The 2D specimens with dimensions of $100 \mathrm{~mm}$ x $100 \mathrm{~mm}$ are loaded under uniaxial tension assuming plain stress conditions. The geometry, boundary, and loading conditions of the specimen are illustrated in Fig. 4.1. The experiment was done displacement-controlled up to a displacement $\mathrm{d}=0.1 \mathrm{~mm}$ or strain $=0.001$. The solid elements for aggregates, mortar, and capsules (CPS3) were assumed to behave linear elastic. All material properties including density, Young's modulus, Poisson's ratio, tensile strength, and fracture energy for the aggregates, mortar, capsules and different interface cohesive elements (as explained in section 3.2) are listed in Table 4.1. Capsules containing the healing agent, i.e. methyl methacrylate (MMA), are considered in this study. A small concentration of PMMA is added to increase the viscosity of the healing agent as discussed in [Van Tittelboom et al., 2011]. Hence, the modulus of elasticity (E) of the healing agent is considered lower than the one from [Matbase]. Considering that the healing agent behaves like a liquid material, the Poisson's ratio of healing agent should be near to 0.5. So, the value 0.45 is chosen for this study. However, for the sake of simplicity, the fluid flow of the healing agent is not considered as the focus of this study is on the breakage of the capsules and capsule shells. The properties of 


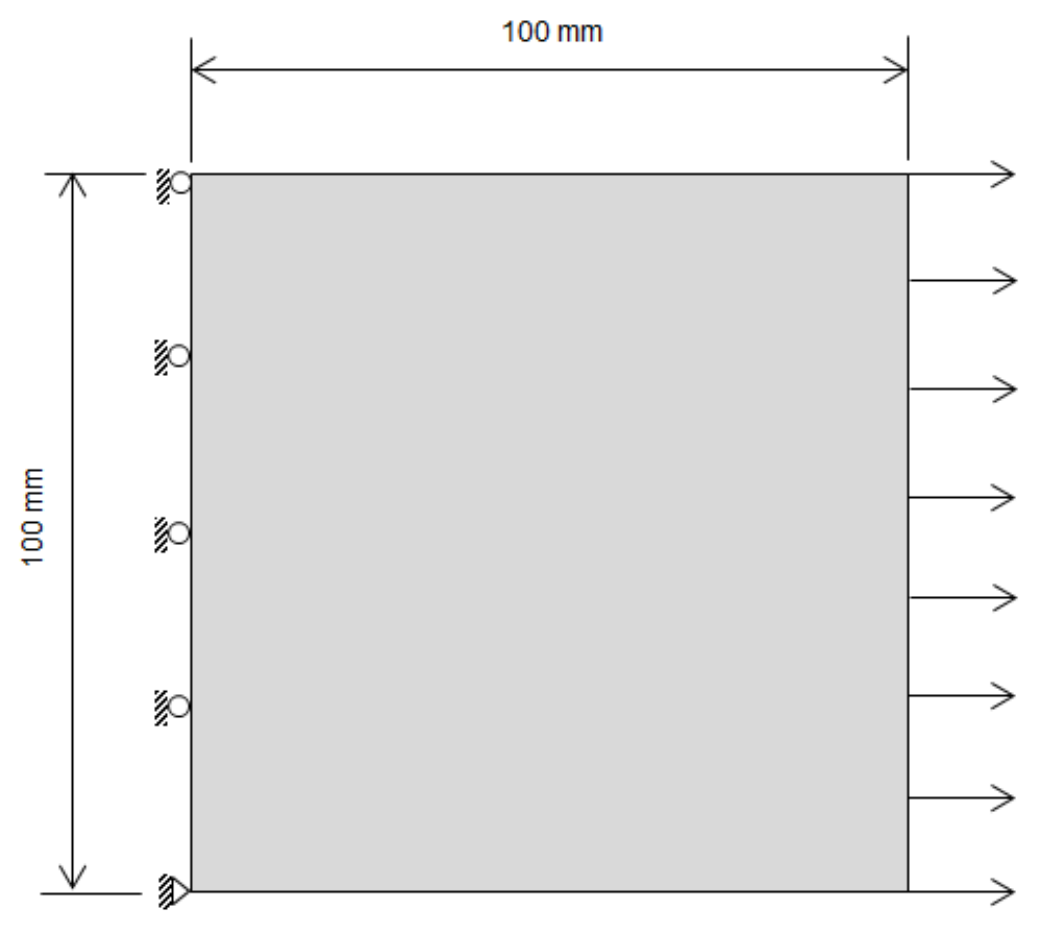

Figure 4.1: Geometry, boundary and loading condition of numerical model

the capsule shell made of urea formaldehyde are taken from experimental results and a perfect bond with its surrounding is assumed [Keller and Sottos, 2006; Wang et al., 2013]. The fracture properties of the capsules is based on recent experimental works [Gilabert et al., 2015; Hilloulin et al., 2015; Wang and Jivkov, 2015]. Due to the lack of experimental data, the shear fracture properties were assumed equal to the normal ones.

\subsection{Study of mesh size}

The models with different volume fraction of capsules ranging from $1.57 \%$ to $9.42 \%$ are created. Since it is well known that the results of cohesive elements are quite sensitive with respect to the underlying discretization, a mesh study was conducted. The results of four different realizations with the same element length of mesh $(\mathrm{Le}=1 \mathrm{~mm})$ as shown in Fig. 4.2 are presented. Four different 


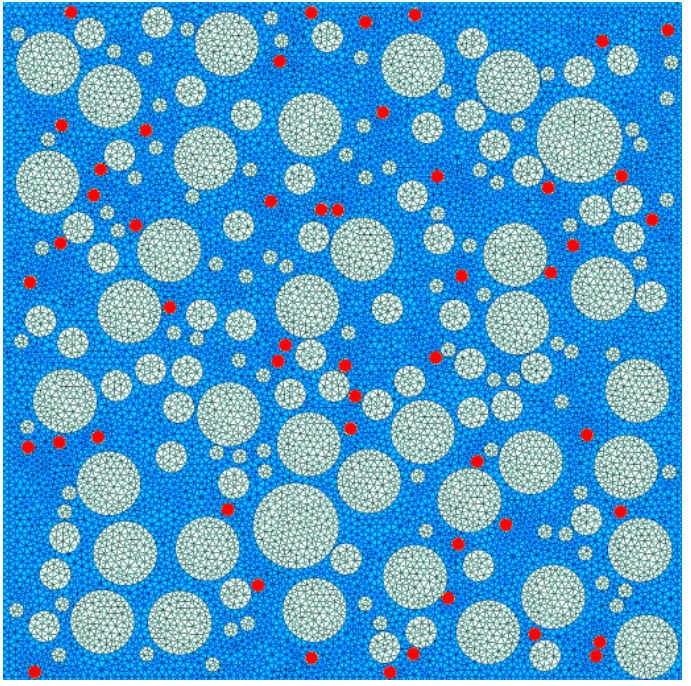

(a)

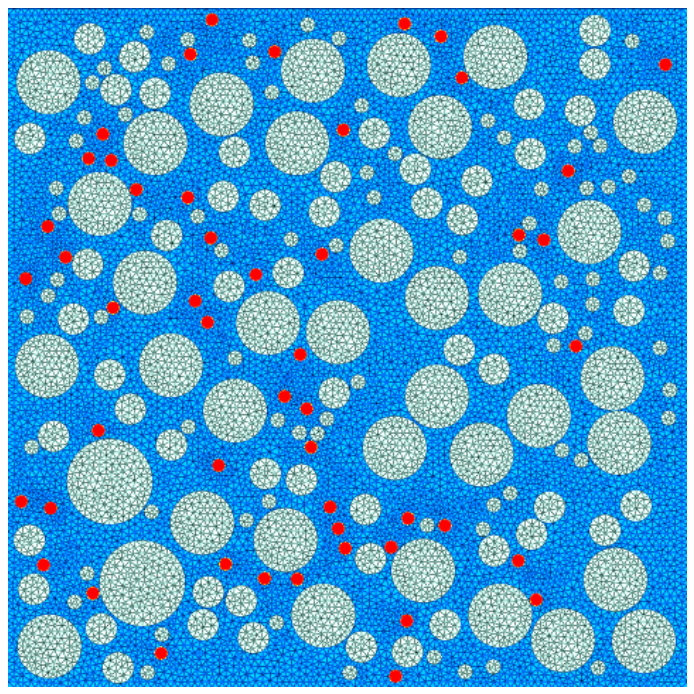

(c)

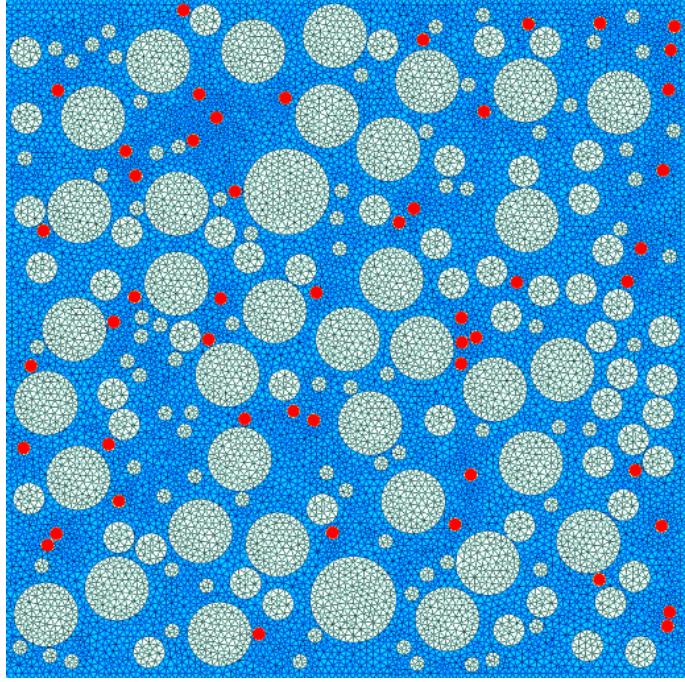

(b)

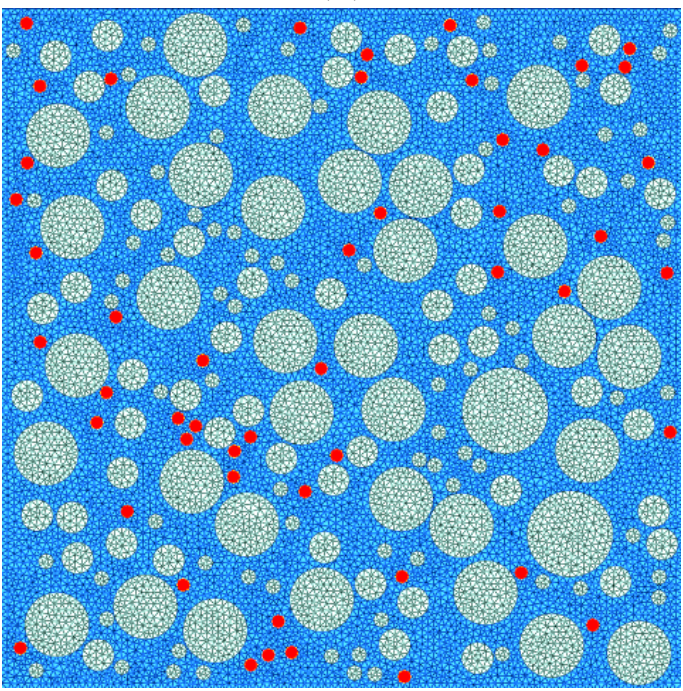

(d)

Figure 4.2: Different realization with the same mesh density $(\mathrm{Le}=1 \mathrm{~mm})$ at consistent volume fraction of aggregates $40 \%$ and capsules $1.57 \%$ 


\section{2D Numerical Simulations Under Uniaxial Tension}

Table 4.1: Material properties

\begin{tabular}{lcccccc}
\hline Model & $\begin{array}{c}\text { Young's } \\
\text { modulus E } \\
(\mathrm{MPa})\end{array}$ & $\begin{array}{c}\text { Poisson's } \\
\text { ratio }\end{array}$ & $\begin{array}{c}\text { Density } \\
\left(10^{-9}\right. \\
\left.\text { tonne } / \mathrm{mm}^{3}\right)\end{array}$ & $\begin{array}{c}\text { Elastic } \\
\text { stiffness } \\
k_{n}(\mathrm{MPa} / \mathrm{mm})\end{array}$ & $\begin{array}{c}\text { Cohesive } \\
\text { strength } \\
t_{n}(\mathrm{MPa})\end{array}$ & $\begin{array}{c}\text { Fracture } \\
\text { energy } \\
G_{F}(\mathrm{~N} / \mathrm{mm})\end{array}$ \\
\hline Aggregate & 70000 & 0.2 & 2.5 & - & - & - \\
Mortar & 25000 & 0.2 & 2.2 & - & - & - \\
Capsule core & 1000 & 0.45 & 1.0 & - & - & - \\
Capsule shell & 3600 & 0.3 & 1.0 & - & - & - \\
CIE-AA & - & - & 2.5 & $10^{6}$ & - & - \\
CIE-AM & - & - & 2.2 & $10^{6}$ & 3 & 0.03 \\
CIE-MM & - & - & 2.2 & $10^{6}$ & 6 & 0.06 \\
CIE-CC & - & - & 1.0 & $10^{6}$ & - & - \\
CIE-CS & - & - & 1.0 & $10^{6}$ & 10 & 0.1 \\
CIE-SS & - & - & 1.0 & $10^{6}$ & 10 & 0.1 \\
CIE-SM & - & - & 1.0 & $10^{6}$ & 10 & 0.1 \\
\hline
\end{tabular}

realizations lead to different final crack paths. Cracks (white color) represented by cohesive interface elements with damage index $\mathrm{D} \geq 0.9(\mathrm{D}=1$ means complete failure) are shown magnifiied with a scaling factor of 10 for uniformly deformed meshes. As can be observed in Fig. 4.3(a), (b), and (c), these samples fail with a single main macrocrack with different location of failure, and the other sample fails with double main macrocracks (Fig. 4.3(d)). These results show the necessity to conduct statistical analysis with regard to different distribution of microstructures inside the mortar matrix.

In order to study the effect of mesh densities, three mesh sizes with element length Le $=1 \mathrm{~mm}, 0.75 \mathrm{~mm}$ and $0.5 \mathrm{~mm}$, respectively as shown in Fig. 4.4 are considered. The mesh 1 has 76,086 nodes, 25,362 solid elements and 37,843 cohesive elements. Mesh 2 has 122,790 nodes, 40,930 solid elements and 61,129 cohesive elements, and mesh 3 271,794 nodes, 90,598 solid elements and 135,497 cohesive elements. For each element length, 20 numerical samples with random distribution of circular aggregates and capsules with volume fraction $1.57 \%$ were generated and analyzed in order to ensure that the results were statistically converged.

Fig. 4.5 shows that the final crack paths of three different meshes density are quite similar. The mean stress-displacement $(\sigma-\mathrm{d})$ curves are compared for each element length. The stress is the total horizontal reaction forces of all nodes at 


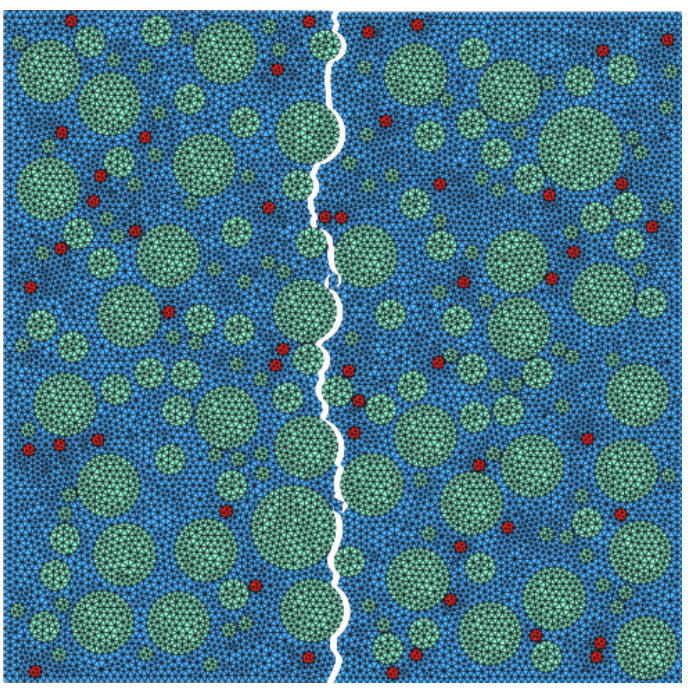

(a)

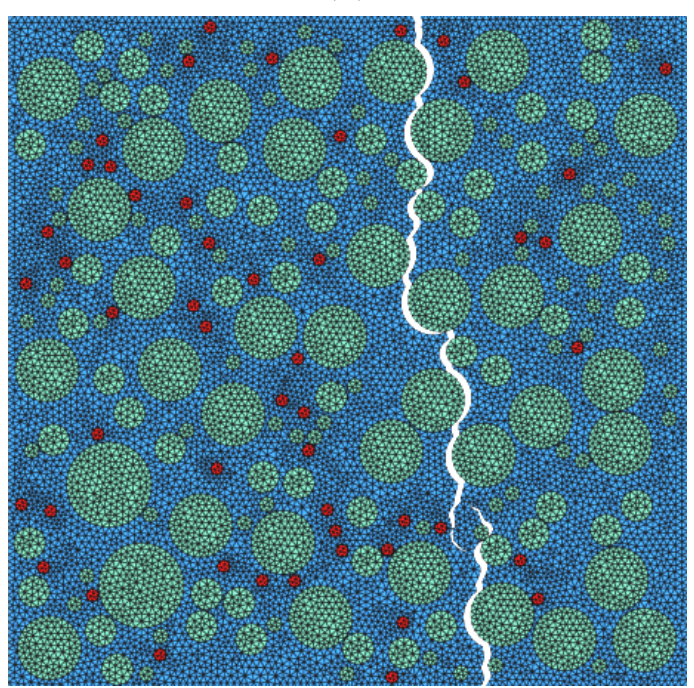

(c)

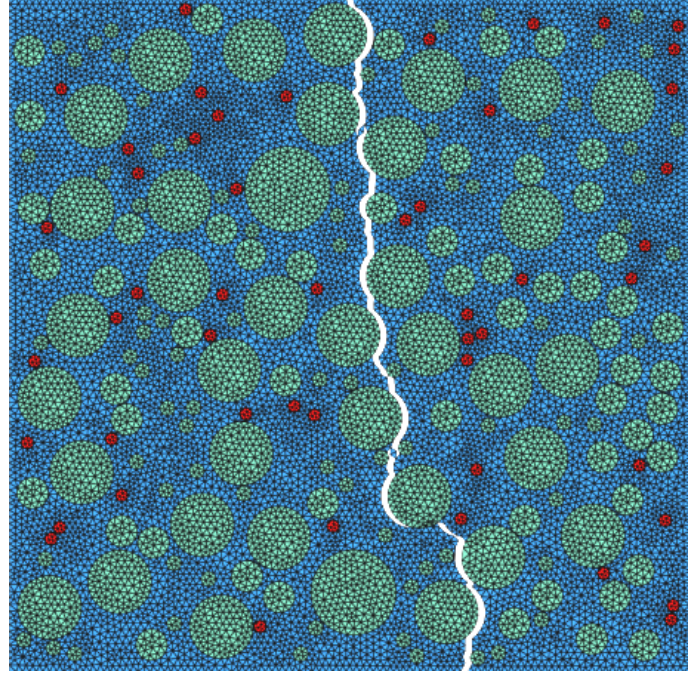

(b)

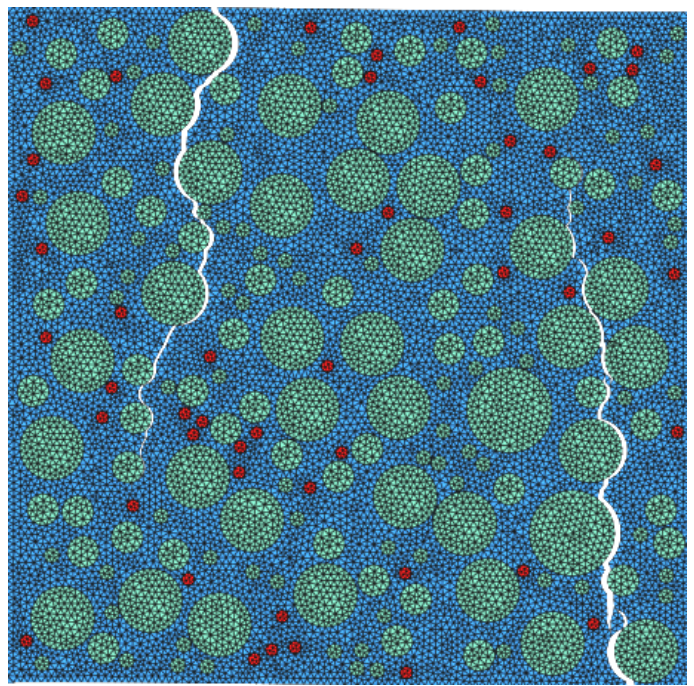

(d)

Figure 4.3: Final crack paths for different realization of mesostructures. (a) Realization 1, element length $=1 \mathrm{~mm}$, (b) Realization 2, element length $=1$ $\mathrm{mm}$, (c) Realization 3, element length $=1 \mathrm{~mm}$, (d) Realization 4, element length $=1 \mathrm{~mm}$. 


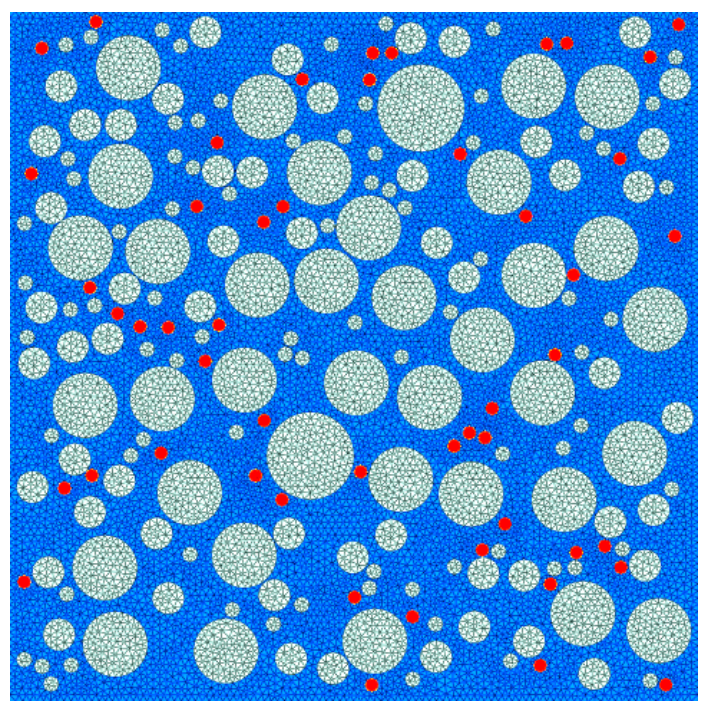

(a)

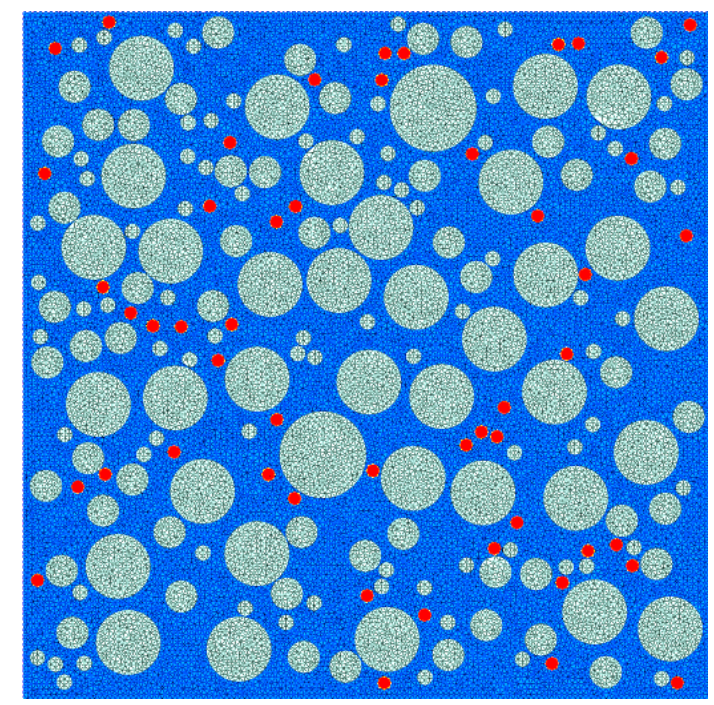

(b)

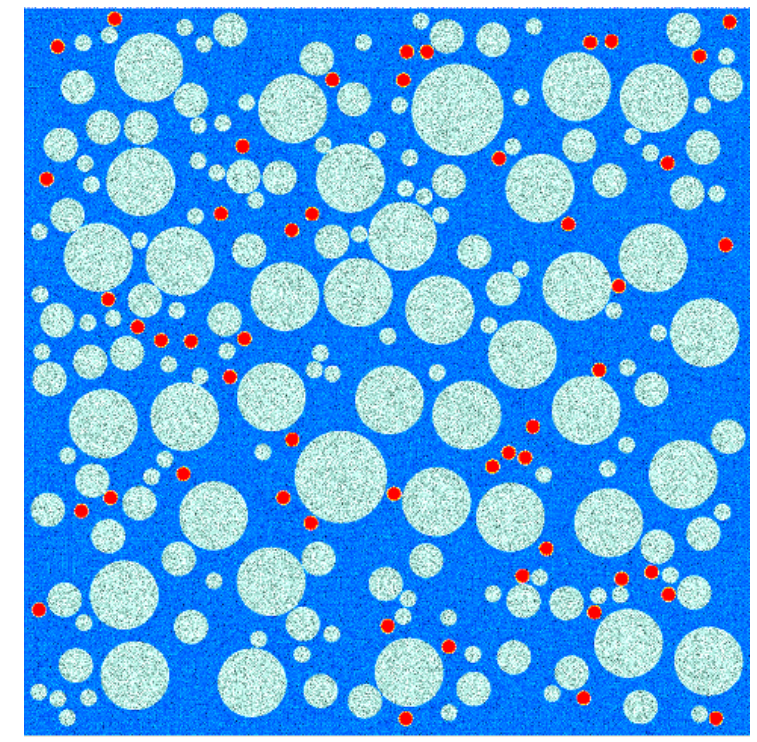

(c)

Figure 4.4: Three different meshes with different density. (a) Mesh 1 (Le $=1$ $\mathrm{mm})$. (b) Mesh $2(\mathrm{Le}=0.75 \mathrm{~mm})$. (c) Mesh $3(\mathrm{Le}=0.5 \mathrm{~mm})$. 
the left boundary divided by the cross section area of specimen. As can be seen in Fig. 4.6, the results are nearly identical. Therefore, the subsequently presented results only for simulations based on an element length of $1 \mathrm{~mm}$.

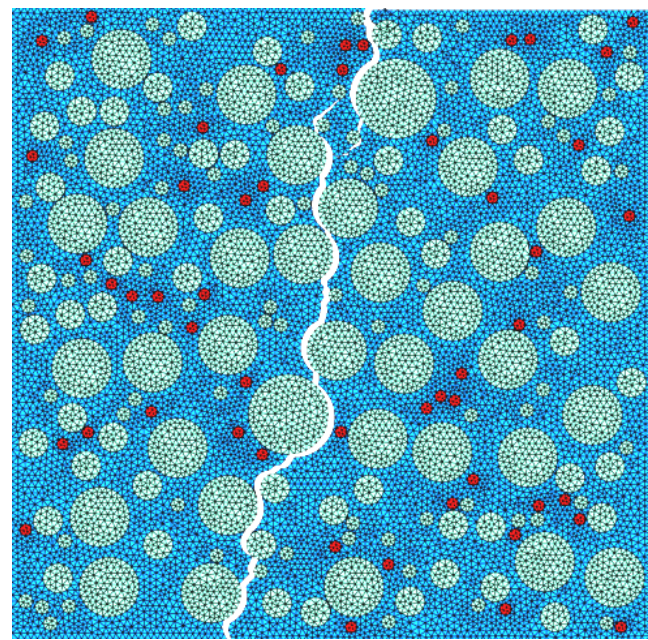

(a)

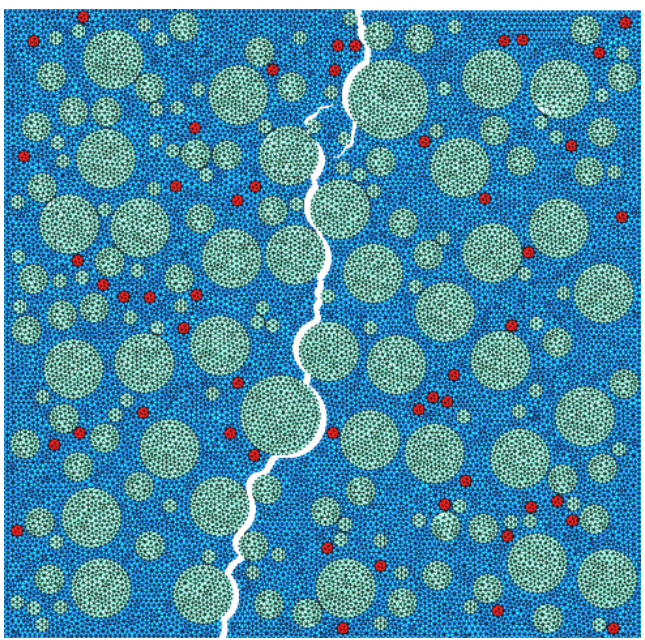

(b)

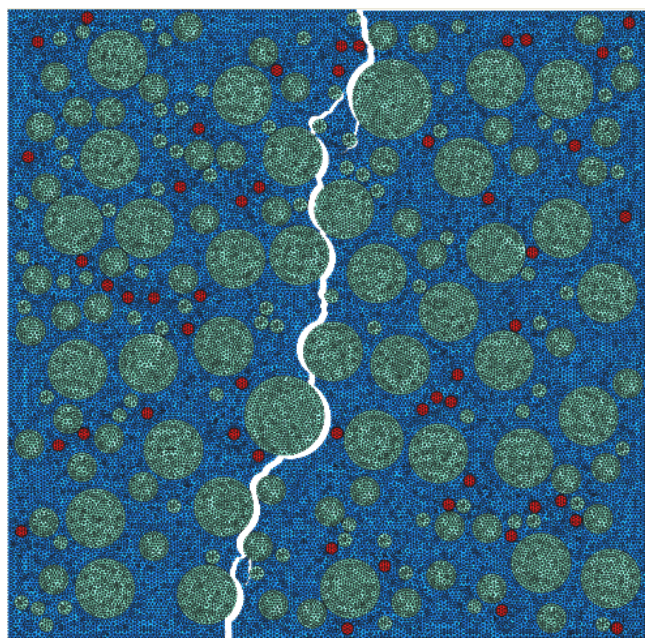

(c)

Figure 4.5: Final crack paths for different mesh density. (a) Mesh 1 (Le $=1 \mathrm{~mm})$. (b) Mesh $2(\mathrm{Le}=0.75 \mathrm{~mm})$. (c) Mesh $3(\mathrm{Le}=0.5 \mathrm{~mm})$. 


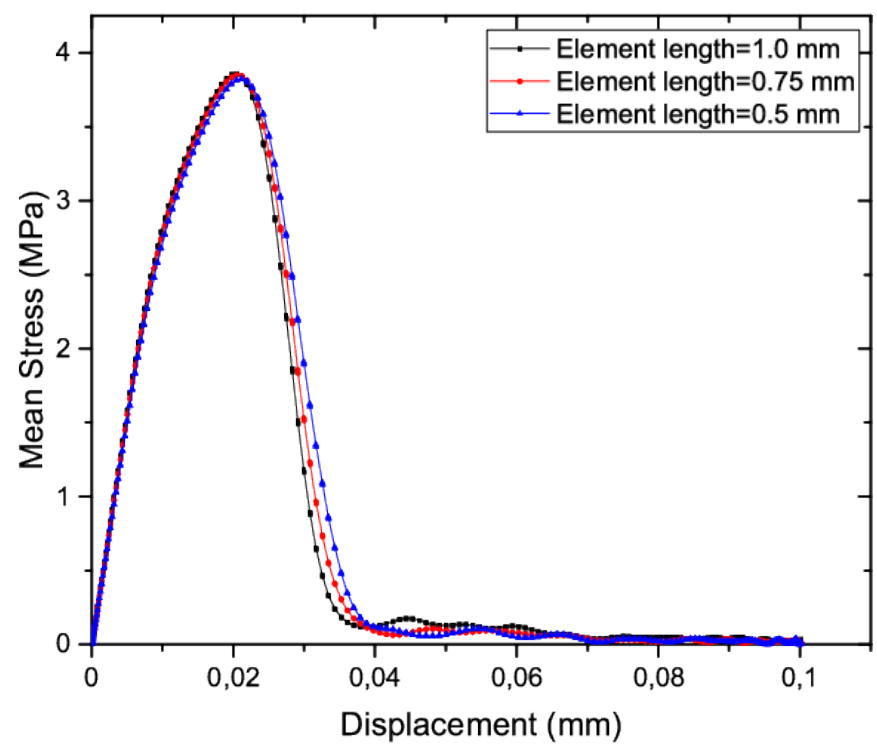

Figure 4.6: Mean stress-displacement curves for different meshes.

\subsection{Numerical results}

\subsubsection{Effect of capsules volume fraction}

The exemplary results of stress-displacement curves computed from 20 random samples with random distribution of aggregates and volume fraction of capsules $1.57 \%$ can be seen in Fig. 4.7. The mean curve and standard deviation of the peak stress are also shown. The predicted stress-displacement curves are identical for all random models with a clear peak followed by post-peak drop. The scatter curves obtained from simulations show the necessity of statistical analysis with random distribution of aggregates and capsules. In addition, all the statistical results from 20 random samples with different aggregate distributions were illustrated in Fig. 4.8. 


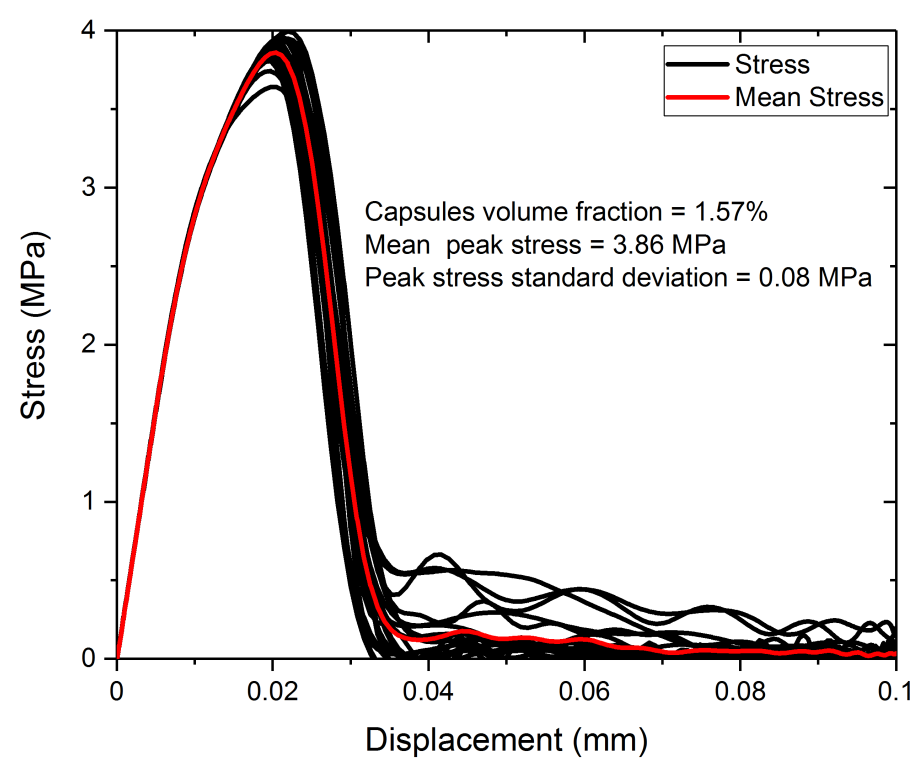

Figure 4.7: Stress-displacement curves from 20 random samples with volume fraction of capsules $1.57 \%$

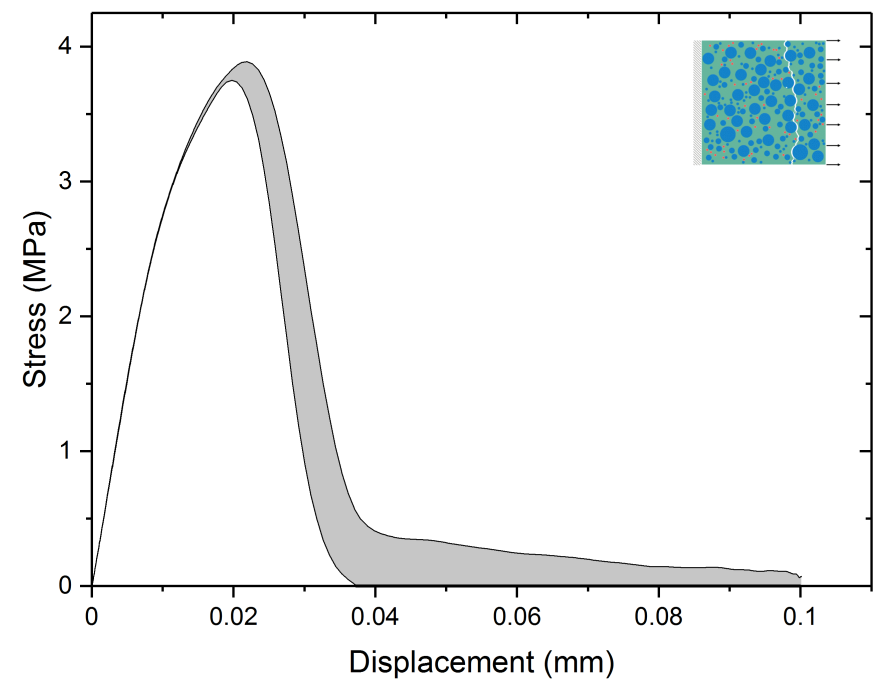

Figure 4.8: Statistical results of stress-displacement curves from 20 random samples 
The gray area along the curves highlighted standard deviation values from all model conditions. It can be observed in Fig. 4.8 that, the initial behavior is elastic and followed by a peak and then softening branch. The influence of capsules volume fraction at the pre-peak regime is not significant. However, from the peak and post-peak range, the influence can be observed clearly, which is caused by the variation of crack propagations.

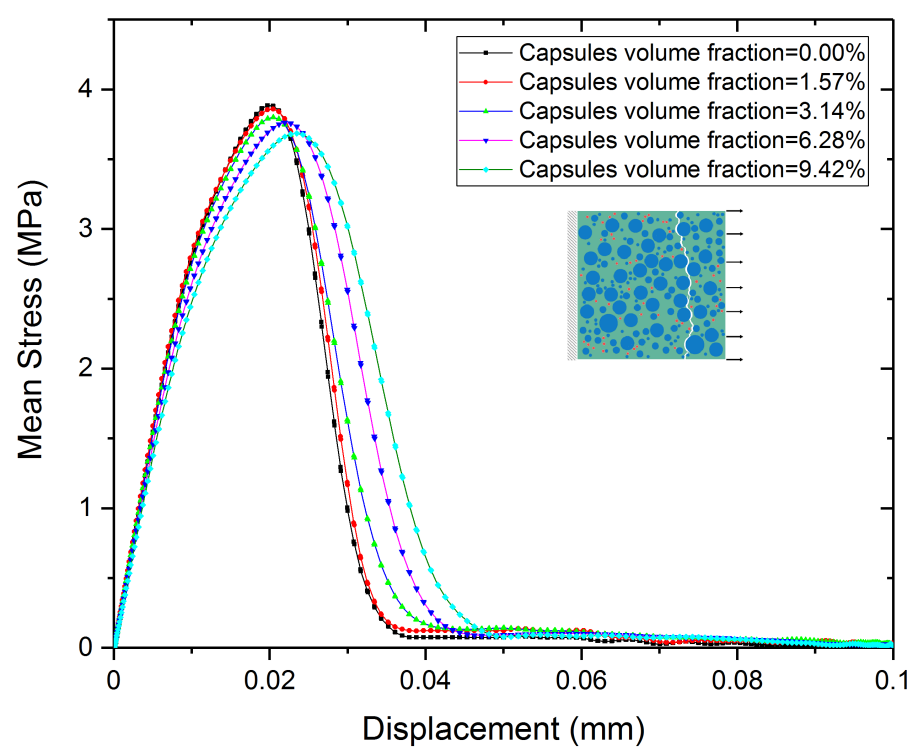

Figure 4.9: Comparison of mean stress-displacement curves in uniaxial tension.

Fig. 4.9 and Fig. 4.10 show the mean stress-displacement curves and corresponding peak stress, respectively for different capsules volume fraction ranging from $1.57 \%$ to $9.42 \%$. The original model which is without capsules has the highest mean stress around 3.88 MPa. The highest mean stress of self-healing concrete is obtained by the samples with volume fraction of capsules $1.57 \%$ around 3.87 $\mathrm{MPa}$ whereas the lowest one is obtained by volume fraction $9.42 \%$ with a mean peak stress around 3.6 MPa. 


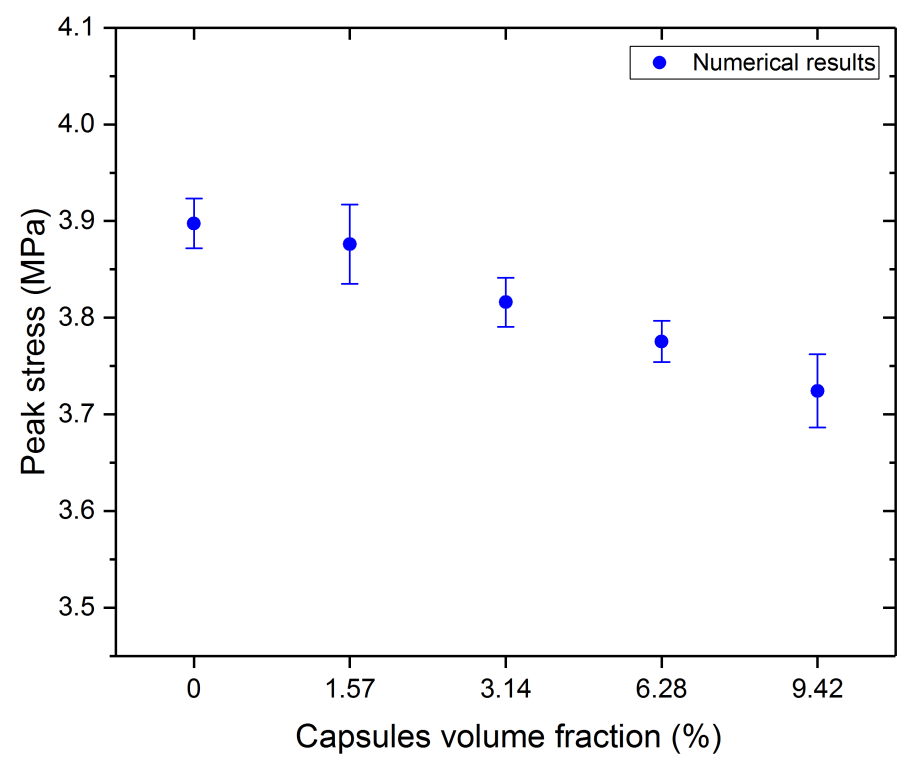

Figure 4.10: Effects of capsules volume fraction to the peak stress.

The initial elastic stiffness of the specimens decreases as more capsules are added. The higher the volume fraction of capsules embedded in the concrete, the lower the strength of self-healing concrete and the higher the fracture strain. An increased volume fraction of capsules from $1.57 \%$ to $9.42 \%$ reduces the strength by $6.97 \%(3.87-3.6 \mathrm{MPa})$. However, when the volume of the capsules is in the range of $0-2 \%$, the variation of volume fraction of capsules almost does not affect the strength.

The decrease of strength caused by the amount of capsules as can be observed in Fig. 4.9 and Fig. 4.10 is consistent with the recent experimental work conducted by Wang et al. [2014] who used micro-encapsulated bacterial spores, Kanellopoulos et al. [2016] employed pig-gelatine microcapsules carrying sodium silicate and Lv et al. [2016] using poly (phenol-formaldehyde-PF) microcapsules. In addition, the capsule-embedded models behave more ductile compared to the one without capsules (Fig. 4.9). This phenomenon is in good agreement with the experimental results reported by Lv et al. [2016]. 


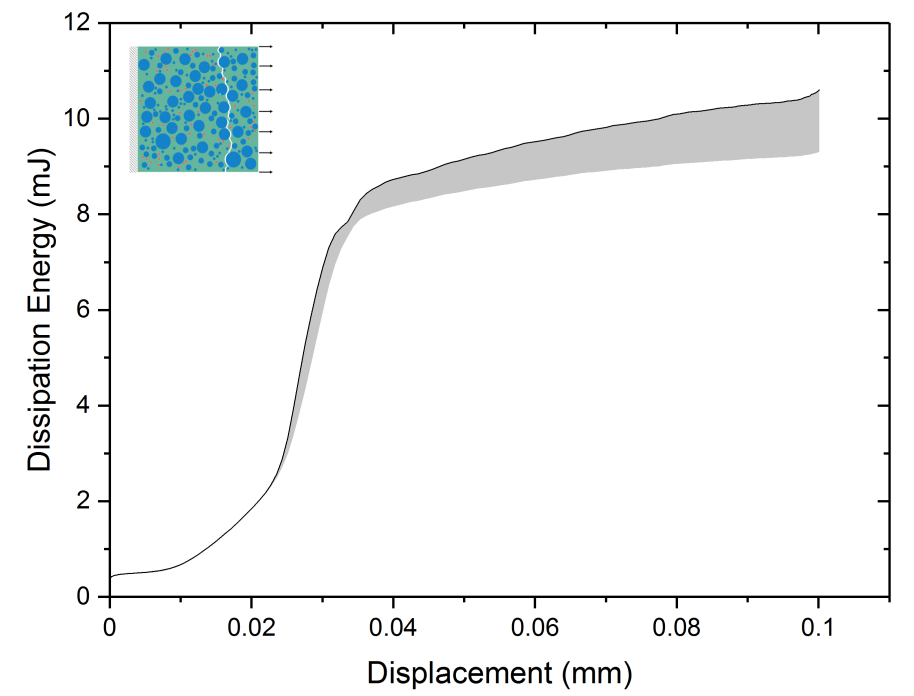

Figure 4.11: Statistical results of dissipation energy curves from 20 random samples.

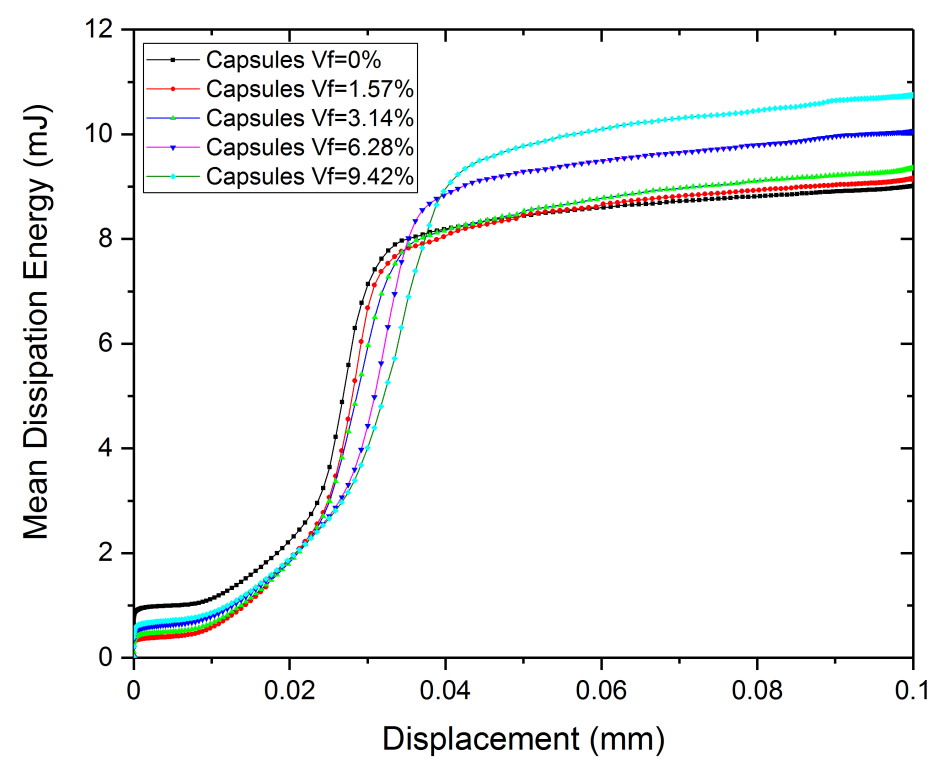

Figure 4.12: Mean energy-displacement curves with different volume fraction of capsules. 
Fig. 4.11 shows the statistic results of dissipated energy versus the displacement for 20 random samples with different capsules volume fractions. The dissipated energy is extracted from the cohesive zone model. The variation of standard deviation from different random models are shown with the gray area along the curves. It can be seen that the predicted dissipation energy-displacement curves are almost identical for all models in the pre-peak part, but deviate steadily from each other at the later stages.

Fig. 4.12 and Fig. 4.13 show the mean dissipation energy-displacement curves and the corresponding peak energy, respectively for different capsules volume fraction ranging from $1.57 \%$ to $9.42 \%$. It can be seen that the dissipating energy of self-healing concrete increases as more capsules added in the mortar matrix. Increasing the volume fraction of capsules from $1.57 \%$ to $9.42 \%$, the dissipation energy obtained of sample increases by $13.66 \%$ (9.29-10.76 mJ). This suggests a higher crack density. It is obvious that self-healing concrete materials need more energy to dissipate when more capsules added in the mortar matrix, since the density of micro-cracks is higher. However, the variation of dissipation energy of self-healing concrete does not change significantly when the volume fraction of capsules ranging from $0-2 \%$. 


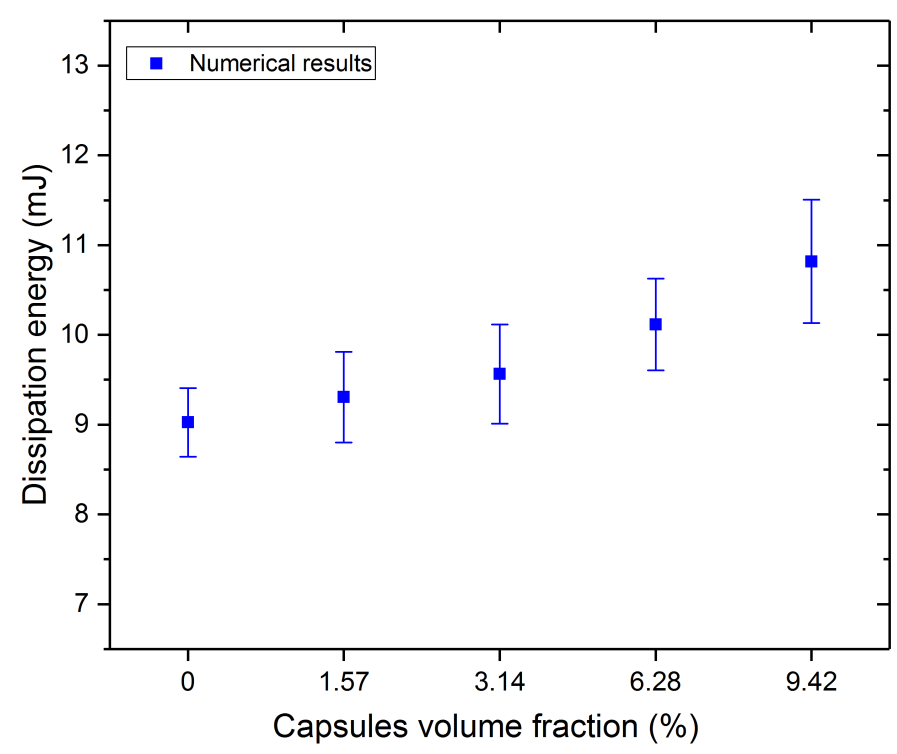

Figure 4.13: Effects of capsules volume fraction to the peak dissipation energy.

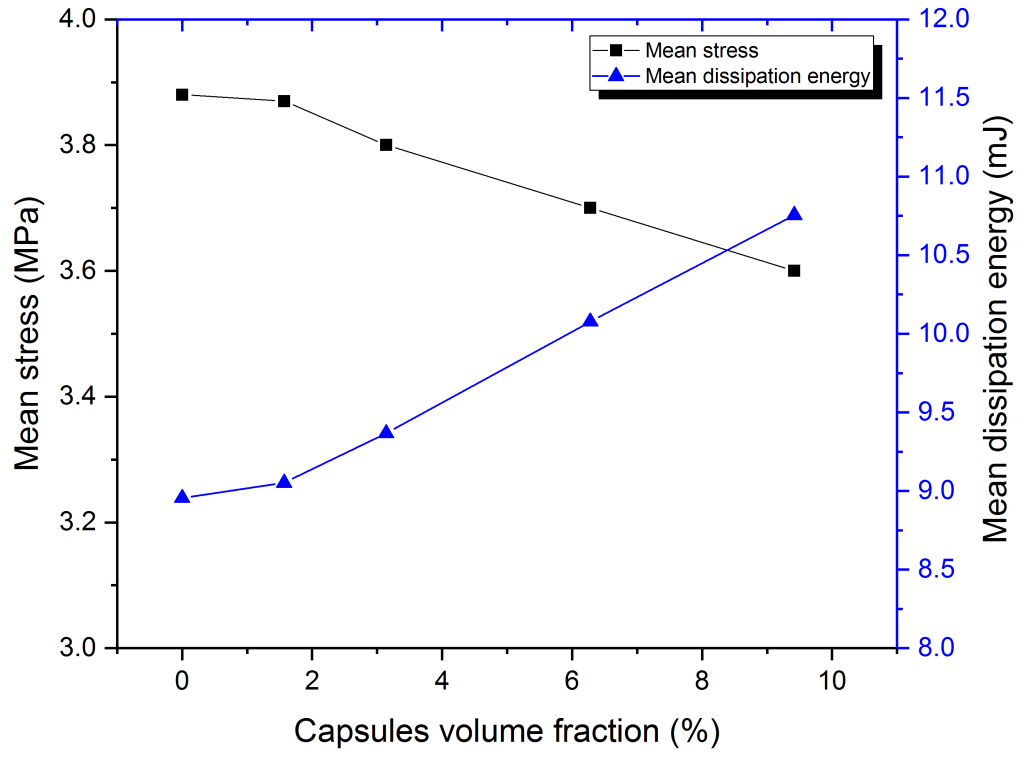

Figure 4.14: The relationship between mean stress, mean energy, and volume fraction of capsules. 
The relationship between mean stress, mean energy, and volume fraction of capsules can be seen in Fig. 4.14. It can be observed from Fig. 4.14 that adding more capsules to the mortar matrix will increase the dissipation energy in one hand, but in the other hand will decrease the peak stress of the self-healing concrete materials. As the interest is to investigate the breakages of capsules when a crack approaches them, we study the effect of volume fraction of capsules to the number of breakages. In this simulation, the fracture of the capsules are obtained from the fracture of capsule shells. As mentioned before, the subsequent healing process has not been modelled.

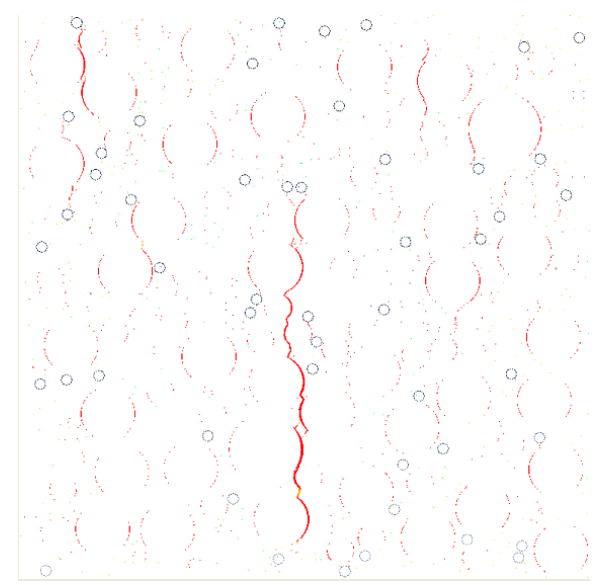

(a)

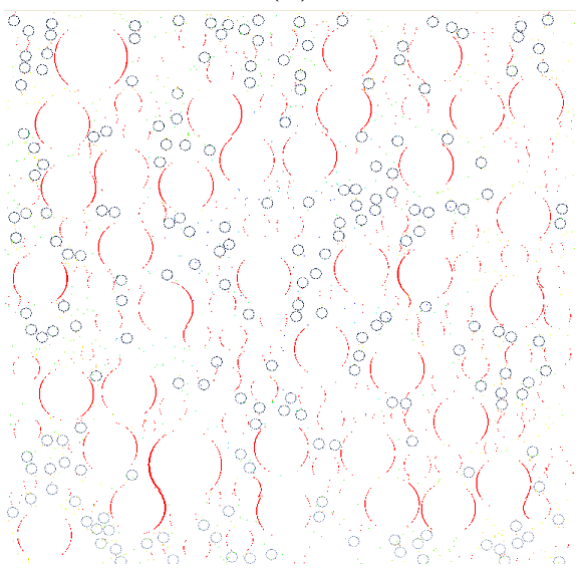

(c)

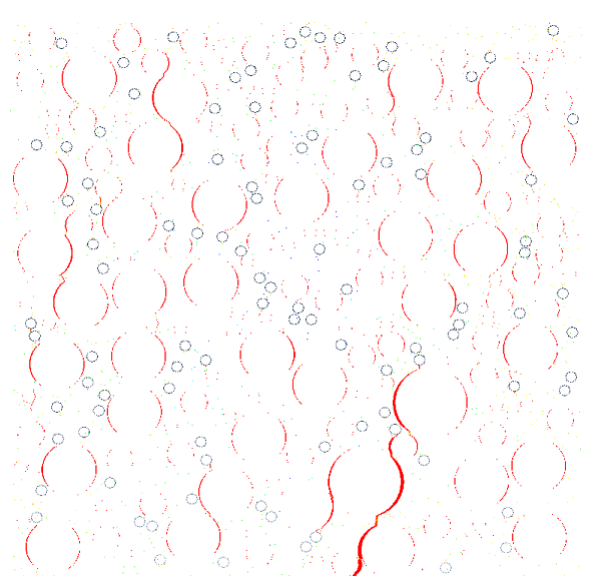

(b)

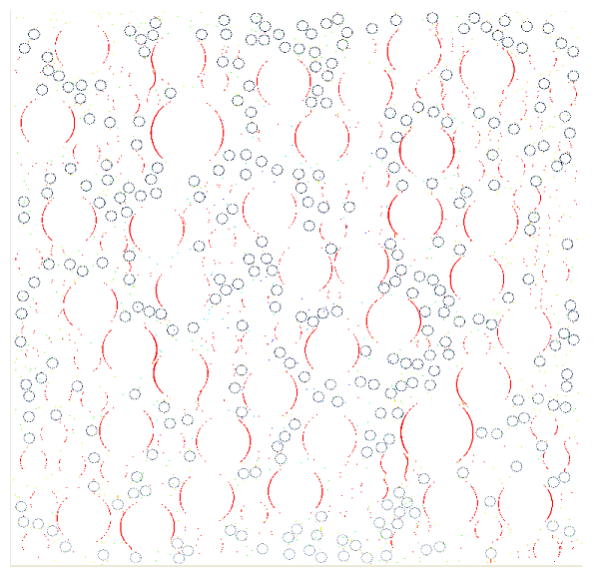

(d)

Figure 4.15: The generation of pre-peak microcracking for different volume fraction of capsules. (a) $\mathrm{Vf}=1.57 \%$ (b) $\mathrm{Vf}=3.14 \%$ (c) $\mathrm{Vf}=6.28 \%$ (d) $\mathrm{Vf}=9.42 \%$. 
Fig. 4.15 illustrates the generation of microcracks before peak load. A deformation magnification factor of 100 is used to clearly illustrate the detailed microcrack generation. The mean percentage of capsule fracture with respect to different volume fractions ranging from $1.57 \%$ to $9.42 \%$ can be observed in Fig. 4.16. The percentage of capsule fracture is obtained by dividing the total number of broken capsules with the total number of capsules embedded in the samples. It can be seen that the number of broken capsules increases as more capsules are added. An increase in the volume fraction of capsules from $1.57 \%$ to $9.42 \%$ increases the probability of fracture.

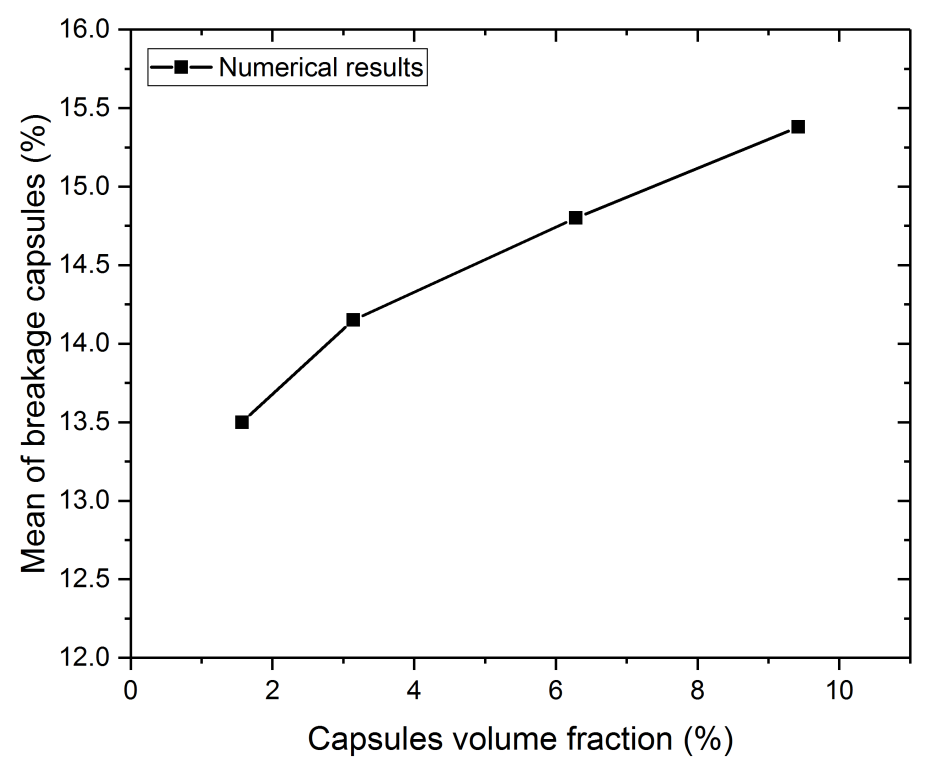

Figure 4.16: Effects of volume fraction of capsules to the percentage of capsules breakage.

The increasing number of broken capsules with respect to the amount of capsules (Fig. 4.16) is in good agreement with the experimental results that reported by Wang et al. [2013]. They showed that the recovery rate and healing efficiency of self-healing concrete are proportional to the amount of microcapsules. 


\subsubsection{Effect of capsule wall thickness}

In order to study the effect of the capsule's wall thickness to the probability of breakages, four different core-wall thickness ratio with 20 random samples each were tested as depicted in Fig. 4.17.

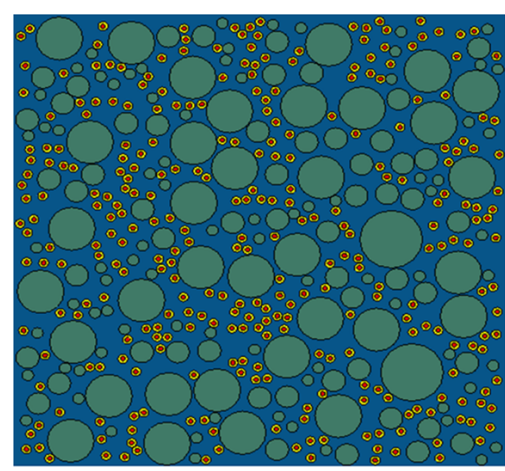

(a)

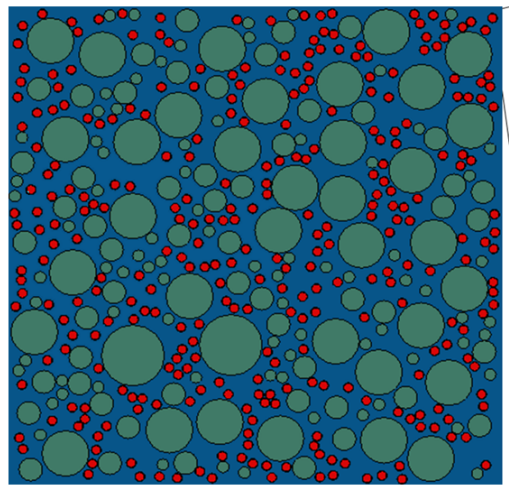

(c)
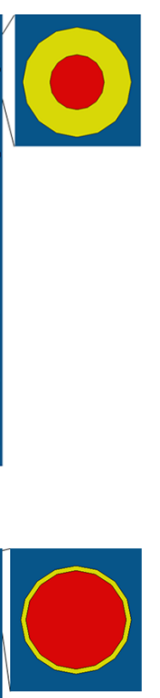
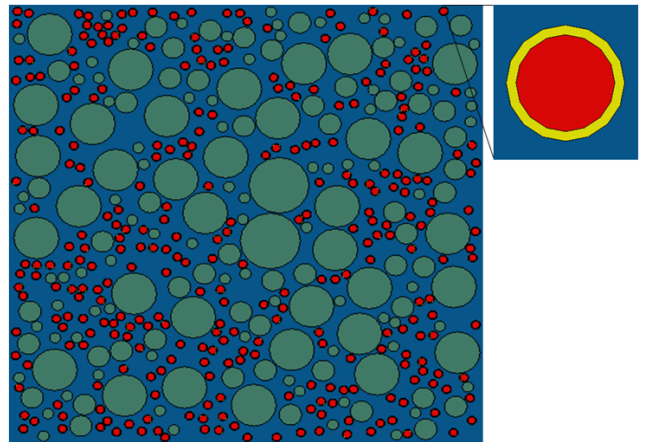

(b)
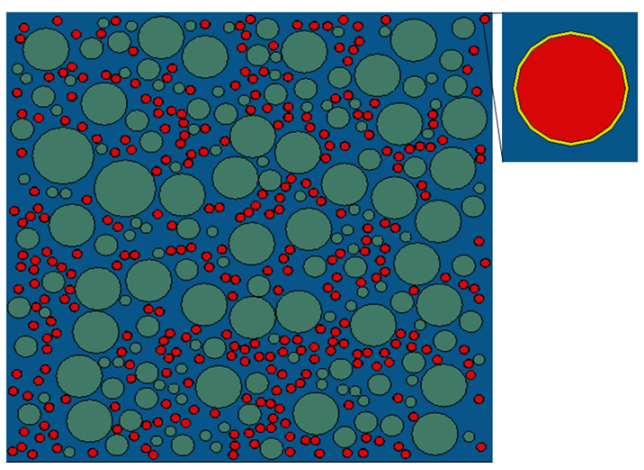

(d)

Figure 4.17: Microcapsule samples with different core-wall thickness ratio. (a) Ratio 1:1. (b) Ratio 5:1. (c) Ratio 10:1. (d) Ratio 15:1.

They show that capsules with the smallest core-shell ratio (1:1) have the largest thickness of the wall but the smallest volume of the healing agent and vice versa. All samples are based on the same volume fraction of aggregates (40\%) and capsules $(1.57 \%-9.42 \%)$. The geometry, boundary, and loading conditions of this simulation are identical to the ones explained in the previous section. Fig. 4.18 shows the exemplary mean stress-displacement curves for four different capsule core-wall thickness ratios with volume fraction of capsules $1.57 \%$. It can 
be observed that the peak stress and post-peak softening stages from all samples are identical. It is obvious that the capsule wall thickness has no significant effect on the strength of self-healing concrete.

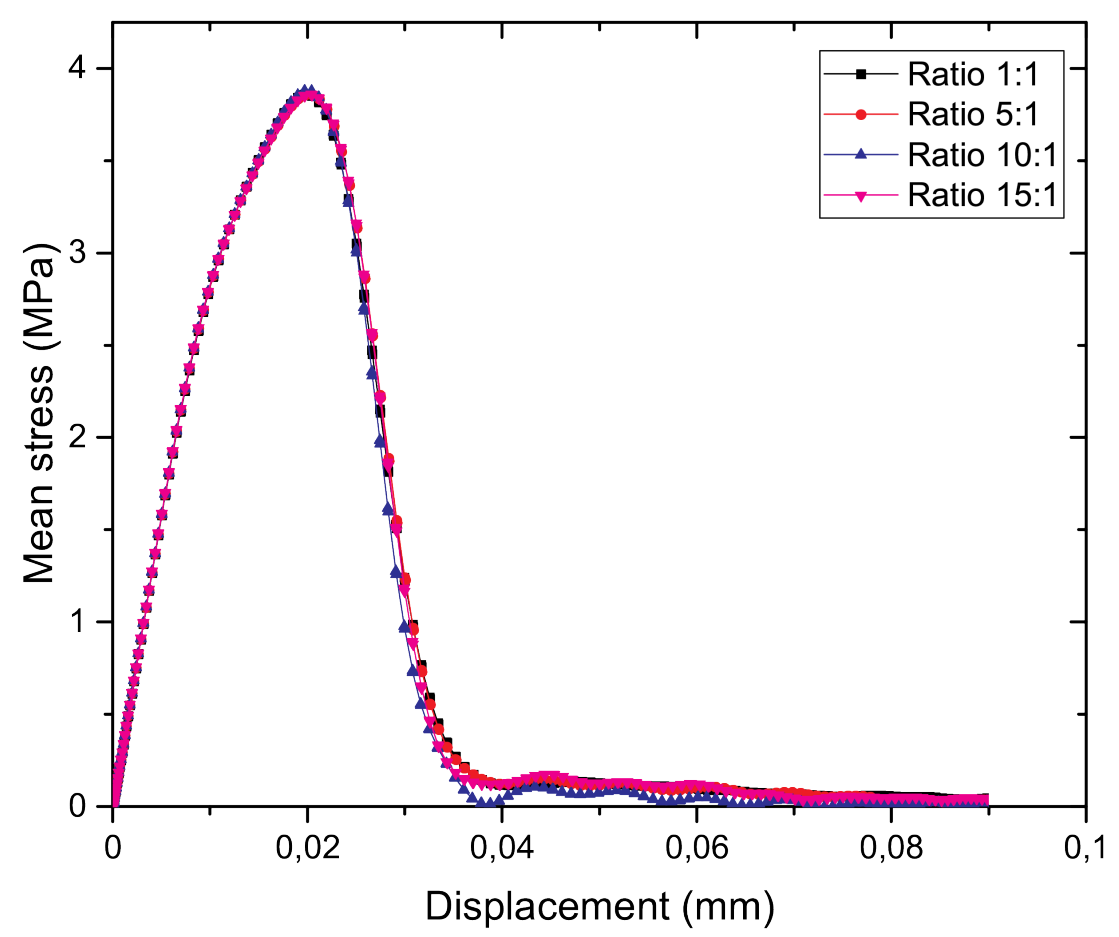

Figure 4.18: Mean stress-displacement curves from volume fraction of capsules $1.57 \%$ with different core-wall thickness ratio.

Fig. 4.19 shows the mean of breakage capsules from 20 random samples for different ratio of capsule core-wall thickness and volume fraction of capsules. The mean breakage value is calculated with respect to the total number of capsules and to the minimum volume fraction of capsules embedded in the mortar matrix (1.57\%). This simulation assumed that the capsule wall will break when the damage variable (SDEG) in the cohesive element reaches value between 0.9 and $1.0(0.9 \leq D \leq 1.0)$. 


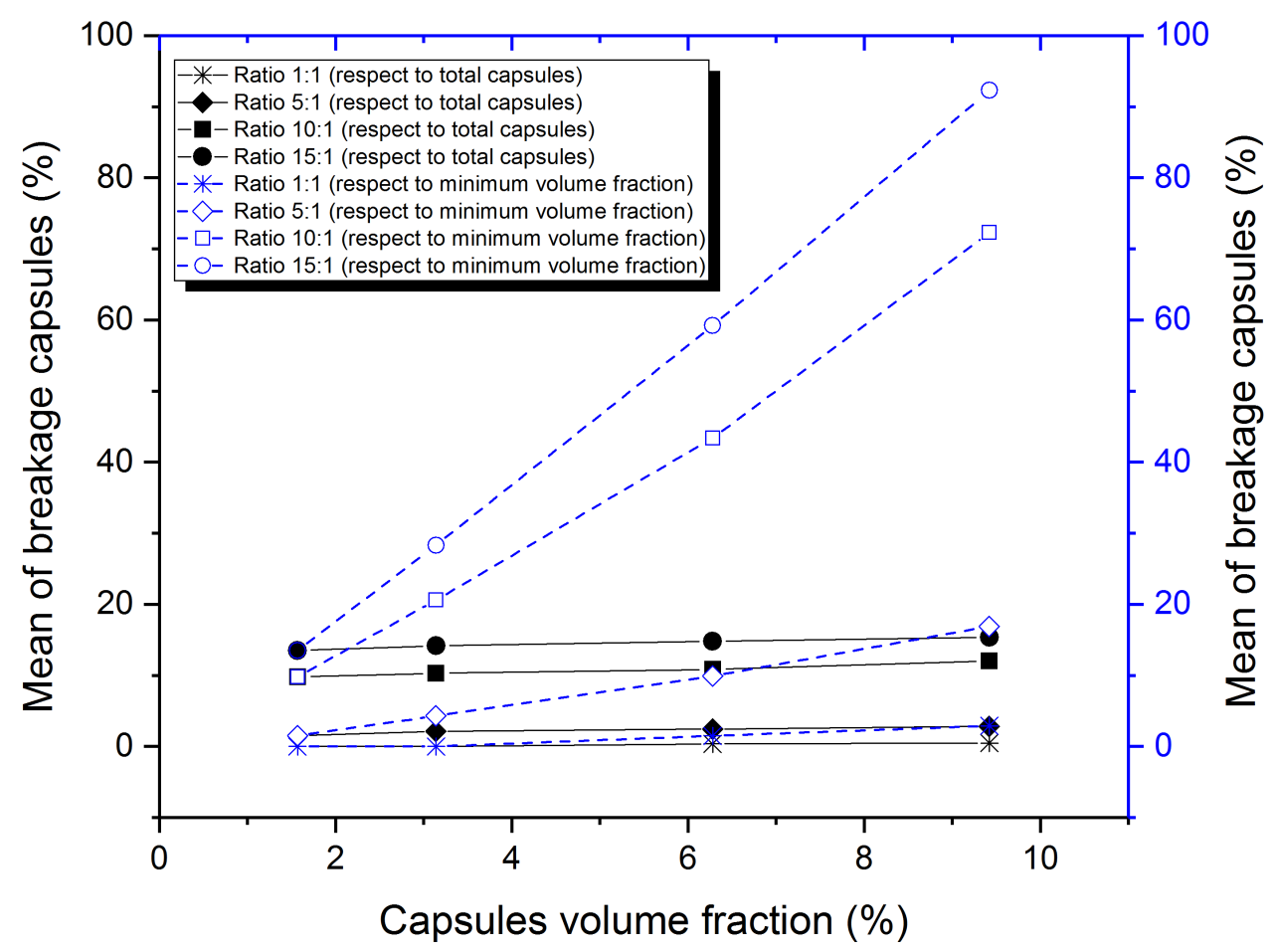

Figure 4.19: The mean of breakage capsules with corresponding core-wall thickness ratio and capsules volume fraction.

It is clear from Fig. 4.19 that although the ratio of the core-wall thickness of the capsule has no significant influence on the specimen strength, but has very significant effect on the fracture of the capsule shell. Increasing the core-shell thickness ratio led to increasing percentage of breakage from capsules and vice versa. It can be seen that the number of capsules with biggest ratio core-wall thickness (ratio 15:1) were broken more than the others. With respect to the minimum volume fraction of capsules, Ratio 15:1 and 10:1 increase noticeably when the volume fraction increases as well. Ratio 15:1 has the highest mean percentage of breakage (92.3\%) in all of volume fraction whereas the ratio $1: 1$ has the lowest one (2.9\%). Increasing volume fraction of capsules from $1.57 \%$ to $9.42 \%$ led to increase the percentage of breakage from $13.5 \%$ to $92.3 \%$ (Ratio $15: 1$ ), $9.8 \%$ to $72.3 \%$ (Ratio $10: 1$ ), $1.5 \%$ to $16.9 \%$ (Ratio $5: 1$ ), and $0 \%$ to $2.9 \%$ 
(Ratio 1:1). On the other hand with respect to the total number of capsules, there are no significant increases in percentage of breakage although volume fraction of capsules are added in the samples. The highest mean percentage of breakages is around $15 \%$. All results from this study are based only to the material properties found in the literatures, for example, the fracture properties of capsule shell (10 $\mathrm{MPa}$ ) used in this simulation are assumed larger than the mortar matrix (4 MPa). This conclusion only holds as long as that assumption is valid. In order to achieve an optimal healing efficiency of self-healing concrete compared to the traditional one, most of embedded capsules must be broken to restore the strength capacity. It requires further thorough investigation about the effects of mismatch properties between capsule and mortar matrix on the fracture of capsules. 


\section{Chapter 5}

\section{The Effect of Mismatch Properties on Fractured Capsule}

The focus of this section is to investigate the effect of mismatch properties between capsule and its surrounding matrix on fracture probability. The objective of study is not only to investigate the effect of mismatch in elastic and fracture properties but also to study the effect of capsule wall thickness on breakage of capsule. The strength of particles-dispersed type materials depends on bonding behavior between particle and matrix. The lower strength of bonding led to decreasing material capacity [Fu et al., 2008] and enhancement of fracture toughness from material might not be achieved. Since the weak interfacial zone would trigger debonding phenomena instead of fracture in the particle as can be observed in some experimental works [Rao et al., 1992; Sun and Yeomans, 1996]. Whereas in some special cases, where matrix and particle are brittle, weak interfacial bonding is more favored in facilitating effective mechanism for energy to dissipate [Yang and Jeng, 1991]. The advantage of dispersing particles inside matrix to elevate life-cycle capacity against failure has been adopted by some researchers in capsules-based self-healing concrete [Dong et al., 2015; Maes et al., 2014]. The capsules containing healing fluid are dispersed in mortar matrix during concrete mixing. Upon loading, an appearing crack propagates toward the capsule and break it then activating healing mechanism in the area of damage. 


\subsection{Model parameters}

The 2D rectangular concrete specimens with a circular capsule placed in the center were modeled. The uniaxial tension loadings are applied at the top of specimens. The specimen dimensions are $50 \mathrm{~mm}$ x $25 \mathrm{~mm}$ and the fixed capsule's diameter of $2 \mathrm{~mm}$ is used. The ratio of core-shell thickness 1:1 is considered in this preliminary simulation to ensure the resistant ability of capsule's wall for fracture during mixing process [Hilloulin et al., 2015].

For simplicity, the capsule core in this study assumed to be solid elements. To simulate starting point of propagation crack, an edge-crack is modeled at the left side of specimen with fixed length $4 \mathrm{~mm}$. The horizontal distance between the crack tip and the capsule is chosen large enough to ensure the influence of the particle on the crack driving force is suitably neglected. The geometry of model, boundary, and loading conditions are illustrated in Fig. 5.1. Vertical displacements were prescribed on the top of specimen. The Abaqus/Explicit with displacement-controlled algorithm was used to simulate the model with loading time $t=0.005 \mathrm{~s}$, and all simulations were finished at a displacement $d=0.1 \mathrm{~mm}$. 


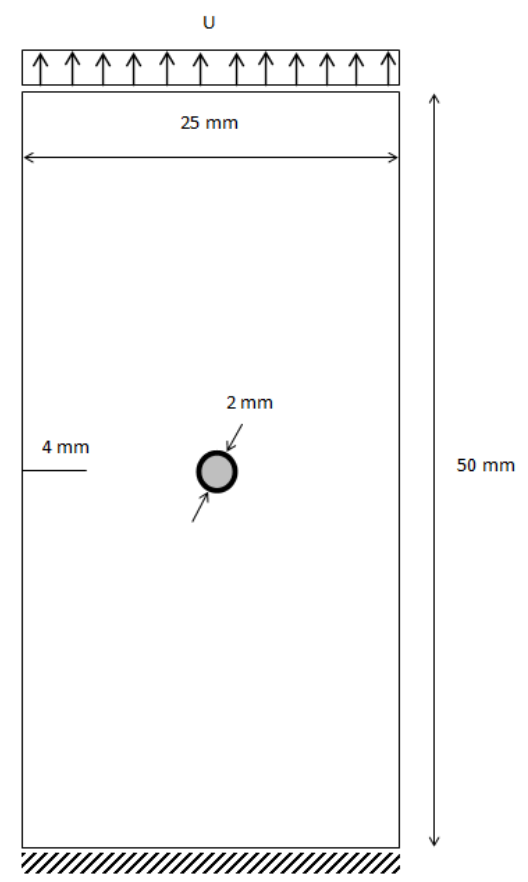

Figure 5.1: Geometry of the model, loading, and boundary conditions

The cohesive zone, which is represented by zero thickness cohesive element $\mathrm{COH} 2 \mathrm{D} 4$ in Abaqus, is defined by the constitutive behavior of traction-separation laws as described in the previous section. It illustrates the interaction between a cracked surface and the constitutive relation. The bilinear cohesive law between traction and its corresponding separation displacement on the cohesive surface is adopted in this study. It requires at least two parameters, such as the fracture energy and the cohesive strength.

\subsection{Mesh discretization}

All samples in this simulation are initially meshed with 3-node solid elements (CPS3). The mesh with element length of $1 \mathrm{~mm}$ is used in this study as illustrated in Fig. 5.2(a). The 4-node zero thickness cohesive elements are inserted into initial element meshes to represent potential cracks, such as inside the mortar matrix $\left(\mathrm{COH}_{\mathrm{MM}}\right)$, inside capsule shell $\left(\mathrm{COH}_{\mathrm{SS}}\right)$, between capsule core and capsule shell $\left(\mathrm{COH}_{\mathrm{CS}}\right)$, and between capsule shell and mortar matrix $\left(\mathrm{COH}_{\mathrm{SM}}\right)$. Since the 
flow process of healing agent into the crack area is not addressed in this study, the capsule core in this simulation is assumed to be solid and cracks are not allowed to initiate in the capsule core. The Python script combined with Abaqus batch processing technique is used to generate duplicate nodes and insert cohesive element automatically at each element interface. The typical discretization of the model after insertion of cohesive elements around the sample can be observed in Fig. 5.2(b).

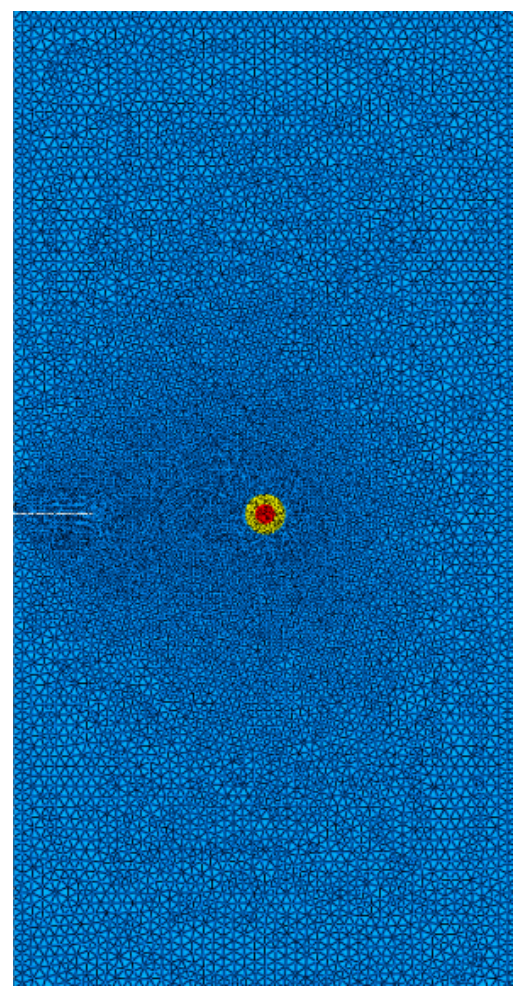

(a)

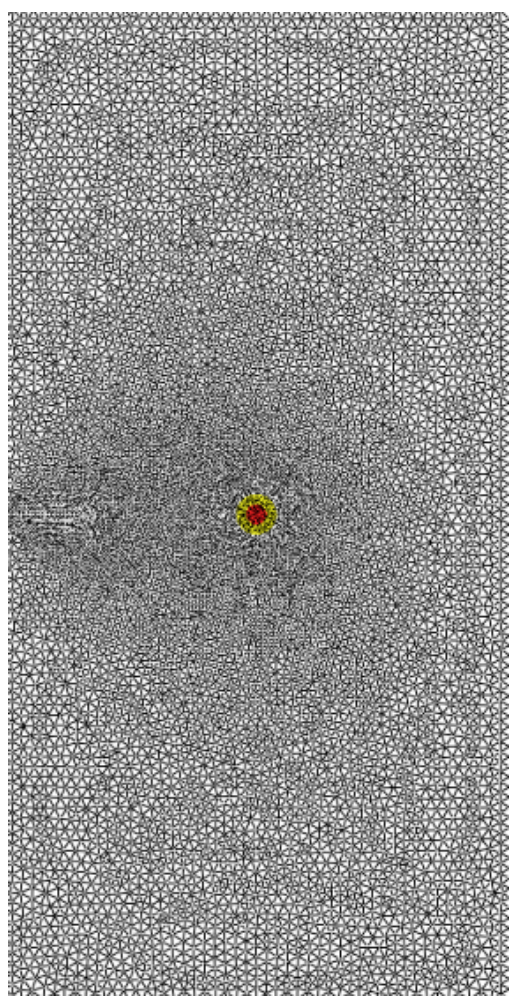

(b)

Figure 5.2: Mesh discretization of the model. (a) Initial mesh. (b) Inserted cohesive elements around the model.

\subsection{Parametric studies}

In capsule-based self-healing material, the fracture of capsule is highly expected. Once the capsule is fractured, it will release the healing agent to the area of damage and sealed the crack. The main objective of the present analysis is to study 
the effect of mismatch in elastic and fracture properties on the crack propagation. To do this, the extensive parametric studies are carried out considering that the elastic and fracture properties of capsule are varied relative to the fracture properties of the mortar matrix, such as the elastic modulus, $E_{c} / E_{m}$, the fracture strength, $t_{c} / t_{m}$, and the fracture energy, $G_{c} / G_{m}$. All values of parametric simulations and the range considered in the present study are summarized in Table 5.1. The subscript $m$ and $c$ indicates for mortar matrix and capsule, respectively. The quantity ratio given by $E_{c}$ to $E_{m}, t_{c}$ to $t_{m}$, and $G_{c}$ to $G_{m}$ are hereafter referred to as elastic ratio, strength ratio, and toughness ratio, respectively. For the sake of simplicity, the Poisson's ratios of the mortar matrix and the capsule, $\nu_{m}$ and $\nu_{c}$, are kept constant in all simulations, with $\nu_{m}=0.2$ and $\nu_{c}=0.3$ as found in Keller and Sottos [2006]. The bonding between the capsule and the mortar matrix is assumed to be perfect, with applying the large value of fracture strength and fracture energy into the interfacial zone, such that the separation at the capsule-mortar matrix interface is restrained. The shell thickness ratio of $1: 1$ is used in this preliminary simulations to investigate the effects of mismatch fracture properties on fractured capsule with the variation of elastic ratios between the mortar matrix and the capsule.

Table 5.1: Ratio of material properties used in this simulation

\begin{tabular}{lcc}
\hline Elastic modulus, & $\left(E_{c} / E_{m}=2,1,1 / 7\right)$ & $\nu_{m}=0.2$ \\
Fracture strength, & $\left(0.1<t_{c} / t_{m}<10\right)$ & $\nu_{c}=0.3$ \\
Fracture toughness, & $\left(0.01<G_{c} / G_{m}<50\right)$ & \\
\hline
\end{tabular}

\subsection{Numerical results}

\subsubsection{Mismatch of elastic and fracture properties}

The present simulations to study the effect of mismatch elastic and fracture properties on the crack propagation are reported in this section. The first part of this section provides an overview of parametric study with highlighting different parameters used and also describes the particular crack patterns. The results of the 


\section{The Effect of Mismatch Properties on Fractured Capsule}

parametric study will be summarized in the last section in term of fracture maps to distinguish the mechanism of fracture.

\subsubsection{Fracture patterns}

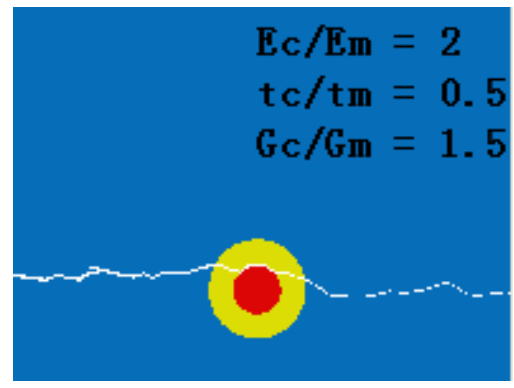

(a)

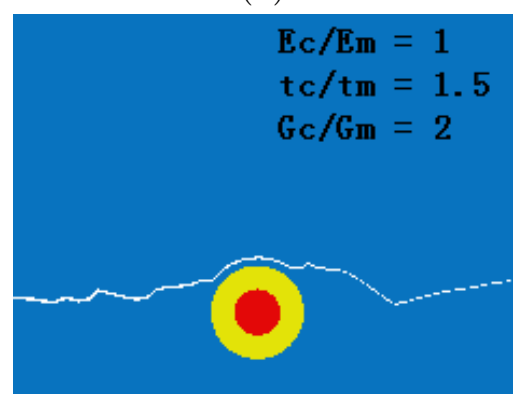

(c)

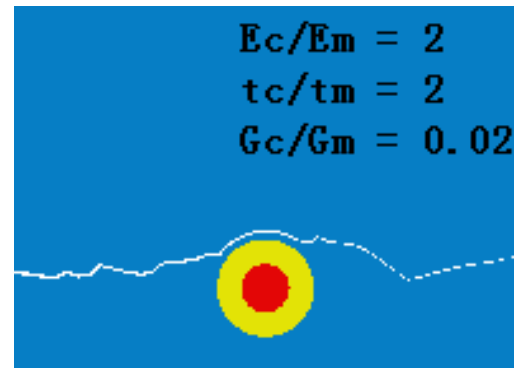

(e)

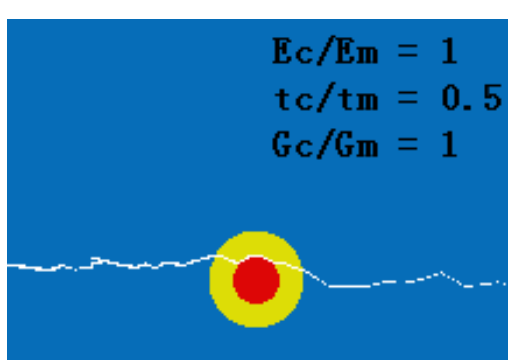

(b)

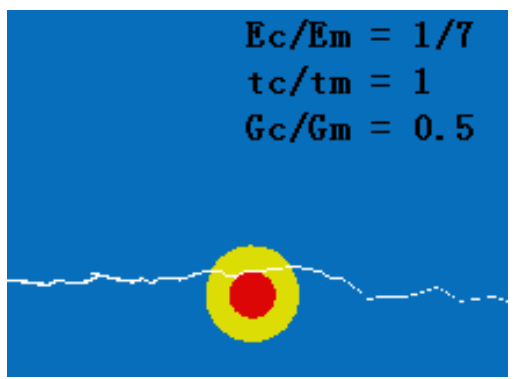

(d)

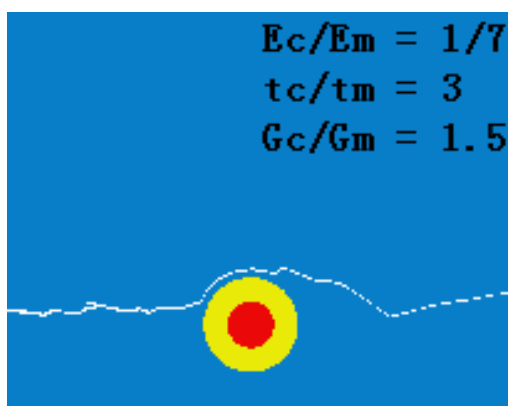

(f)

Figure 5.3: Failure path of an incoming crack through capsule based on mismatch in elastic and fracture properties.

The location of incoming crack is assumed to be located directly in front of the capsule, so there is no crack offset assumed in all simulations. The relative elastic and fracture properties ratio between the matrix and the capsule define 


\section{The Effect of Mismatch Properties on Fractured Capsule}

the actual path of crack as it approaches into the capsule as shown in Fig. 5.3. Figures with $E_{c} / E_{m}=2$ correspond to the stiffer capsule (Fig. 5.3(a) and (e)), whereas figures with $E_{c} / E_{m}=1 / 7$ correspond to softer capsule (Fig. 5.3(d) and (f)). The other relative ratio of fracture strength and toughness are varied correspondingly as described in the Figures. The capsule is initially undamaged and has a good bonding with the surrounding mortar matrix. All crack patterns illustrated in Fig. 5.3 are initially the same until it reaches a distance around to the diameter of the capsule. It can be seen that capsule fracture is observed in three cases ( $a, b$ and $d$ ) whereas crack deflection occurs in other cases (c, e and $\mathrm{f}$ ). Using the same elastic ratio between the capsule and the mortar matrix, the incoming crack deflects along the capsule when the strength of the capsule is larger than the strength of the mortar matrix whereas the capsule fracture occurs when the strength of the capsule is less than the strength of the mortar matrix as shown in Fig. 5.3(f) and (d), Fig. 5.3(c) and (b), and Fig. 5.3(e) and (a), respectively. Nonetheless, the mismatch in elastic properties between the capsule and the mortar matrix does not guarantee capsule fracture despite higher stiffness of the capsule.

\subsubsection{Fracture maps}

The effect of mismatch in elastic properties is investigated by considering three different elastic ratios, namely $E_{c} / E_{m}=2,1$ and $1 / 7$. In all simulations, the incoming crack has no initial offset from the center of the capsule (the crack lies directly in front of the center of the capsule). The results of these extensive simulations are summarized in the form of a fracture map, as illustrated in Fig. 5.4. The corresponding curve for each elastic properties ratios 2,1 and $1 / 7$, separates the areas of the fracture strength ratio $t_{c} / t_{m}$ and the fracture energy ratio $G_{c} / G_{m}$ for which the capsule would be either fractured or not because of an incoming crack, as shown in Fig. 5.4a, b and c, respectively. 


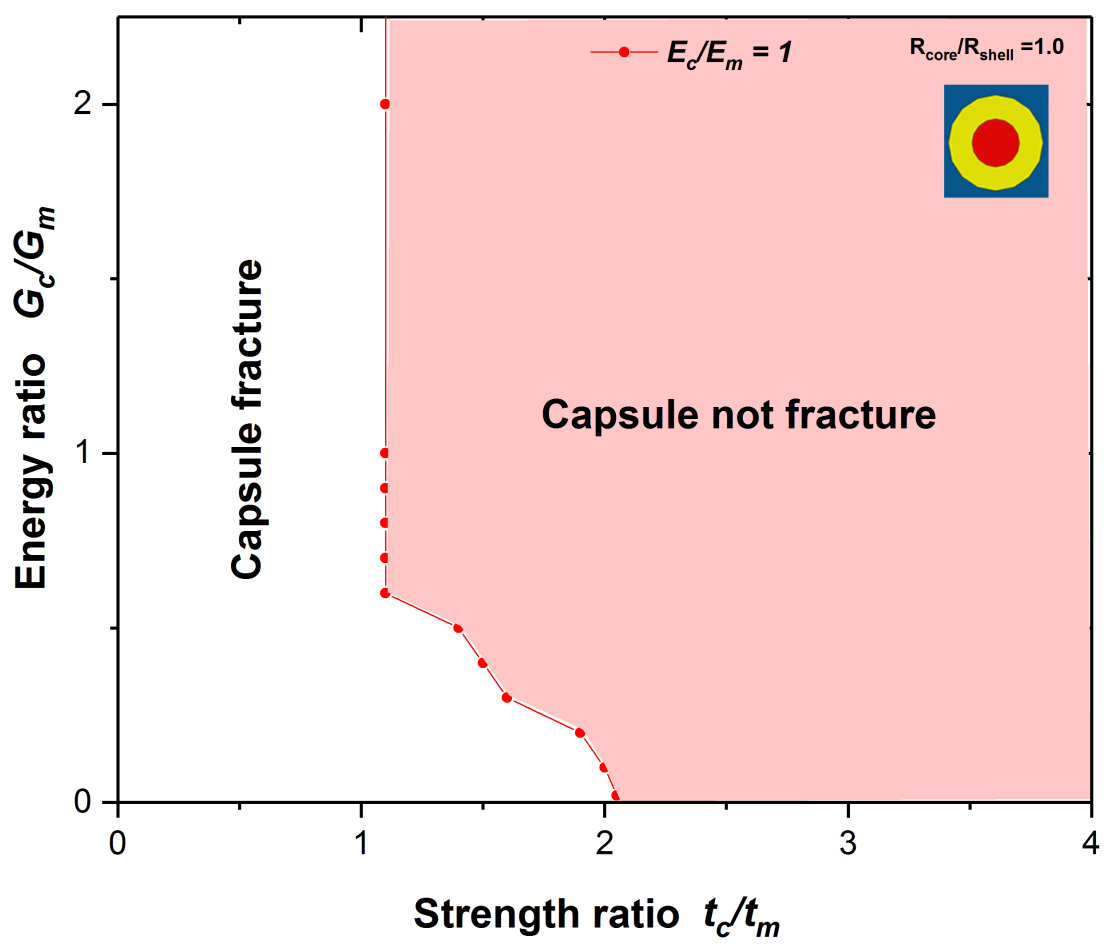

(a)

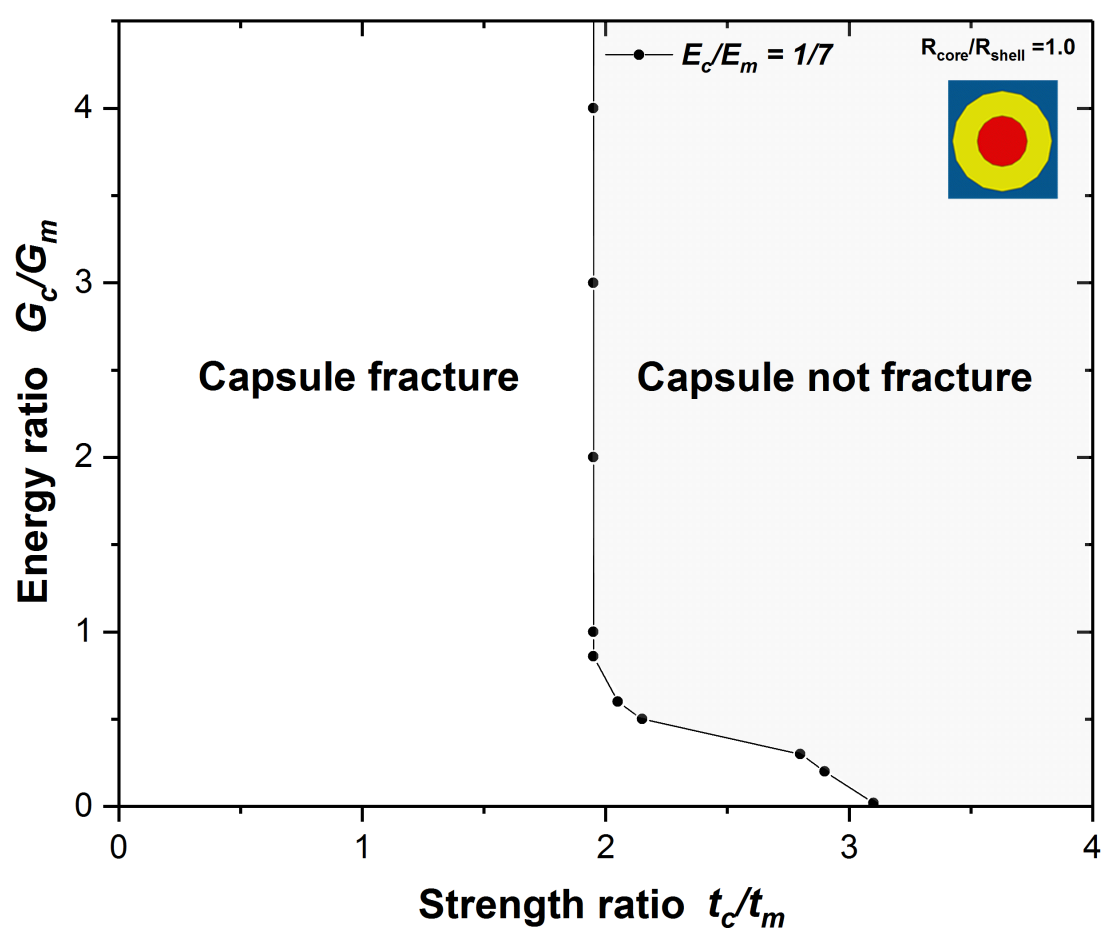

72 


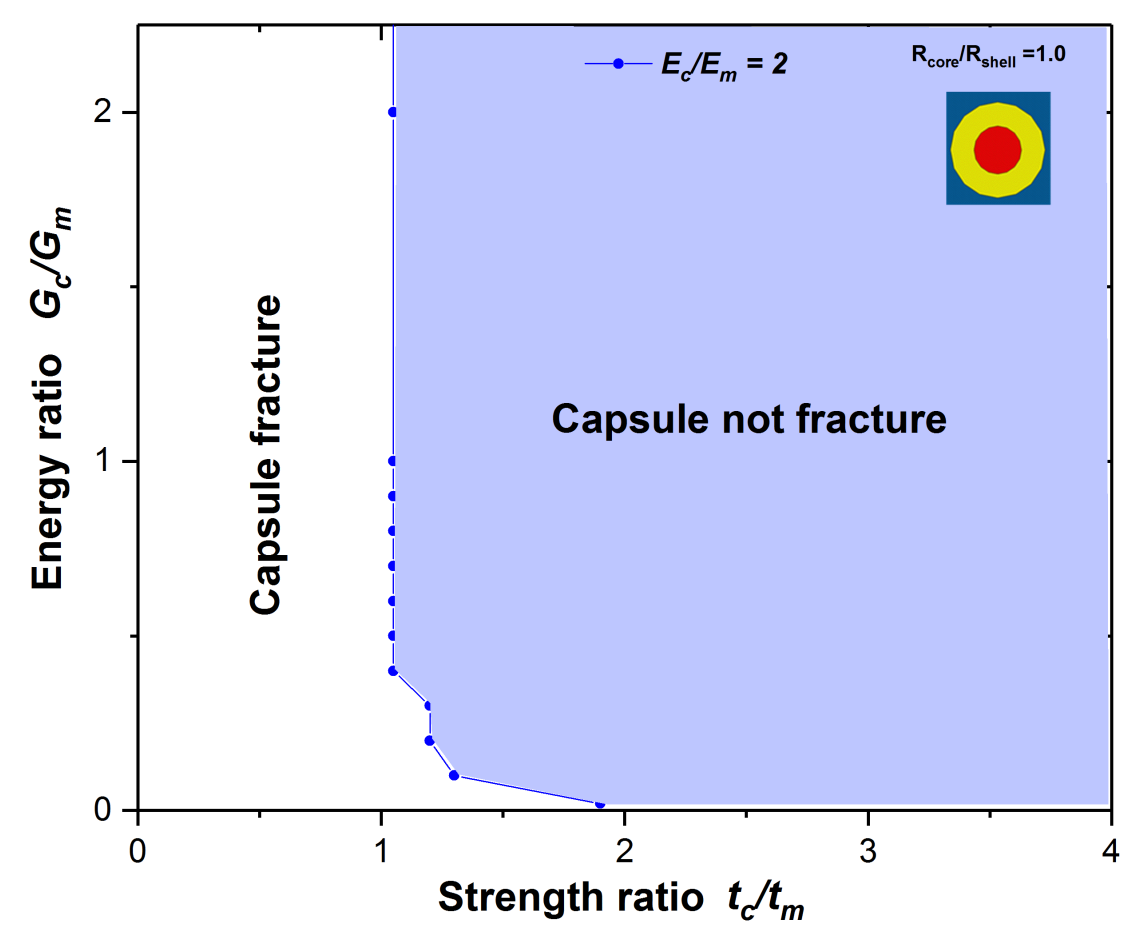

(c)

Figure 5.4: Effect of mismatch in elastic properties on fractured capsule. (a) $E_{c} / E_{m}=1$. (b) $E_{c} / E_{m}=1 / 7$. (c) $E_{c} / E_{m}=2$.

The comparison of corresponding regions between areas of capsule fracture and crack deflection from all elastic ratios can be seen in Fig. 5.5. As can be observed in Fig. 5.5, the regions of capsule fracture correspond to the left and bottom sides of the particular curve whereas the regions of crack deflection correspond to the right and top sides of the particular curve. A large number of simulations are extremely needed to define the curves shown in Fig. 5.5 with identifying not only the particular strength and energy ratios but also the transition phenomenon between two cases (capsule fracture and crack deflection) in each mismatch of elastic properties. 


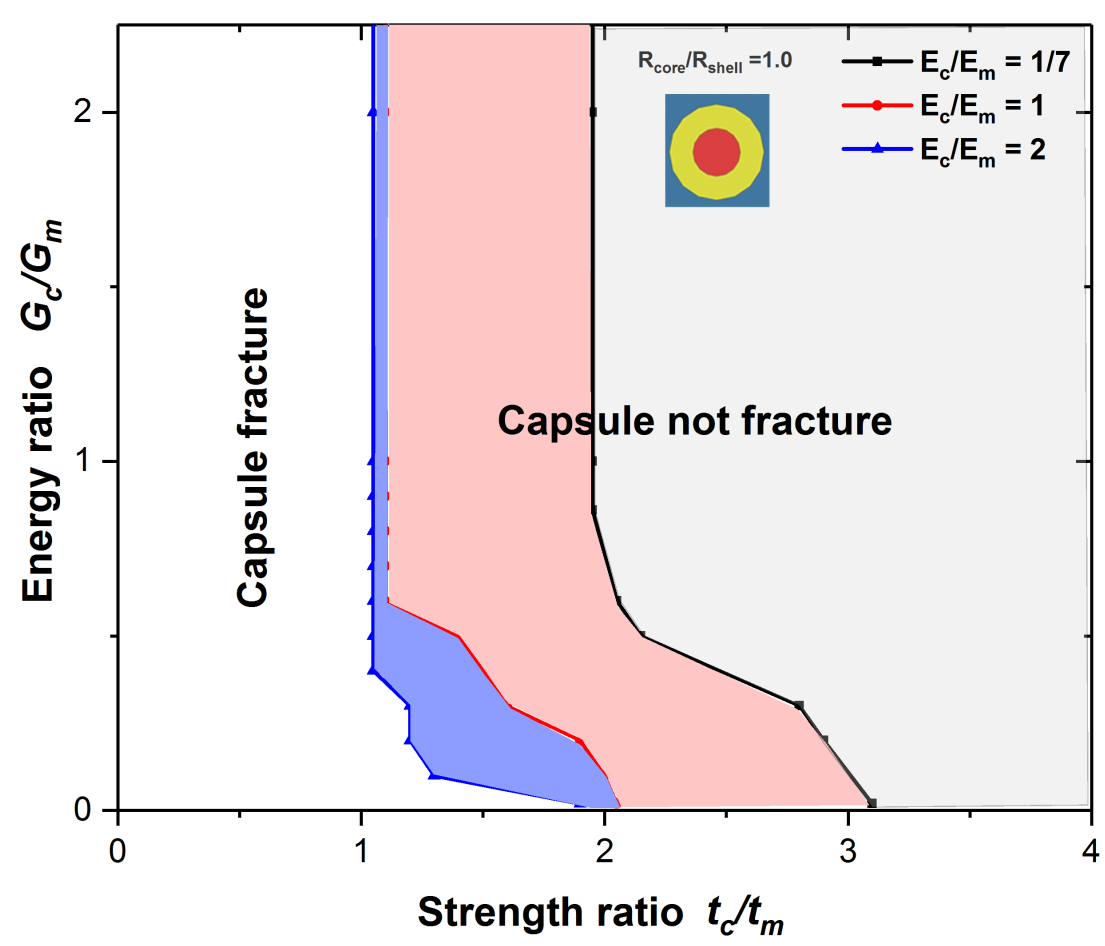

Figure 5.5: Fracture map: Effect of mismatch in fracture properties with different elastic ratios.

In general, the trend of capsule fracture is identified by a decrease of capsule stiffness whereas crack deflection is favored with an increase of capsule stiffness. This finding is in good agreement with the established conclusion found in the literature which indicates that an incoming crack will be attracted to a softer particle meanwhile a stiffer particle will cause a crack to be deflected far away from the particle. However, as shown in Fig. 5.5, the behavior of capsule fracture is strongly dependent on the mismatch in fracture properties, especially when using softer capsule (e.g., $E_{c} / E_{m}=1 / 7$ ) compared to the one with stiffer capsule (e.g., $E_{c} / E_{m}=2$ ). The interesting part that can be pointed out from fracture map shown in Fig. 5.5 is that capsule fracture occurs in softer capsule, even if the capsule strength is higher than the mortar matrix strength up to a factor of 2 or even more, given the energy ratio of capsule is adequately low. Regarding 
to stiffer capsule, capsule fracture is prevented if the capsule strength is higher than the mortar matrix strength by a factor of 1.1, regardless of lower fracture energy of the capsule.

\subsubsection{Effect of capsule wall thickness}

This section presents the simulation results to investigate the effect of capsule wall thickness on the fracture of capsule. An overview of parametric studies with highlighting different parameters will be explained at the first part along with the particular crack patterns. The fracture maps as results of the parametric studies will be created and summarized in the last section to differentiate the fracture mechanism of the capsule.

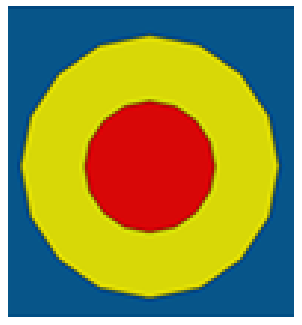

(a)

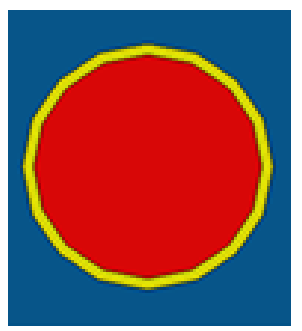

(c)

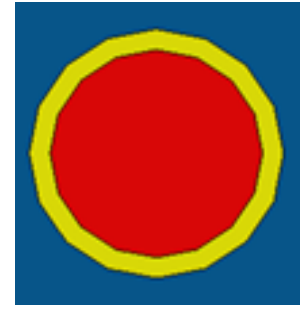

(b)

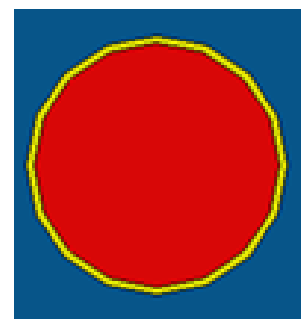

(d)

Figure 5.6: Capsule samples with different wall thickness. (a) R11. (b) R51. (c) R101. (d) R151. 


\section{The Effect of Mismatch Properties on Fractured Capsule}

\subsubsection{Fracture patterns}

All simulations presented here are based on the capsule core-wall thickness ratios previously explained as shown in Fig. 5.6. All crack patterns as illustrated in Fig. 5.7, which initiate from left to right, are distinguished from capsule thickness with different fracture properties ratios between the capsule and the mortar matrix. The elastic modulus ratios of $1 / 7$ between the capsule and the mortar matrix is used in all simulations regardless the capsule wall thickness. There are four different wall thickness cases considered in this study, namely Ratio 1:1 (Fig. 5.7a and b), Ratio 5:1 (Fig. 5.7c and d), Ratio 10:1 (Fig. 5.7e and f), and Ratio 15:1 (Fig. 5.7g and h). Capsule fractures in all thickness are observed when there is no mismatch in fracture properties between the capsule and the mortar matrix (Fig. $5.7 \mathrm{a}$ and g) and when the mismatch ratio in fracture properties up to 1.5 are used (Fig. 5.7c and e). When the fracture strength of the capsule is twice larger than the mortar matrix (Fig. $5.7 \mathrm{~d}$, f, and h), the incoming crack deflects the path along the capsule and could not penetrate into the shell whereas in other case, capsule fracture occurs when the small toughness ratio between the capsule and the mortar matrix is applied even though the largest thickness of capsule is used (Fig. 5.7b). Nonetheless, the mismatch in fracture energy (toughness) has significant effect on capsule fracture despite larger thickness of the capsule. 
5. The Effect of Mismatch Properties on Fractured Capsule

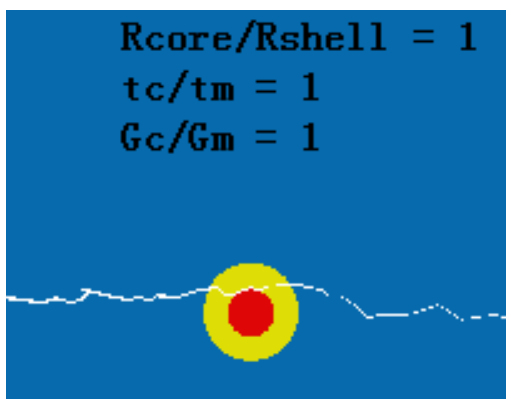

(a)

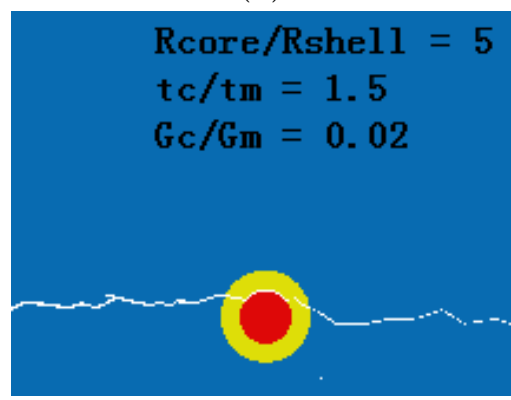

(c)

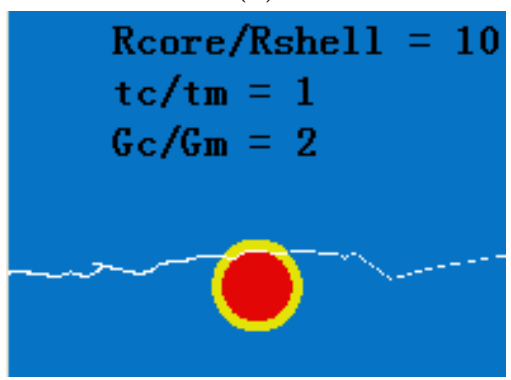

(e)

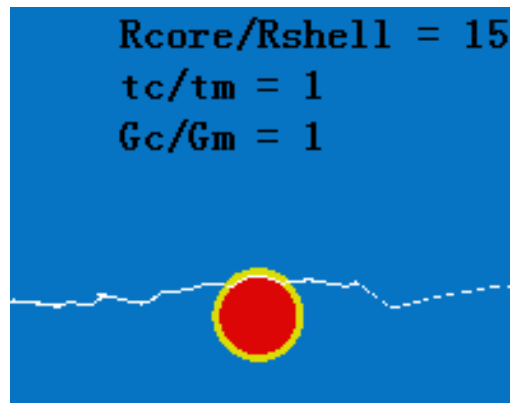

(g)

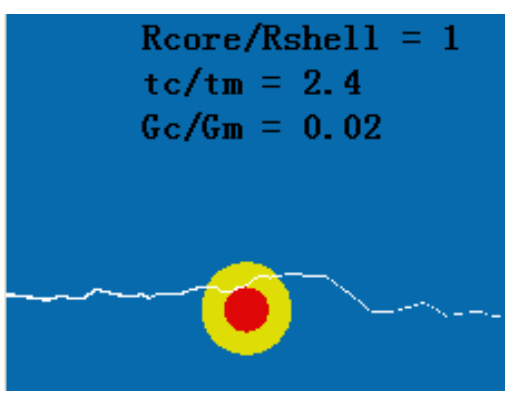

(b)

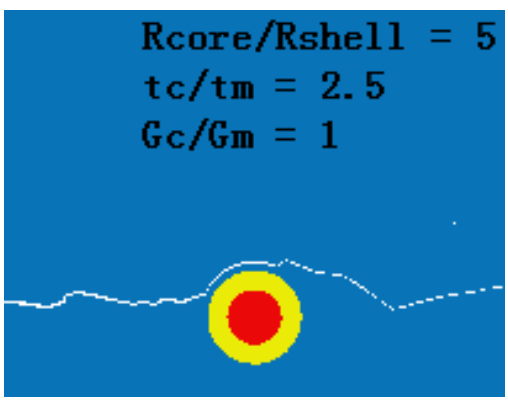

(d)

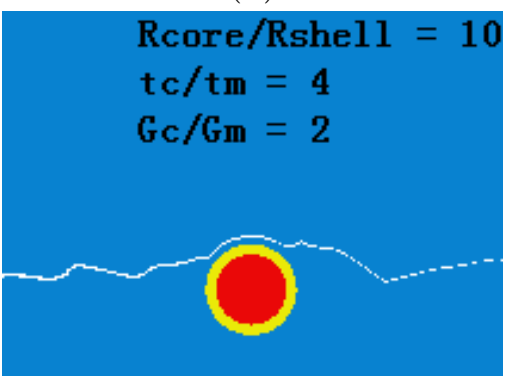

(f)

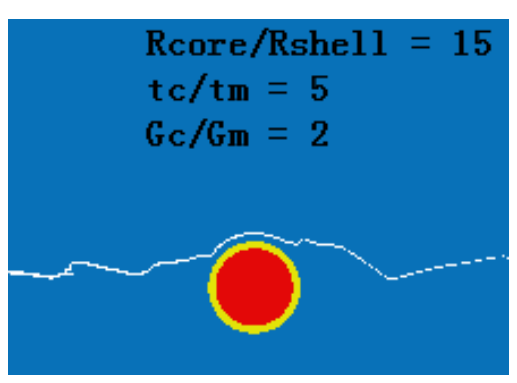

(h)

Figure 5.7: Failure path of an incoming crack through capsule with different core-wall thickness ratios. 


\section{The Effect of Mismatch Properties on Fractured Capsule}

\subsubsection{Fracture maps}

The effect of capsule wall thickness is investigated by considering four different wall thickness ratios, namely Ratio 1, 5, 10, and 15. In all presented simulations, the approaching crack has no initial offset from the center of the capsule (the crack lies directly in front of the center of the capsule). The results of these extensive simulations are summarized in the form of a fracture map which compare corresponding regions between areas of capsule fracture and crack deflection from all thickness ratios as shown in Fig. 5.8. As can be seen in Fig. 5.8, the regions of capsule fracture correspond to the left and bottom sides of the particular curve whereas the regions of crack deflection correspond to the right and top sides of the particular curve. A large number of simulations are significantly needed to determine the curves with identifying not only the particular strength and energy ratios but also the transition phenomenon between two cases (capsule fracture and crack deflection) in each thickness ratio. Consequently, it can be concluded from the fracture map (Fig. 5.8) that capsule shell thickness has a significant influence on the capsule fracture, but only in combination with the fracture properties (fracture strength and energy). 


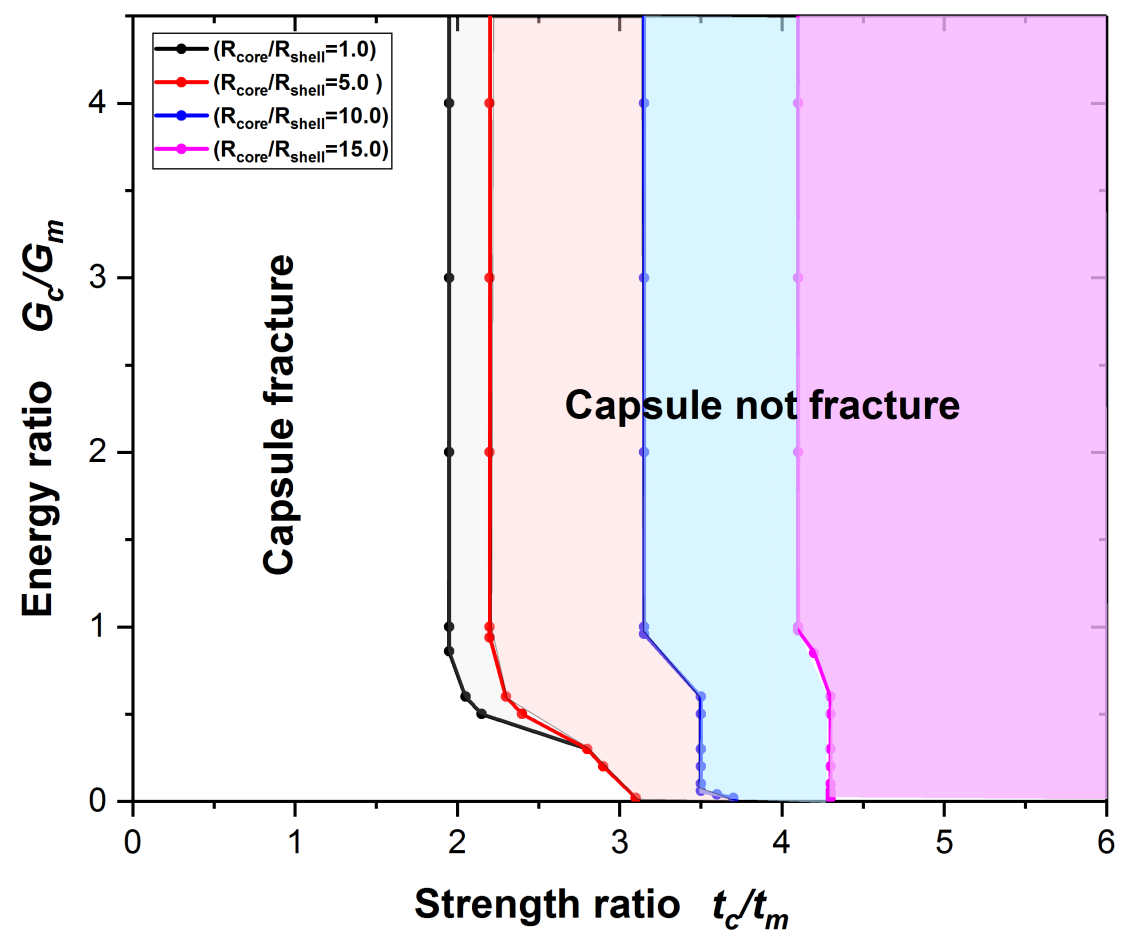

Figure 5.8: Fracture map: Effect of different capsule wall thickness ratios. 


\section{Chapter 6}

\section{D Numerical Simulations Under Uniaxial Tension}

The 3D numerical simulations of encapsulation-based self-healing concrete at mesoscale level is investigated in this section. At the mesoscale, self-healing concrete is treated as a three or four phases material consisting of aggregates, the mortar matrix, capsules and their interfaces. The mechanical behavior of self-healing concrete at the macroscale severely depends on its meso-structure. Hence, numerical modeling of self-healing concrete at the meso-scale becomes significant and was used in this study.

Some contributions studied the possibility of capsules hit by cracks both in 2D and 3D. Lv and Chen [2013] studied these possibilities for different volume fractions and capsule shell thickness [Gilabert et al., 2015]. A dosage analysis of self-healing agents required for capsules combined with probability theory was performed in Lv et al. [2014]. However, a full three-dimensional model might be necessary for reliable predictions, which will be the focus of this study. The 3D results will subsequently be compared to $2 \mathrm{D}$ results in order to highlight the necessity of 3D model. Most capsules are made of Poly Methyl Methacrylate-PMMA since they break easily even under small deformations and are strong enough to resist during mixing compared to other materials such as polystyrene (PS), poly lactic acid (PLA), and glass [Hilloulin et al., 2015]. The healing agent inside the capsule is modeled as solid element, hence the liquid flow into the crack area, its 


\section{3D Numerical Simulations Under Uniaxial Tension}

polymerization, and healing efficiency is not studied in this manuscript. To represent the complex fracture process in multi-phases medium, cohesive interface elements (CIEs) were pre-inserted into the discretization both at the interfaces and the mortar-matrix. The objectives of this study are: (i) to simulate the fracture process of capsule-based self-healing concrete in 3D and (ii) to quantify the influence of different volume fractions of capsules on the load-carrying capacity and fracture energy.

\subsection{Microstructures generation at mesoscale level}

\subsubsection{Aggregates and capsules generation}

The Fuller curve is often used to define the aggregate distribution in concrete [Wriggers and Moftah, 2006], which is categorized based on the sieve analysis into different numbers of segments. In this study, the aggregate size distributions from Hirsch [Hirsch, 1962] summarized in Table 6.1 is adopted. The cut-off size of $2.36 \mathrm{~mm}$ was taken as basis of the coarse aggregates and concrete was treated as multi-phase material consisting of mortar, aggregates, capsule walls and capsule cores. The coarse aggregates in normal concrete usually represent $30-50 \%$ of the total concrete volume.

Table 6.1: Aggregate size distributionHirsch [1962]

\begin{tabular}{llll}
\hline $\begin{array}{l}\text { Sieve } \\
(\mathrm{mm})\end{array}$ & size & $\begin{array}{l}\text { Total percent- } \\
\text { age retained } \\
(\%)\end{array}$ & $\begin{array}{l}\text { Total percent- } \\
\text { age passing } \\
(\%)\end{array}$ \\
\hline 12.70 & 0 & 100 & \\
9.50 & 39 & 61 \\
4.75 & 90 & 10 \\
2.36 & 98.6 & 1.4 \\
\hline
\end{tabular}

Based on X-ray tomographic images, it was found that the coarse aggregates have circular and elliptical shapes in 2D or spherical and ellipsoidal shapes in 3D [Daudeville, 2014]. Du et al. [2014] reported that the shape of the aggregates has no significantly influence on the macroscopic behavior of concrete. For the sake of 


\section{3D Numerical Simulations Under Uniaxial Tension}

simplicity, we therefore assume spherical shapes for the aggregates. We also generate 2D models for comparison assuming circular aggregates. Experiments show that the agitation rates during the process determine the size and the thickness of the capsules. The diameter range of capsules commonly range from $1 \mathrm{~mm}$ to 8 mm [Gruyaert et al., 2016; Hilloulin et al., 2015] and the results presented in this study based on a diameter of $2.0 \mathrm{~mm}$. In addition, the core-wall thickness ratio of 1 was applied considering the capsules should have strong resistance during the mixing process [Hilloulin et al., 2015].

\subsubsection{Packing algorithms}

The identical packing algorithm to generate the meso-structure in concrete as explained in the previous section is adopted. The basic idea of the procedure is to create randomly aggregates and capsules repeatedly until the target area or volume is achieved.

A series of meso-scale models with dimensions of $25 \mathrm{~mm} \times 25 \mathrm{~mm}$ in $2 \mathrm{D}$ and 25 $\mathrm{mm} \times 25 \mathrm{~mm}$ x $25 \mathrm{~mm}$ in 3D are generated, see Figs. 6.1 and 6.2, respectively. An aggregate volume fraction of $30 \%$, see Table 6.1, is assumed constant along with the variation capsule volume of fraction. The minimum space allowed between the boundary of the concrete model and the edge of the aggregates or capsules is $0.5 \mathrm{~mm}$. 


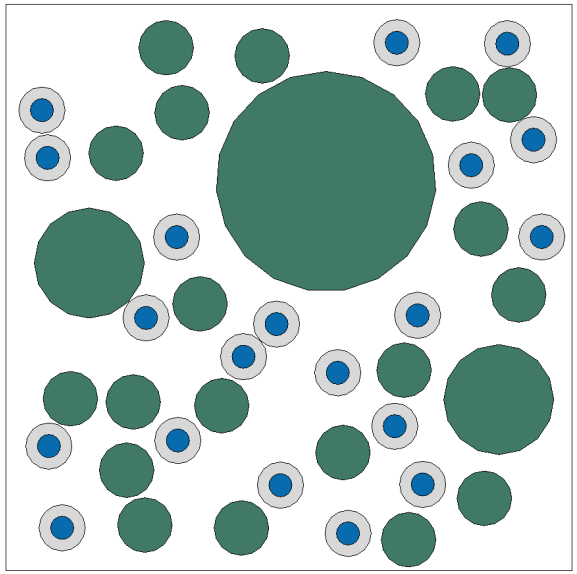

(a)

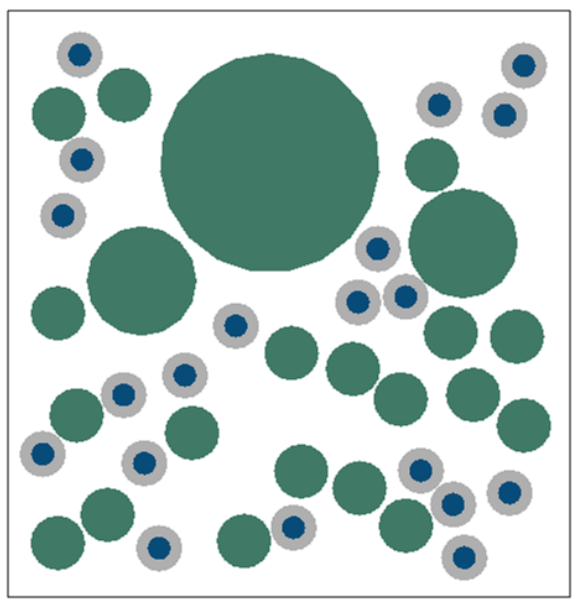

(c)

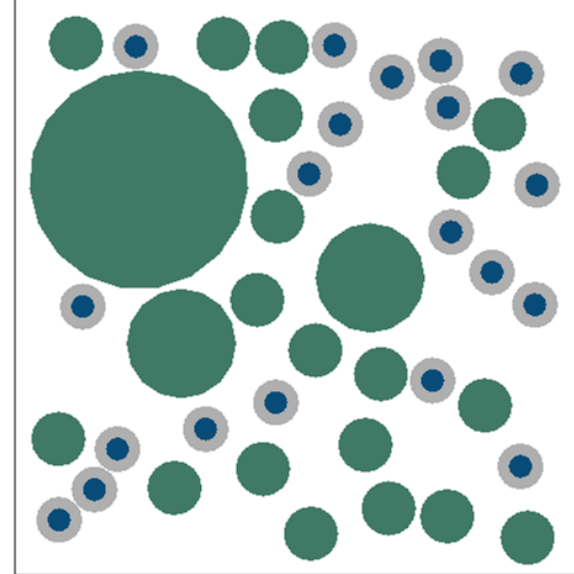

(b)

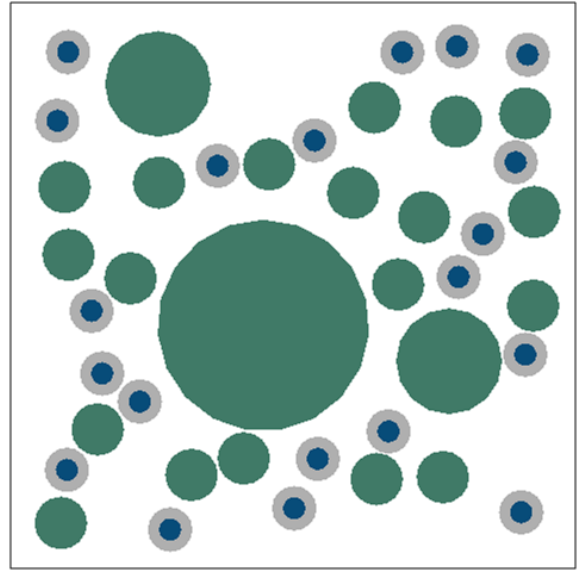

(d)

Figure 6.1: Numerically generated 2D mesomodels with different realizations for capsule-based self-healing concrete (volume fraction of capsules $=2 \%$ ) 


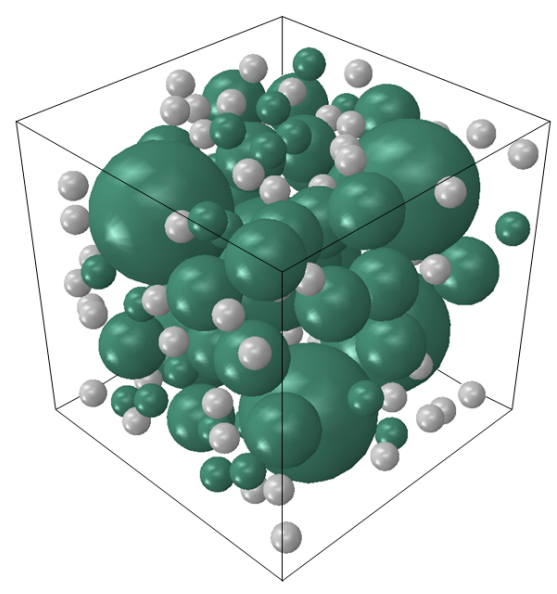

(a)

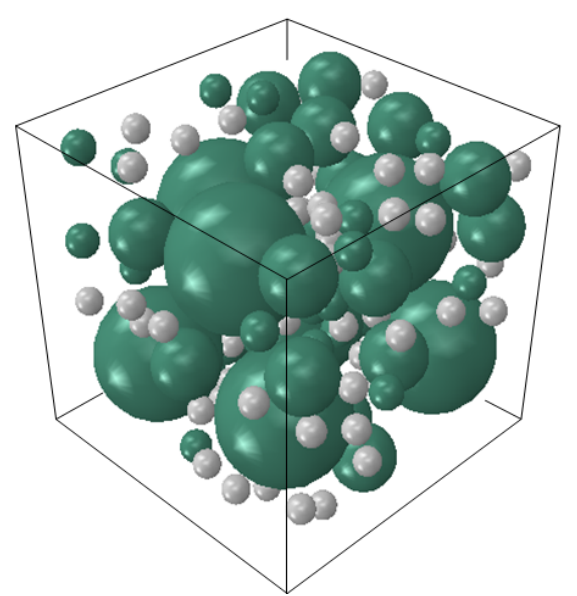

(c)

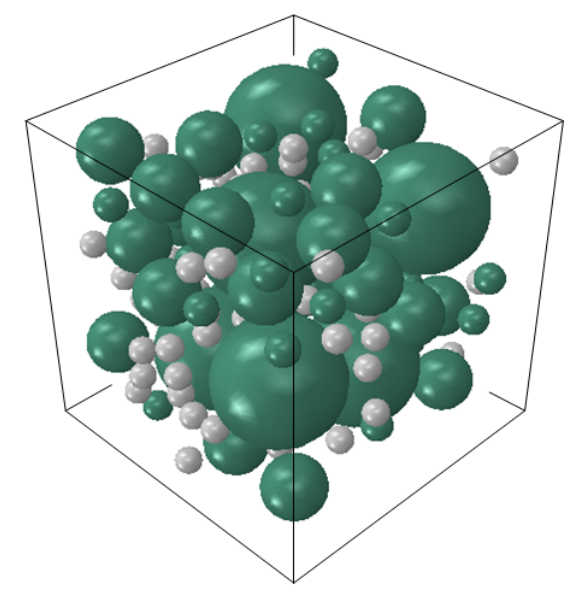

(b)

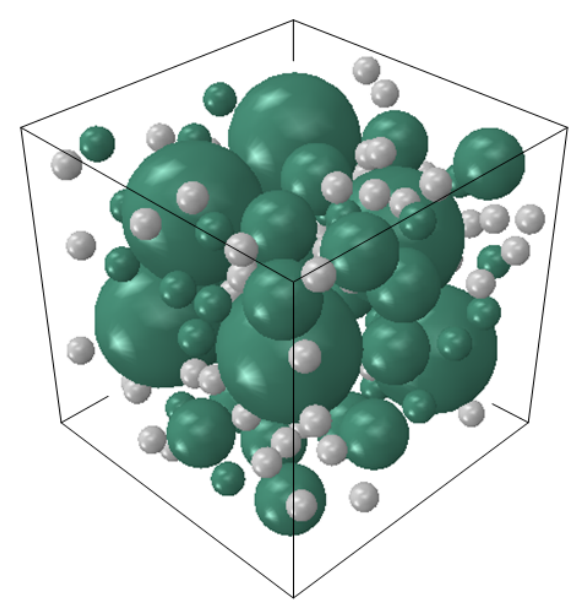

(d)

Figure 6.2: Numerically generated 3D mesomodels with different realizations for capsule-based self-healing concrete (volume fraction of capsules $=2 \%$ )

\subsection{Cohesive zone model}

A bilinear cohesive zone model (CZM) as illustrated in Fig. 6.3 is used. The cohesive elements are based on the cohesive crack model available in ABAQUS, and its constitutive behavior can be described by tension/shear softening laws. This model is readily available in ABAQUS which is used for all computational studies. 


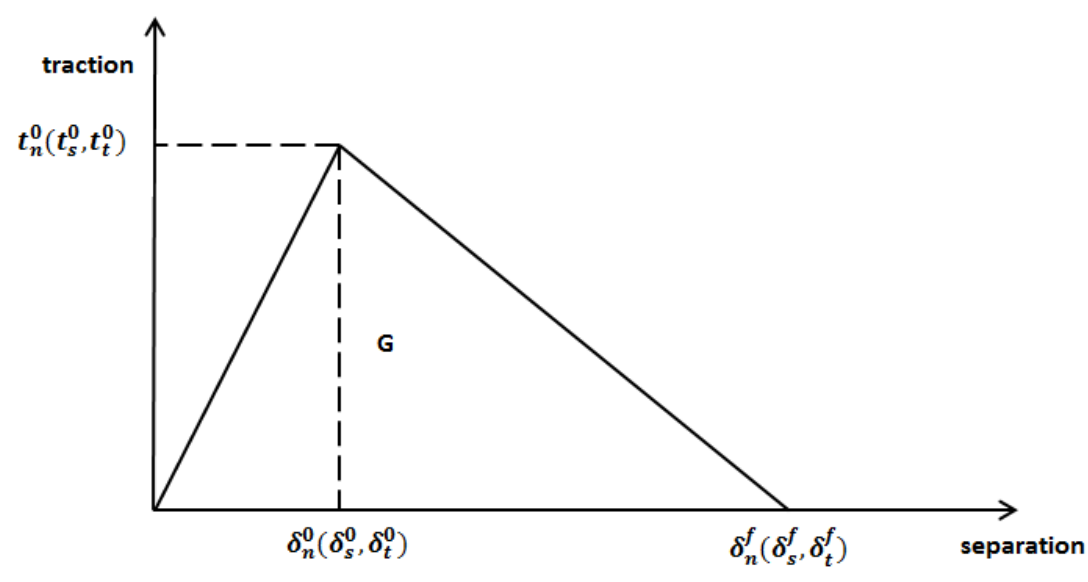

Figure 6.3: bilinear traction-separation law for cohesive elements, reproduced from Simulia [2013]

The cohesive zone model in ABAQUS requires two input parameters, i.e. the fracture energy

$$
G=\int_{0}^{\delta_{f}} t(\delta) d \delta=\frac{1}{2} t_{o} \delta_{f}
$$

and the cohesive strength $t_{o} ; \delta_{f}$ is the separation displacement. The effective relative displacements $\delta_{m}$ can be determined after considering the effects of displacements in normal $\left(\delta_{n}\right)$ and two shear directions $\left(\delta_{s}\right.$ and $\left.\delta_{t}\right)$ :

$$
\delta_{m}=\sqrt{\left\langle\delta_{n}\right\rangle^{2}+\delta_{s}^{2}+\delta_{t}^{2}}
$$

where \langle\rangle is the Macaulay bracket and

$$
\left\langle\delta_{n}\right\rangle=\left\{\begin{array}{lll}
\delta_{n}, & \delta_{n} \geq 0 & (\text { tension }) \\
0, & \delta_{n}<0 \quad & (\text { compression })
\end{array}\right.
$$

A damage variable $D$ is introduced to describe the bilinear traction-separation low, where the damage evolution is expressed as

$$
D=\frac{\delta_{m f}\left(\delta_{m, \max }-\delta_{m 0}\right)}{\delta_{m, \max }\left(\delta_{m f}-\delta_{m 0}\right)}
$$

$\delta_{m f}$ indicating the effective relative displacements at failure, $\delta_{m, \max }$ denotes the 


\section{3D Numerical Simulations Under Uniaxial Tension}

maximum relative displacement obtained during the loading history, and $\delta_{m, 0}$ is the effective relative displacement at initial damage.

It is obvious from Eq. 6.4 that the damage will evolve gradually from 0 to 1 during loading. In this study, damage in the cohesive element was assumed to be initiated when a quadratic function including the ratio of nominal stress reaches a value of one as given by

$$
\left\{\frac{\left\langle t_{n}\right\rangle}{t_{n 0}}\right\}^{2}+\left\{\frac{t_{s}}{t_{s 0}}\right\}^{2}+\left\{\frac{t_{t}}{t_{t 0}}\right\}^{2}=1
$$

where $t_{n}, t_{s}$, and $t_{t}$ are the traction in normal, shear, and tangential directions, respectively. More details about the cohesive traction model can be found in the ABAQUS manual.

The concrete samples were meshed with 3-node triangular solid elements (CPS3) for our 2D models (used for comparison to 3D results) and 4-node tetrahedra solid elements (C3D4) in 3D. To represent cracks, 4-node (COH2D4) and 6-node (COH3D6) cohesive elements were pre-inserted into the initial generated element interfaces in 2D and 3D, respectively. Six sets of cohesive interface elements (CIEs) are automatically generated, namely, inside the aggregates (CIE-AA), inside the mortar matrix (CIE-MM), inside the capsule shells (CIE-SS), inside the capsule cores (CIE-CC), the aggregate and the mortar matrix interfaces (CIE$\mathrm{AM}$ ), the capsule cores and the shells interfaces (CIE-CS), and the capsule shells and the mortar interfaces (CIE-SM).

\subsection{Model parameters}

The 2D concrete specimens assuming plane strain conditions as well as 3D specimens under uniaxial tension as illustrated in Fig.6.4 are considered. Their dimensions are $25 \mathrm{~mm} \times 25 \mathrm{~mm}$ in $2 \mathrm{D}$ and $25 \mathrm{~mm} \times 25 \mathrm{~mm} \times 25 \mathrm{~mm}$ in 3D. A uniformly distributed horizontal displacement is applied on the right surface of the specimen. Vertical displacements of all nodes on the left surface are free, except the ones at the lower corner of the specimen, which are fixed to prevent rigid body motion. All material properties used in our simulations can be found in Table 6.2; they are adopted from Gilabert et al. [2015]; Hilloulin et al. [2015]; 


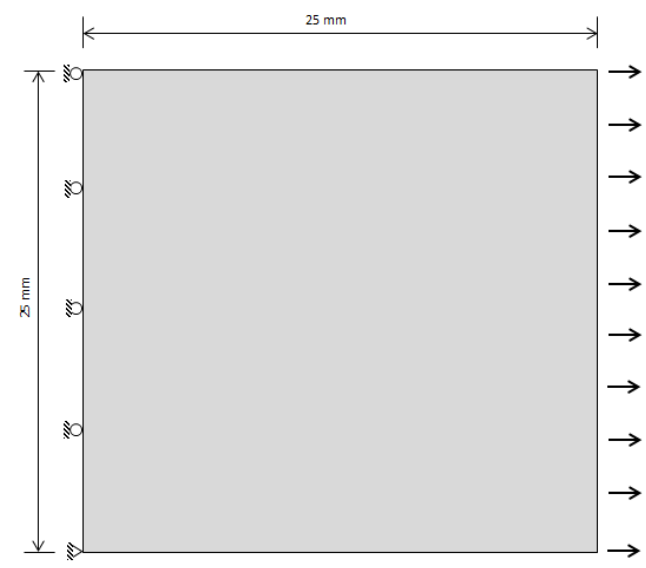

(a) Schematic 2D model

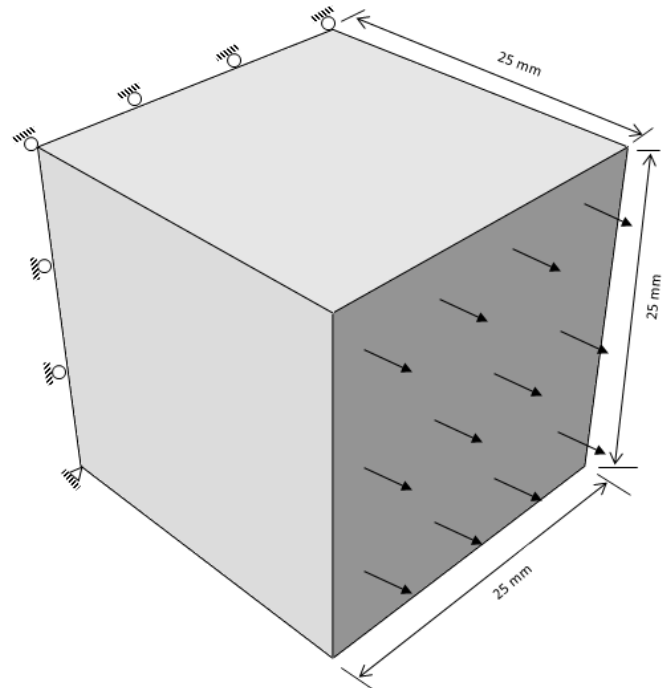

(b) Schematic 3D model

Figure 6.4: Geometry dimensions, boundary, and loading conditions

Keller and Sottos [2006]; Matbase; Wang et al. [2013]; Wang and Jivkov [2015].

Table 6.2: Material properties

\begin{tabular}{lcccccc}
\hline Model & $\begin{array}{c}\text { Young's } \\
\text { modulus E } \\
(\mathrm{MPa})\end{array}$ & $\begin{array}{c}\text { Poisson's } \\
\text { ratio }\end{array}$ & $\begin{array}{c}\text { Density } \\
\left(10^{-9}\right. \\
\left.\text { tonne } / \mathrm{mm}^{3}\right)\end{array}$ & $\begin{array}{c}\text { Elastic } \\
\text { stiffness } \\
k_{n}(\mathrm{MPa} / \mathrm{mm})\end{array}$ & $\begin{array}{c}\text { Cohesive } \\
\text { strength } \\
t_{n}(\mathrm{MPa})\end{array}$ & $\begin{array}{c}\text { Fracture } \\
\text { energy }\end{array}$ \\
\hline Aggregate & 70000 & 0.2 & 2.5 & - & - & - \\
Mortar & 25000 & 0.2 & 2.2 & - & - & - \\
Capsule core & 1000 & 0.45 & 1.0 & - & - & - \\
Capsule shell & 3600 & 0.3 & 1.0 & - & - & - \\
CIE-AA & - & - & 2.5 & $10^{6}$ & - & - \\
CIE-AM & - & - & 2.2 & $10^{6}$ & 3 & 0.03 \\
CIE-MM & - & - & 2.2 & $10^{6}$ & 6 & 0.06 \\
CIE-CC & - & - & 1.0 & $10^{6}$ & - & - \\
CIE-CS & - & - & 1.0 & $10^{6}$ & 10 & 0.1 \\
CIE-SS & - & - & 1.0 & $10^{6}$ & 10 & 0.1 \\
CIE-SM & - & - & 1.0 & $10^{6}$ & 10 & 0.1 \\
\hline
\end{tabular}




\section{3D Numerical Simulations Under Uniaxial Tension}

Aggregates will have a higher strength than the mortar matrix and their interfaces. No cracks inside the aggregates are assumed. The healing liquid inside the capsule is modelled as linear elastic solid. The cracks are only allowed to initiate through capsule shell, not inside capsule core. The capsule shell properties made of urea formaldehyde are adopted from experimental data [Keller and Sottos, 2006; Wang et al., 2013], whereas the fracture properties are taken from recent experimental studies [Gilabert et al., 2015; Hilloulin et al., 2015; Wang and Jivkov, 2015]. For simplicity and due to lack of information, the shear behavior of fracture is assumed to be equal to the normal one.

\subsection{Numerical results}

\subsubsection{Mesh dependency study}

The first step in numerical simulations is determining the influence of the discretization on the results - for three different meshes, namely, coarse mesh, medium mesh, and fine mesh, with element length Le $=2.0 \mathrm{~mm}, 1.5 \mathrm{~mm}$, and $1.0 \mathrm{~mm}$, respectively as illustrated in Fig.6.5. The coarse mesh has 318,704 solid elements and 636,263 CIEs, the medium mesh 354,651 solid elements and 687,313 CIEs, and the fine mesh 422,854 solid elements and 841,401 CIEs, respectively. 


\section{3D Numerical Simulations Under Uniaxial Tension}

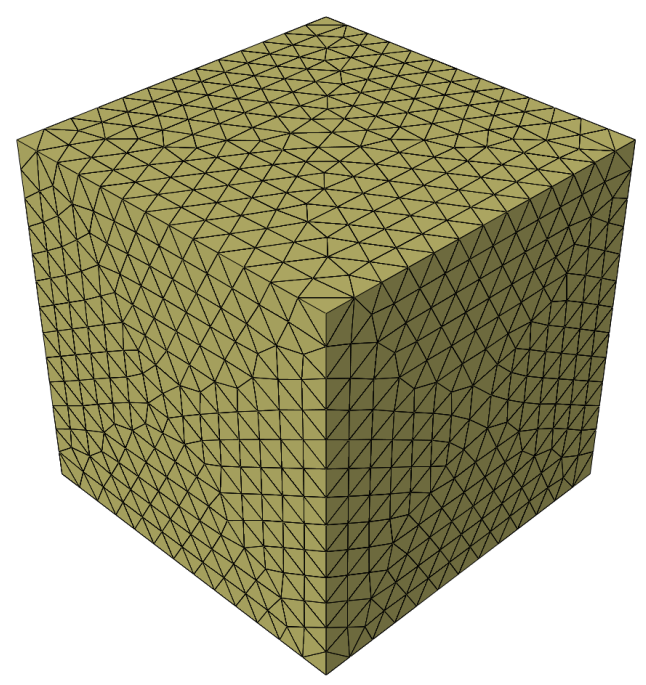

(a) Coarse mesh

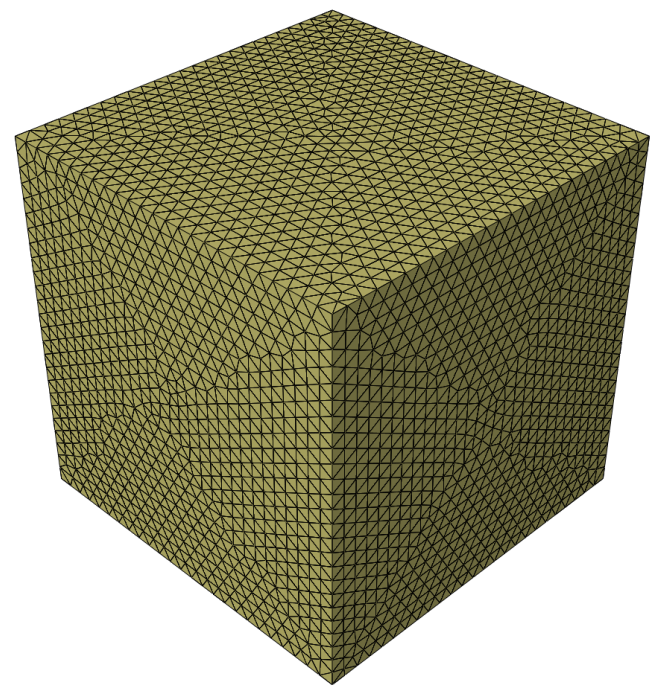

(c) Fine mesh

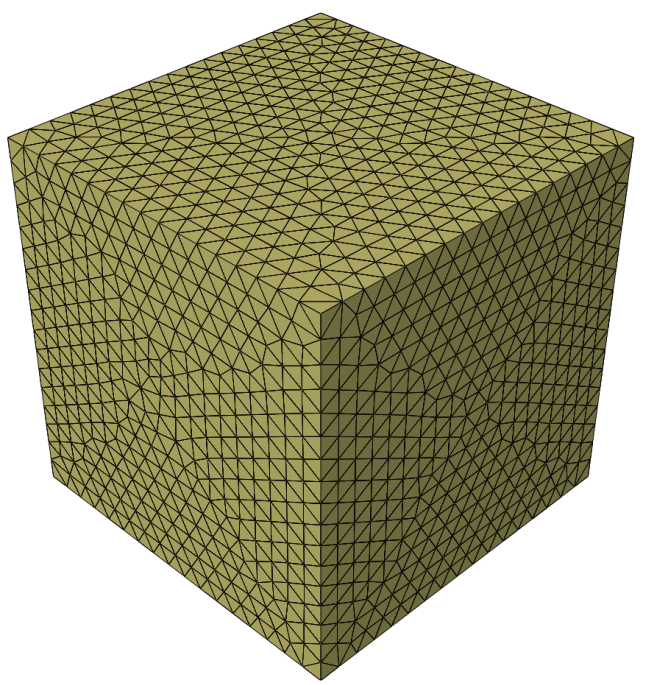

(b) Medium mesh

Figure 6.5: Different meshes densities used for mesh-dependence study

Fig. 6.6 and Fig. 6.7 show very similar results for the medium and the fine mesh. The stress value was obtained by dividing the total horizontal reaction forces with the cross sectional area of the specimen. 


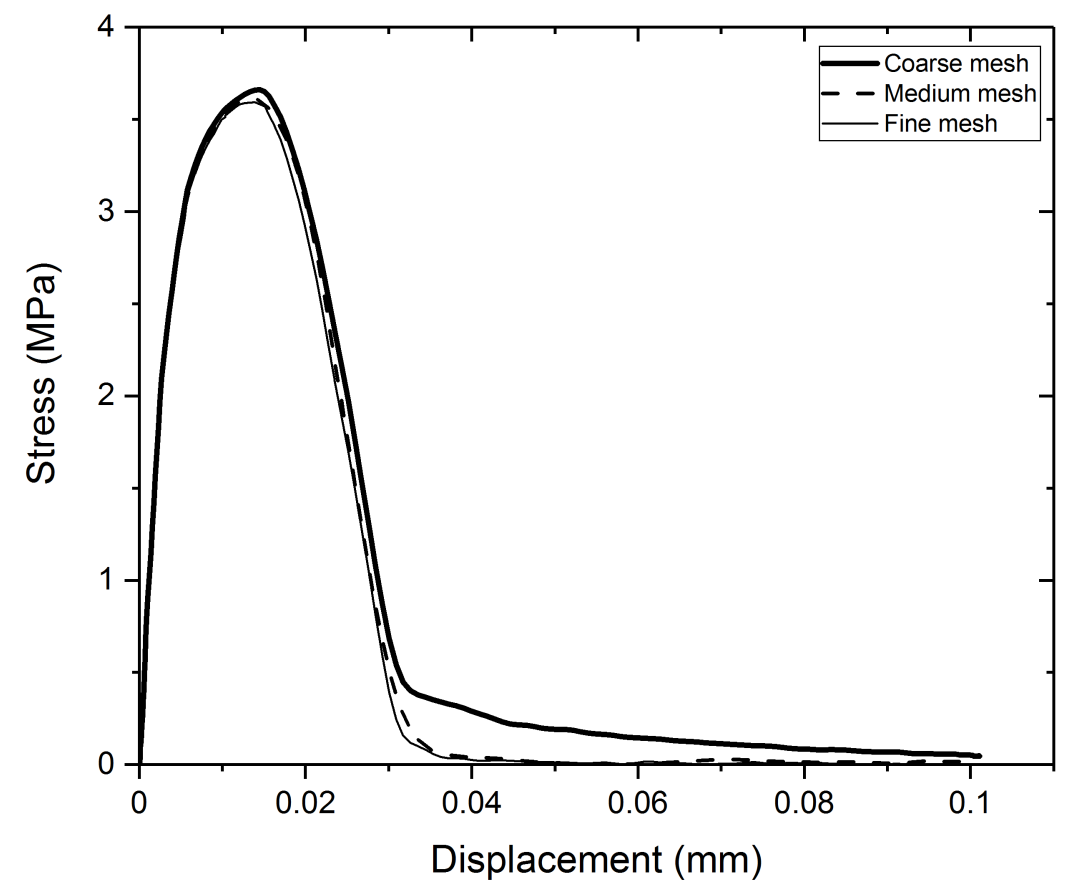

Figure 6.6: Stress-displacement curves from different meshes densities (volume fraction of capsules $=2 \%$ ) 


\section{3D Numerical Simulations Under Uniaxial Tension}

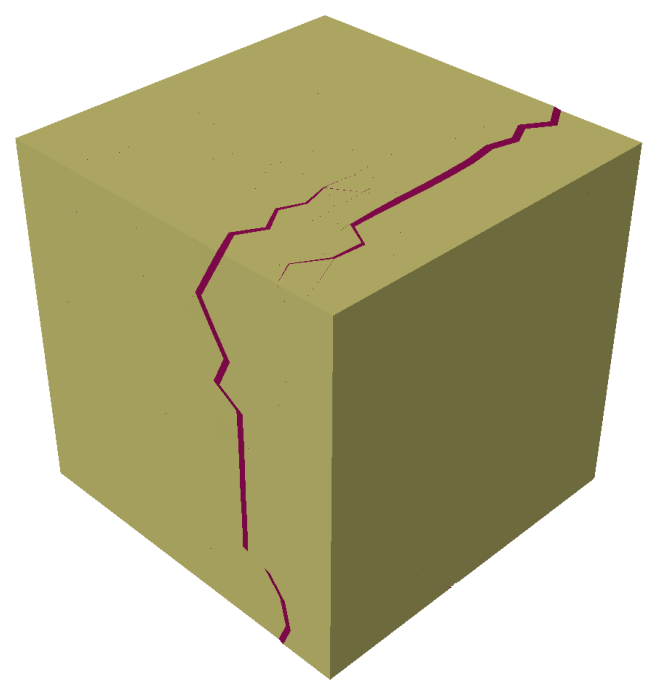

(a) Coarse mesh

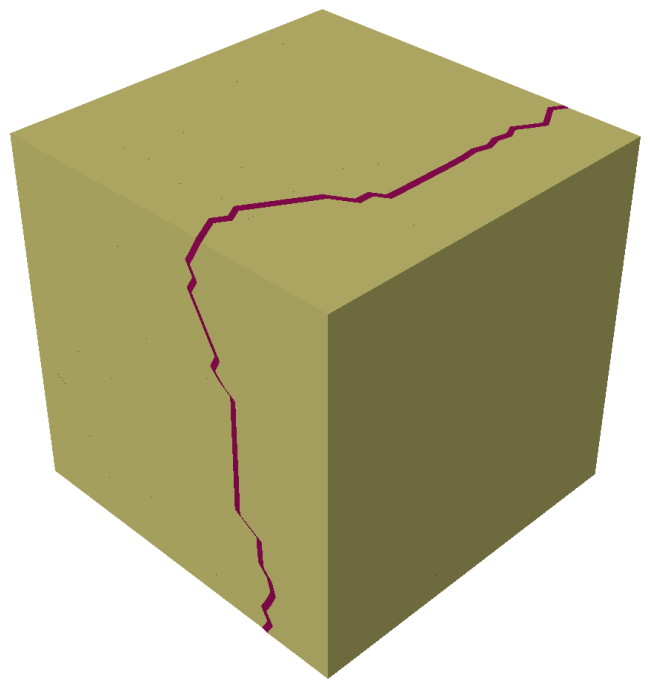

(c) Fine mesh

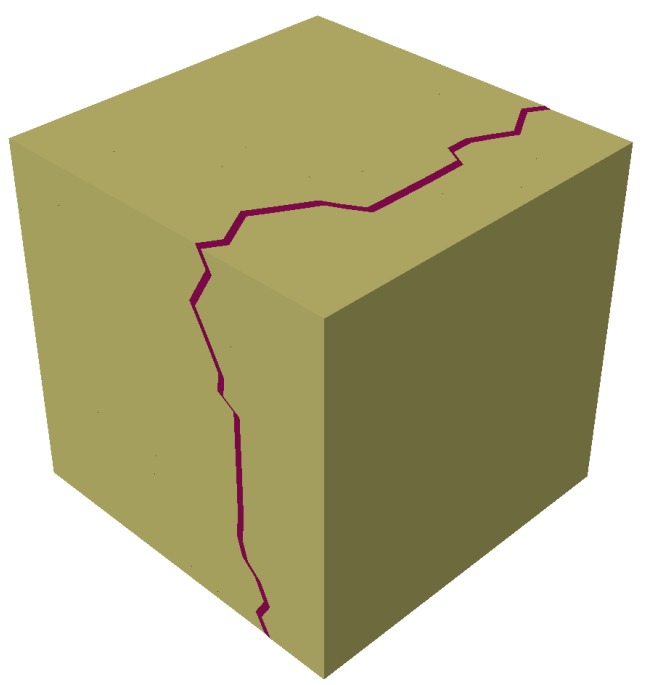

(b) Medium mesh

Figure 6.7: Final crack surfaces from different meshes densities

The medium and fine meshes exhibit also similar fracture patterns where only one single macrocrack occurs at the final stage of fracture. On the other hand, the coarse mesh shows two macro-cracks (Fig. 6.7b and c). For the subsequent analysis, discretizations with an element length of Le $=1.25 \mathrm{~mm}$ are employed. Fig. 6.8 depicts exemplary stress-displacement curves for 4 different realizations. Similar results were obtained for other realizations. 


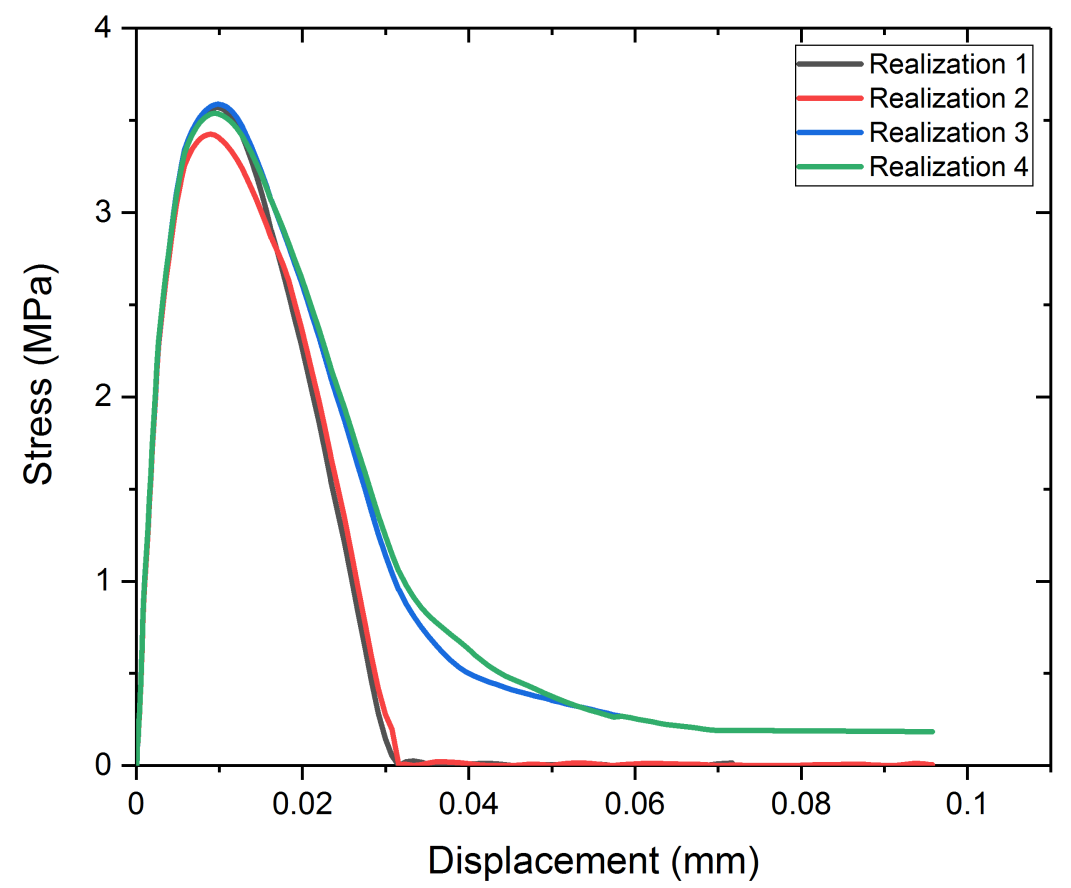

Figure 6.8: Stress-displacement curves from different realization microstructures with the same mesh densities (volume fraction of capsules $=2 \%$ )

\subsubsection{Tensile behavior}

Fig. 6.9 and Fig. 6.10 show the curves of the mean stress and mean dissipation energy for both 2D and 3D numerical simulation with a capsule volume fraction of $5 \%$, respectively. Both curves in Fig. 6.9 are qualitatively identical but show significant quantitative differences, which is probably due to the different dimensions (2D versus 3D). Or more precise: We believe the increase in the ultimate stress and fracture dissipation energy or decrease in standard deviation are due to thickness effects and less smooth fracture surfaces in the 3D model (see section 4.5 Crack patterns). Furthermore, in the softening region (see point $\mathrm{Y}$ at Fig. $6.11)$, the standard deviation of the $3 \mathrm{D}$ samples $(0.52 \mathrm{MPa})$ is larger compared to the $2 \mathrm{D}$ samples $(0.42 \mathrm{MPa})$. 


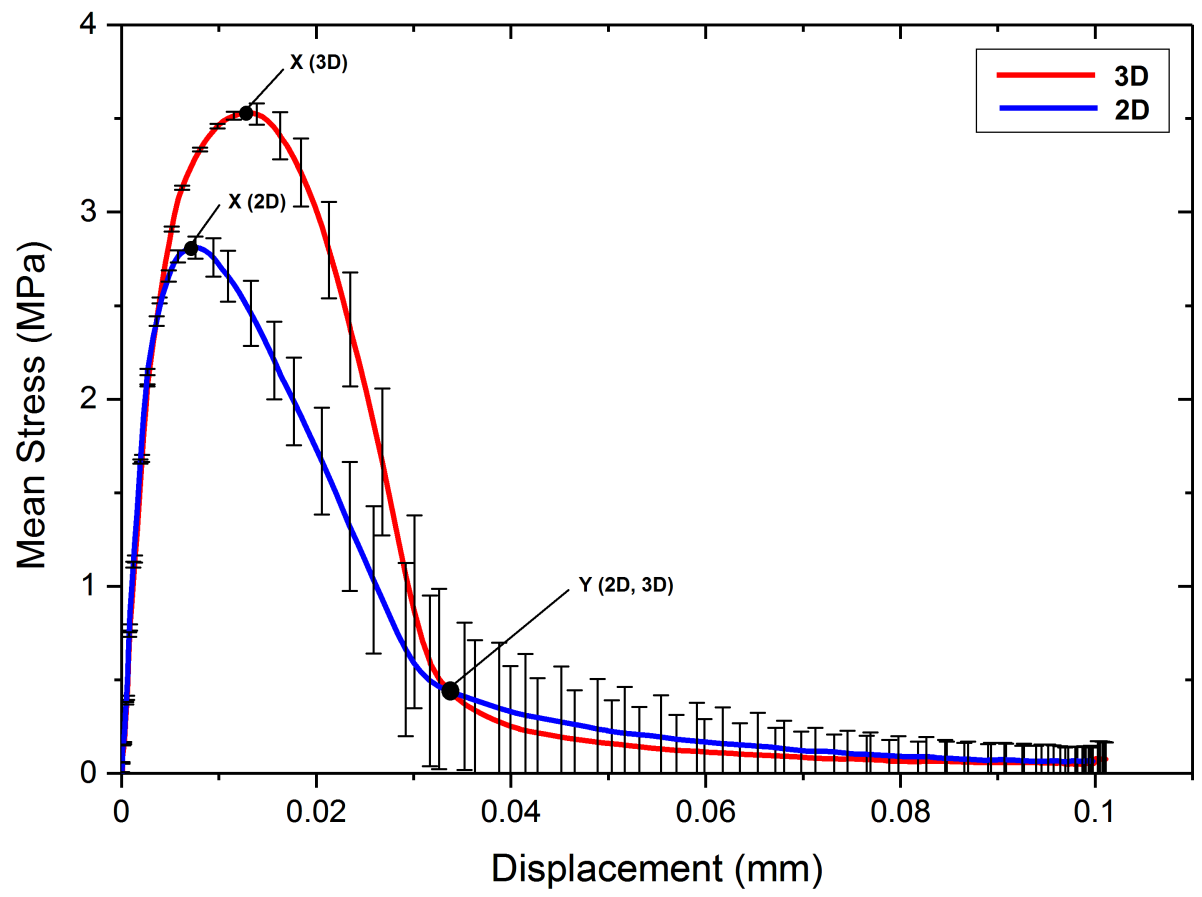

Figure 6.9: Mean stress-displacement curves from statistical analysis of 50 random samples (volume fraction of capsules $=5 \%$ ) 


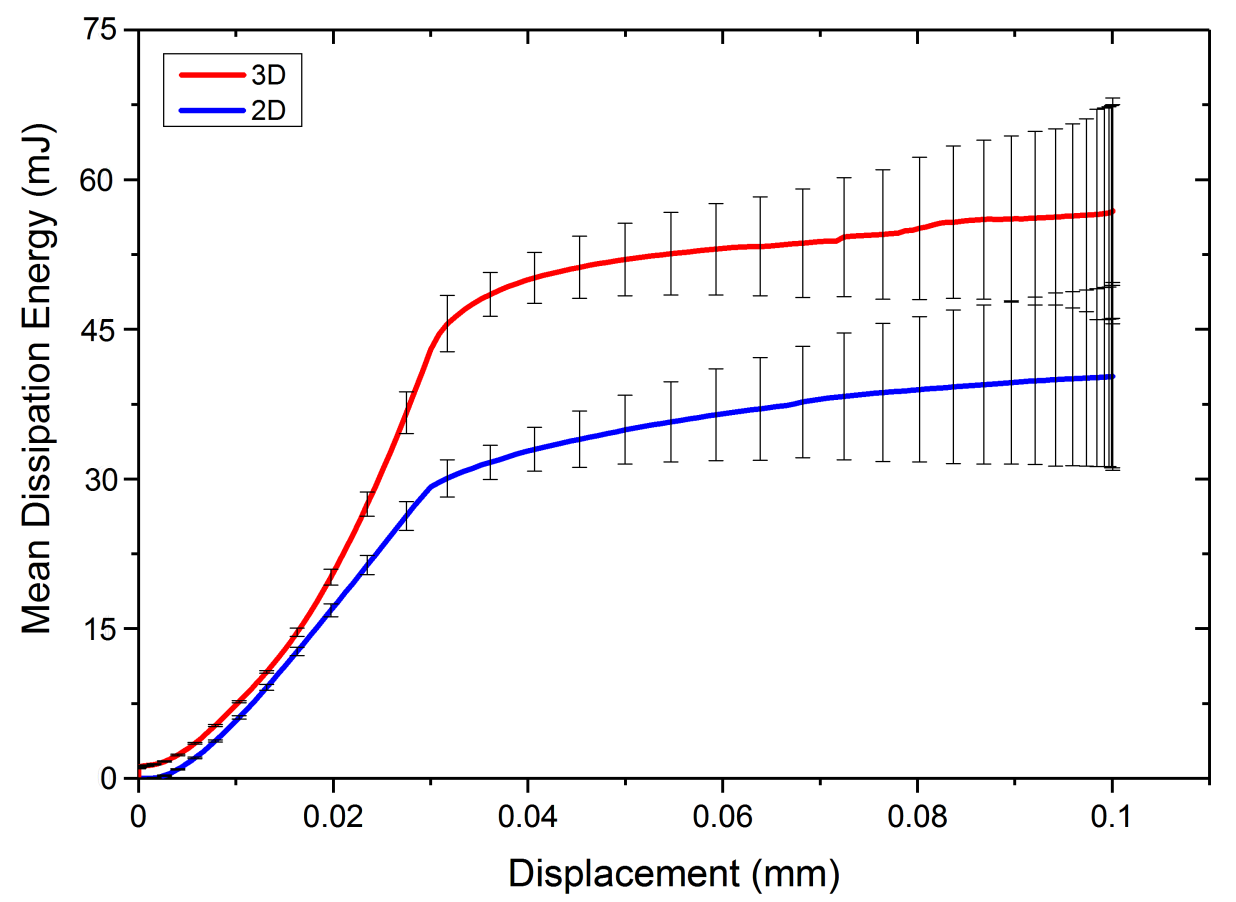

Figure 6.10: Mean dissipation energy curves from statistical analysis of 50 random samples (volume fraction of capsules $=5 \%$ )

The mean ultimate stress predicted by the $2 \mathrm{D}$ and $3 \mathrm{D}$ models are $2.75 \mathrm{MPa}$ and 3.50 MPa, respectively, which is a $27,3 \%$ increase. Similarly, the mean dissipation energy predicted by $2 \mathrm{D}$ and $3 \mathrm{D}$ models are $41.5 \mathrm{~mJ}$ and $57.5 \mathrm{~mJ}$, respectively, which is a difference of $38.6 \%$ (Fig. 6.10). This higher value is attributed to the different post-localization behavior. Fig. 6.11 shows the standard variation in the stress versus the displacement for both 2D and 3D samples. The standard deviation at the peak stress from 2D modeling is $0.095 \mathrm{MPa}$, whereas for $3 \mathrm{D}$ modeling is $0.045 \mathrm{MPa}$ (see point $\mathrm{X}$ at Fig. 6.11). The results suggest the use of a 3D model for the capsules-based self-healing concrete. Therefore, the subsequent results presented in this study are based only on 3D simulations. 


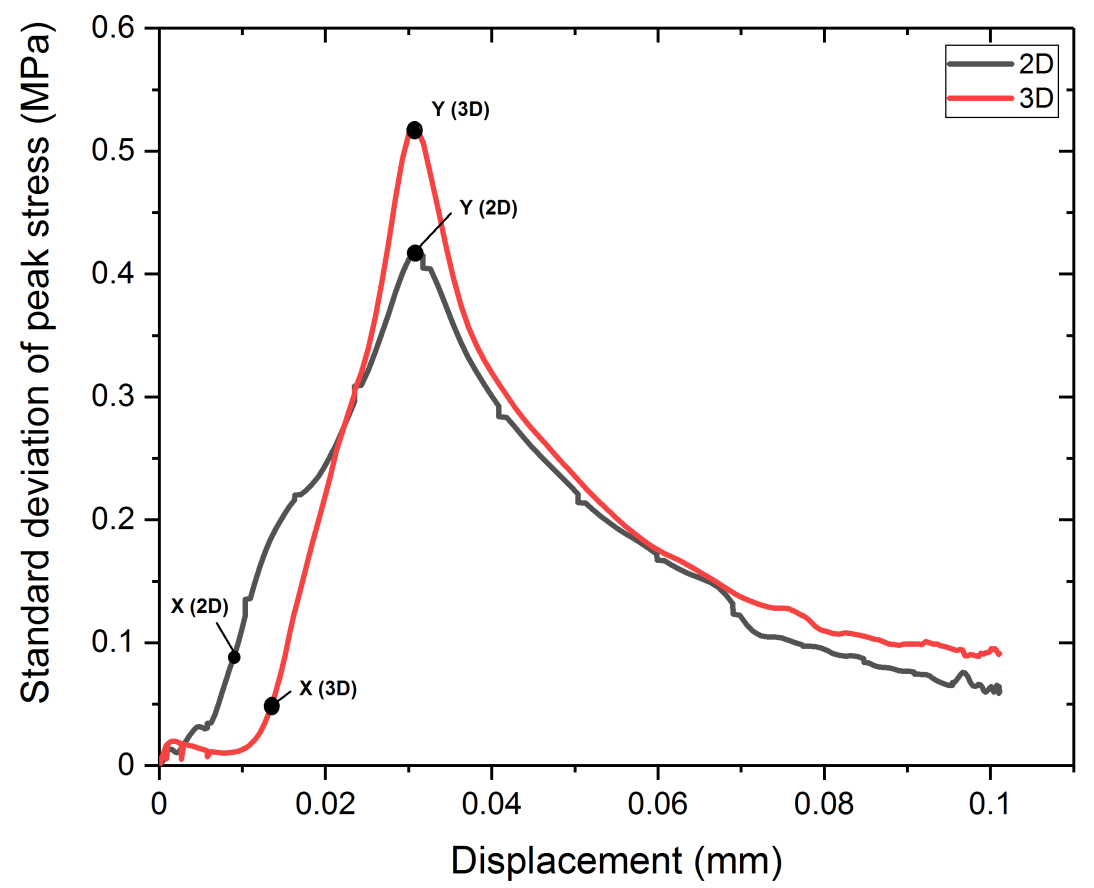

Figure 6.11: Variation of standard deviation curves of peak stress from 2D and $3 \mathrm{D}$ samples (volume fraction of capsules $=5 \%$ )

\subsubsection{Capsules volume fraction}

In order to investigate the effect of the capsule's volume fraction from capsulesbased self-healing concrete on the load carrying capacity and the fracture dissipation energy, three different volume fractions are considered here: $2 \%, 5 \%$, and $10 \%$ while the volume fraction of aggregates was kept constant at 30\%. Fig. 6.12 shows the associated models. For each volume fraction, 50 random samples were modeled to ensure statistcal convergence. 


\section{3D Numerical Simulations Under Uniaxial Tension}

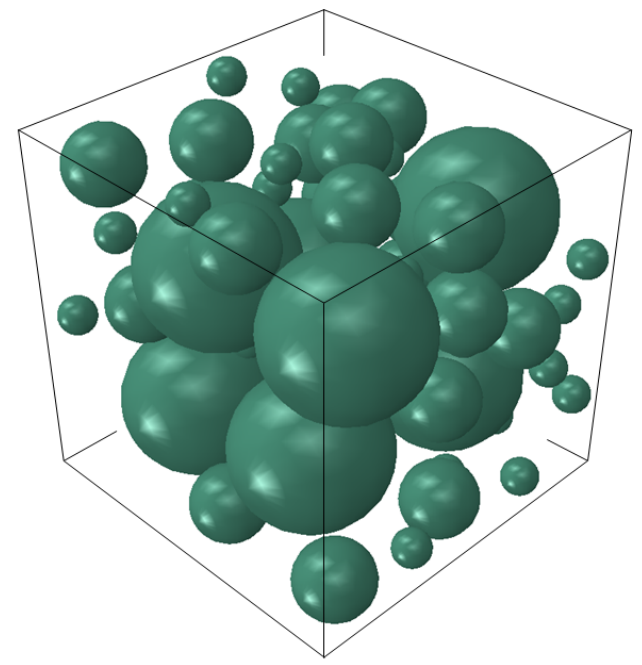

(a) Vfcap $=0 \%$

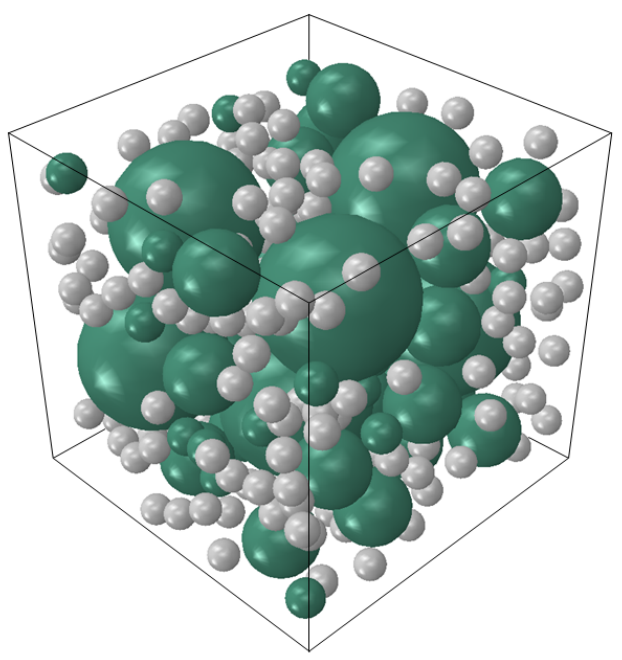

(c) Vfcap $=5 \%$

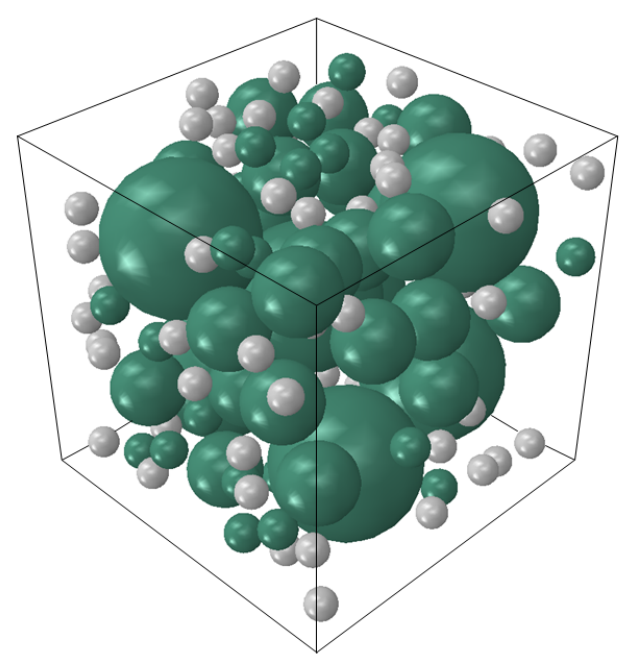

(b) Vfcap $=2 \%$

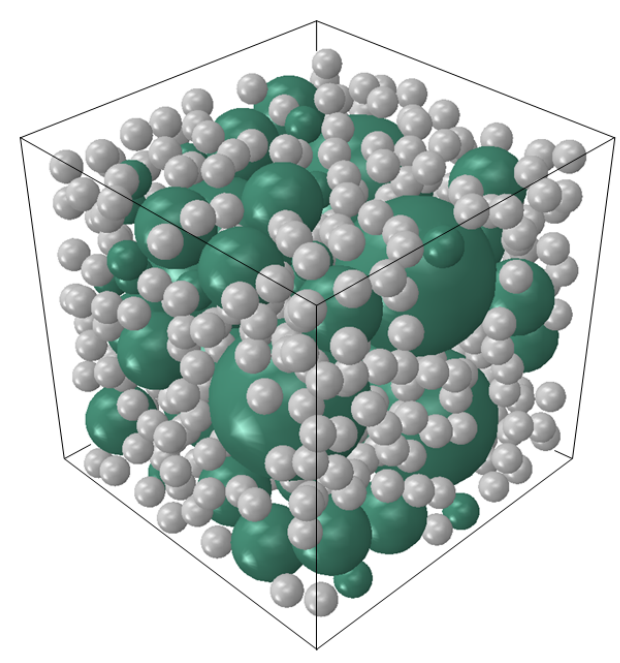

(d) Vfcap $=10 \%$

Figure 6.12: Numerical samples with different volume fraction of capsules (Vfagg $=30 \%)$

The mean stress-displacement curves can be found in Fig. 6.13 while Fig. 6.15 depicts the dissipated energy versus the displacement. Upper and lower bounds of the peak stress for 50 realizations and different capsule volume fractions are illustrated in Fig. 6.14. 


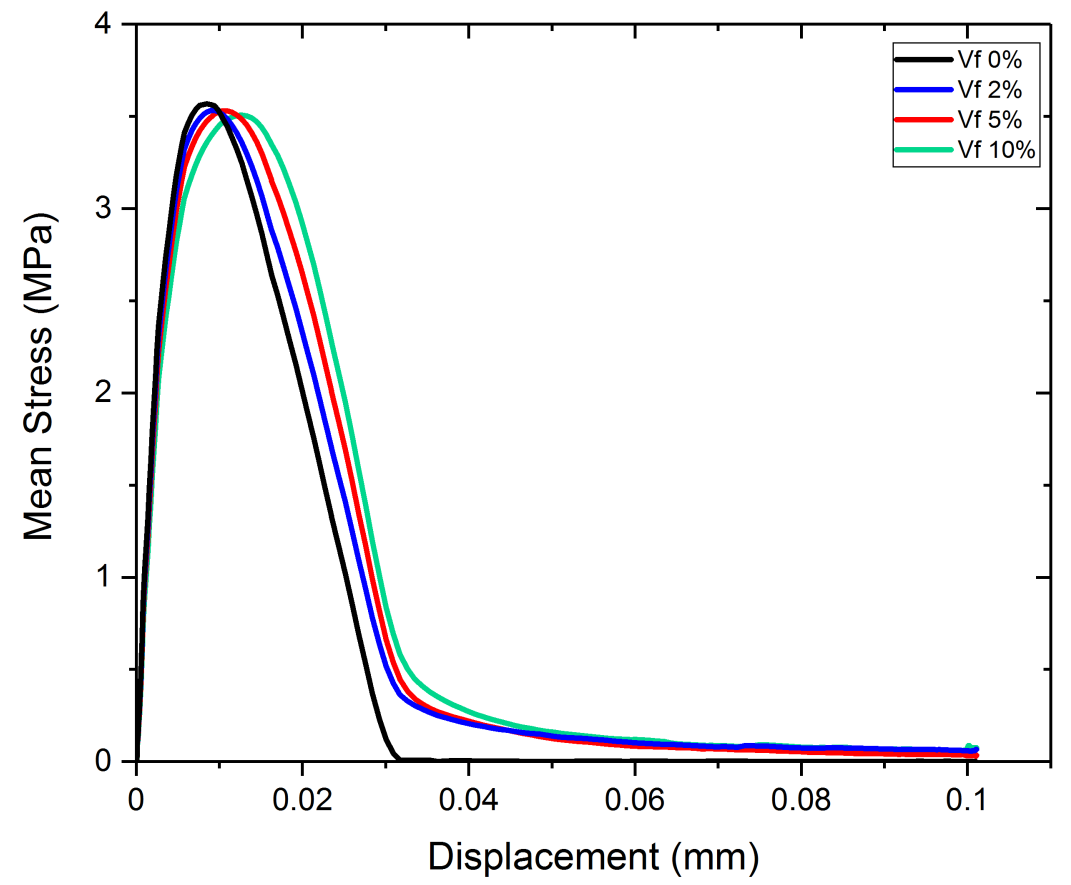

Figure 6.13: Effects of capsules volume fraction on the peak stress 
6. 3D Numerical Simulations Under Uniaxial Tension

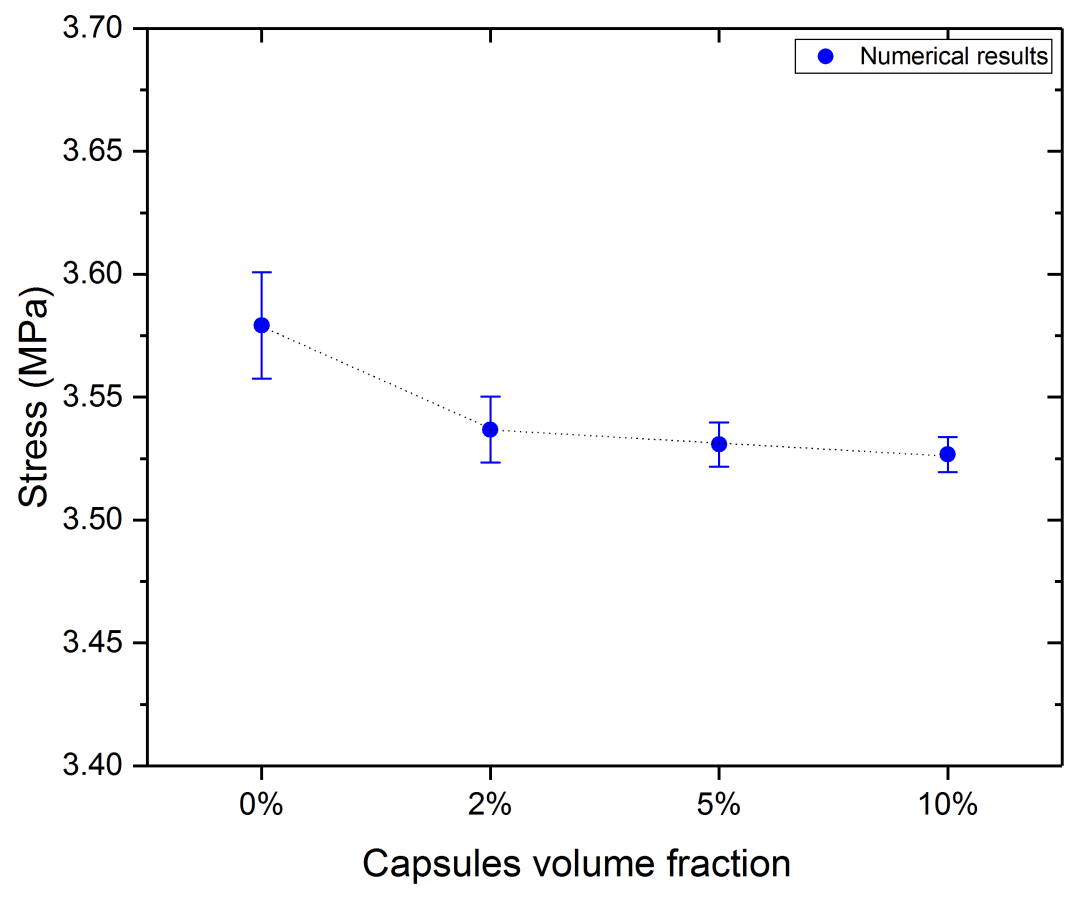

Figure 6.14: Statistical results of peak stress extracted from 50 random samples 


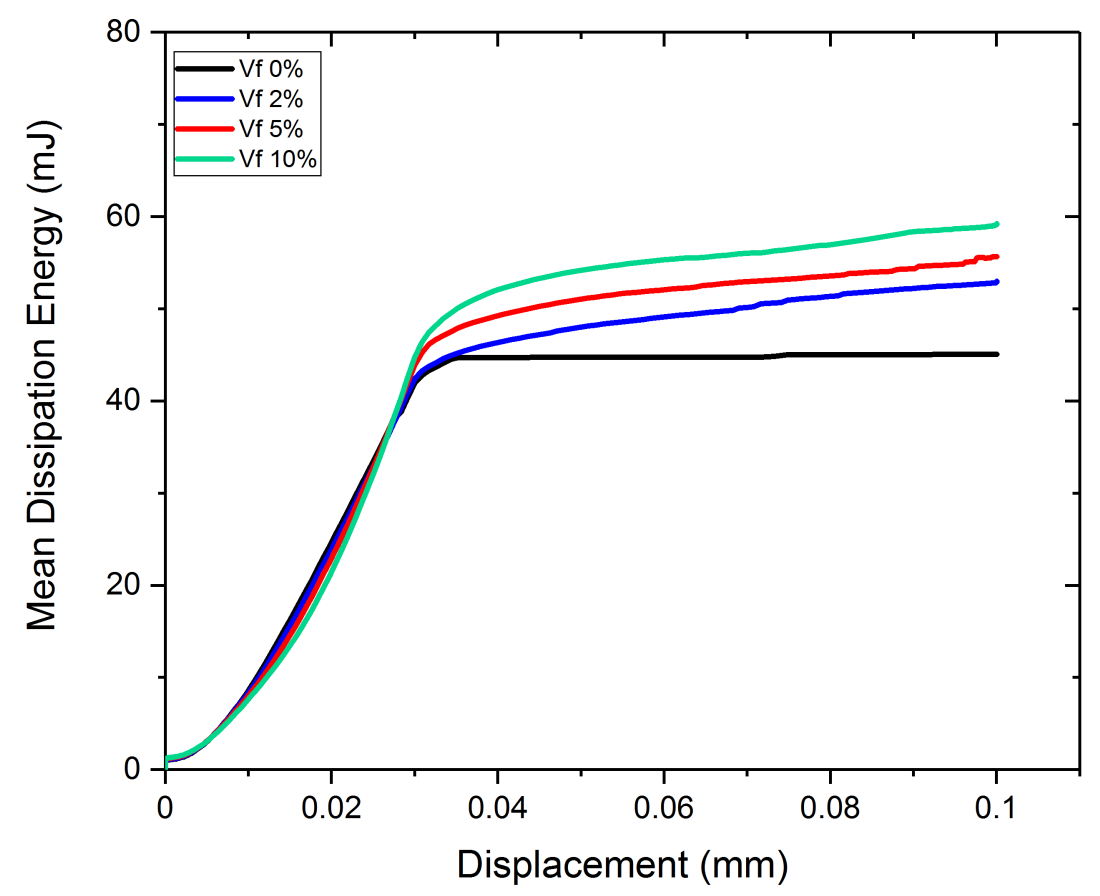

Figure 6.15: Effects of capsules volume fraction on the fracture dissipation energy

The sample without capsules has the highest mean stress around $3.58 \mathrm{MPa}$, followed by capsules with volume fraction $2 \%, 5 \%$, and $10 \%$ which has the mean stress of 3.53, 3.52, and 3.50 MPa, respectively. The higher the volume fraction of capsules added into the samples, the lower is the strength of the samples and the higher is the fracture strain. The initial elastic stiffness of the samples decrease with increasing capsule volume fraction.

The mean dissipation energy over the displacement for different capsule volume fractions - ranging from $2 \%$ to $10 \%$ - can be seen in Fig. 6.15. The dissipated energy increases with increasing capsule volume fraction. The dissipated energy increases by $13.2 \%$ (from $53 \mathrm{~mJ}$ to $60 \mathrm{~mJ}$ ) when the capsule volume fraction is increased by $8 \%$. The higher micro-cracks density indicates that more dissipation energy is needed when more capsules are embedded in the samples.

These simulation results are in good agreement with recent experimental studies with different types of microcapsules, such as the ones by Wang et al. [2014], 


\section{3D Numerical Simulations Under Uniaxial Tension}

Kanellopoulos et al. [2016], and Lv et al. [2016] using micro-encapsulated bacterial spores, pig-gelatine microcapsules carrying sodium silicate, and poly (phenolformaldehyde-PF) microcapsules, respectively. They showed that there is significant decrease in the strength in capsule-based materials compared to the original one. Note also that the capsules-based models are more ductile when more capsules are added into the samples (Fig. 6.13). Similar observations were reported in recent experimental studies [Lv et al., 2016].

\subsubsection{Rule of mixtures (ROM)}

Rule of mixtures is used in this study to compare the predicted range of tensile strength obtained from numerical simulations with the one calculated using this method. Rule of mixtures is a simple approach to approximate the various properties of composite materials based on the average of weighed volume of the phases properties. The rule of mixtures tends to overestimate the tensile strength of FRP rebars [You et al., 2017]. The tensile strength of capsule-based self-healing concrete can be predicted by a linear combination from tensile properties of matrix (m), aggregates (a), and capsules (c) as follows:

$$
\sigma_{C S H C}=\sigma_{m} V_{m}+\sigma_{a} V_{a}+\sigma_{c} V_{c}
$$

where $\sigma_{C S H C}, \sigma_{m}, \sigma_{a}$, and $\sigma_{c}$ indicate the tensile strength of capsule-based self-healing concrete, the mortar matrix, aggregates, and capsules, respectively (MPa); $V_{m}, V_{a}$, and $V_{c}$ are the associated volume fractions, respectively (\%). According to the Eq. 6.6, substituting the volume fractions of capsules ranging from $2 \%, 5 \%, 10 \%$ and tensile properties from table 4.1, the predicted tensile strengths of capsule-based self-healing concrete are $3.14 \mathrm{MPa}$, 3.35 MPa, and 3.7 MPa, respectively. These ROM predictions are in good agreement with the numerical results. 


\subsection{Fracture morphology}

Fig. 6.16 shows the microcrack evolution during the loading stages. The red color indicates the 'active' (opened) cohesive elements. Note that the deformations are magnified with a factor of 15 to better visualize the microcracks. In most cases, the final fracture pattern is dominated by a single main macrocrack which evolved from coalescence of the microcracks between two closest and largest aggregates at the top and the bottom of the samples. The fracture evolution inside the 3D specimen is presented in Fig. 6.17. At an early loading stage, a number of microcracks nucleate at the interfaces between the aggregates and mortar matrix. Some of them are located near the capsules, as can be seen in Fig. 6.17(a). With increasing loading, the microcracks grow continuously - mainly between two closest and largest aggregates - before forming a macroscopic crack. Some of the micro-cracks grow towards the capsules breaking their shells as indicated in Fig. 6.17(b). The cohesive elements shown in red colour in Fig. 6.16 and Fig. 6.17 indicate a damage value of $D \geq 0.95 ; D=1$ is complete failure. The fracture surface of the final model is visualized in Fig. 6.18; in this plot the cohesive elements are removed. 


\section{3D Numerical Simulations Under Uniaxial Tension}

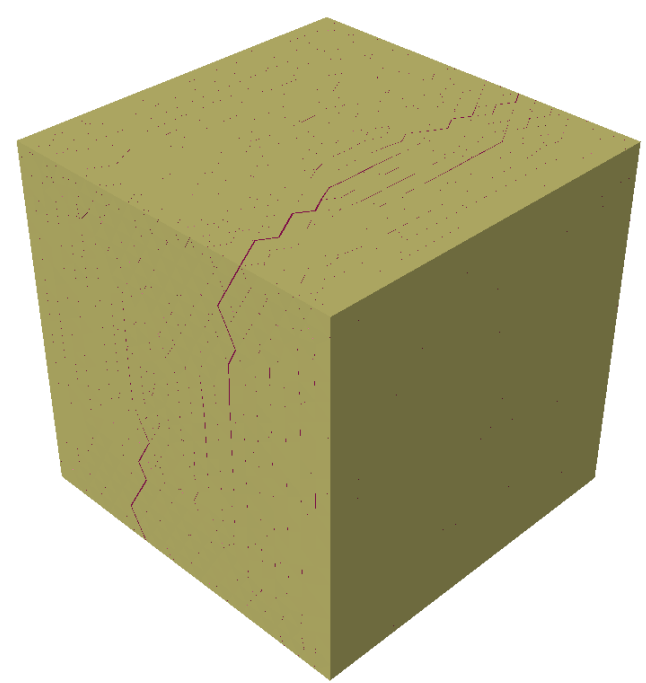

(a)

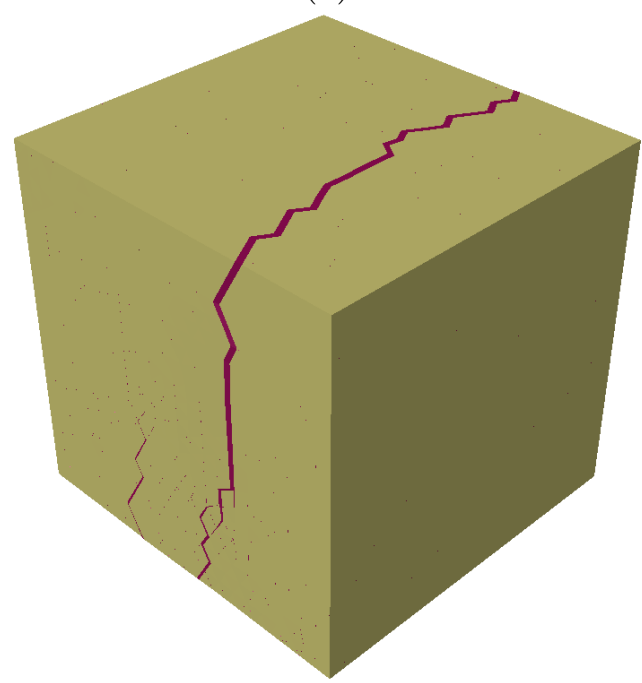

(c)

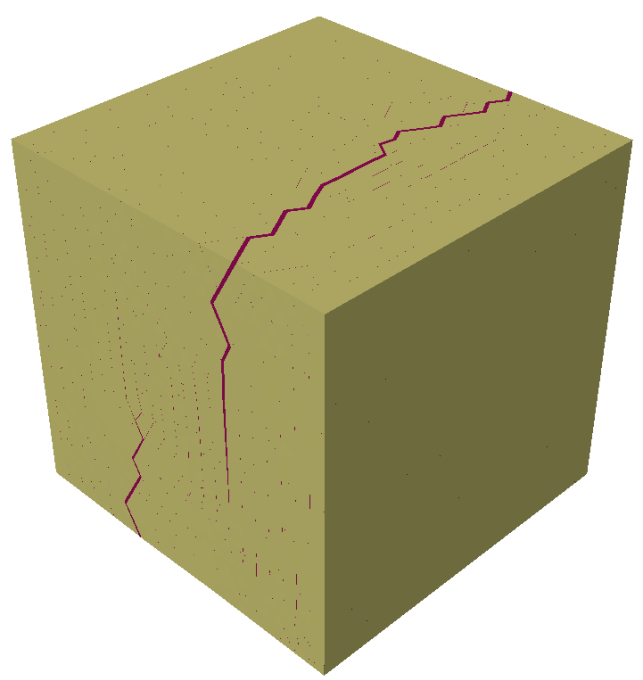

(b)

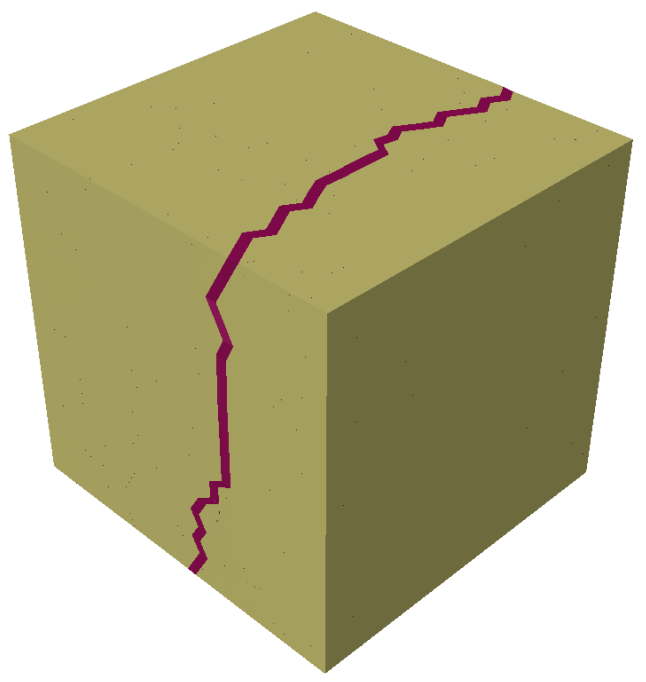

(d)

Figure 6.16: Typical type of fracture evolutions from 3D samples with corresponding displacements (volume fraction of capsule $=10 \%$ ), (a) $\mathrm{d}=0.002 \mathrm{~mm}$. (b) $\mathrm{d}=0.005 \mathrm{~mm}$. (c) $\mathrm{d}=0.01 \mathrm{~mm}$. (d) $\mathrm{d}=0.0175 \mathrm{~mm}$. 


\section{3D Numerical Simulations Under Uniaxial Tension}

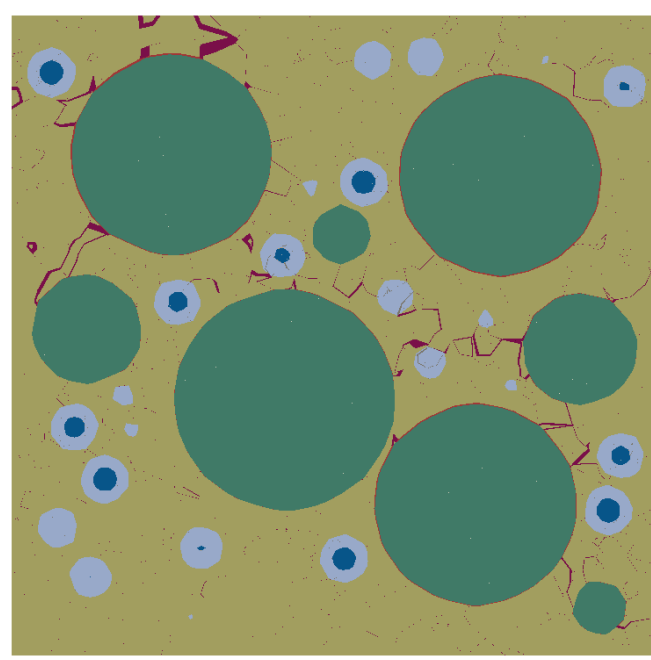

(a)

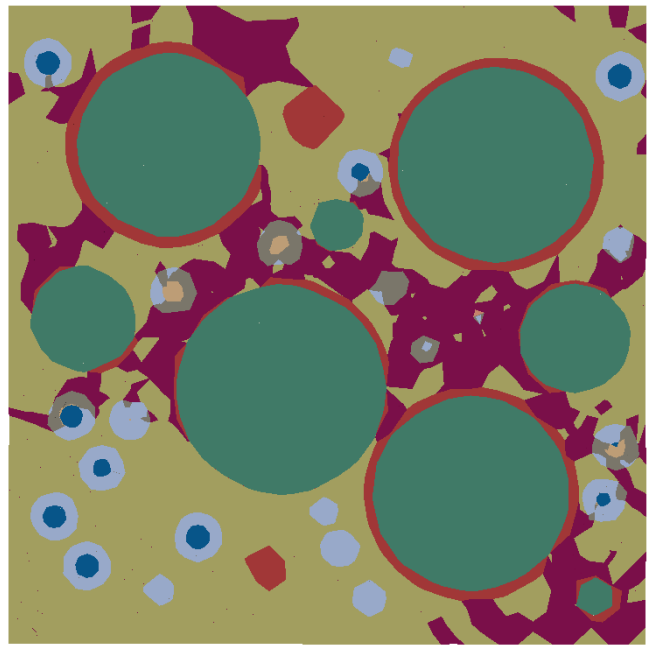

(c)

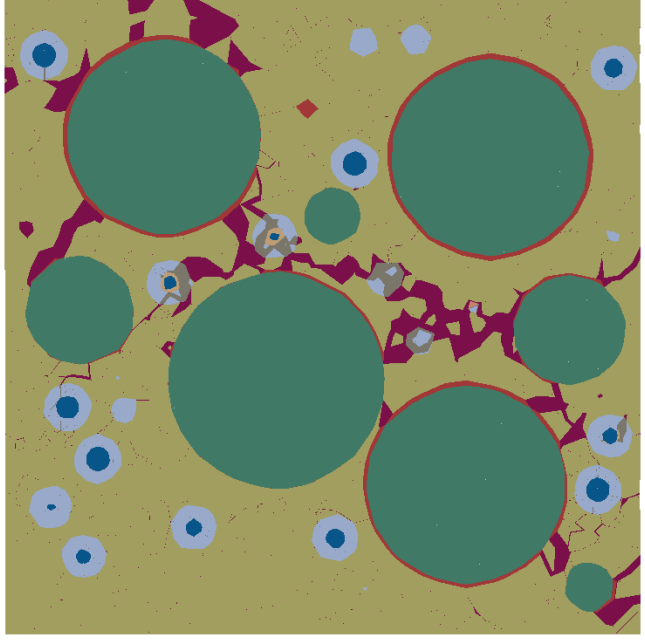

(b)

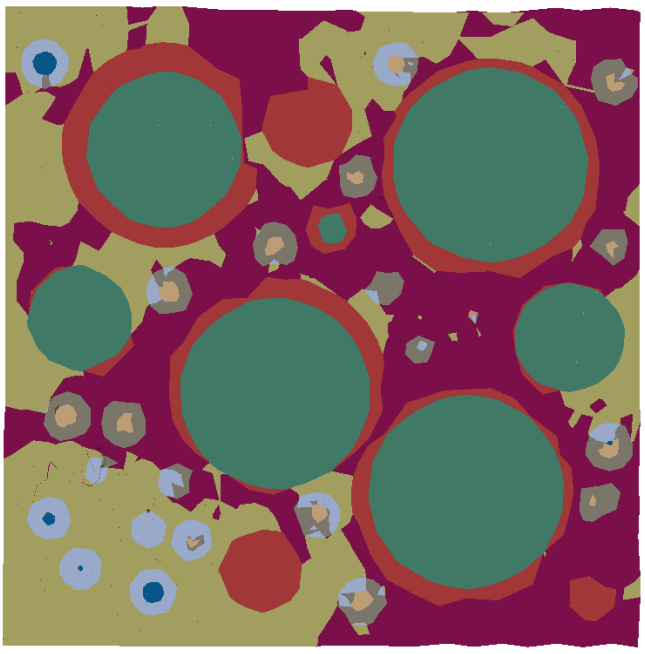

(d)

Figure 6.17: Cutting view of cracking surface evolutions from 3D specimen (volume fraction of capsules $=10 \%$ ) 


\section{3D Numerical Simulations Under Uniaxial Tension}
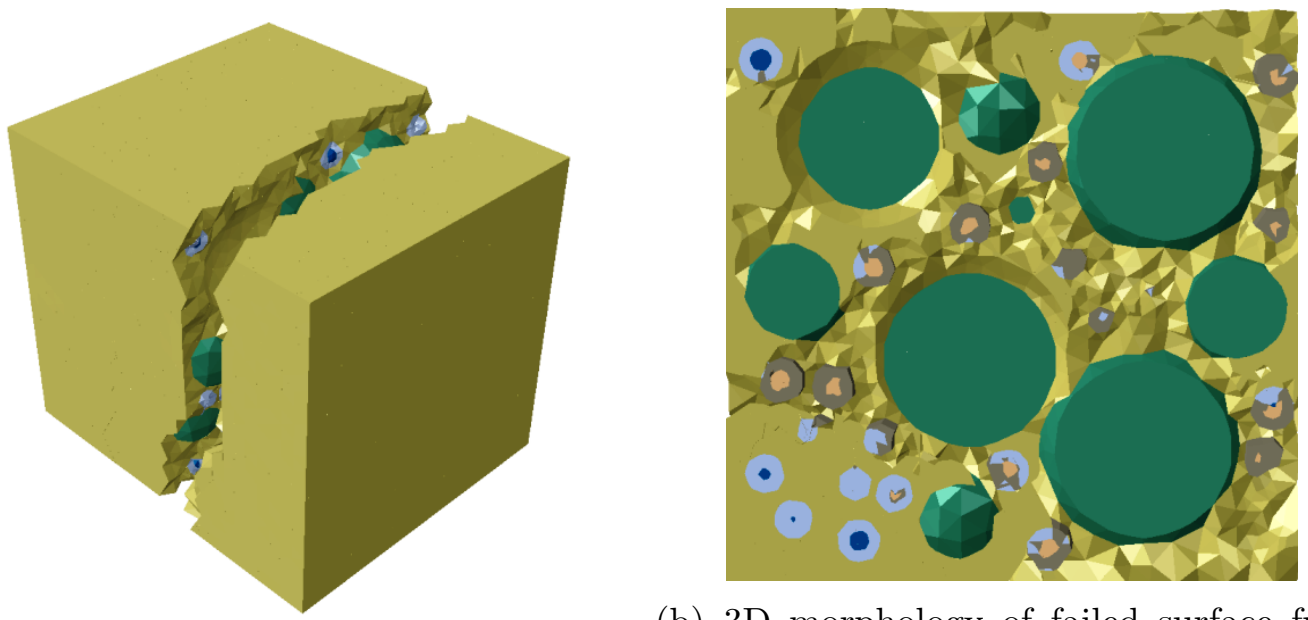

(b) 3D morphology of failed surface from (a) 3D fracture without cohesive elements cutting view model

Figure 6.18: 3D failure model of capsule-based self-healing concrete under uniaxial tension

The breakage of the capsule wall caused by an incoming crack at an early stage is crucial in self-healing concrete. Once the capsule wall is broken, the healing liquid inside the capsule will flow into the damaged area and seal the crack to restore its capacity. Fig. 6.19 shows the fracture of the capsule walls. The red cohesive elements on the capsule walls represent a damage index higher than 0.95 . 


\section{3D Numerical Simulations Under Uniaxial Tension}
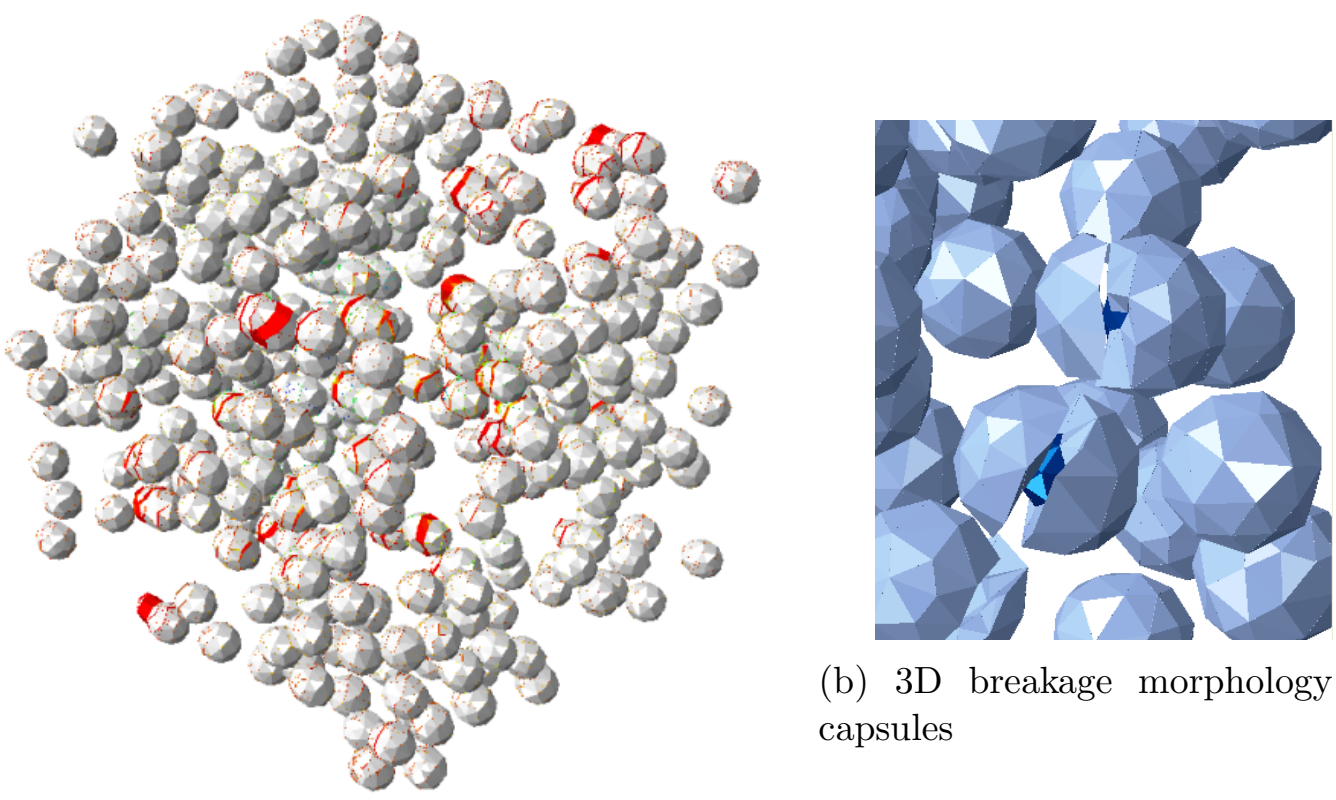

(b) 3D breakage morphology of capsules

(a) 3D damaged cohesive elements on capsule walls

Figure 6.19: Breakage of capsules in tension (only capsules are shown without aggregates and mortar matrix)

The distinct difference in the more complex fracture pattern between $2 \mathrm{D}$ and $3 \mathrm{D}$ models discussed in the previous section is the reason behind the discrepancies in the dissipated energy and load-deflection curves. 3D samples presumably predict the fracture patterns more realistically, especially in the thickness direction compared to $2 \mathrm{D}$ samples. Hence, these simulation results emphasize the importance of 3D modeling of self-healing concrete at the meso-scale. 


\section{Chapter 7}

\section{Conclusions}

This dissertation has developed computational modeling to simulate fracture in encapsulation-based self-healing concrete both of 2D and 3D models. Self-healing concrete is modeled as a multi-phases composite composed of aggregate particles, mortar, capsules core, capsules wall, and interfaces between them at the mesoscale level. Aggregates and capsules have been randomly generated using Abaqus combined with Python programming to represent the embedded microstructures inside mortar matrix materials. In order to simulate the interaction between these constituents and to account for fracture, pre-inserted cohesive elements (CIEs) with tension and shear softening constitutive laws are adopted. The extensive numerical simulations are applied to investigate the complex fracture process and to identify factors that affect capsules being fractured in encapsulation-based selfhealing concrete under uniaxial tension. The effects of volume fraction of capsules on the load capacity, fracture energy, and the probability of fractured capsule are assessed. The relationship of interfacial strength of capsule with capsule thickness on the crack propagation are identified. The fracture criteria based on combination in elastic and fracture properties of the capsule are highlighted. Finally, the reliability of $2 \mathrm{D}$ and $3 \mathrm{D}$ models of fracture in encapsulation-based self-healing concrete at mesoscale level are compared. Based on numerical studies that have been conducted in this dissertation, the following conclusions can be summarized as follows:

1. The results of this study revealed that volume fraction of capsules has a significant effect on the macroscopic behavior of self-healing concrete. 
An increased volume fraction of capsules from $1.57 \%$ to $9.42 \%$ reduces the strength by $6.97 \%$ and increases the dissipation energy by $13.66 \%$.

2. Increasing volume fraction of capsules from $1.57 \%$ to $9.42 \%$ led to increase the percentage of fractured capsules from $13.5 \%$ to $92.3 \%$ (Ratio 15:1), 9.8\% to $72.3 \%$ (Ratio $10: 1$ ), $1.5 \%$ to $16.9 \%$ (Ratio $5: 1$ ), and $0 \%$ to $2.9 \%$ (Ratio $1: 1)$.

3. The volume fraction of the capsules in the range of $0-2 \%$ has no significant effect on the macroscopic behavior of self-healing concrete.

4. The increasing strength of self-healing concrete remain linear when the interfacial strength (itz) of capsule is ranging from $0 \%-50 \%$ of the strength of mortar matrix.

5. The fractured capsule occurs when using softer capsule (e.g., Ec/Em =1/7) compared to the one with stiffer capsule (e.g., Ec/Em = 2).

6. The 3D modeling has a pronounced influence not only on macroscopic mechanical properties but also on the crack patterns compared to associated 2D models. Extending the fracture model from 2D to 3D, increases the mean ultimate stress and dissipation energy by $27.3 \%$ and $38.6 \%$, respectively.

The sensitivity analysis of modeling parameters used in the simulations has not been yet investigated in this dissertation and should be performed in the future for better results and understanding the modeling limitations.

Despite of that limitation, the proposed method performed in this dissertation has shown the efficient results to predict fracture behavior in encapsulation-based self-healing concrete. The output from this dissertation will be valuable input for engineering, material sciences, and particular manufacturer's company as well in designing an appropriate capsule materials for capsules-based self-healing concrete. 


\section{Appendix}

Python code for 3D microstructures generation in encapsulation-based self-healing concrete at mesoscale level. It is developed using Python 3.4 and should be used along with Abaqus CAE v.6.12.

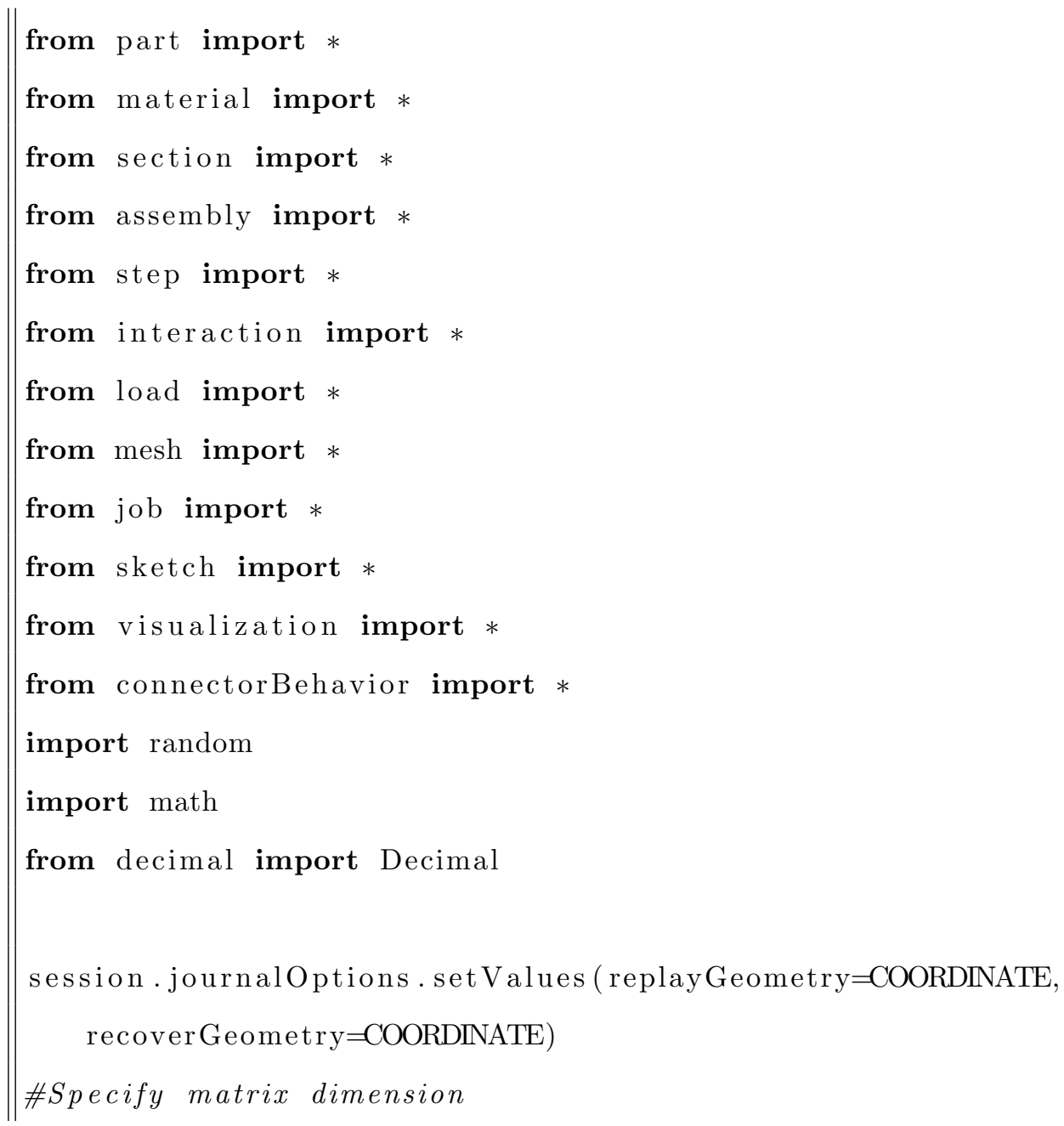




$$
\begin{aligned}
& \mathrm{w}=25.0 \\
& \mathrm{~h}=25.0 \\
& \mathrm{l}=25.0
\end{aligned}
$$

\section{type $=$ DEFORMABLEBODY)}

mdb. models ['Model-1'] . parts ['matrix']. BaseSolidExtrude (depth=l , sketch $=$ mdb. models ['Model-1']. sketches [' _- profile ${ }_{--}$']) del mdb. models['Model-1']. sketches [' _-profile _-']

\#aggregate particle distribution according to Hirsch \#define sieve opening size limits for different classes $\mathrm{dClass} \operatorname{Min}=[]$ $\mathrm{dClassMax}=[]$

dClassMin .append (16.00) \#1

dClassMax . append (16.00)

dClassMax . append (12.70) \#2

dClass Min . append (12.70)

dClassMax . append $(9.50) \# 3$

$\mathrm{dClassMin}$. append (9.50)

dClassMax . append ( 4.75$) \# 4$

dClass Min . append (4.75)

dClassMax . append (2.36) \#5 


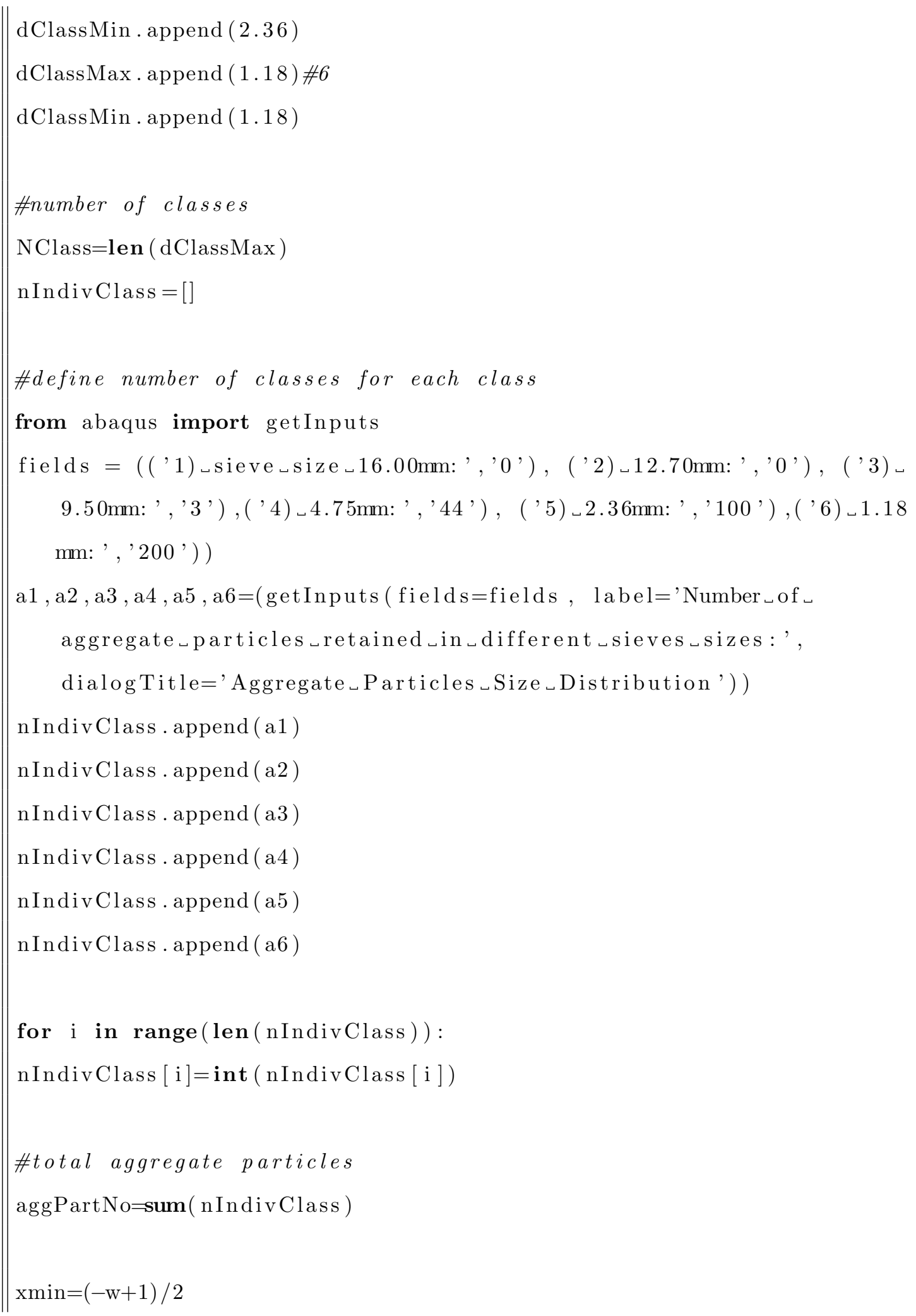




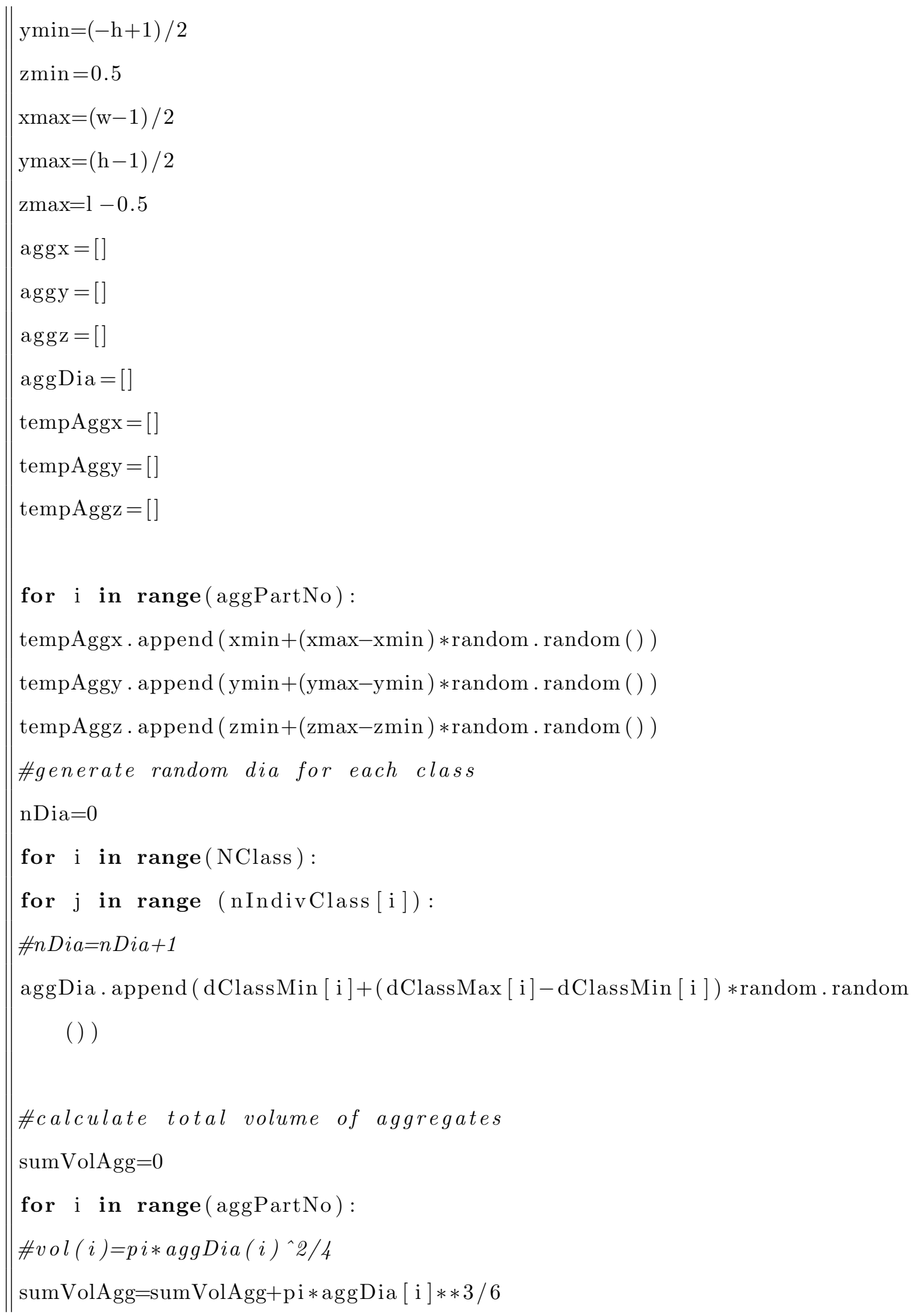


\#to find the distances of aggregate particles

def borderCut $(\mathrm{x}, \mathrm{y}, \mathrm{z}, \mathrm{d})$ :

left Check=math. $\operatorname{sqrt}((\mathrm{x}-\mathrm{xmin}) * * 2)$

rightCheck $=$ math. sqrt $((\mathrm{x}-\mathrm{xmax}) * * 2)$

bottom Check=math. sqrt $((\mathrm{y}-\mathrm{ymin}) * * 2)$

topCheck=math. sqrt $((\mathrm{y}-\mathrm{ymax}) * * 2)$

frontCheck=math. $\operatorname{sqrt}((\mathrm{z}-\mathrm{zmin}) * * 2)$

backCheck=math. sqrt $((\mathrm{z}-\mathrm{zmax}) * * 2)$

if $\operatorname{leftCheck}<=\mathrm{d} / 2$ or $\operatorname{rightCheck}<=\mathrm{d} / 2$ or bottomCheck $<=\mathrm{d} / 2$ or topCheck $<=\mathrm{d} / 2$ or frontCheck $<=\mathrm{d} / 2$ or backCheck $<=\mathrm{d} / 2$ :

return 1

else :

return 0

\#creating ismember function

def ismember $(\mathrm{A}, \mathrm{b})$ :

if $\mathrm{b}$ in $\mathrm{A}$ :

return 1

else :

return 0

def checkprev $(x, y, z, d$, temp_x_i , temp_y_i, temp_z_i $)$ :

def $\operatorname{lapmap}\left(\mathrm{x}, \mathrm{y}, \mathrm{z}, \mathrm{d}, \mathrm{temp} \mathrm{z}_{-} \mathrm{i}, \mathrm{temp} \mathrm{y}_{-} \mathrm{i}, \mathrm{temp} \mathrm{z}_{-} \mathrm{i}\right)$ :

$\operatorname{lap}=[]$

$\mathrm{N}=\operatorname{len}(\mathrm{x})$

$\mathrm{n}=\mathrm{N}$

for $i$ in $\operatorname{range}(\mathrm{N})$ : 


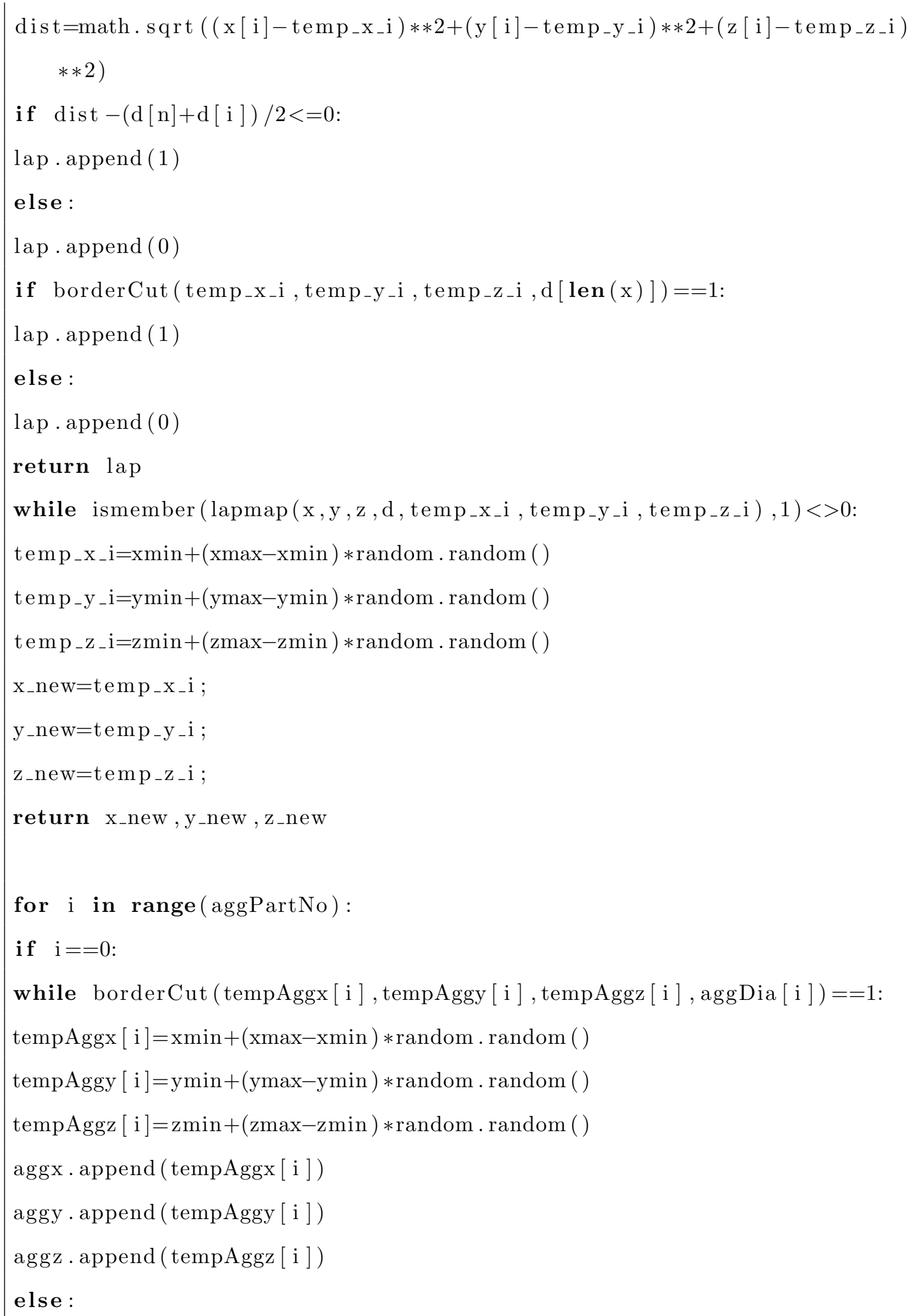




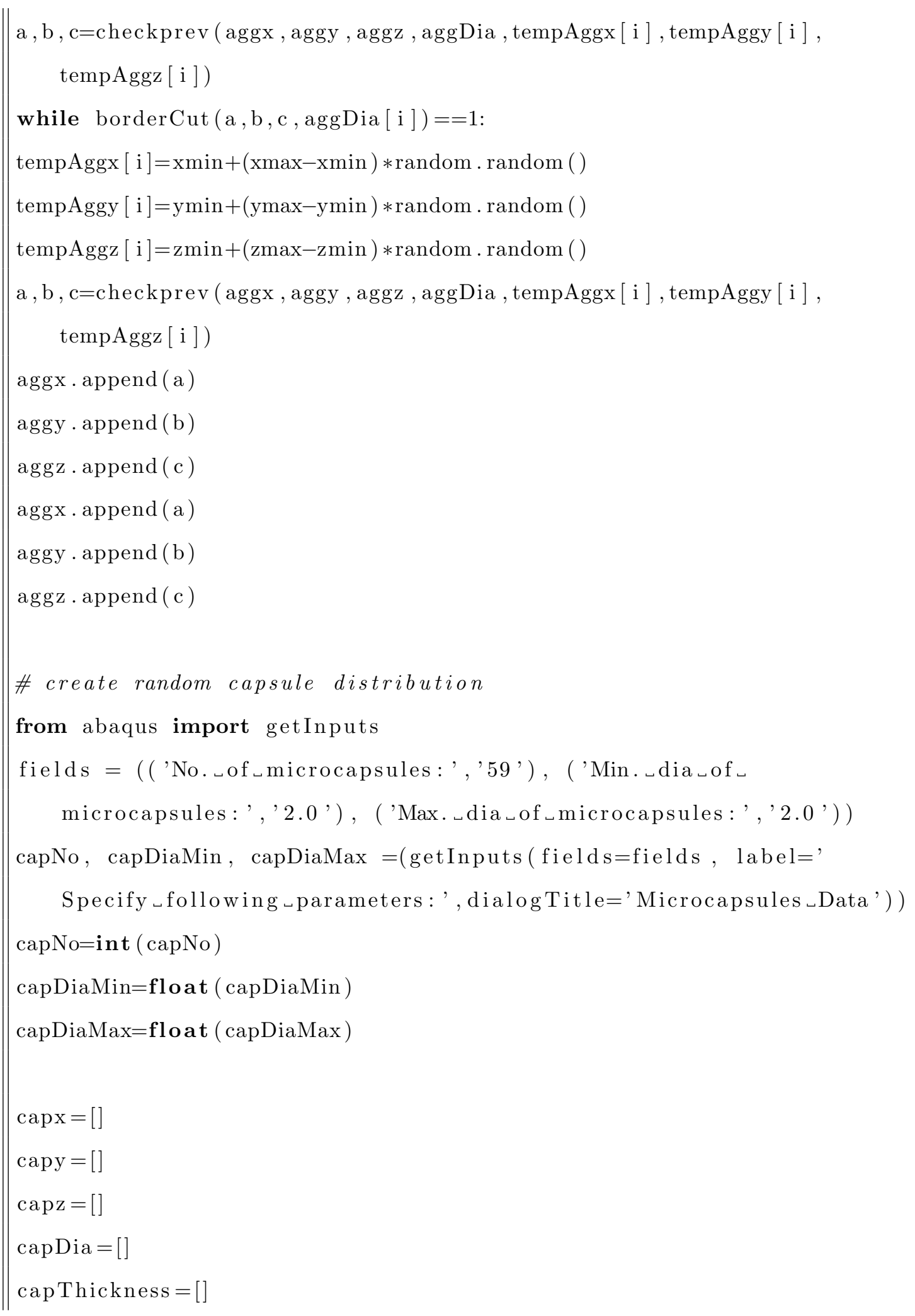


tempCapx $=[]$

tempCapy $=[]$

tempCapz $=[]$

$\operatorname{agg} \operatorname{Capx}=[]$

$\operatorname{agg}$ Capy $=[]$

$\operatorname{aggCapz}=[]$

$\operatorname{agg}$ CapDia $=[]$

for $i$ in range (capNo):

tempCapx . append $(x \operatorname{xmin}+(\operatorname{xmax}-x \min ) *$ random. $\operatorname{random}())$

tempCapy . append $(\operatorname{ymin}+(\operatorname{ymax}-\mathrm{ymin}) * \operatorname{random}$.random ()$)$

tempCapz . append $(z \min +(\operatorname{zmax}-z \min ) *$ random . random ()$)$

capDia . append (capDiaMin+(capDiaMax-capDiaMin $) *$ random . random ()$)$

capThickness . append (capDia [i]/27)

for $\mathrm{i}$ in range $(\operatorname{agg} \mathrm{PartNo})$ :

$\operatorname{agg} C \operatorname{apx} . \operatorname{append}(\operatorname{agg} x[\mathrm{i}])$

aggCapy . append (aggy [i ])

$\operatorname{aggCapz}$. append (aggz [i ] )

aggCapDia . append (aggDia [i ])

for $i$ in range (capNo):

aggCapDia. append (capDia [i ])

for $i$ in range (capNo):

a , b , c=checkprev (aggCapx , aggCapy, aggCapz, aggCapDia , tempCapx [i ] , tempCapy [i ] , tempCapz [i ])

while borderCut $(\mathrm{a}, \mathrm{b}, \mathrm{c}, \mathrm{capDia}[\mathrm{i}])==1$ :

tempCapx $[\mathrm{i}]=\mathrm{xmin}+(\mathrm{xmax}-\mathrm{xmin}) *$ random $\cdot \operatorname{random}()$

tempCapy $[\mathrm{i}]=\mathrm{ymin}+(\mathrm{ymax}-\mathrm{ymin}) * \operatorname{random} . \operatorname{random}()$ 


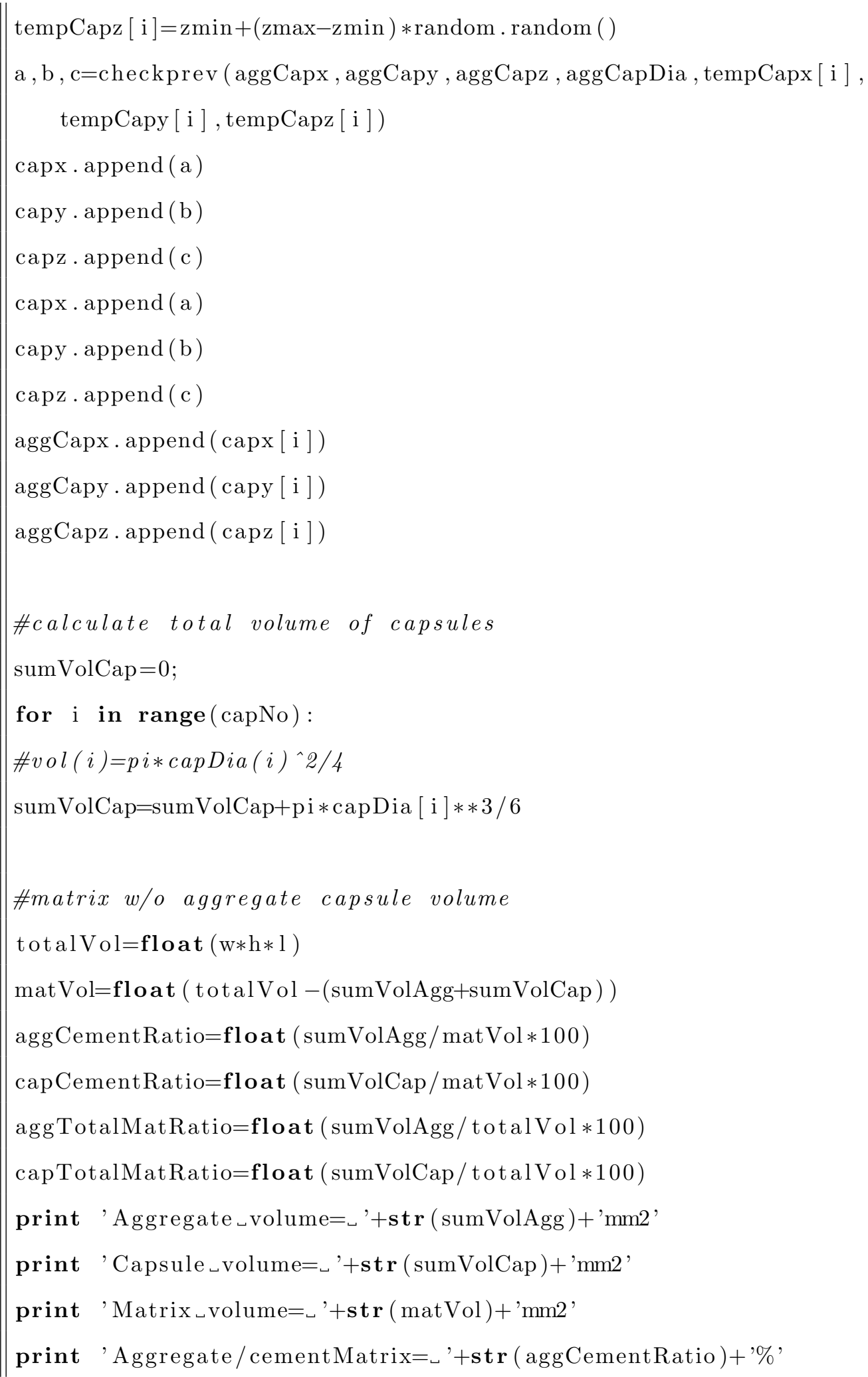




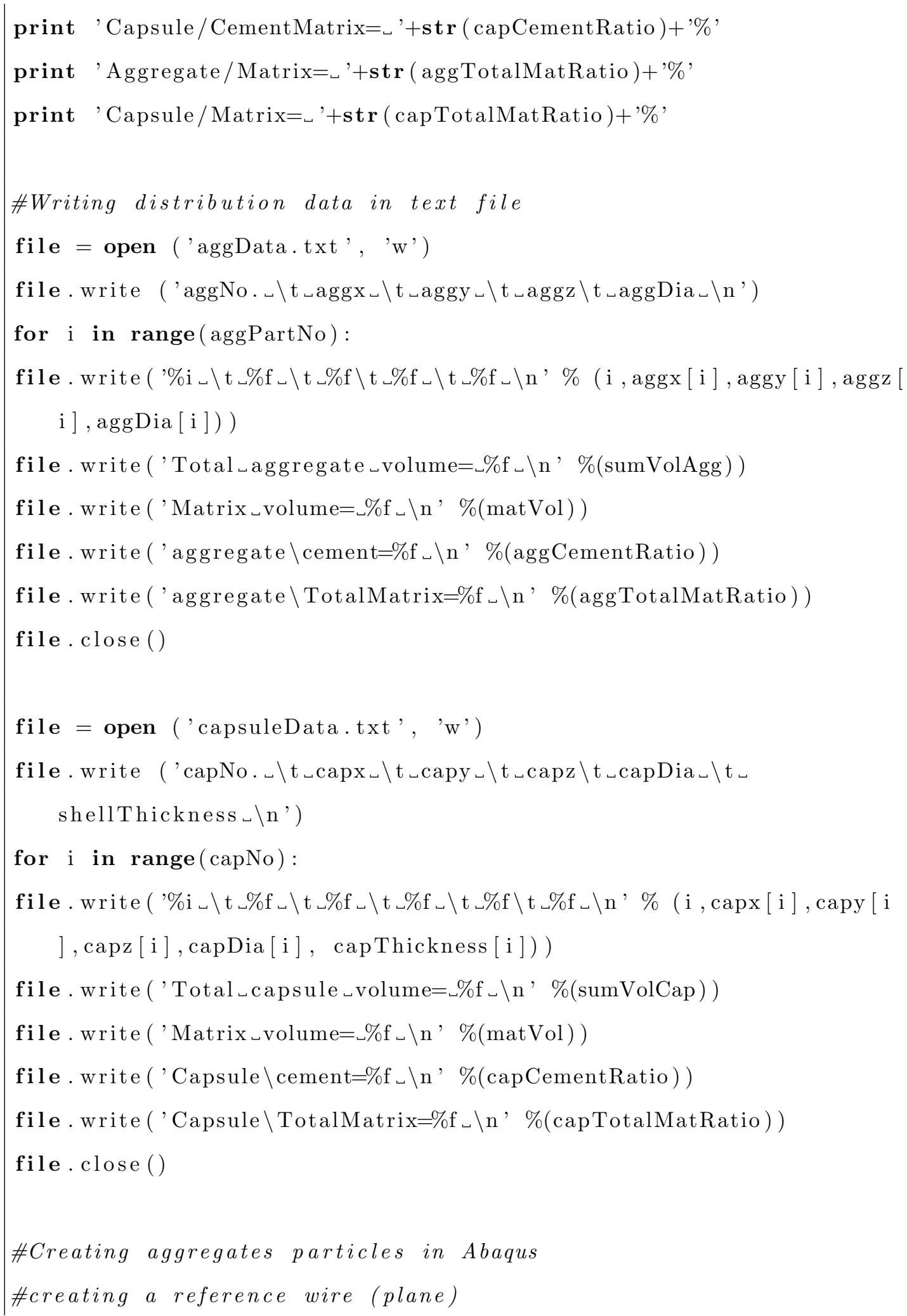




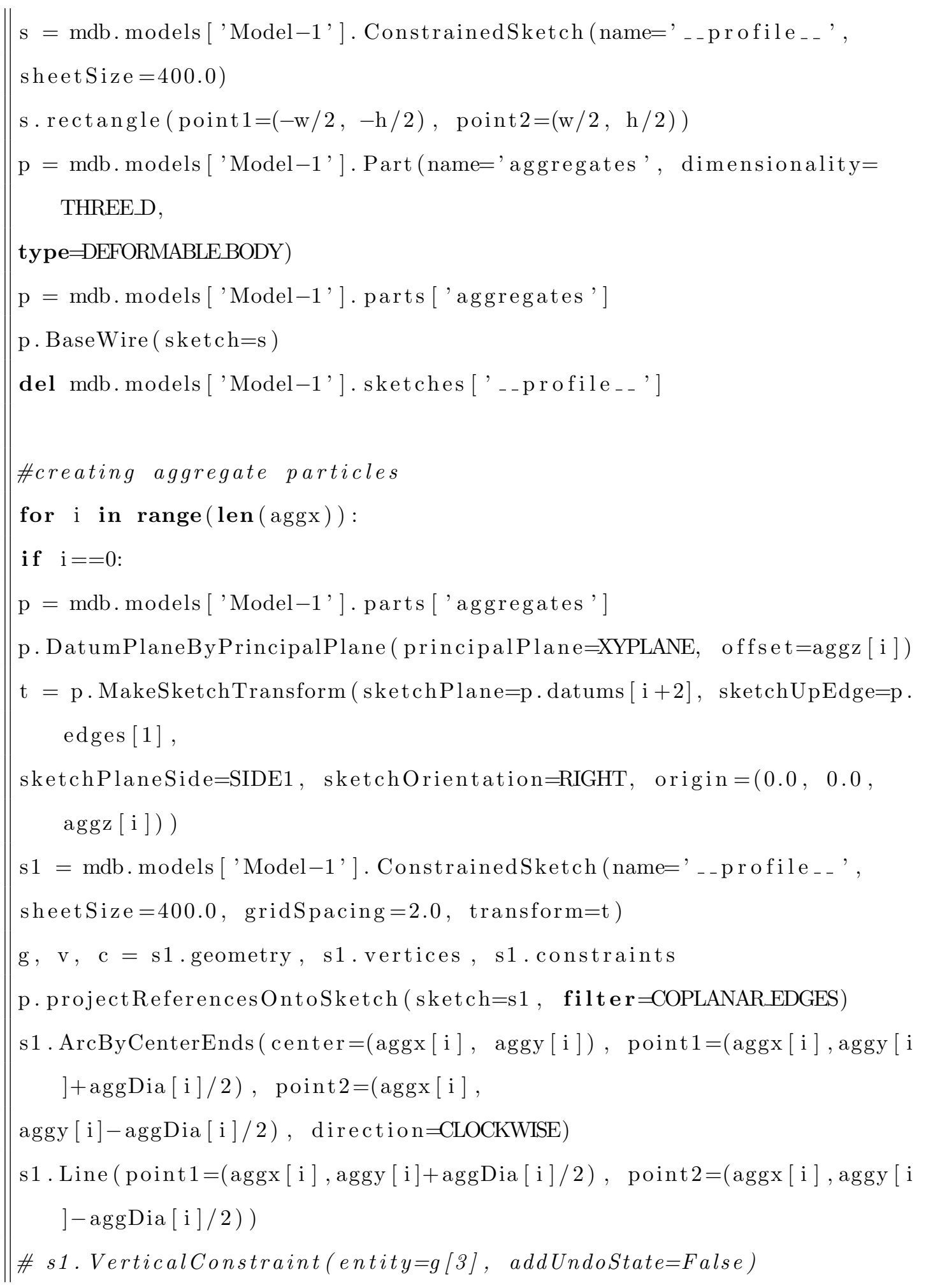




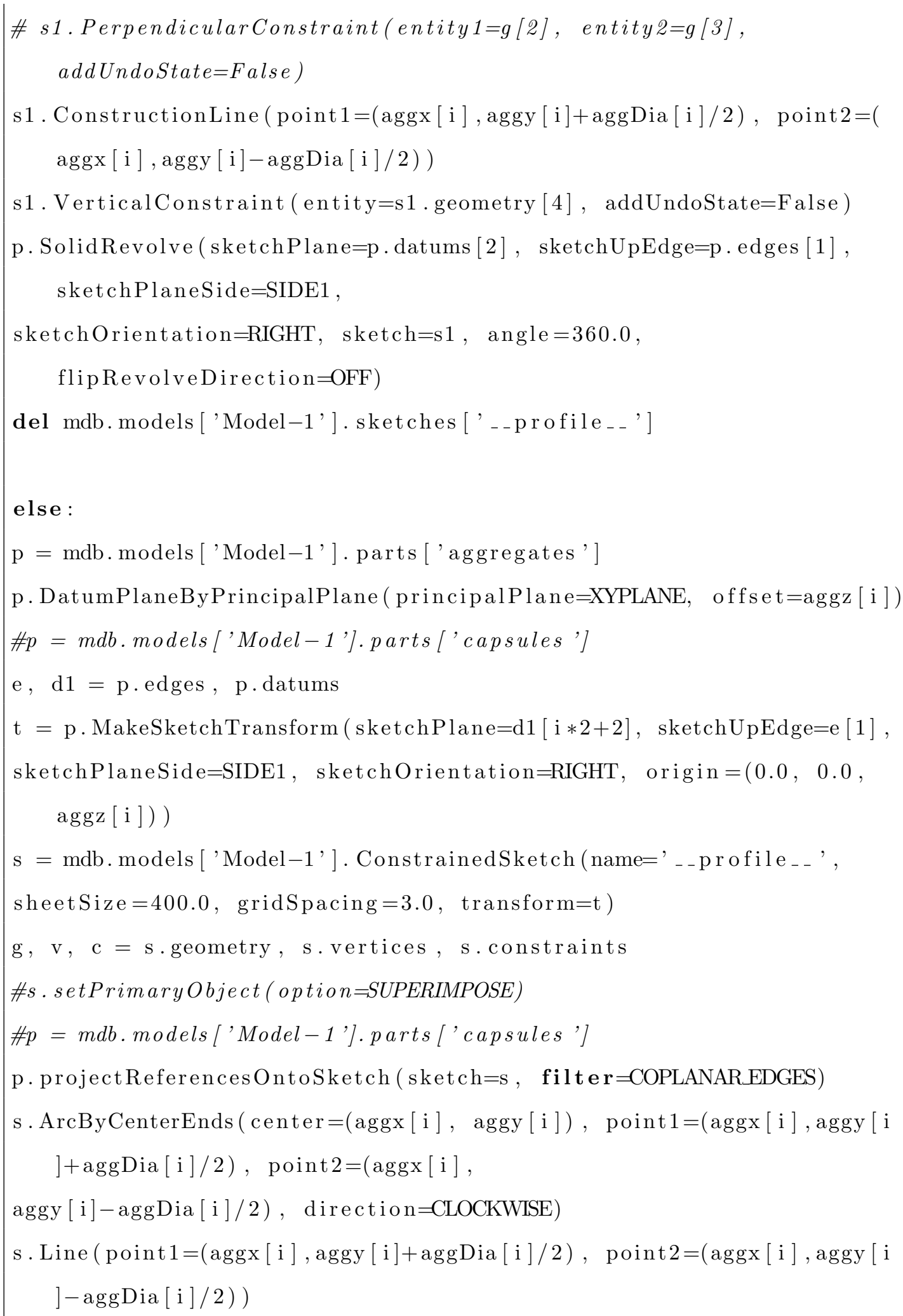




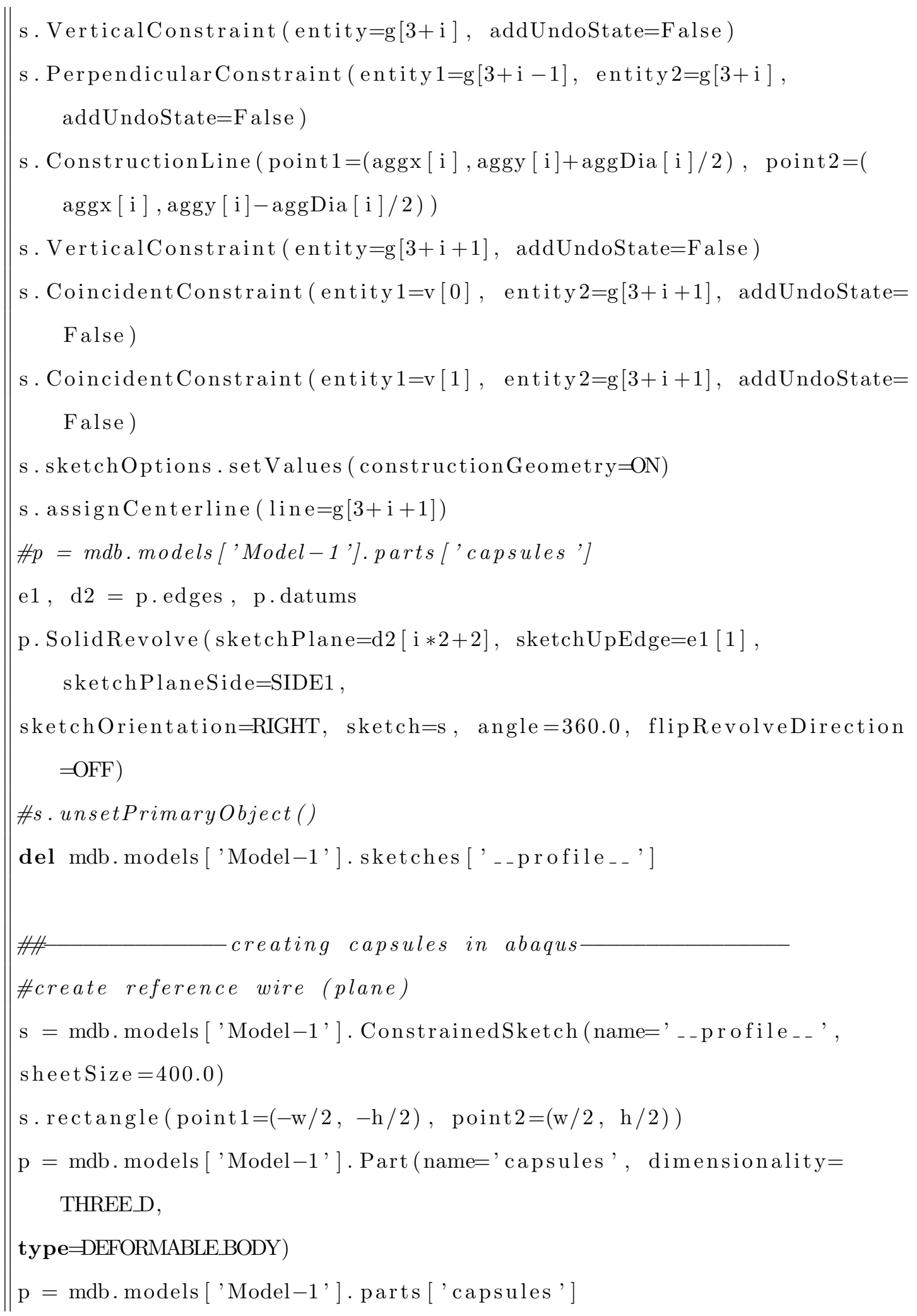




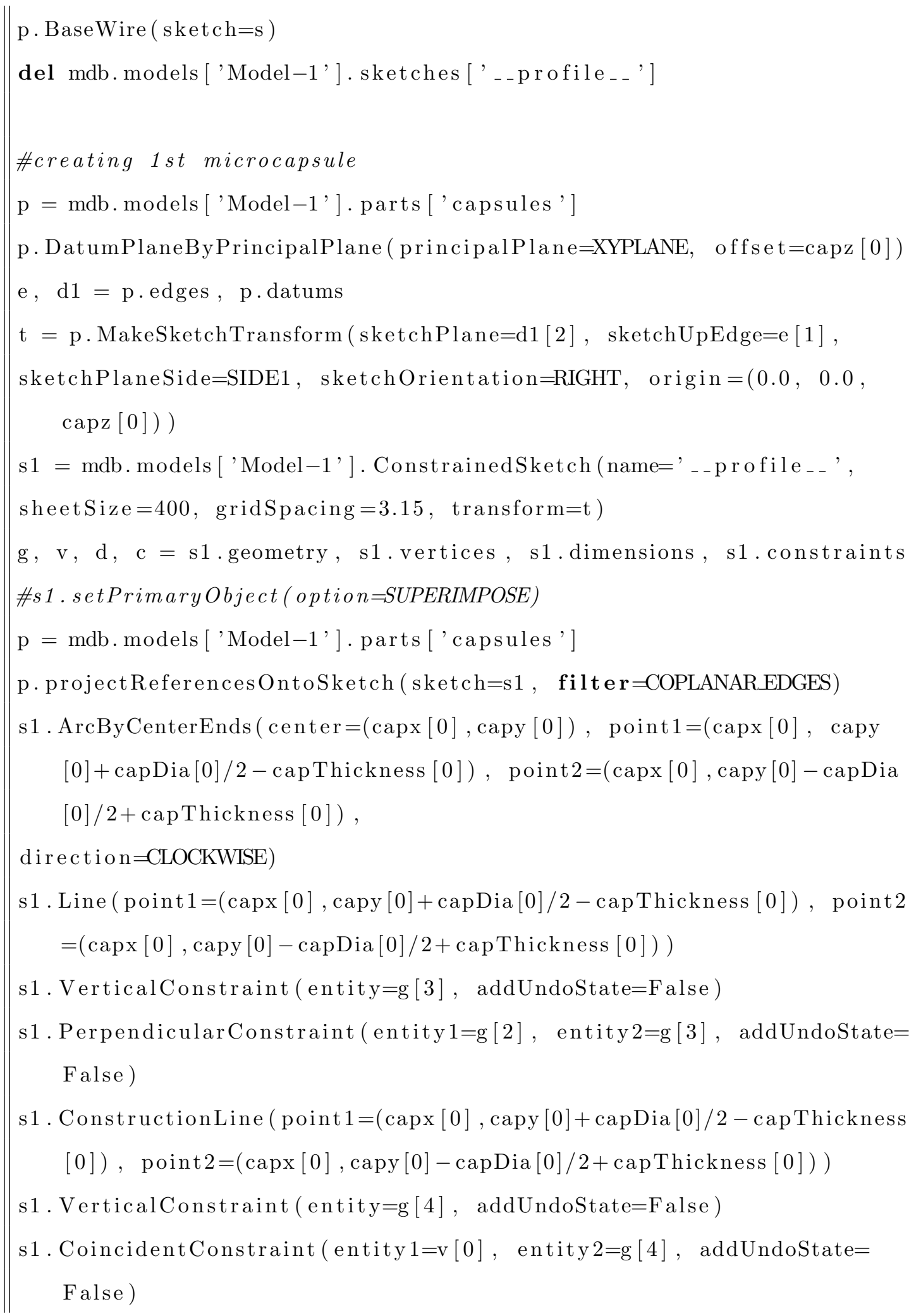


s1. CoincidentConstraint ( entity $1=\mathrm{v}[1], \quad$ entity $2=\mathrm{g}[4]$, addUndoState= False )

$\mathrm{p}=\operatorname{mdb} \cdot \operatorname{model}[$ ['Model -1 '] $\operatorname{parts}[$ 'capsules']

e1, $\mathrm{d} 2=\mathrm{p}$.edges, p.datums

p. SolidRevolve ( sketchPlane=d2 [2], sketchUpEdge=e1 [1],

sketchPlaneSide=SIDE1,

sketchOrientation=RIGHT, sketch=s1, angle $=360.0$,

flipRevolveDirection=OFF)

del mdb. models['Model-1']. sketches [' _- profile _.' ]

$\mathrm{p}=\operatorname{mdb} \cdot \operatorname{models}[$ 'Model-1'] $\operatorname{parts}[$ 'capsules']

e1, $\mathrm{d} 2=\mathrm{p}$.edges, p.datums

$\mathrm{t}=\mathrm{p}$. MakeSketchTransform $(\operatorname{sketchPlane}=\mathrm{d} 2[2], \operatorname{sketchUpEdge=e1[1],}$ sketchPlaneSide=SIDE1,

sketchOrientation=RIGHT, origin $=(0.0,0.0, \operatorname{capz}[0]))$

$\mathrm{s} 1=$ mdb. models ['Model-1']. ConstrainedSketch (name=' _-profile _- ', sheetSize $=400$, gridSpacing $=3.42$, transform $=$ t)

$\mathrm{g}, \mathrm{v}, \mathrm{d}, \mathrm{c}=\mathrm{s} 1$. geometry, s1.vertices, s1.dimensions, s1.constraints \#s1. setPrimaryObject (option=SUPERIMPOSE)

$\mathrm{p}=\operatorname{mdb} \cdot \operatorname{models}[$ 'Model-1'] $\operatorname{parts}[$ 'capsules']

p. projectReferences OntoSketch ( $\operatorname{sketch=s1，\quad filter=COPLANAREDGES)~}$

s1. ArcByCenterEnds $(\operatorname{center}=(\operatorname{capx}[0], \quad \operatorname{capy}[0]), \operatorname{point} 1=(\operatorname{capx}[0], \operatorname{capy}$ $[0]+\operatorname{capDia}[0] / 2-\operatorname{capThickness}[0]), \operatorname{point} 2=(\operatorname{capx}[0], \operatorname{capy}[0]-\operatorname{capDia}$ $[0] / 2+$ capThickness $[0])$,

dir e c t i o $=$ CLOCKWISE)

s1. ArcByCenterEnds $(\operatorname{center}=(\operatorname{capx}[0], \quad \operatorname{capy}[0]), \operatorname{point} 1=(\operatorname{capx}[0], \quad \operatorname{capy}$ $[0]+\operatorname{capDia}[0] / 2), \operatorname{point} 2=(\operatorname{capx}[0], \quad \operatorname{capy}[0]-\operatorname{capDia}[0] / 2)$, dir e cti o $=$ CLOCKWISE)

s1. CoincidentConstraint ( entity $1=\mathrm{v}$. find At ( ( $\operatorname{capx}[0], \quad$ capy $[0]+\operatorname{capDia}$ $[0] / 2))$, entity $2=$ g. findAt $((\operatorname{capx}[0]$, capy $[0]))$, addUndoState=False 


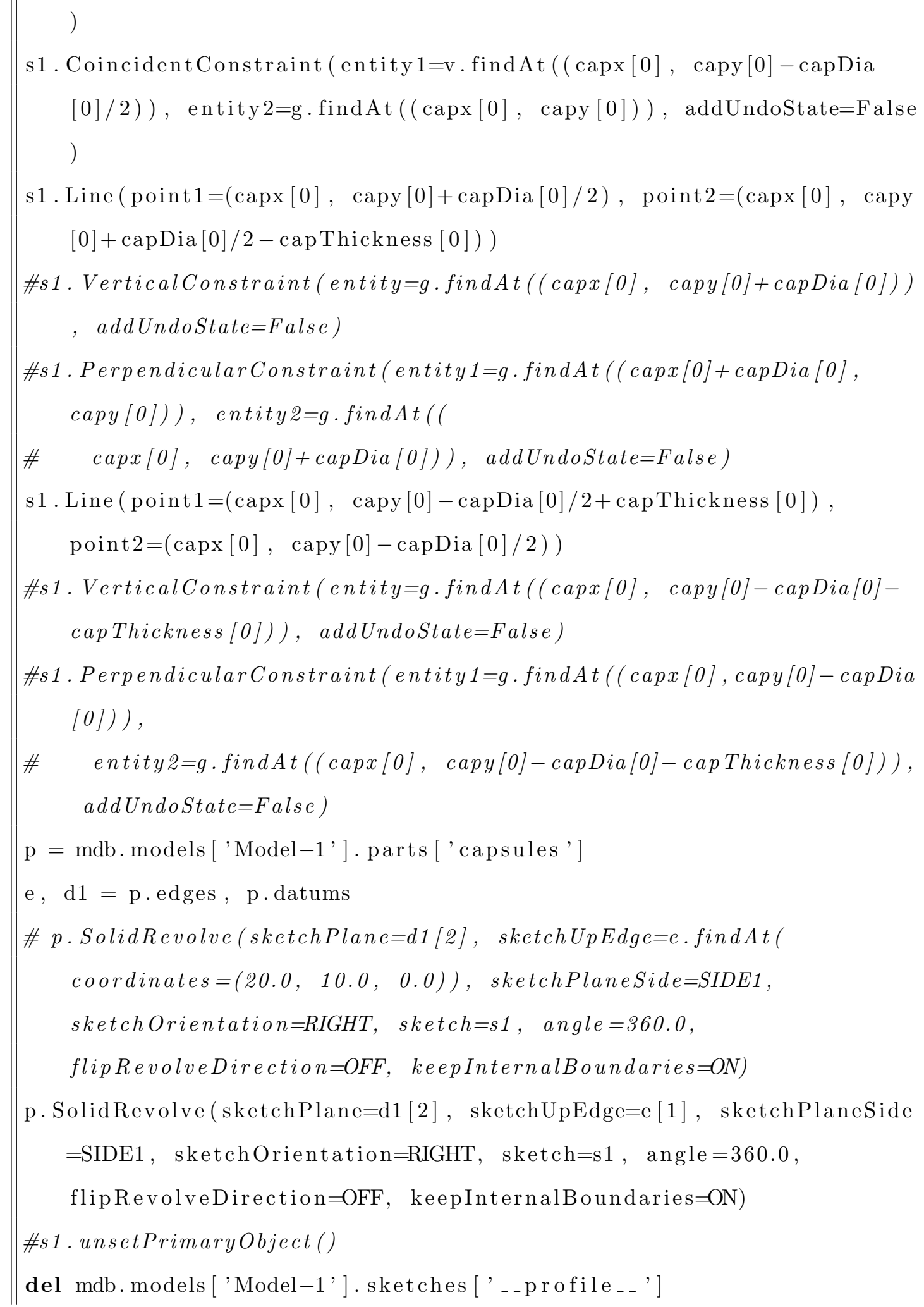


\#2nd capsule

for i in $\operatorname{range}(1, \operatorname{len}(\operatorname{capx}))$ :

$\mathrm{p}=\operatorname{mdb} \cdot \operatorname{models}[$ 'Model-1'].parts ['capsules']

session . viewports ['Viewport: ${ }_{\llcorner} 1$ '] . setValues ( displayedObject=p)

$\mathrm{p}=\operatorname{mdb} \cdot \operatorname{models}[$ 'Model-1'].parts ['capsules']

p. DatumPlaneByPrincipalPlane (principalPlane=XYPLANE, offset=capz [i ] )

$\mathrm{p}=\operatorname{mdb} \cdot \operatorname{models}[$ 'Model-1'].parts ['capsules']

e, $\mathrm{d}=\mathrm{p}$.edges, p.datums

$\mathrm{t}=\mathrm{p}$. MakeSketchTransform $(\operatorname{sketchPlane}=\mathrm{d} 1[2+\mathrm{i} * 3], \quad \operatorname{sketch} \mathrm{UpEdge}=\mathrm{e}[1]$, sketchPlaneSide=SIDE1, sketchOrientation=RIGHT, origin $=(0.0,0.0$, capz [i ]))

$\mathrm{s}=$ mdb. models ['Model-1']. ConstrainedSketch (name=' _-profile - $_{-}$, sheetSize $=400$, gridSpacing $=3.42$, transform=t )

$\mathrm{g}, \mathrm{v}, \mathrm{d} 1, \mathrm{c}=\mathrm{s}$.geometry, s.vertices, s.dimensions, s.constraints

\#s. setPrimaryObject (option $=$ SUPERIMPOSE)

$\mathrm{p}=\operatorname{mdb} \cdot \operatorname{models}[$ 'Model-1'].parts ['capsules']

p.projectReferencesOntoSketch $(\operatorname{sketch}=\mathrm{s}, \quad$ filt e r $=$ COPLANAREDGES $)$

s. ArcByCenterEnds $(\operatorname{center}=(\operatorname{capx}[i], \operatorname{capy}[\mathrm{i}]), \operatorname{point} 1=(\operatorname{capx}[\mathrm{i}], \operatorname{capy}[\mathrm{i}]+$ capDia $[i] / 2-\operatorname{capThickness}[\mathrm{i}]), \operatorname{point} 2=(\operatorname{capx}[\mathrm{i}], \operatorname{capy}[\mathrm{i}]-\operatorname{capDia}[\mathrm{i}$ ]/2+capThickness [i ]),

dire ctio $=$ CLOCKWISE)

s. Line $($ point $1=(\operatorname{capx}[i]$, capy $[i]+\operatorname{capDia}[i] / 2-\operatorname{capThickness}[i])$, point 2 $=(\operatorname{capx}[\mathrm{i}], \operatorname{capy}[\mathrm{i}]-\operatorname{capDia}[\mathrm{i}] / 2+\operatorname{capThickness}[\mathrm{i}]))$

s. VerticalConstraint ( entity $=\mathrm{g}[3+\mathrm{i} * 2], \quad$ addUndoState $=$ False $)$

s. Perpendicular Constraint (entity $1=\mathrm{g}[3+\mathrm{i} * 2-1], \quad$ entity $2=\mathrm{g}[3+\mathrm{i} * 2]$, addUndoState $=$ False )

s. ConstructionLine $(\operatorname{point} 1=(\operatorname{capx}[\mathrm{i}], \operatorname{capy}[\mathrm{i}]+\operatorname{capDia}[\mathrm{i}] / 2-\operatorname{cap}$ Thickness [

i ] $), \operatorname{point} 2=(\operatorname{capx}[\mathrm{i}], \operatorname{capy}[\mathrm{i}]-\operatorname{capDia}[\mathrm{i}] / 2+\operatorname{capThickness}[\mathrm{i}]))$

s. VerticalConstraint ( entity $=\mathrm{g}[4+\mathrm{i} * 2]$, addUndoState $=$ False $)$ 


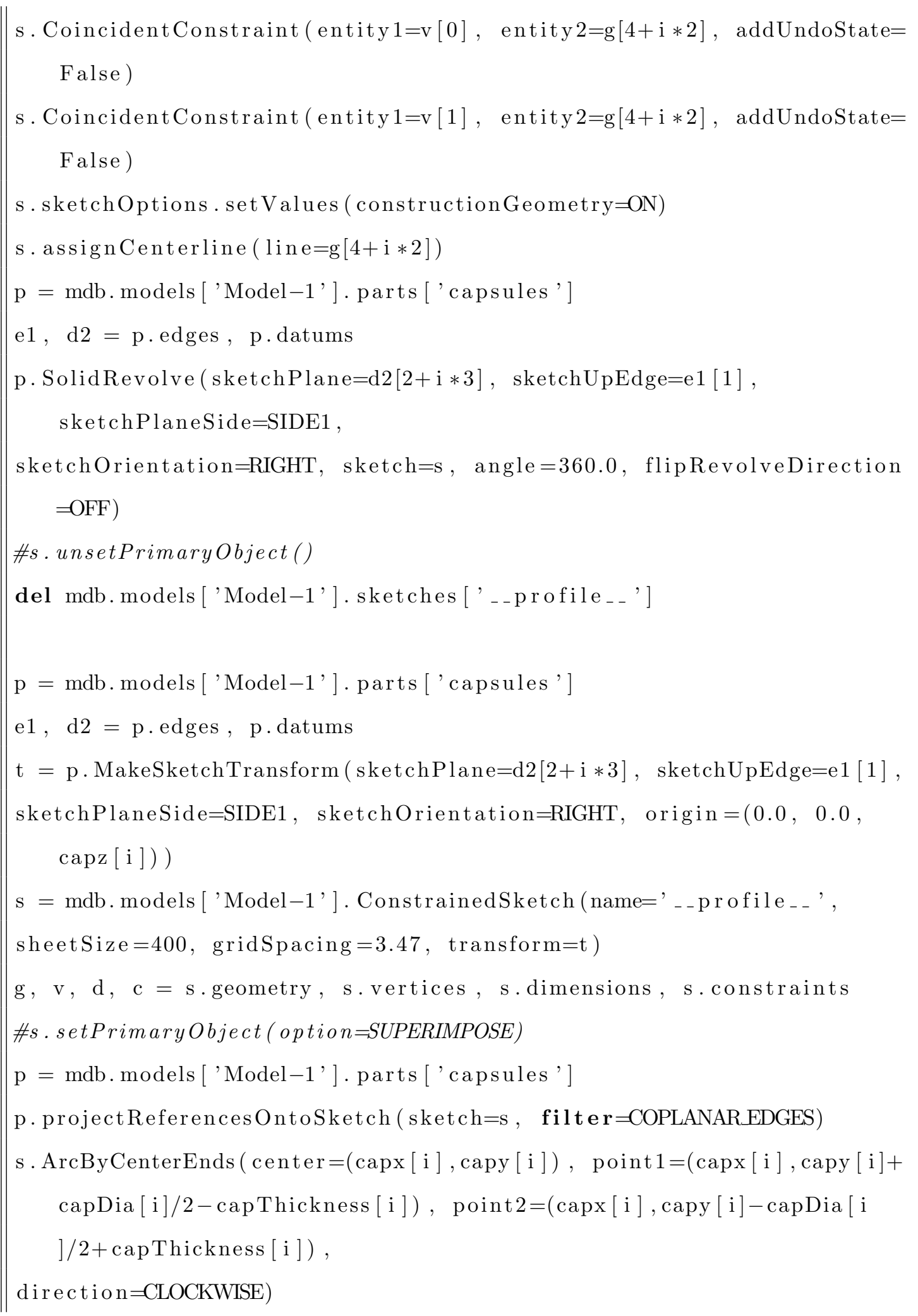




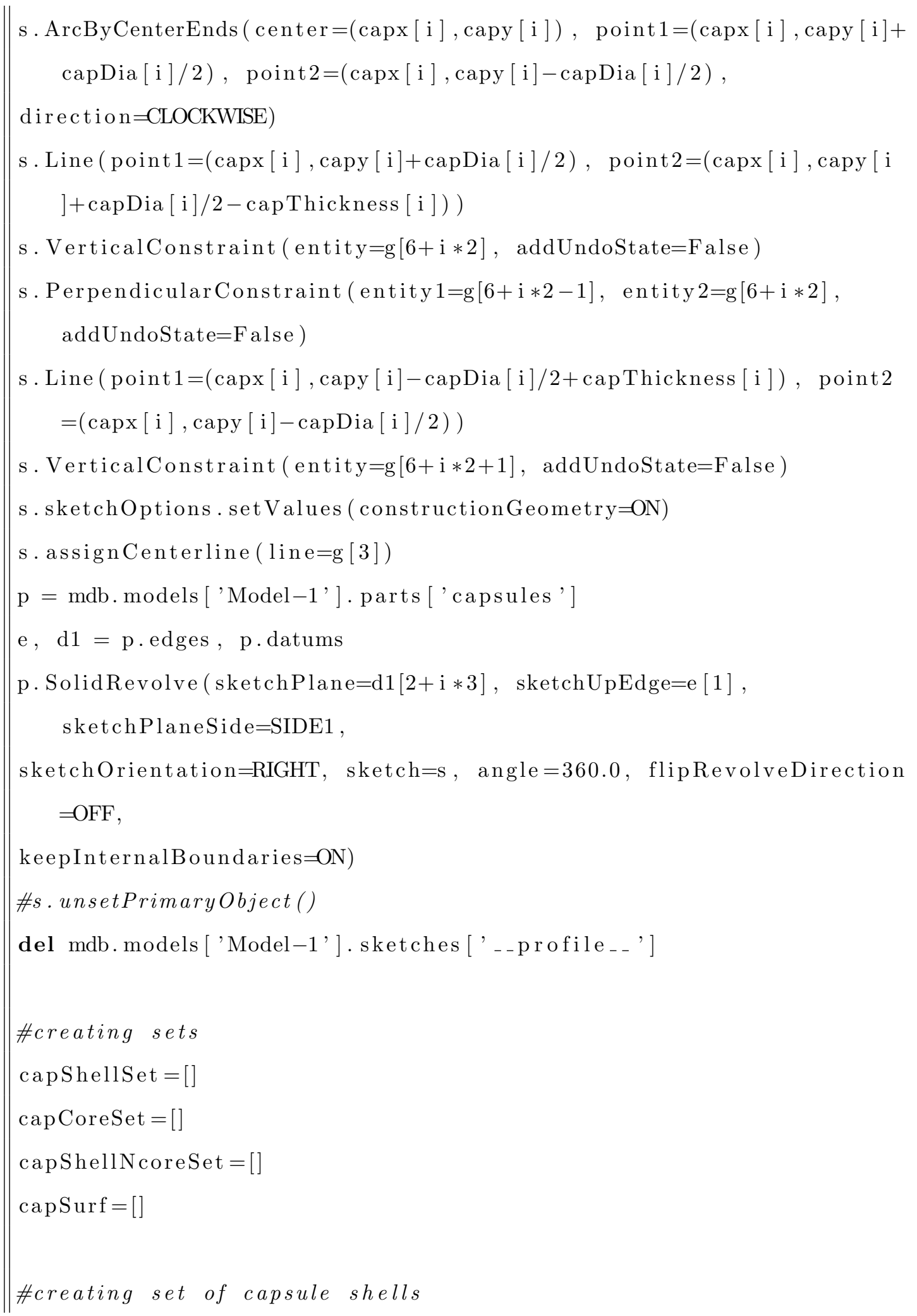




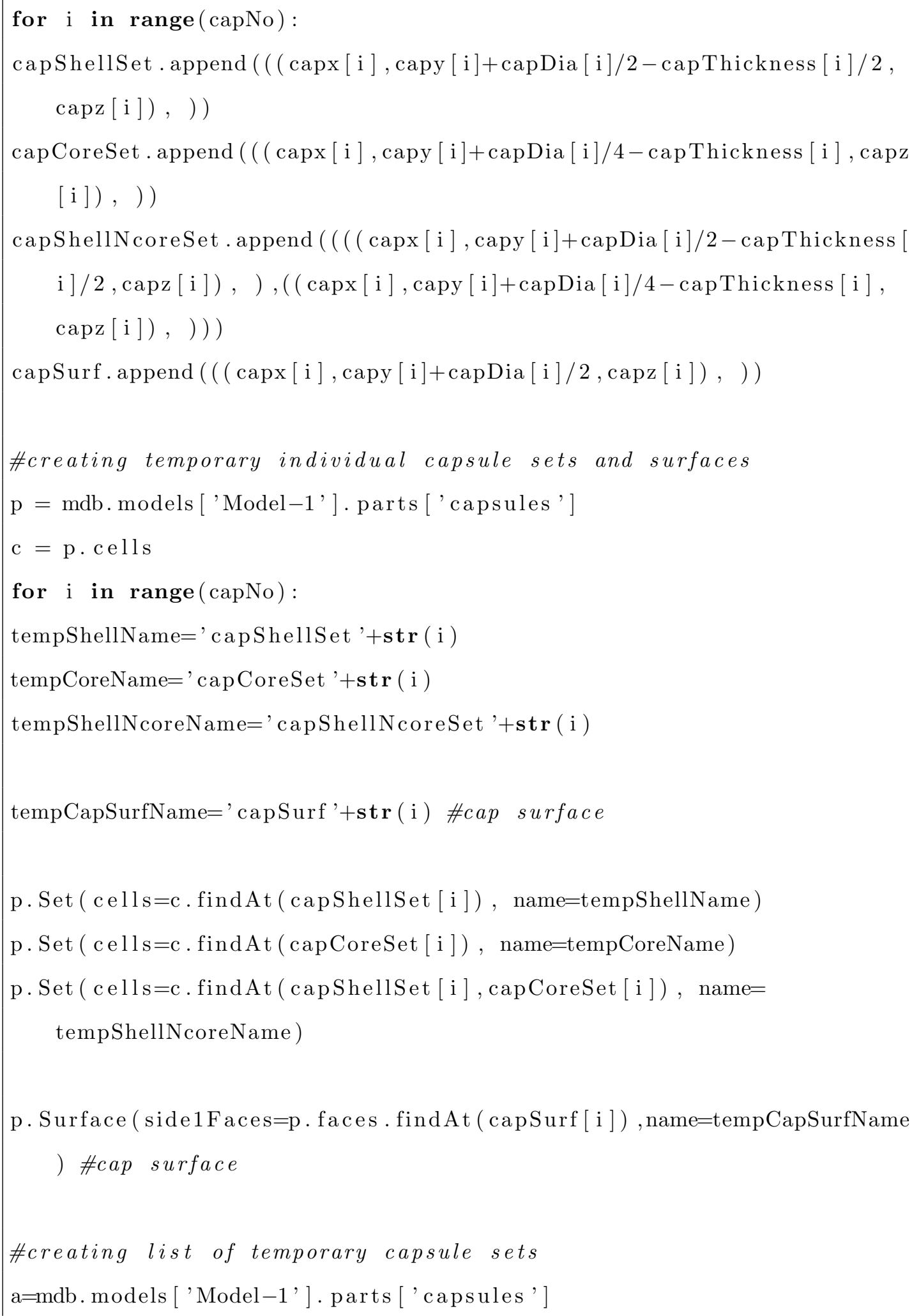




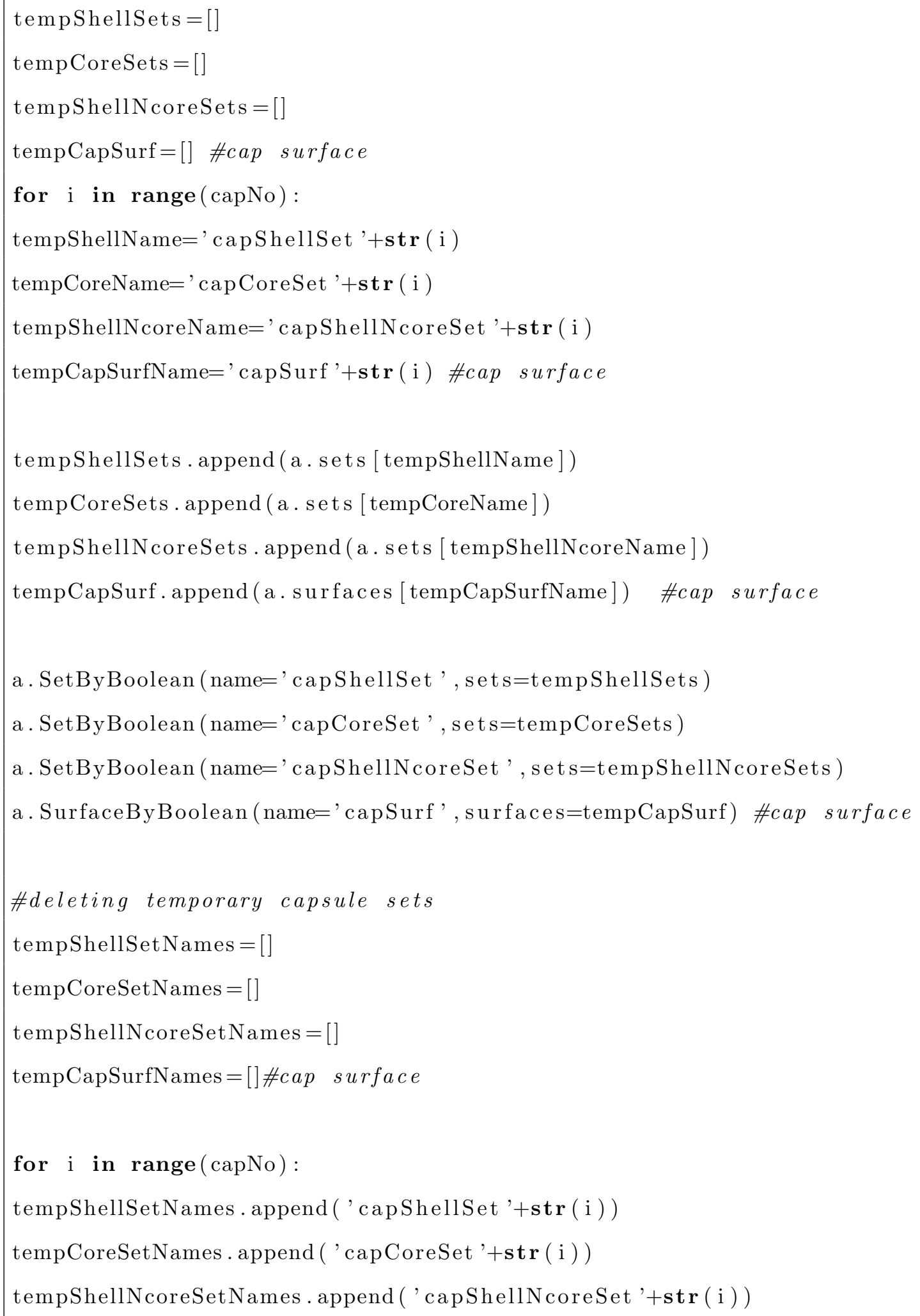




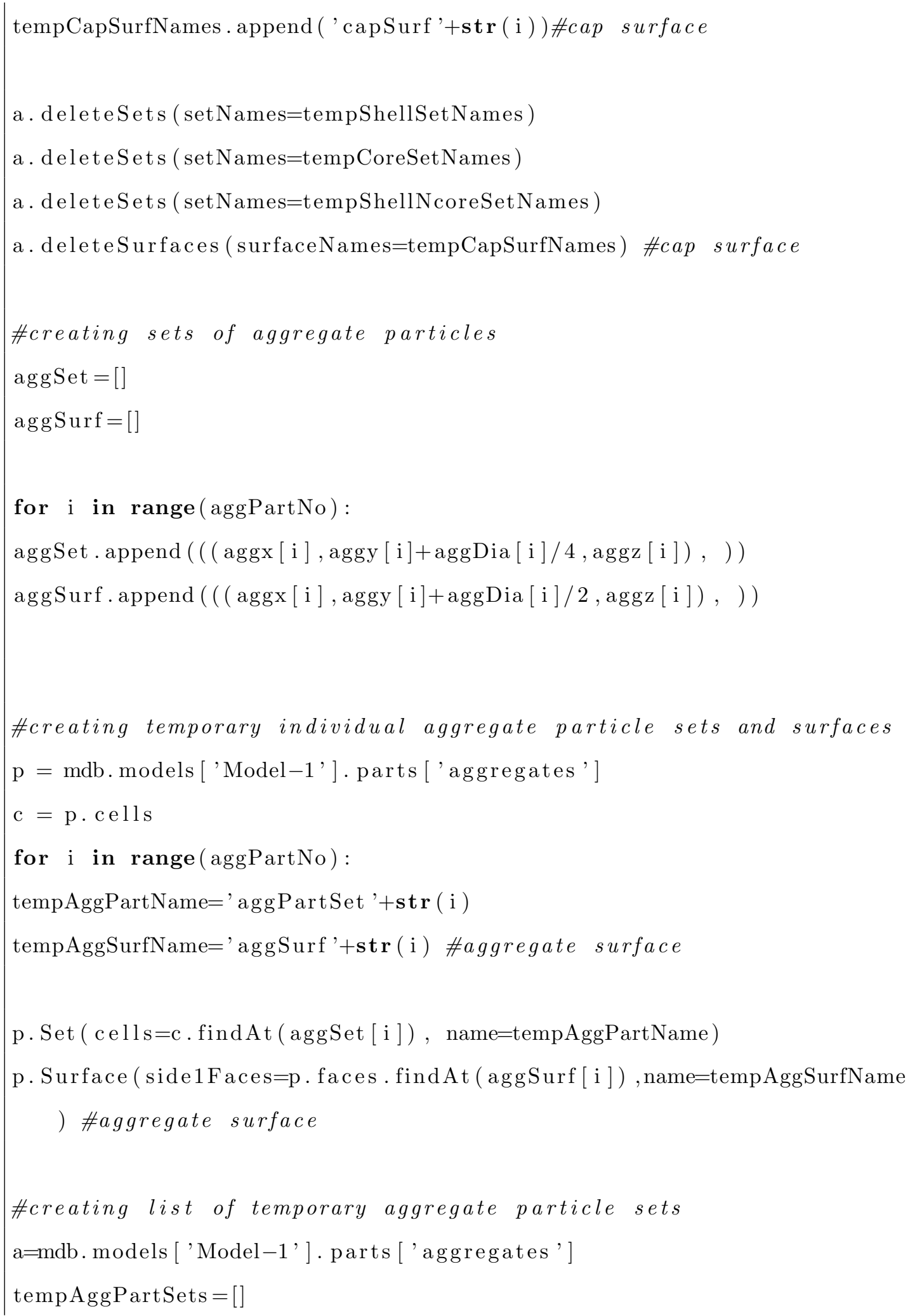




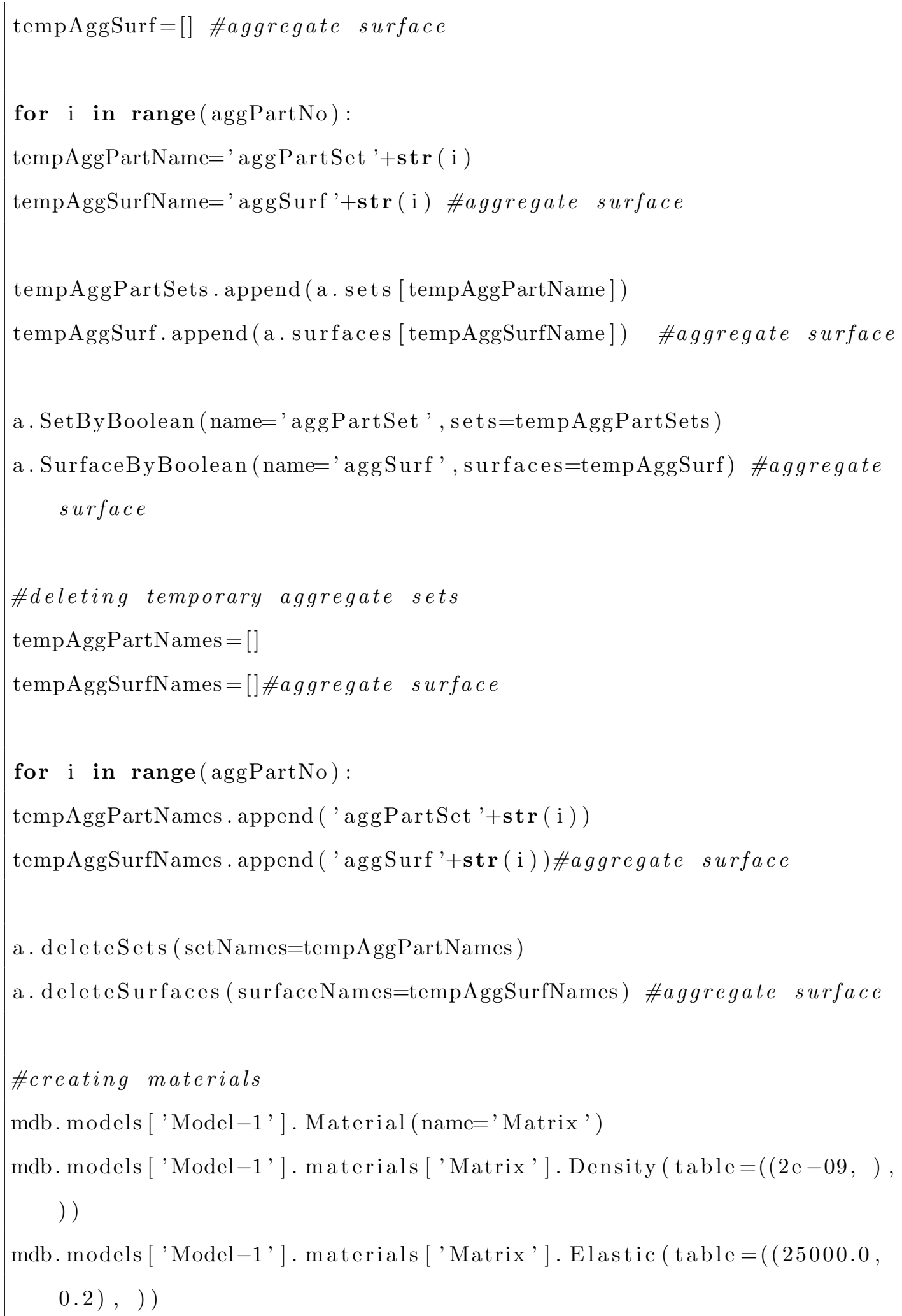




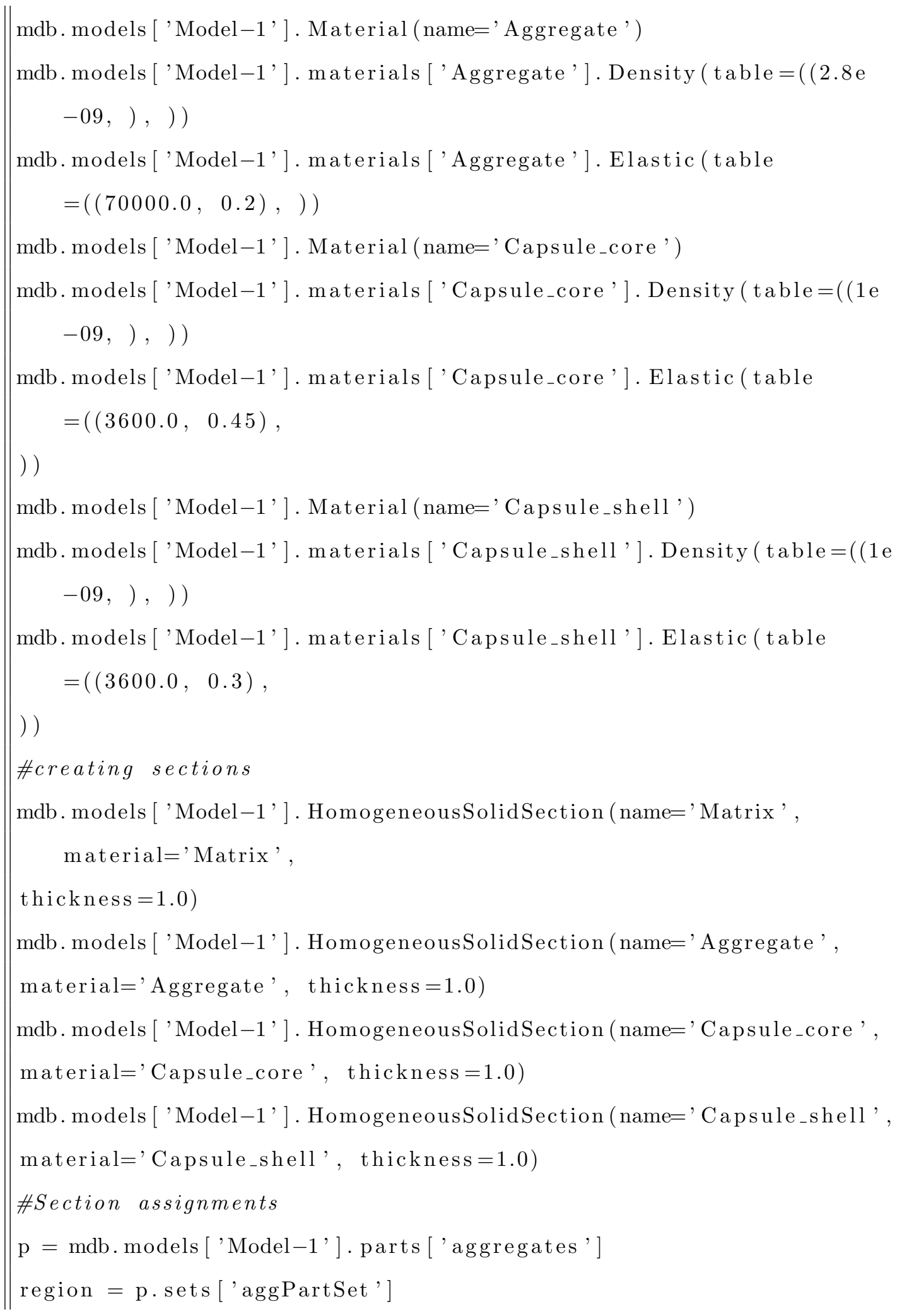




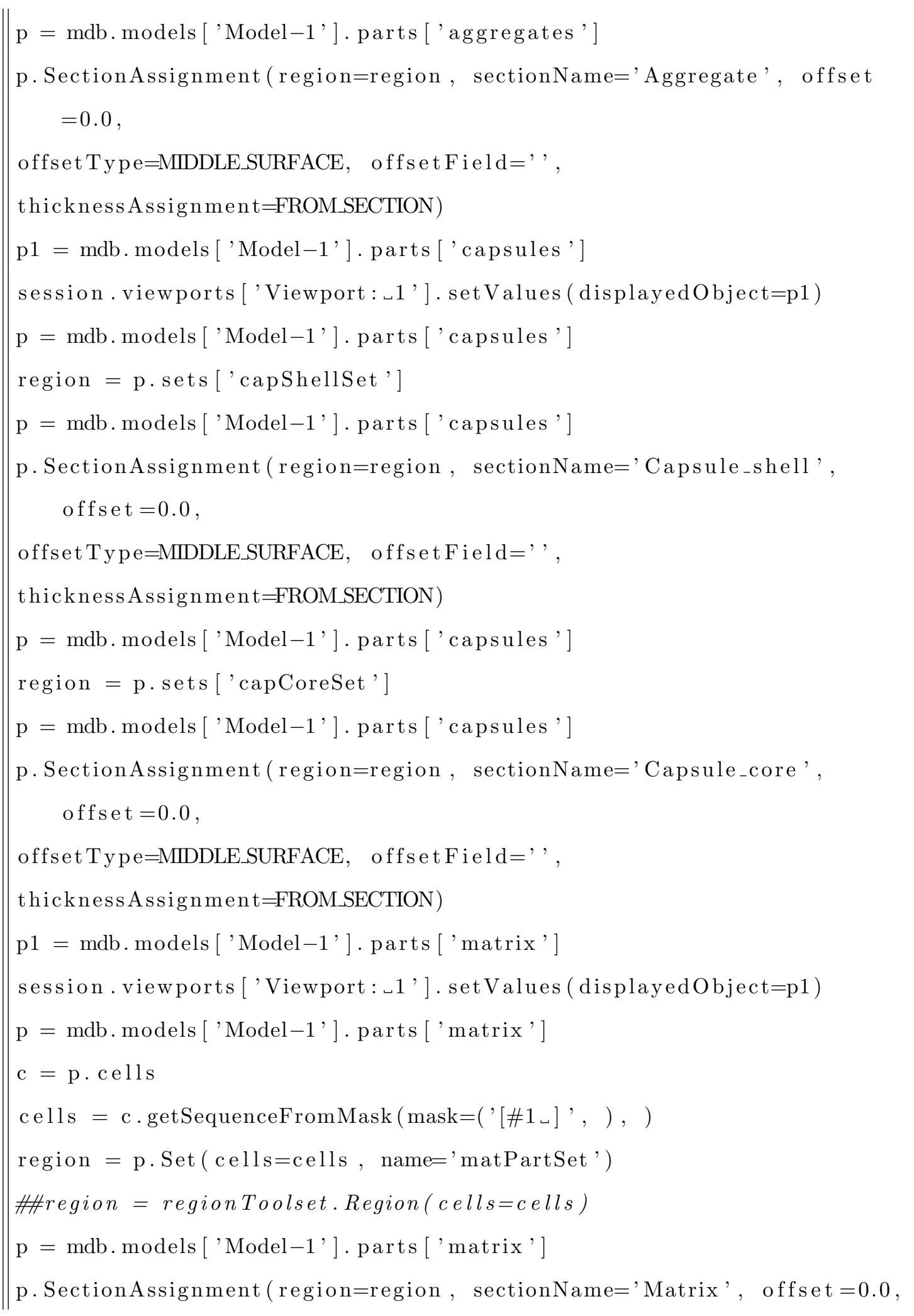




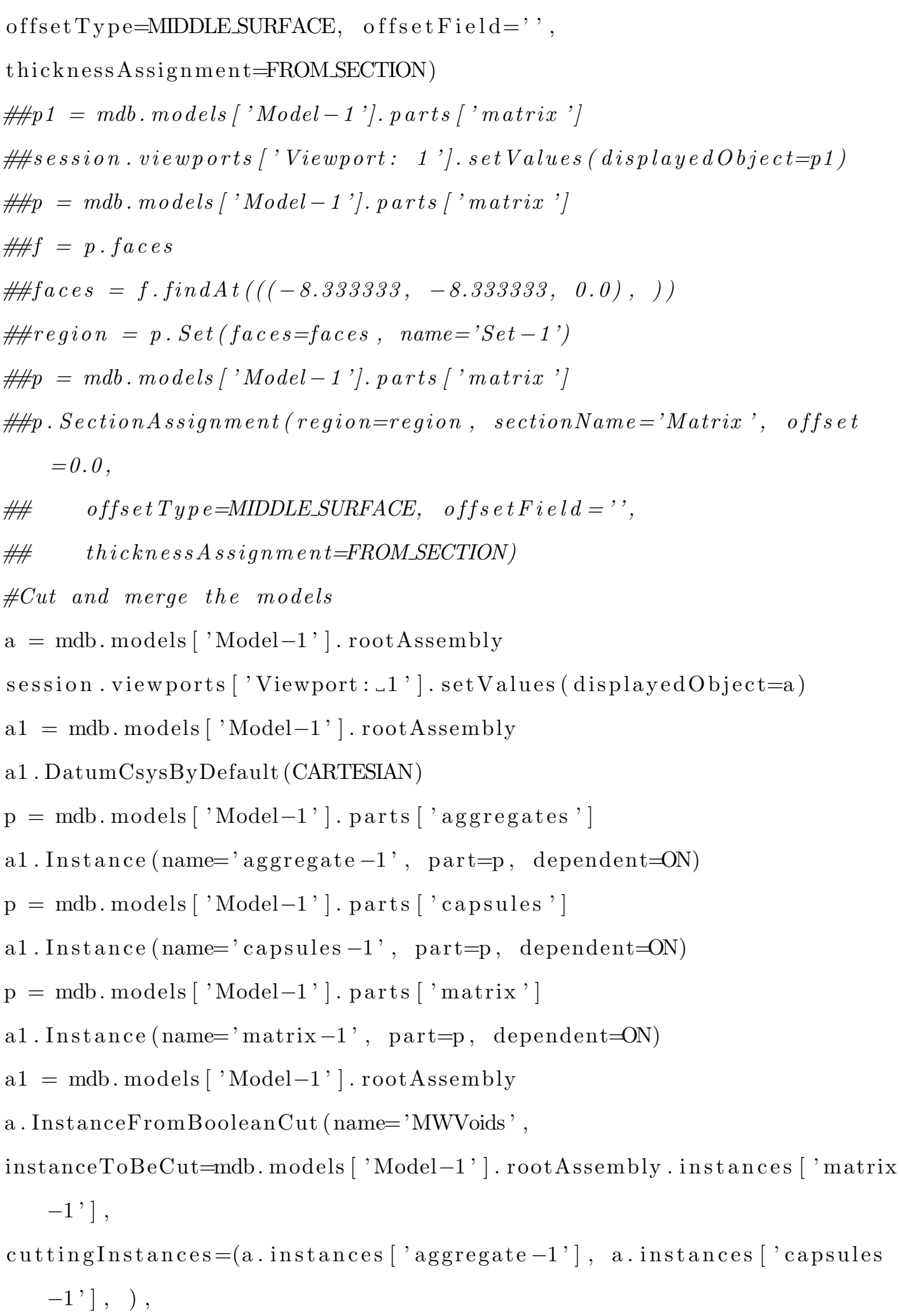




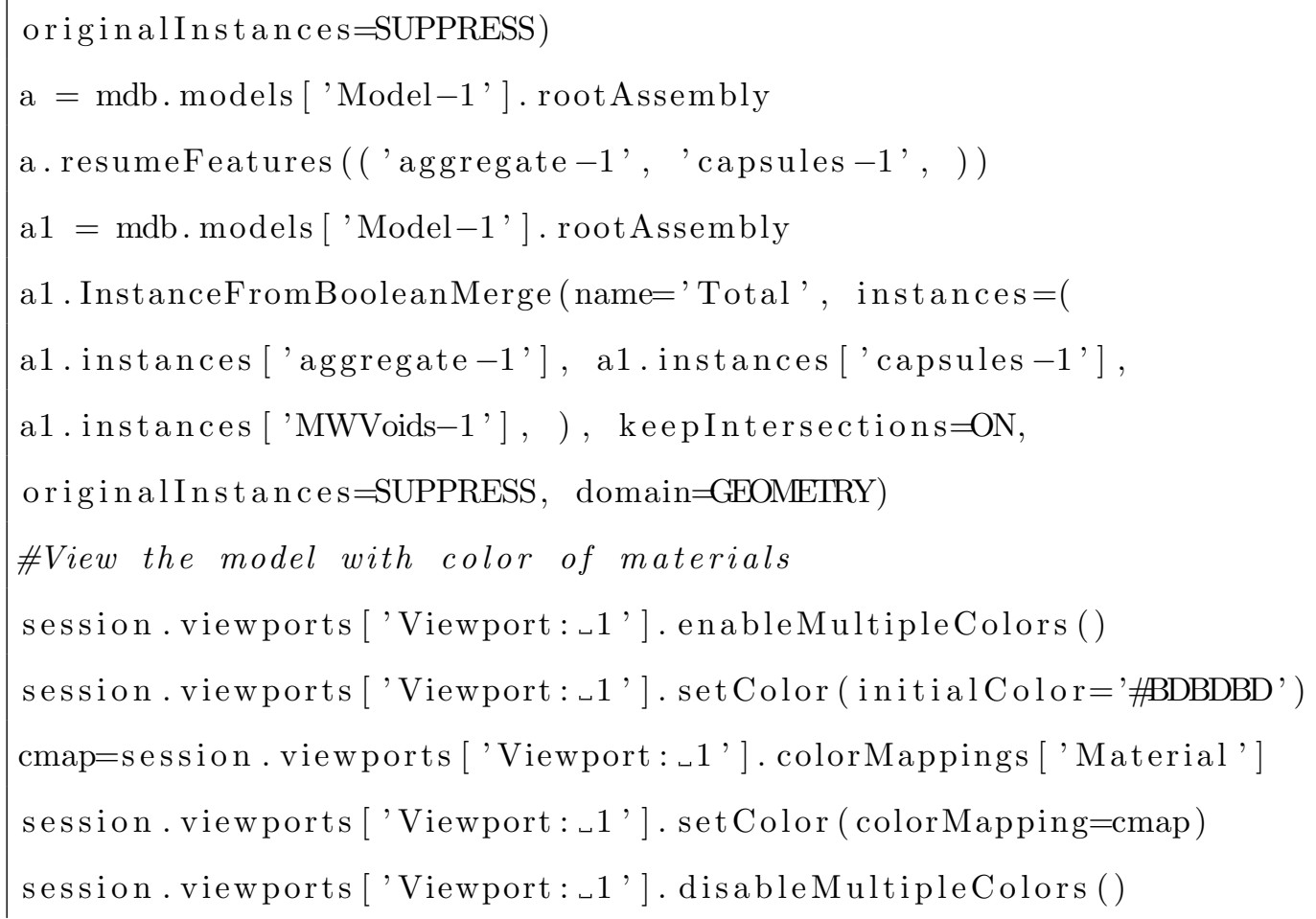




\section{References}

Rashid K Abu Al-Rub and Ammar Alsheghri. Cohesive zone damage-healing model for self-healing materials. In Applied Mechanics and Materials, volume 784, pages 111-118. Trans Tech Publ, 2015. 21

Eunjong Ahn, Hyunjun Kim, Sung-Han Sim, Sung Woo Shin, and Myoungsu Shin. Principles and applications of ultrasonic-based nondestructive methods for self-healing in cementitious materials. Materials, 10(3):278, 2017. 17

Tae-Ho Ahn and Toshiharu Kishi. Crack self-healing behavior of cementitious composites incorporating various mineral admixtures. Journal of Advanced Concrete Technology, 8(2):171-186, 2010. 18

Alexander Alexeev, Rolf Verberg, and Anna C Balazs. Patterned surfaces segregate compliant microcapsules. Langmuir, 23(3):983-987, 2007. 36

A Aliko-Benítez, M Doblaré, and JA Sanz-Herrera. Chemical-diffusive modeling of the self-healing behavior in concrete. International Journal of Solids and Structures, 69:392-402, 2015. 21

Fatemeh Amiri, C Anitescu, M Arroyo, Stephane Pierre Alain Bordas, and Timon Rabczuk. Xlme interpolants, a seamless bridge between xfem and enriched meshless methods. Computational Mechanics, 53(1):45-57, 2014. 7

P Areias, T Rabczuk, and D Dias-da Costa. Element-wise fracture algorithm based on rotation of edges. Engineering Fracture Mechanics, 110:113-137, 2013. 7 
P Areias, MA Msekh, and T Rabczuk. Damage and fracture algorithm using the screened poisson equation and local remeshing. Engineering Fracture Mechanics, 158:116-143, 2016a. 7

P Areias, T Rabczuk, and MA Msekh. Phase-field analysis of finite-strain plates and shells including element subdivision. Computer Methods in Applied Mechanics and Engineering, 312:322-350, 2016b. 7

Pedro Areias, Timon Rabczuk, and PP Camanho. Finite strain fracture of $2 \mathrm{~d}$ problems with injected anisotropic softening elements. Theoretical and Applied Fracture Mechanics, 72:50-63, 2014. 7

Ever J Barbero, Fabrizio Greco, and Paolo Lonetti. Continuum damage-healing mechanics with application to self-healing composites. International Journal of Damage Mechanics, 14(1):51-81, 2005. 2

Zdeněk P Bažant and Pietro G Gambarova. Crack shear in concrete: Crack band microflane model. Journal of Structural Engineering, 110(9):2015-2035, 1984. 10

Zdenek P Bazant and Byung H Oh. Microplane model for fracture analysis of concrete structures. Technical report, NORTHWESTERN UNIV EVANSTON IL TECHNOLOGICAL INST, 1983. 10

Zdeněk P Bažant, Yuyin Xiang, and Pere C Prat. Microplane model for concrete. i: Stress-strain boundaries and finite strain. Journal of Engineering Mechanics, $122(3): 245-254,1996.10$

T. Belytschko, Y. Y. Lu, and L. Gu. Element-free galerkin methods. International Journal for Numerical Methods in Engineering, 37(2):229-256, 1994a. ISSN 1097-0207. doi: 10.1002/nme.1620370205. URL http://dx.doi.org/ 10.1002/nme.1620370205. 43

Ted Belytschko and Jeong-Hoon Song. Coarse-graining of multiscale crack propagation. International journal for numerical methods in engineering, 81(5): 537-563, 2010. 7 
Ted Belytschko, Yun Yun Lu, and Lei Gu. Element-free galerkin methods. International journal for numerical methods in engineering, 37(2):229-256, 1994b. 8

M Bruggi, S Casciati, and L Faravelli. Cohesive crack propagation in a random elastic medium. Probabilistic Engineering Mechanics, 23(1):23-35, 2008. 14

Pattabhi R Budarapu, Robert Gracie, Stéphane PA Bordas, and Timon Rabczuk. An adaptive multiscale method for quasi-static crack growth. Computational Mechanics, 53(6):1129-1148, 2014a. 7

Pattabhi R Budarapu, Robert Gracie, Shih-Wei Yang, Xiaoying Zhuang, and Timon Rabczuk. Efficient coarse graining in multiscale modeling of fracture. Theoretical and Applied Fracture Mechanics, 69:126-143, 2014b. 7

Antonio Caggiano, Guillermo Etse, Liberato Ferrara, and Visar Krelani. Zerothickness interface constitutive theory for concrete self-healing effects. Computers \& Structures, 186:22-34, 2017. 22

E Cailleux and V Pollet. Investigations on the development of self-healing properties in protective coatings for concrete and repair mortars. In Proceedings of the 2nd International Conference on Self-Healing Materials, Chicago, IL, USA, volume 28, 2009. 1

Ignacio Carol, Egidio Rizzi, and Kaspar Willam. On the formulation of anisotropic elastic degradation. i. theory based on a pseudo-logarithmic damage tensor rate. International Journal of Solids and Structures, 38(4):491-518, 2001. 9

Jan Červenka and Vassilis K Papanikolaou. Three dimensional combined fracture-plastic material model for concrete. International journal of plasticity, 24(12):2192-2220, 2008. 9

Adriana Silviana Chitez and Anthony Duncan Jefferson. A coupled thermo-hygrochemical model for characterising autogenous healing in ordinary cementitious materials. Cement and Concrete Research, 88:184-197, 2016. 22 
Masoud K Darabi, Rashid K Abu Al-Rub, and Dallas N Little. A continuum damage mechanics framework for modeling micro-damage healing. International Journal of Solids and Structures, 49(3-4):492-513, 2012. 2

M Laurent Daudeville. Role of coarse aggregates in the triaxial behavior of concrete: experimental and numerical analysis. PhD thesis, Citeseer, 2014. 37, 81

Robert Davies and Anthony Jefferson. Micromechanical modelling of self-healing cementitious materials. International Journal of Solids and Structures, 113: 180-191, 2017. ix, 22, 23

G Di Luzio, L Ferrara, and V Krelani. A numerical model for the self-healing capacity of cementitious composites. In Proceedings of EURO-C, volume 2, pages $741-7,2014.19$

Jelena M Djoković, Ružica R Nikolić, and Jan Bujnak. Fundamental problems of modeling the fracture processes in concrete i: Micromechanics and localization of damages. Procedia Engineering, 65:186-195, 2013a. 8

Jelena M Djoković, Ružica R Nikolić, and Jan Bujnak. Fundamental problems of modeling the fracture processes in concrete ii: Size effect and selection of the solution approach. Procedia Engineering, 65:196-205, 2013b. 8

Biqin Dong, Yanshuai Wang, Guohao Fang, Ningxu Han, Feng Xing, and Youyuan Lu. Smart releasing behavior of a chemical self-healing microcapsule in the stimulated concrete pore solution. Cement and Concrete Composites, $56: 46-50,2015.65$

Xiuli Du, Liu Jin, and Guowei Ma. Numerical modeling tensile failure behavior of concrete at mesoscale using extended finite element method. International Journal of Damage Mechanics, 23(7):872-898, 2014. ix, 12, 14, 37, 81

Carola Edvardsen. Water permeability and autogenous healing of cracks in concrete. Materials Journal, 96(4):448-454, 1999. 18 
Liberato Ferrara, Giovanni DI LUZIO, Visar Krelani, et al. Experimental assessment and numerical modeling of self healing capacity of cement based materials via fracture mechanics concepts. In FraMCoS 9, pages 1-12, 2016. 19

Paula Folino and Guillermo Etse. Performance dependent model for normal and high strength concretes. International Journal of Solids and Structures, 49(5): 701-719, 2012. 9

Shao-Yun Fu, Xi-Qiao Feng, Bernd Lauke, and Yiu-Wing Mai. Effects of particle size, particle/matrix interface adhesion and particle loading on mechanical properties of particulate-polymer composites. Composites Part B: Engineering, 39(6):933-961, 2008. 65

H. Ghasemi, H.S. Park, and T. Rabczuk. A level-set based iga formulation for topology optimization of flexoelectric materials. Computer Methods in Applied Mechanics and Engineering, 313:239-258, 2017. 43

S.S. Ghorashi, N. Valizadeh, S. Mohammadi, and T. Rabczuk. T-spline based xiga for fracture analysis of orthotropic media. Computers and Structures, 147: 138-146, 2015. 43

FA Gilabert, D Garoz, and Wim Van Paepegem. Stress concentrations and bonding strength in encapsulation-based self-healing materials. Materials $\&$ Design, 67:28-41, 2015. x, 2, 29, 30, 46, 80, 86, 88

Lori Graham-Brady and X Frank Xu. Stochastic morphological modeling of random multiphase materials. Journal of Applied Mechanics, 75(6):061001, 2008. 14

Peter Grassl. On a damage-plasticity approach to model concrete failure. arXiv preprint arXiv:0811.2776, 2008. 10

Peter Grassl and Milan Jirásek. Damage-plastic model for concrete failure. International journal of solids and structures, 43(22):7166-7196, 2006. 10

Peter Grassl and Milan Jirásek. Meso-scale approach to modelling the fracture process zone of concrete subjected to uniaxial tension. International Journal of Solids and Structures, 47(7):957-968, 2010. 12 
Peter Grassl, Karin Lundgren, and Kent Gylltoft. Concrete in compression: a plasticity theory with a novel hardening law. International Journal of Solids and Structures, 39(20):5205-5223, 2002. 9

Peter Grassl, David Grégoire, Laura Rojas Solano, and Gilles Pijaudier-Cabot. Meso-scale modelling of the size effect on the fracture process zone of concrete. International Journal of Solids and Structures, 49(13):1818-1827, 2012. 15

Elke Gruyaert, Kim Van Tittelboom, Jelle Sucaet, Johannes Anrijs, Sandra Van Vlierberghe, Peter Dubruel, BG De Geest, Jean-Paul Remon, and Nele De Belie. Capsules with evolving brittleness to resist the preparation of selfhealing concrete. Materiales de Construcción, 66(323):092, 2016. 37, 82

Tobias Hazelwood, Anthony Duncan Jefferson, Robert John Lark, and Diane Ruth Gardner. Numerical simulation of the long-term behaviour of a selfhealing concrete beam vs standard reinforced concrete. Engineering Structures, 102:176-188, 2015. 21

Huan He, Zhanqi Guo, Piet Stroeven, Martijn Stroeven, and Lambertus Johannes Sluys. Self-healing capacity of concrete-computer simulation study of unhydrated cement structure. Image Analysis \& Stereology, 26(3):137-143, 2011. 18

Nataliya Hearn. Self-sealing, autogenous healing and continued hydration: What is the difference? Materials and Structures, 31(8):563-567, 1998. 18

Arne Hillerborg, Mats Modéer, and P-E Petersson. Analysis of crack formation and crack growth in concrete by means of fracture mechanics and finite elements. Cement and concrete research, 6(6):773-781, 1976. 7, 8

Benoît Hilloulin, Frederic Grondin, Mohammed Matallah, and Ahmed Loukili. Modelling of autogenous healing in ultra high performance concrete. Cement and Concrete Research, 61:64-70, 2014. 19

Benoit Hilloulin, Kim Van Tittelboom, Elke Gruyaert, Nele De Belie, and Ahmed Loukili. Design of polymeric capsules for self-healing concrete. Cement and Concrete Composites, 55:298-307, 2015. 3, 36, 37, 46, 66, 80, 82, 86, 88 
Benoit Hilloulin, Damien Hilloulin, Frédéric Grondin, Ahmed Loukili, and Nele De Belie. Mechanical regains due to self-healing in cementitious materials: Experimental measurements and micro-mechanical model. Cement and Concrete Research, 80:21-32, 2016. 20, 22

Teddy J Hirsch. Modulus of elasticity of concrete affected by elastic moduli of cement paste matrix and aggregate. In Journal Proceedings, volume 59, pages 427-452, 1962. 36, 37, 81

B Valentini Hofstetter et al. Review and enhancement of 3d concrete models for large-scale numerical simulations of concrete structures. International Journal for Numerical and Analytical Methods in Geomechanics, 37(3):221-246, 2013. 10

Haoliang Huang and Guang Ye. Simulation of self-healing by further hydration in cementitious materials. Cement and Concrete Composites, 34(4):460-467, 2012. ix, 18, 19, 20

Haoliang Huang and Guang Ye. Numerical studies of the effects of water capsules on self-healing efficiency and mechanical properties in cementitious materials. Advances in Materials Science and Engineering, 2016, 2016. x, 31

Thomas JR Hughes, John A Cottrell, and Yuri Bazilevs. Isogeometric analysis: Cad, finite elements, nurbs, exact geometry and mesh refinement. Computer methods in applied mechanics and engineering, 194(39):4135-4195, 2005. 43

Ludovic Jason, Antonio Huerta, Gilles Pijaudier-Cabot, and Shahrokh Ghavamian. An elastic plastic damage formulation for concrete: Application to elementary tests and comparison with an isotropic damage model. Computer methods in applied mechanics and engineering, 195(52):7077-7092, 2006. 10

L Jendele, J Cervenka, V Saouma, and R Pukl. On the choice between discrete or smeared approach in practical structural fe analyses of concrete structures. In Fourth International Conference on Analysis of Discontinuous Deformation Glasgow, Scotland UK, 2001. 8 
Milan Jirásek and Thomas Zimmermann. Rotating crack model with transition to scalar damage. Journal of engineering mechanics, 124(3):277-284, 1998. 8

Antonis Kanellopoulos, Petros Giannaros, and Abir Al-Tabbaa. The effect of varying volume fraction of microcapsules on fresh, mechanical and self-healing properties of mortars. Construction and Building Materials, 122:577-593, 2016. 55,100

MW Keller and NR Sottos. Mechanical properties of microcapsules used in a self-healing polymer. Experimental Mechanics, 46(6):725-733, 2006. 46, 69, 87, 88

Phaedon S Koutsourelakis and George Deodatis. Simulation of multidimensional binary random fields with application to modeling of two-phase random media. Journal of engineering mechanics, 132(6):619-631, 2006. 14

Wenting Li, Zhengwu Jiang, and Zhenghong Yang. Crack extension and possibility of debonding in encapsulation-based self-healing materials. Materials, 10 (6):589, 2017. 34

Carlos M López, Ignacio Carol, and Antonio Aguado. Meso-structural study of concrete fracture using interface elements. i: numerical model and tensile behavior. Materials and structures, 41(3):583-599, 2008a. 12

Carlos M López, Ignacio Carol, and Antonio Aguado. Meso-structural study of concrete fracture using interface elements. ii: compression, biaxial and brazilian test. Materials and Structures, 41(3):601-620, 2008b. 12

Leyang Lv, Erik Schlangen, Zhengxian Yang, and Feng Xing. Micromechanical properties of a new polymeric microcapsule for self-healing cementitious materials. Materials, 9(12):1025, 2016. 55, 100

Z Lv and D Chen. Overview of recent work on self-healing in cementitious materials. Materiales de Construcción, 64(316):034, 2014. 17

Zhong Lv and Huisu Chen. Modeling of self-healing efficiency for cracks due to unhydrated cement nuclei in hardened cement paste. Procedia Engineering, 27: 281-290, 2012. 18 
Zhong Lv and Huisu Chen. Analytical models for determining the dosage of capsules embedded in self-healing materials. Computational Materials Science, 68:81-89, 2013. x, 2, 26, 27, 80

Zhong Lv, Huisu Chen, and Haifeng Yuan. Analytical solution on dosage of self-healing agents in cementitious materials: long capsule model. Journal of Intelligent Material Systems and Structures, 25(1):47-57, 2014. x, 2, 28, 29, 80

Mathias Maes, Kim Van Tittelboom, and Nele De Belie. The efficiency of selfhealing cementitious materials by means of encapsulated polyurethane in chloride containing environments. Construction and Building Materials, 71:528537, 2014. 65

Matbase. Pmma. URL https://www.matbase.com/material-categories/ natural-and-synthetic-polymers/thermoplastics/ commodity-polymers/material-properties-of-polymethyl-methacrylate-extruded-acr html\#properties. 45, 87

Philippe Menetrey and KJ Willam. Triaxial failure criterion for concrete and its generalization. Structural Journal, 92(3):311-318, 1995. 9

Nicolas Moës and Ted Belytschko. Extended finite element method for cohesive crack growth. Engineering fracture mechanics, 69(7):813-833, 2002. 7

Thomas Most. Stochastic crack growth simulation in reinforced concrete structures by means of coupled finite element and meshless methods. 2005. 14

Giang D Nguyen and Guy T Houlsby. A coupled damage-plasticity model for concrete based on thermodynamic principles: Part i: model formulation and parameter identification. International journal for numerical and analytical methods in geomechanics, 32(4):353-389, 2008. 10

Giang D Nguyen and Alexander M Korsunsky. Development of an approach to constitutive modelling of concrete: isotropic damage coupled with plasticity. International Journal of Solids and Structures, 45(20):5483-5501, 2008. 10 
V.P. Nguyen, T. Rabczuk, S. Bordas, and M. Duflot. Meshless methods: A review and computer implementation aspects. Mathematics and Computers in Simulation, 79(3):763-813, 2008. 43

V.P. Nguyen, C. Anitescu, S.P.A. Bordas, and T. Rabczuk. Isogeometric analysis: An overview and computer implementation aspects. Mathematics and Computers in Simulation, 117:89-116, 2015. 43

N. Nguyen-Thanh, J. Kiendl, H. Nguyen-Xuan, R. Wchner, K.U. Bletzinger, Y. Bazilevs, and T. Rabczuk. Rotation free isogeometric thin shell analysis using pht-splines. Computer Methods in Applied Mechanics and Engineering, 200(47-48):3410-3424, 2011a. 43

N. Nguyen-Thanh, H. Nguyen-Xuan, S.P.A. Bordas, and T. Rabczuk. Isogeometric analysis using polynomial splines over hierarchical t-meshes for twodimensional elastic solids. Computer Methods in Applied Mechanics and Engineering, 200(21-22):1892-1908, 2011b. 43

N. Nguyen-Thanh, N. Valizadeh, M.N. Nguyen, H. Nguyen-Xuan, X. Zhuang, P. Areias, G. Zi, Y. Bazilevs, L. De Lorenzis, and T. Rabczuk. An extended isogeometric thin shell analysis based on kirchhoff-love theory. Computer Methods in Applied Mechanics and Engineering, 284:265-291, 2015. 43

J Oliver, Alfredo Edmundo Huespe, E Samaniego, and EWV Chaves. Continuum approach to the numerical simulation of material failure in concrete. International journal for numerical and analytical methods in geomechanics, 28(7-8): 609-632, 2004. 10

Michael Ortiz. A constitutive theory for the inelastic behavior of concrete. Mechanics of materials, 4(1):67-93, 1985. 9

Joško Ožbolt, Yijun Li, and Ivica Kožar. Microplane model for concrete with relaxed kinematic constraint. International Journal of Solids and Structures, 38(16):2683-2711, 2001. ix, 10, 11 
Vassilis K Papanikolaou and Andreas J Kappos. Confinement-sensitive plasticity constitutive model for concrete in triaxial compression. International Journal of Solids and Structures, 44(21):7021-7048, 2007. 9

Md Shahriar Quayum, Xiaoying Zhuang, and Timon Rabczuk. Computational model generation and rve design of self-healing concrete. Frontiers of Structural and Civil Engineering, 9(4):383-396, 2015. 38

T Rabczuk and T Belytschko. Cracking particles: a simplified meshfree method for arbitrary evolving cracks. International Journal for Numerical Methods in Engineering, 61(13):2316-2343, 2004. 7, 8

T Rabczuk and Ted Belytschko. Application of particle methods to static fracture of reinforced concrete structures. International Journal of Fracture, 137(1-4): 19-49, 2006. ix, 8, 9

T. Rabczuk and E. Samaniego. Discontinuous modelling of shear bands using adaptive meshfree methods. Computer Methods in Applied Mechanics and Engineering, 197(6-8):641-658, 2008. 42

T Rabczuk, J Akkermann, and J Eibl. A numerical model for reinforced concrete structures. International Journal of Solids and Structures, 42(5):1327-1354, 2005. 8

T. Rabczuk, P.M.A. Areias, and T. Belytschko. A meshfree thin shell method for non-linear dynamic fracture. International Journal for Numerical Methods in Engineering, 72(5):524-548, 2007a. 42

T. Rabczuk, S. Bordas, and G. Zi. A three-dimensional meshfree method for continuous multiple-crack initiation, propagation and junction in statics and dynamics. Computational Mechanics, 40(3):473-495, 2007b. 42

T. Rabczuk, G. Zi, S. Bordas, and H. Nguyen-Xuan. A geometrically non-linear three-dimensional cohesive crack method for reinforced concrete structures. Engineering Fracture Mechanics, 75(16):4740-4758, 2008a. 42 
T. Rabczuk, G. Zi, A. Gerstenberger, and W.A. Wall. A new crack tip element for the phantom-node method with arbitrary cohesive cracks. International Journal for Numerical Methods in Engineering, 75(5):577-599, 2008b. 42

T. Rabczuk, J.-H. Song, and T. Belytschko. Simulations of instability in dynamic fracture by the cracking particles method. Engineering Fracture Mechanics, 76 (6):730-741, 2009. 42

T. Rabczuk, S. Bordas, and G. Zi. On three-dimensional modelling of crack growth using partition of unity methods. Computers and Structures, 88(2324):1391-1411, 2010a. 42

T. Rabczuk, R. Gracie, J.-H. Song, and T. Belytschko. Immersed particle method for fluid-structure interaction. International Journal for Numerical Methods in Engineering, 81(1):48-71, 2010b. 42

T. Rabczuk, G. Zi, S. Bordas, and H. Nguyen-Xuan. A simple and robust threedimensional cracking-particle method without enrichment. Computer Methods in Applied Mechanics and Engineering, 199(37-40):2437-2455, 2010c. 42

Timon Rabczuk, Goangseup Zi, Stephane Bordas, and Hung Nguyen-Xuan. A simple and robust three-dimensional cracking-particle method without enrichment. Computer Methods in Applied Mechanics and Engineering, 199(37-40): 2437-2455, 2010d. 7

Ahmed Ramadan Suleiman and Moncef L Nehdi. Modeling self-healing of concrete using hybrid genetic algorithm-artificial neural network. Materials, 10 (2):135, 2017. x, 24, 25

H Ranaivomanana and N Benkemoun. Numerical modelling of the healing process induced by carbonation of a single crack in concrete structures: Theoretical formulation and embedded finite element method implementation. Finite Elements in Analysis and Design, 132:42-51, 2017. 24

KT Venkateswara Rao, WO Soboyejo, and RO Ritchie. Ductile-phase toughening and fatigue-crack growth in nb-reinforced molybdenum disilicide intermetallic composites. Metallurgical Transactions A, 23(8):2249-2257, 1992. 65 
Huilong Ren, Xiaoying Zhuang, Yongchang Cai, and Timon Rabczuk. Dualhorizon peridynamics. International Journal for Numerical Methods in Engineering, 108(12):1451-1476, 2016. 7

Esteban Samaniego Alvarado. Contributions to the continuum modelling of strong discontinuities in two-dimensional solids. Universitat Politècnica de Catalunya, 2003. 8

PJ Sánchez, AE Huespe, J Oliver, G Diaz, and VE Sonzogni. A macroscopic damage-plastic constitutive law for modeling quasi-brittle fracture and ductile behavior of concrete. International Journal for Numerical and Analytical Methods in Geomechanics, 36(5):546-573, 2012. 10

B Savija and E Schlangen. Modelling the influence of cracking and healing on modal properties of concrete beams. In ICSHM 2015: Proceedings of the 5th International Conference on Self-Healing Materials, Durham, USA, 22-24 June 2015, 2015. 20

E Schlangen, N Ter Heide, and K Van Breugel. Crack healing of early age cracks in concrete. In Measuring, Monitoring and Modeling Concrete Properties, pages 273-284. Springer, 2006. 18

Stewart A Silling and Richard B Lehoucq. Peridynamic theory of solid mechanics. In Advances in applied mechanics, volume 44, pages 73-168. Elsevier, 2010. 7

Abaqus Version Simulia. Abaqus 6.13 documentation. Dassault systemes, 2013, 2013. x, xiii, 41, 85

Jeong-Hoon Song and Young-Cheol Yoon. Multiscale failure analysis with coarsegrained micro cracks and damage. Theoretical and Applied Fracture Mechanics, 72:100-109, 2014. 7

XT Su, ZJ Yang, and GH Liu. Monte carlo simulation of complex cohesive fracture in random heterogeneous quasi-brittle materials: A 3d study. International Journal of Solids and Structures, 47(17):2336-2345, 2010. 11 
Xudong Sun and Julie Yeomans. Optimization of a ductile-particle-toughened ceramic. Journal of the American Ceramic Society, 79(10):2705-2717, 1996. 65

Amirreza Talaiekhozan, Ali Keyvanfar, Arezo Shafaghat, Ramin Andalib, MZ Abd Majid, Mohamad Ali Fulazzaky, Rosli Mohamad Zin, Chew Tin Lee, Mohd Warid Hussin, Norhaliza Hamzah, et al. A review of self-healing concrete research development. Journal of Environmental Treatment Techniques, 2(1): 1-11, 2014. 17

Hossein Talebi, Mohammad Silani, Stéphane PA Bordas, Pierre Kerfriden, and Timon Rabczuk. A computational library for multiscale modeling of material failure. Computational Mechanics, 53(5):1047-1071, 2014. 7

Hossein Talebi, Mohammad Silani, and Timon Rabczuk. Concurrent multiscale modeling of three dimensional crack and dislocation propagation. Advances in Engineering Software, 80:82-92, 2015. 7

Xiaoya Tao and David V Phillips. A simplified isotropic damage model for concrete under bi-axial stress states. Cement and Concrete Composites, 27(6): 716-726, 2005. 9

JG Teng, WC Zhu, and CA Tang. Mesomechanical model for concrete. part ii: applications. Magazine of Concrete Research, 56(6):331-345, 2004. 12

Daniel Trias, Josep Costa, Bodo Fiedler, Thomas Hobbiebrunken, and Jorge E Hurtado. A two-scale method for matrix cracking probability in fibre-reinforced composites based on a statistical representative volume element. Composites science and technology, 66(11):1766-1777, 2006. ix, 12, 13

Kim Van Tittelboom and Nele De Belie. Self-healing in cementitious materialsa review. Materials, 6(6):2182-2217, 2013. 1, 17, 26

Kim Van Tittelboom, Kehinde Adesanya, Peter Dubruel, P Van Puyvelde, and Nele De Belie. Methyl methacrylate as a healing agent for self-healing cementitious materials. Smart Materials and structures, 20(12):125016, 2011. 37, 45 
George Z Voyiadjis and Peter I Kattan. A comparative study of damage variables in continuum damage mechanics. International Journal of Damage Mechanics, 18(4):315-340, 2009. 9

George Z Voyiadjis, Ziad N Taqieddin, and Peter I Kattan. Anisotropic damageplasticity model for concrete. International Journal of Plasticity, 24(10):19461965, 2008. 10

JY Wang, H Soens, Willy Verstraete, and Nele De Belie. Self-healing concrete by use of microencapsulated bacterial spores. Cement and Concrete Research, 56:139-152, 2014. 55, 99

XF Wang, ZJ Yang, JR Yates, AP Jivkov, and Ch Zhang. Monte carlo simulations of mesoscale fracture modelling of concrete with random aggregates and pores. Construction and Building Materials, 75:35-45, 2015a. 12

Xianfeng Wang, Feng Xing, Ming Zhang, Ningxu Han, and Zhiwei Qian. Experimental study on cementitious composites embedded with organic microcapsules. Materials, 6(9):4064-4081, 2013. 46, 60, 87, 88

Xiaofeng Wang and Andrey P Jivkov. Combined numerical-statistical analyses of damage and failure of $2 \mathrm{~d}$ and $3 \mathrm{~d}$ mesoscale heterogeneous concrete. Mathematical Problems in Engineering, 2015, 2015. 12, 46, 87, 88

Xiaofeng Wang, Zhenjun Yang, and Andrey P Jivkov. Monte carlo simulations of mesoscale fracture of concrete with random aggregates and pores: a size effect study. Construction and Building Materials, 80:262-272, 2015b. 12

Xiaofeng Wang, Mingzhong Zhang, and Andrey P Jivkov. Computational technology for analysis of $3 \mathrm{~d}$ meso-structure effects on damage and failure of concrete. International Journal of Solids and Structures, 80:310-333, 2016. 12

Garth N Wells and LJ Sluys. A new method for modelling cohesive cracks using finite elements. International Journal for numerical methods in engineering, 50(12):2667-2682, 2001. 8 
Scott R White, NR Sottos, PH Geubelle, JS Moore, M_R Kessler, SR Sriram, EN Brown, and S Viswanathan. Autonomic healing of polymer composites. Nature, 409(6822):794-797, 2001. x, 2, 35

Peter Wriggers and SO Moftah. Mesoscale models for concrete: Homogenisation and damage behaviour. Finite elements in analysis and design, 42(7):623-636, 2006. 81

Min Wu, Björn Johannesson, and Mette Geiker. A review: Self-healing in cementitious materials and engineered cementitious composite as a self-healing material. Construction and Building Materials, 28(1):571-583, 2012. 17

X Frank Xu and L Graham-Brady. A stochastic computational method for evaluation of global and local behavior of random elastic media. Computer methods in applied mechanics and engineering, 194(42):4362-4385, 2005. 14

$\mathrm{X}-\mathrm{P} \mathrm{Xu}$ and A Needleman. Numerical simulations of dynamic crack growth along an interface. International Journal of Fracture, 74(4):289-324, 1996. 8

JM Yang and SM Jeng. Interface and mechanical behavior of mosi 2-based composites. Journal of Materials Research, 6(3):505-513, 1991. 65

Zhenjun Yang and X Frank Xu. A heterogeneous cohesive model for quasi-brittle materials considering spatially varying random fracture properties. Computer methods in applied mechanics and engineering, 197(45):4027-4039, 2008. ix, 14,16

ZJ Yang, XT Su, Jian Fei Chen, and GH Liu. Monte carlo simulation of complex cohesive fracture in random heterogeneous quasi-brittle materials. International Journal of Solids and Structures, 46(17):3222-3234, 2009. 11

Young-Cheol Yoon and Jeong-Hoon Song. Extended particle difference method for moving boundary problems. Computational Mechanics, 54(3):723-743, 2014a. 7

Young-Cheol Yoon and Jeong-Hoon Song. Extended particle difference method for weak and strong discontinuity problems: part i. derivation of the extended 
particle derivative approximation for the representation of weak and strong discontinuities. Computational Mechanics, 53(6):1087-1103, 2014b. 7

Young-Cheol Yoon and Jeong-Hoon Song. Extended particle difference method for weak and strong discontinuity problems: part ii. formulations and applications for various interfacial singularity problems. Computational Mechanics, 53(6):1105-1128, 2014c. 7

Young-Jun You, Jang-Ho Jay Kim, Ki-Tae Park, Dong-Woo Seo, and Tae-Hee Lee. Modification of rule of mixtures for tensile strength estimation of circular gfrp rebars. Polymers, 9(12):682, 2017. 100

Serguey V Zemskov, Henk M Jonkers, and Fred J Vermolen. Two analytical models for the probability characteristics of a crack hitting encapsulated particles: Application to self-healing materials. Computational materials science, 50(12): 3323-3333, 2011. 2

Serguey V Zemskov, Henk M Jonkers, and Fred J Vermolen. A mathematical model for bacterial self-healing of cracks in concrete. Journal of Intelligent Material Systems and Structures, 25(1):4-12, 2014. 12, 26

Yiming Zhang and Xiaoying Zhuang. A softening-healing law for self-healing quasi-brittle materials: analyzing with strong discontinuity embedded approach. Engineering Fracture Mechanics, 192:290-306, 2018. 24

Shuai Zhou, Hehua Zhu, Zhiguo Yan, J Woody Ju, and Lianyang Zhang. A micromechanical study of the breakage mechanism of microcapsules in concrete using pfc2d. Construction and Building Materials, 115:452-463, 2016. 32

Shuai Zhou, Hehua Zhu, J Woody Ju, Zhiguo Yan, and Qing Chen. Modeling microcapsule-enabled self-healing cementitious composite materials using discrete element method. International Journal of Damage Mechanics, 26(2): 340-357, 2017. x, 32, 33

XQ Zhou and Hong Hao. Mesoscale modelling of concrete tensile failure mechanism at high strain rates. Computers \& Structures, 86(21):2013-2026, 2008. 12 
Hehua Zhu, Shuai Zhou, Zhiguo Yan, Woody Ju, and Qing Chen. A 3d analytical model for the probabilistic characteristics of self-healing model for concrete using spherical microcapsule. Computers and Concrete, 15(1):37-54, 2015. 12, 29 

